\title{
ENVIRONMENTAL FACTORS REGULATE THE FATTY ACID CONTENT OF PRIMARY PRODUCERS AND CONSUMERS IN A FRESHWATER POND IN A WARMING WORLD
}

\author{
by \\ Tarn Preet Parmar \\ BSc (Honours), York University, 2015 \\ A thesis presented to Ryerson University \\ in partial fulfillment of the requirements for the degree of \\ Master of Science \\ in the program of \\ Molecular Science
}

Toronto, Ontario, Canada, 2018

(C) Tarn Preet Parmar, 2018 


\section{AUTHOR'S DECLARATION FOR ELECTRONIC SUBMISSION OF A THESIS}

I hereby declare that I am the sole author of this thesis. This is a true copy of the thesis, including any required final revisions, as accepted by my examiners.

I authorize Ryerson University to lend this thesis to other institutions or individuals for the purpose of scholarly research

I further authorize Ryerson University to reproduce this thesis by photocopying or by other means, in total or in part, at the request of other institutions or individuals for the purpose of scholarly research.

I understand that my thesis may be made electronically available to the public. 


\begin{abstract}
ENVIRONMENTAL FACTORS REGULATE THE FATTY ACID CONTENT OF PRIMARY PRODUCERS AND CONSUMERS IN A FRESHWATER POND IN A WARMING WORLD
\end{abstract}

Tarn Preet Parmar, Master of Science, 2018

Molecular Science, Ryerson University

Examining the impact of abiotic factors on the fatty acid profile of freshwater organisms is an important step in predicting the potential effects of climate change. I designed and deployed a heating system to manipulate the water temperature of a large mesocosm $(>300,000 \mathrm{~L})$ within a pond to simulate future predictions. Heating was observed near the piping system but not throughout the water column. In a separate experiment, the seasonal and depth-related variance in biomass and polyunsaturated fatty acids (PUFA) content of aquatic biofilm, grown on artificial substrates (unglazed ceramic tiles) was examined. Although summer biofilm typically had higher biomass and higher PUFA yield, fall biofilm typically contained higher PUFA content (standardized for biomass). Similarly, deeper biofilm accumulated higher organic matter but contained lower standardized PUFA content. These results suggest that abiotic factors may play a key role in determining the availability of nutritionally-important fatty acids in freshwater ecosystems. 


\section{Acknowledgements}

First, I would like to express my sincere gratitude to Dr. Michael Arts for giving me the opportunity and the memorable experiences during my journey as a graduate student. Thank you for your guidance and support through the many setbacks and encouragement with the triumphs during such an ambitious thesis. The knowledge I have gained under your supervision will be a guiding light in my scientific career.

Second, I want to extend my utmost appreciation to Peter and Elaine Leigh (and Jake) for allowing a bunch of strangers into your backyard and home all in the name of education. This thesis would not have been possible without your kindness and compassion, which also made your backyard an amazing "field station". I hope the extended exposure to research has increased your scientific knowledge, at least about phytoplankton! A special thank you to Dr. Dominik Martin-Creuzburg, who travelled from Germany and was a tremendous help during the climate warming experiment. Despite the outcome, your efforts to the project are greatly appreciated and I look forward to working with you again in Germany!

Field work is demanding but it would have been impossible without the help of numerous field assistants! Thank you to Ledja Rasul and Justin Singh for your sweat and blood during the field season. Special thanks to Shalinda Senanayake and Sydney Wilkinson for providing essential relief as on-call field assistants, especially in colder temperatures. In addition, my thanks extend to Serena Sbrizzi, Bruce Laurich, and Dino Milotic for their help in the field. My sincere appreciation to Dr. Andrew Laursen, Shawn McFadden, and Robert Denning for their willingness to literally dive in the project.

The generosity and support of many have made the work done in this thesis easier and rigorous. Thank you to Drs. Craig Hebert and John Chételat for their interest in the project and 
supplying a generous number of temperature loggers. Special thank you goes to Kelly Bowen for lending her zooplankton nets and filtering apparatus; the latter saving countless hours of vacuum filtering. My sincere appreciation extends to Dr. Claire Oswald for graciously lending her YSI sonde throughout the biofilm experiment. Special thank you extend to Dr. Martha Patricia Celis (and the OMECC) for generously culturing Daphnia and analyzing nutrients. My sincere thanks to Drs. Gideon Wolfaardt and Otini Kroukamp for sharing their knowledge on biofilm and to Evan and Patrick Ronan for their tremendous help with CLSM analysis. I would also like to thank my committee members, Drs. Janet Koprivnikar and Stephanie Melles, for their greatlyappreciated feedback and encouragement throughout the entirety of my degree.

Thank you to all the colleagues, staff, graduate students, and fellow lab mates at Ryerson University. A special thanks to Dr. Ursula Strandberg for being a friend since day 1 at Ryerson. To Kruti Shukla, I simply could not have done this thesis without your help. Like a plant or a biofilm community, you are versatile and adaptive; thank you for all your help with fieldwork (i.e., pond work), experimental design, statistics, writing, presentations, and all the other day-today struggles that are part of being a graduate student. I am forever grateful for your support and encouragement day in and day out.

Finally - thanks to my family for being so supportive throughout my life but especially during my masters. Your unconditional love and support has always been evident but even I did not expect you all to set aside your own responsibilities to help me, especially during the fall biofilm harvesting - a real family experience! Special thanks to my sister for giving up several of her days off to help with light measurements in exchange for conversation and a paid breakfast! Simply put, you are the best mother, father, and sister a field ecologist could ever ask for and I hope I have made you proud. 


\section{Table of Contents}

Preface

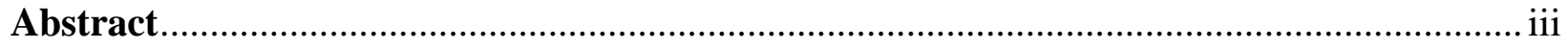

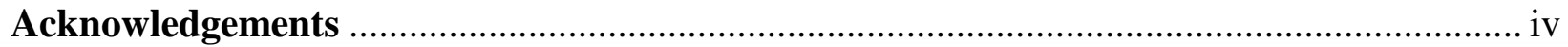

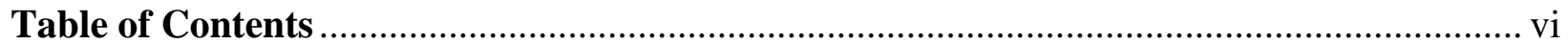

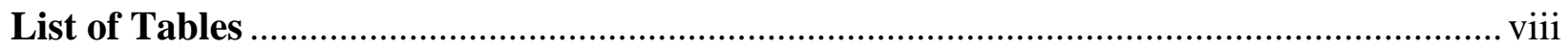

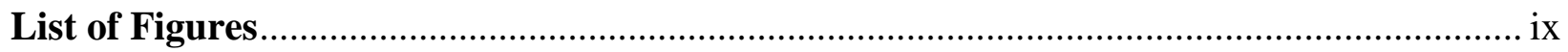

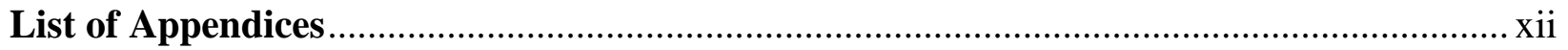

Chapter 1 - Aquatic Biofilms and the Abiotic Factors that Affect Quantity and Nutritional Quality in Freshwater Ecosystems

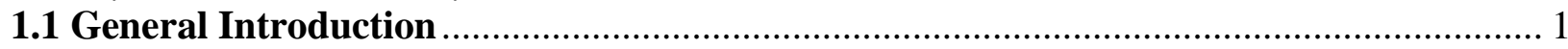

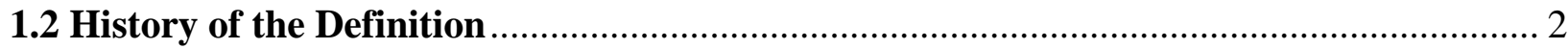

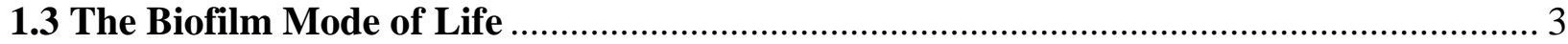

1.3.1 Biofilms in Aquatic Ecosystems ............................................................................ 4

1.3.2 Formation of Aquatic Biofilm on Submerged Surfaces................................................... 4

1.3.3 Types of Interaction Within Aquatic Biofilms .............................................................. 6

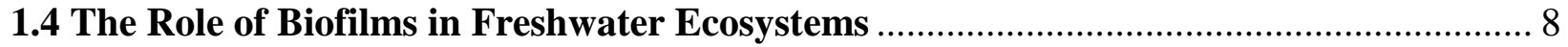

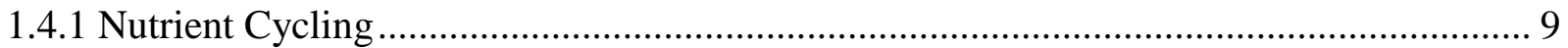

1.4.2 Regulation of Submerged Vegetation .......................................................................... 10

1.4.3 Energy Input to Food Webs ................................................................................... 11

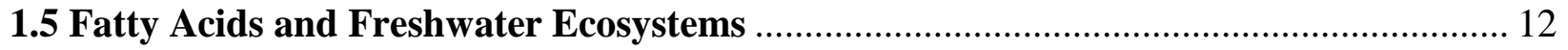

1.5.1 Fatty Acids Production in Phytoplankton ……………............................................... 13

1.5.2 Essential Fatty Acids in Freshwater Pelagic Food Webs ................................................ 14

1.5.3 Essential Fatty Acid Production and Transfer in Freshwater Biofilm ............................ 16

1.6 The Influence of Environmental Factors on Quantity and Nutritional Quality of

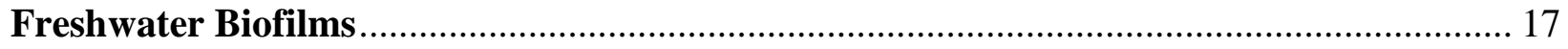

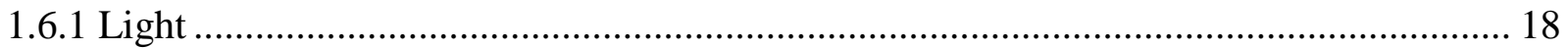

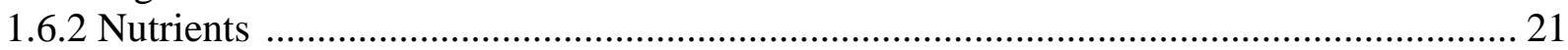

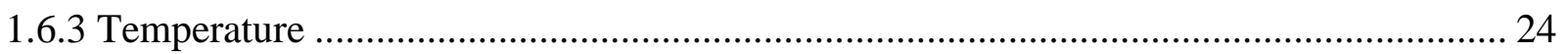

1.7 Climate Change

1.7.1 Freshwater Ecosystems and Climate Change............................................................... 29

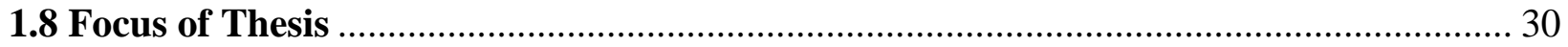

Chapter 2 - Measuring the Effects of Increased Water Temperature on the Production, Transfer, and Re-distribution of Essential Fatty Acids in Freshwater Wetlands

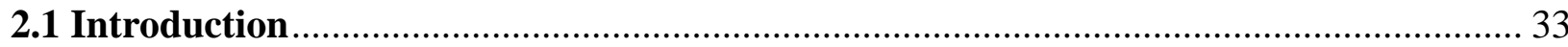

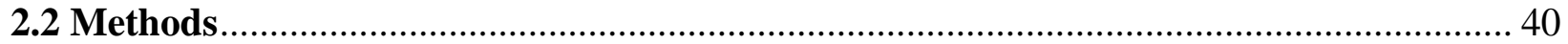

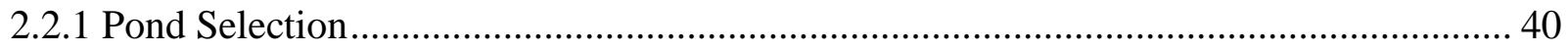

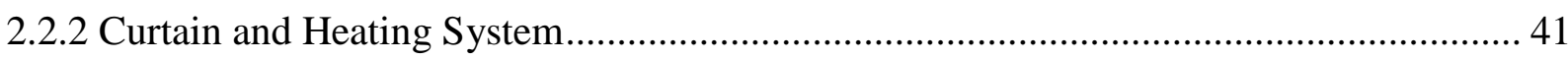

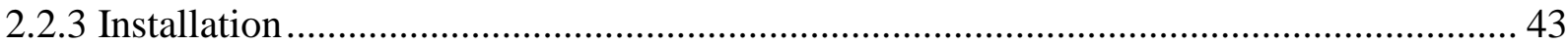

2.2.4 Start of Experiment and Temperature Measurements..................................................... 44

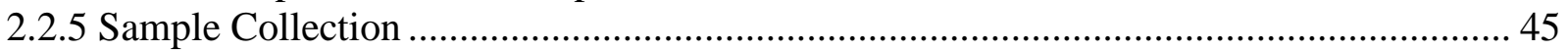




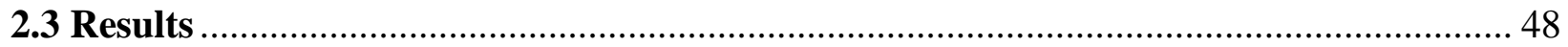

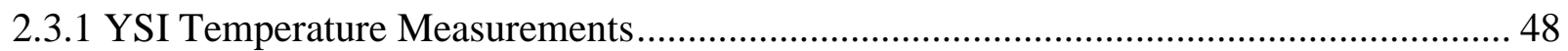

2.3.2 Vertical Thermistor Chain Temperature Measurements ............................................... 49

2.3.3 Horizontal Thermistor Chain Temperature Measurements.............................................. 54

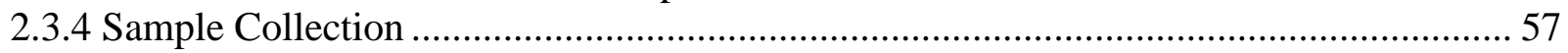

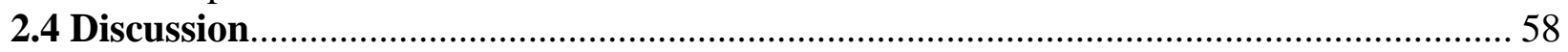

Chapter 3 - Seasonality and Depth Influences the Quantity and the Nutritional Quality of Aquatic Biofilm

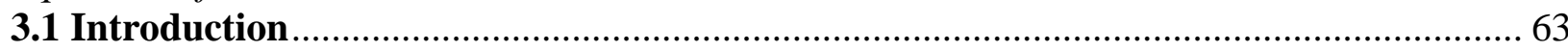

3.2 Methods

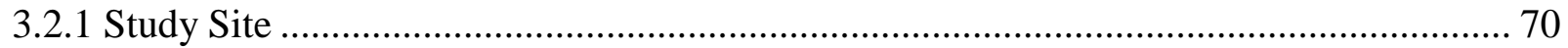

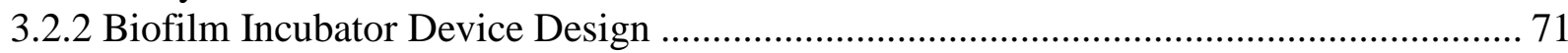

3.2.3 Abiotic Measurements............................................................................................. 72

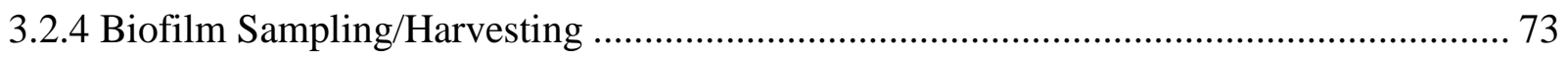

3.2.5 Confocal Laser Scanning Microscopy Analysis .............................................................. 74

3.2.6 Lipid and Fatty Acid Analysis ................................................................................ 75

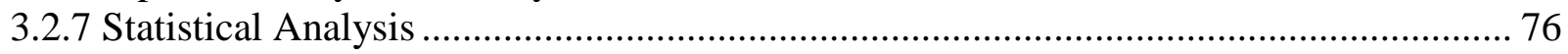

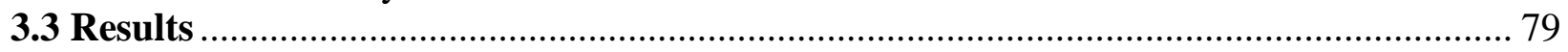

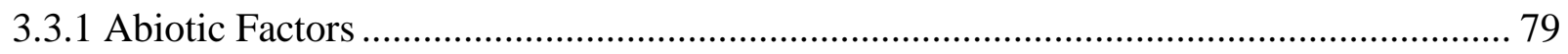

3.3.2 Confocal Laser Scanning Microscopy Analysis ............................................................. 82

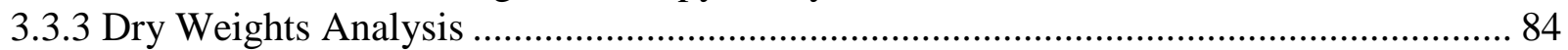

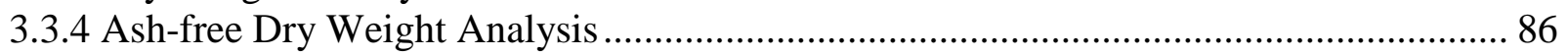

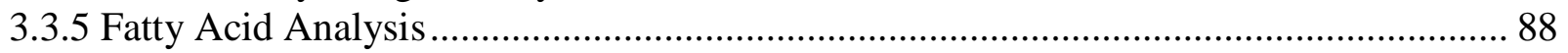

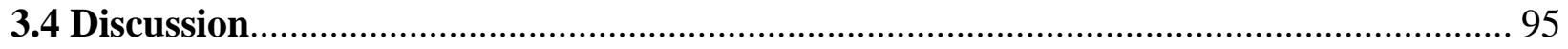

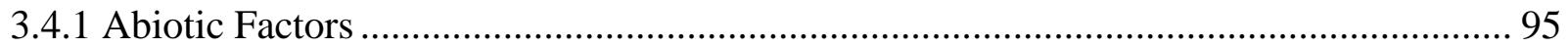

3.4.2 Seasonal, Depth-related, and Spatial Differences in Biofilm Biomass ............................. 96

3.4.3 Seasonal and Depth-related Differences in Biofilm Nutritional Quality ........................ 99

3.4.4 Seasonal and Depth-related Differences in Biofilm Fatty Acid Profile.......................... 102

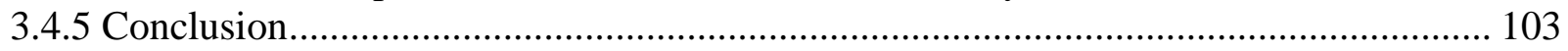

Chapter 4 - Summary and Research Recommendations

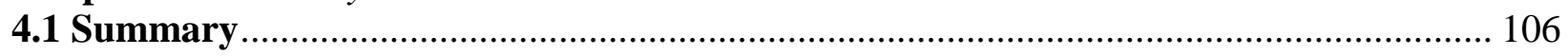

4.2 Research Recommendations ................................................................................ 109

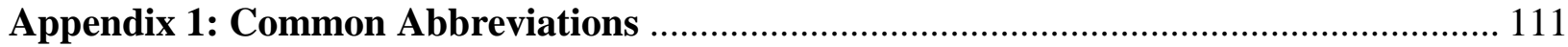

Appendix 2: Chemical Composition of Hard COMBO Media........................................... 112

Appendix 3: Nutrient Concentration between the two Mesocosms (Control and

Experimental) and the Ambient Section ........................................................................ 113

Appendix 4: Identifiable Fatty Acids analyzed ………................................................... 114

Appendix 5: Complete Statistical Summaries Conducted in Chapter 3............................ 115

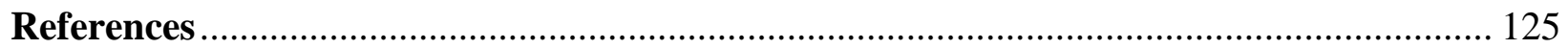




\section{List of Tables}

Table 2.1: Water temperature data $\left({ }^{\circ} \mathrm{C}\right)$ of vertical thermistor chains of all sections in all locations at all depths measured. Values are presented as mean $\pm \mathrm{SD}(n) \ldots \ldots \ldots \ldots \ldots \ldots \ldots \ldots . \ldots \ldots$

Table 2.2: Water temperature data $\left({ }^{\circ} \mathrm{C}\right)$ of vertical thermistor chains of all sections in all locations at all depths measured. Values are presented as mean $\pm \mathrm{SD}(n)$

Table 3.1: Average values for fatty acid responses from biofilm collected in summer and fall. Values are presented as mean $\pm \mathrm{SD}$. Absolute $\mathrm{t}$-values and $\mathrm{p}$-values were determined using a student's t-test after data were separated by season (to remove bimodal distribution). Parameters marked with $\mathrm{a}^{*}$ and $\dagger$ represent response variables that were significant and marginally significant, respectively, for the Hartigans' dip test for unimodality/multimodality (suggesting data were not unimodal). Bolded $\mathrm{p}$-values represent significance...............................88

Table 3.2: Average values for the overall yield $\left(\mathrm{mg} / \mathrm{m}^{2}\right)$ of nutritional fatty acids from biofilm at various depths collected in summer and fall. Values are presented as mean \pm SD. The difference between seasons was determined by a Student's t-test and a * represents significant difference between seasons. Pairwise comparisons (Tukey HSD post-hoc test) were done on each response separately after data were separated by season (to remove bimodal distribution). Groups marked with different letters are significantly different between depths within a single response variable

Table 3.3: Average values for fatty acid responses from biofilm incubated at several depths in summer and fall. Values are presented as mean \pm SD. Pairwise comparisons (Tukey HSD posthoc test) were done on each response separately after data were separated by season (to remove bimodal distribution). Groups marked with different letters are significantly different between depths within a single response variable. All responses are expressed as $\mu \mathrm{g} / \mathrm{mg}$ AFDW, SFA\%, MUFA\%, and MUFA\% which are expressed as the percentage of $\sum F A$ and n3:n6, which is

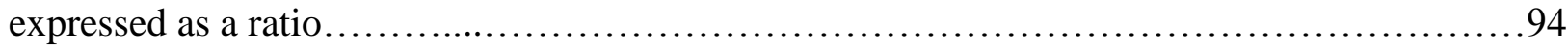




\section{List of Figures}

Figure 1.1: A schematic representation of a mixed aquatic biofilm on an unspecified growth surface. The presence and/or abundance of each taxonomic group can depend on the substrate, environmental, and several abiotic and biotic factors. EPS: extracellular polymeric substances.

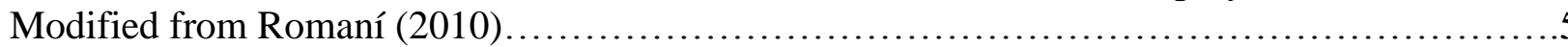

Figure 1.2: Schematic representation of homeoviscous adaptation to different temperatures. Final proportion of saturated (two straight fatty acids) and unsaturated (one straight and one kinked fatty acid) represent the changes in fatty acid proportions due to higher and lower temperatures. Unsaturated fatty acids are substituted in at low temperatures to prevent rigidification while saturated fatty acids at higher temperatures prevent fluidization of membranes

Figure 2.1: Search area for our candidate pond in Greater Toronto Area. The red line highlights the boundaries of the search area, $2,500 \mathrm{~km}^{2}$. Image captured on Google Maps.

Figure 2.2: Schematic design of experimental setup. The curtain (blue lines) divided the section into control (top), experimental (bottom), and ambient (right). Pool heater and pump were installed near the pond (black box with lightning bolt) connected to a radiator-style heating system composed of alternating PVC (white lines) and aluminum (black lines)

pipes.

Figure 2.3: Location of temperature probes during experiment. Vertical thermistor chains were deployed in three locations: corner (green box), middle (red box), and shallow (yellow box) to represent different depths. Black lines represent the horizontal thermistor chains that were deployed along the sediment in east (right) and west (left) locations

Figure 2.4: Design of floating insect emergence traps. All the components of the traps (A) are constructed to a (B) fully-built aquatic insect emergence trap. Traps were built using the design suggested by Cadmus et al. (2016)..............................................46

Figure 2.5: Temperature measurements (via YSI) of three pond sections on June 11th at varying depths. All measurements were taken within an hour of noon in the middle of each section.

Figure 2.6: Temperature measurements (via YSI) of three pond sections on June 20th at varying depths. All measurements were taken within an hour of noon in the middle of each section.

Figure 2.7: Temperature difference between control (black line) and experimental (grey line) vertical thermistor chains (corner spot). Subpanels represent different depths; (A) 0.5 meters from bottom, (B) 1.0 meters from bottom, (C) 1.5 meters from bottom, (D) 2.0 meters from bottom, and (E) 0.25 meters from top. 
Figure 2.8: Temperature difference between control (black line), ambient (dark grey line), and experimental (light grey line) vertical thermistor chains (middle spot). Subpanels represent different depths; (A) 0.5 meters from bottom, (B) 1.0 meters from bottom, (C) 1.5 meters from

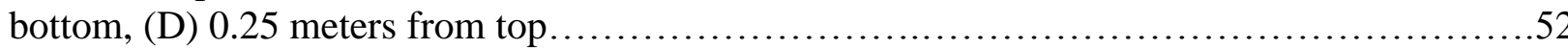

Figure 2.9: Temperature difference between control (black line) and experimental (grey line) vertical thermistor chains (shallow spot). Subpanels represent different depths; (A) $0.5 \mathrm{~m}$ from

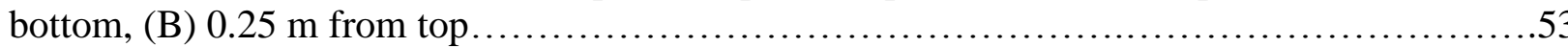

Figure 2.10: Temperature difference between control (black line) and experimental (grey line) horizontal thermistor chains (West location). Subpanels represent different distance from curtain;

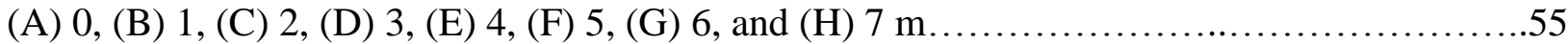

Figure 2.11: Temperature difference between control (black line) and experimental (grey line) horizontal thermistor chains (East location). Subpanels represent different distance from curtain;

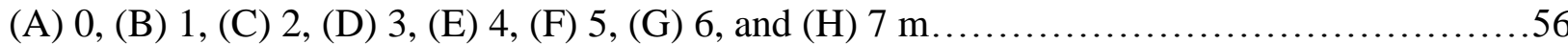

Figure 3.1: Aerial photograph of pond. Incubator platforms are deployed in a randomized block design along three transects (from left to right: Block A, B, and C). Photo taken with DJI Phantom 4 drone on December $4^{\text {th }}, 2017$ ( 1 PM). Photo credits: George Torrance

Figure 3.2: Unglazed ceramic tiles incubated on biofilm incubator devices deployed. Incubators were joined together for the picture but were moved into final position $\sim 1$ meter apart.

Figure 3.3: Seasonality of depth-effect differences in water temperature $\left({ }^{\circ} \mathrm{C}\right.$ ) in summer (grey fill - left y-axis) and fall (white fill - right y-axis). Groups marked with different letters are significantly different (Tukey HSD post-hoc test using the season $\times$ depth interaction term). No differences were observed between depths in fall, therefore, the letter ("A") above the bars was removed for clarity purposes

Figure 3.4: Seasonality of depth-related difference in PAR irradiance $\left(\mathrm{mW} / \mathrm{cm}^{2}\right)$ in summer (grey fill) and fall (white fill). Groups marked with different letters are significantly different (Tukey HSD post-hoc test using the season $\times$ depth interaction term).

Figure 3.5: Seasonality of depth-related difference in (A) UV-R, (B) UV-A, and (C) UV-B irradiance $\left(\mathrm{mW} / \mathrm{cm}^{2}\right)$ in summer (grey fill) and fall (white fill). Groups marked with different letters are significantly different (Tukey HSD post-hoc test of the season $\times$ depth interaction)

Figure 3.6: The effect of depth on biofilm bio-volume $\left(\mu \mathrm{m}^{3} / \mu \mathrm{m}^{2}\right)$ in summer and fall in summer (grey fill) and fall (white fill). Groups marked with different letters are significantly different (Tukey HSD post-hoc test using the season $\times$ depth interaction term). 
Figure 3.7: Seasonal and depth-related effects on the mean thickness $(\mu \mathrm{m})$ of biofilm in summer (grey fill) and fall (white fill). Groups marked with different letters are significantly different (Tukey HSD post-hoc test using the season $\times$ depth interaction term).

Figure 3.8: Seasonal effect of spatial variability in biofilm mean thickness $(\mu \mathrm{m})$ in summer (grey fill) and fall (white fill). Groups marked with different letters are significantly different (Tukey

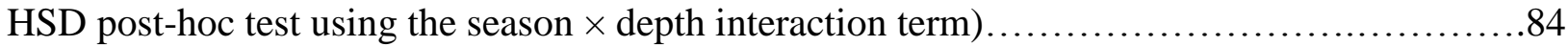

Figure 3.9: Seasonality of depth-related effect on biofilm dry weight $\left(\mathrm{mg} / \mathrm{m}^{2}\right)$ in summer (grey fill) and fall (white fill). Groups marked with different letters are significantly different (Tukey HSD post-hoc test using the season $\times$ depth interaction term $) \ldots \ldots \ldots \ldots \ldots \ldots \ldots \ldots \ldots \ldots \ldots \ldots$

Figure 3.10: The spatial variability of biofilm dry weight $\left(\mathrm{mg} / \mathrm{m}^{2}\right)$ in summer (grey fill) and fall (white fill). Groups marked with different letters are significantly different (Tukey HSD post-hoc

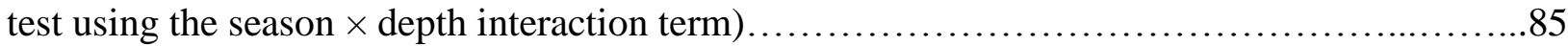

Figure 3.11. Seasonality of depth-related effect on biofilm ash-free dry weight $\left(\mathrm{mg} / \mathrm{m}^{2}\right)$ in summer (grey fill) and fall (white fill). Groups marked with different letters are significantly different (Tukey HSD post-hoc test using the season $\times$ depth interaction term)....

Figure 3.12. Seasonality of depth-related effect on the percentage organic matter of biofilm collected in summer (grey fill) and fall (white fill). Groups marked with different letters are significantly different (Tukey HSD post-hoc test using the season $\times$ depth interaction term).

Figure 3.13. Seasonality of block-related effect on the percentage organic matter of biofilm collected in summer (grey fill) and fall (white fill). Groups marked with different letters are significantly different (Tukey HSD post-hoc test using the season $\times$ depth interaction term).

Figure 3.14: Nutritional quality of biofilm incubated at depths in (A) summer and (B) fall determined by $\sum$ n-3 PUFA (dark grey fill), EPA + DHA (stripped light grey fill), and $\sum$ n-6 PUFA (white fill) content. Depths marked with different letters represent groups that are significantly different within one response (Tukey HSD post-hoc test): $\sum$ n-3 PUFA (bolded upper case), EPA + DHA (bolded lower case), and $\sum$ n-6 PUFA (italicized upper case)..........91

Figure 3.15: FA biomarker content in summer (grey fill) and fall (white fill) biofilm incubated at several depths. The FA (A) 16:1n-7 is a diatom biomarker and (B) $\sum$ odd chain-length FA are bacterial biomarkers. Groups marked with different letters are significantly different (Tukey HSD post-hoc test). Upper (summer) and lower (fall) cases represent separate post-hoc tests as seasons were analyzed separately....

Figure 3.16: The depth-related effect on the unsaturation index (UI) of biofilm incubated in summer (grey fill) and fall (white fill). Groups marked with different letters are significantly different (Tukey HSD post-hoc test). Upper (summer) and lower (fall) cases represent separate post-hoc tests as seasons were analyzed separately. 


\section{List of Appendices}

Appendix 1: Common Abbreviations ...........................................111

Appendix 2: Chemical Composition of Hard COMBO Media............................112

Appendix 3: Nutrient Concentration between two Mesocosms (Control and Experimental) and the Ambient Section........................................................ 113

Appendix 4: Identifiable Fatty Acids Analyzed.......................................114

Appendix 5: Complete Statistical Summaries Conducted in Chapter 3......................115 


\section{Chapter 1}

\section{Aquatic Biofilms and the Abiotic Factors that Affect Quantity and Nutritional Quality in Freshwater Ecosystems}

\section{$\underline{1.1 \text { General Introduction }}$}

Submerged surfaces, unless treated with an anti-microbial agent, will rapidly be colonized by a wide-range of microorganisms from the surrounding environment. Complex and diverse assemblages of microorganisms colonizing surfaces have been discovered in fossil records dating back at least 3.5 billion years (Rasmussen 2000; Westall et al. 2001; Noffke et al. 2013), demonstrating their antiquity. Attached microbial communities can be found in a wide range of modern day environments including aquatic ecosystems, soil, extreme thermal environments, man-made water pipes, and within the human body (Donlan 2002).

Unattached and planktonic microorganisms can detect and adhere to a given surface, via the secretion of a sticky matrix of sugars and proteins, allowing other microbes to attach and ultimately, leading to an assemblage of cooperating and competing microorganisms (i.e., the biofilm; Flemming \& Wingender 2010). Given their ubiquity, it is not surprising that most people will recognize the brownish or greenish slimy coat (a common characteristic of these communities), which is commonly observed on submerged surfaces such as rocks and boardwalks. Compared to the planktonic mode of life, these embedded microbes are relatively more protected from grazing predators and environmental stressors while also exchanging nutrients, metabolites, and genetic material. However, microorganisms in such close proximity are also competing for external (e.g., light and nutrients) and internal (i.e., gases) resources (Dang \& Lovell 2016). Aquatic scientists acknowledge the importance of these microbial communities in the function and health of an aquatic ecosystem and have studied these assemblages for over 100 years (Seligo 1905; Hentschel 1916).

My literature review introduces aquatic biofilms, their biological significance, structure and member constituents, as well as the abiotic factors that ultimately affect freshwater biofilm quantity and nutritional quality. First, I review the historical background of attached microbial communities to provide a working definition that will be applied for the rest of the thesis. Then, I discuss aquatic biofilms; their general characteristics, the formation process, and interactions between microbial constituents. I also address the role these communities of microorganisms, as 
a collective entity, play within freshwater ecosystems. I then examine how key abiotic factors (light, nutrients, and water temperature) and climate warming, in general, may affect the measurable quantity and nutritional quality of freshwater biofilms. My review concludes with the main thesis hypotheses and objectives and selected aspects of the application of my work to aquatic ecosystem science.

\subsection{History of the Definition}

My literature search revealed that the terminology and definitions used to characterize attached microbial communities on submerged surfaces has been both inconsistent and redundant. Seligo (1905) defined microscopic plants and animals living, attached or otherwise, on plants, stones, logs, specifically, as aufwuchs (German for "surface growth" or “overgrowth"). Hentschel (1916) also introduced bewuchs to connotate the same microbial community, however, the description also extended to larger organisms, such as insect larvae. Willer (1923) suggested a distinction between the two related terms; aufwuchs was to be used for growth on living substrates while bewuchs was to be used to distinguish growth on dead substrates. Despite the clear distinction, aufwuchs seemed to be preferred in the literature, however, it was used to describe complex attached microbial communities growing on any surface, rather than a specific substrate (Cooke 1956). In general, early terminology had a broad criterion for classification of organisms and had the potential to overlap in definition; for example, aufwuchs could include sponges and invertebrates (Ruttner et al. 1953). At the same time aufwuchs was introduced, attached algae cells were being discovered in ponds and streams and described as epiphytic, non-parasitic plant growth on plant material (Fritsch 1906; Brown 1908). Consequently, especially in American literature, the word periphyton, was originally introduced to describe all organisms attached and growing on, specifically, artificiallysubmerged surfaces (Behning 1924) but was later altered to include all submerged surfaces (Behning 1928). The definition of periphyton was later modified to describe a slime-covered community of organisms growing on a submerged surface (Young 1945), excluding macroinvertebrates (Reid 1961), and, finally, to only be applied to algal communities attached to a wide range of surfaces (Round 1984).

Ultimately, by 1983, the definition of periphyton morphed into a broadly-encompassing term that included a complex community of algae, bacteria, fungi, animals, inorganic and 
organic detritus attached to a surface within an organic matrix of polysaccharides and proteins (Wetzel 1983). Wetzel's broad definition of periphyton paralleled the term biofilm, which was initially used to describe a similar community matrix found in wastewater treatment facilities (Mack et al. 1975). The terms periphyton and biofilm have since been used interchangeably, however, periphyton has been typically linked with photosynthetic microorganisms and energy nutrient dynamics in ecosystems (Saikia 2011). Recognizing the complexity of microbial communities, my review will apply biofilm as a blanket term to simply refer to structured and irreversible-attached microbial communities, excluding animals such as the invertebrates that are otherwise free to move in and out of the structured microbial community. Considering this thesis, biofilms, in general, are a community of microorganisms (e.g., bacteria, archaea, algae, fungi, viruses, and protozoa) that are irreversibly (i.e., cannot be easily removed by water flow) associated with a surface and are enclosed in a polymeric matrix. Specificity, whenever possible, will be used when referring to biofilm attached to specific substrate including rocks (epilithic), sandy sediment (epipsammic), muddy sediment (epipelic), and living (epiphytic) and dead (epixylic) plant material (Mora-Gómez et al. 2016).

\subsection{The Biofilm Mode of Life}

Genetically (Mah et al. 2003), phenotypically (Favre et al. 2018), and physiologically (Chao et al. 2010; Dubois-Brissonnet et al. 2016) there are distinct differences between planktonic cells suspended in media (i.e., not attached to a surface) and cells of the same species growing within a biofilm. In general, the biofilm mode of life, compared to a planktonic mode, provides many benefits to the cells within the community including, increased protection from grazers (Matz et al. 2005; Raghupathi et al. 2017), reduced negative impact from environmental stressors (Teitzel \& Parsek 2003; Emtiazi et al. 2004; Choi et al. 2010; Booth et al. 2011), and increased genetic diversity due to high rates of horizontal gene transfer, due to cell proximity, via plasmids (Molin \& Tolker-Nielsen 2003; Madsen et al. 2012; Stalder \& Top 2016). Cooperative resource sharing has been demonstrated between peripheral and interior cells within a biofilm (Liu et al. 2015) and between adjacent biofilm populations ( $2 \mathrm{~mm}$ apart) of the same species in nutrient-limited environments (Liu et al. 2017). Communication (via electrical signaling) within and between biofilm populations allows growth rates to synchronize in an antiphase oscillation pattern, allowing for maximum growth of both populations (Liu et al. 2017). 
On the contrary, the close proximity of microorganisms within a biofilm can result in increased exploitative and/or interference competition (Rendueles \& Ghigo 2015). Indirectly, certain species can outcompete other microbes for essential nutrients, such as carbon, phosphorus, and iron (Bradshaw et al. 2002; Weaver \& Kolter 2004; Oehmen et al. 2007) or gases, such as oxygen (Yamamoto et al. 2010). Microorganisms within a biofilm can also directly compete with other microbes by producing anti-microbial compounds (Yan et al. 2003; Rendueles et al. 2014), which inhibit communication (Augustine et al. 2010) and adhesion (Kim \& Kim 2009; Rendueles et al. 2011) of competing species. Despite the potential of competition, diverse and complex multi-species biofilms can be found within a wide range of ecosystems, including in aquatic ecosystems, highlighting the benefits of living within a biofilm.

\subsubsection{Biofilms in Aquatic Ecosystems}

Biofilms can be found on a wide range of substrates in all types of aquatic ecosystems including, freshwater (e.g., shallow wetlands, rivers, streams, lakes and reservoirs), marine (e.g., intertidal, subtidal and deep-sea zones), transitional (e.g., estuaries and bays), and groundwater systems (e.g., aquifers and subterranean springs). Early studies on aquatic biofilms deployed standard glass slides in various spatial configurations (e.g., vertically or horizontally) to grow microorganisms in an effort to reduce the variability associated with biofilm growing on natural substrates (Hentschel 1916). Combined with the advent and growing use of scanning electron microscopy, the composition and development of these film-forming complex microbiological communities was revealed (Mack et al. 1975). Subsequently, it was suggested that the type of surface may influence early cell accumulation and distribution (Terlizzi \& Faimali 2010; Pandey et al. 2014); however, generalizations (see next sections) can be made about the development of microbes into an aquatic biofilm on a new submerged surface.

\subsubsection{Formation of Aquatic Biofilm on Submerged Surfaces}

The wide range of microorganisms found in aquatic ecosystems allows for aquatic biofilms to be composed of a diverse group of constituent members including bacteria, archaea, eukaryotic algae, cyanobacteria, protozoa, fungi, and multicellular microorganisms (Fig. 1.1). The diversity of microorganisms within aquatic biofilm is strongly dependent on the local microbial community, abiotic conditions, biotic interactions, and substrate characteristics 
(Besemer 2015). Any solid surface that is submerged in water is, within seconds, covered in a layer of adsorbed molecules (Baty et al. 1996; Bos et al. 1999), typically consisting of polysaccharides, proteins, and glycoproteins (Compere et al. 2001; Jain \& Bhosle 2009). These molecules form a conditioning film prior to microbial colonization and their composition and abundance can influence colonization dynamics (Dang \& Lovell 2000; Palmer et al. 2007; Andrews et al. 2010; Camps et al. 2014). Microbial adhesion occurs immediately after surface conditioning and typically follows a two-step process: 1) initial reversible adhesion that can be disrupted with minimal stress, followed by, 2) irreversible adhesion (Characklis \& Cooksey 1983). The first reversible adhesion step requires bacterial cells, primarily, to excrete a sticky matrix of extracellular polymeric substances (EPS; refer to Appendix 1 for a list of abbreviations), which then conditions the surface for other microorganisms to attach (Mack et al. 1975); an essential step in biofilm community formation ( $\mathrm{Vu}$ et al. 2009). This primary step is mediated by weak interaction forces (e.g., van der Waals) and flow-generated shear stress can readily break these weak forces and disrupt initial adhesion, thereby preventing permanent adhesion (Thomen et al. 2017). Therefore, water flow (i.e., shear stress) is a key factor in both steps of adhesion because, although it exposes and mobilizes the accumulation of initial bacterial cells and facilitates the continued colonization by microorganisms (Abe et al. 2000), it can also prevent permanent adhesion.

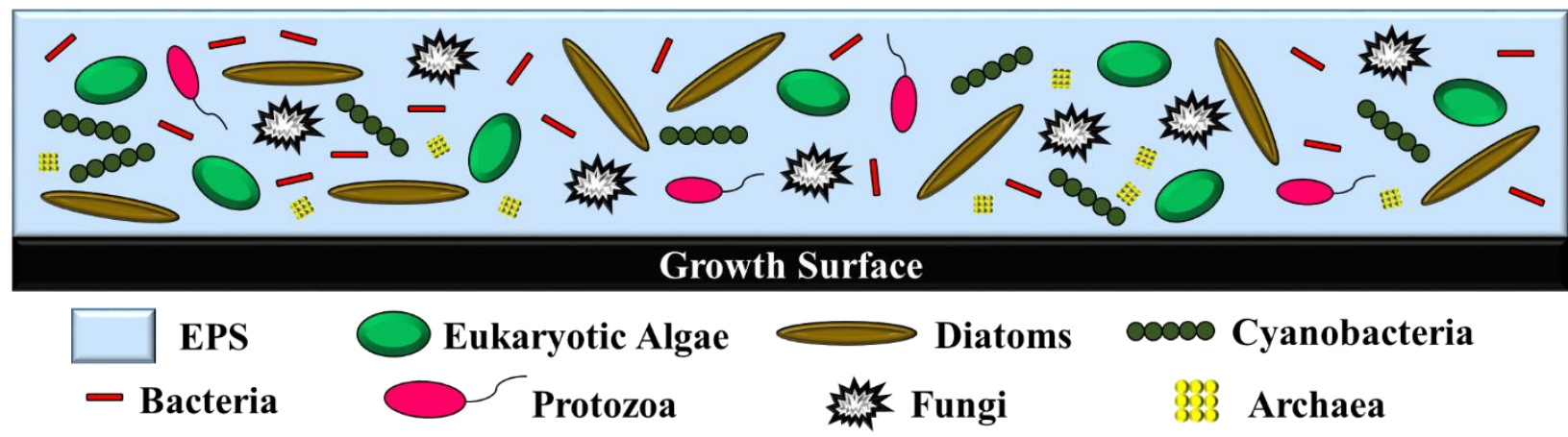

Figure 1.1: A schematic representation of a mixed aquatic biofilm on an unspecified growth surface. The presence and/or abundance of each taxonomic group can depend on the substrate, environmental, and several abiotic and biotic factors. EPS: extracellular polymeric substances. Modified from Romaní (2010).

Following the initial establishment period, a wide range of microorganisms will continue to adhere and release EPS, which continues the process towards producing a mature irreversible biofilm (Barranguet et al. 2004). The type of ecosystem (Oemke \& Burton 1986; Sekar et al. 
2004), microorganisms present (Roeder 1977), and interacting abiotic factors (Horner \& Welch 1981; Fairchild \& Everett 1988; Bothwell et al. 1993) influence the course of succession and the final composition of a mature biofilm. As the biofilm community grows, cells continuously detach through shearing and sloughing (continuous removal of small portions and random massive removal of biofilm, respectively) via continuous water flow or abrupt disturbances (Characklis \& Cooksey 1983). Although both cell recruitment and detachment occur in a biofilm, the EPS matrix is the main component that maintains biofilm composition.

The polymeric matrix, composed of EPS as discussed earlier, is crucial in the formation and maintenance of biofilm as it mediates adhesion to surfaces and other cells and provides mechanical integrity and stability. Furthermore, its complex structure offers protection from grazers and environmental stressors, serves as a nutrient and energy-rich store, and forms the foundation for a cohesive network for cell-to-cell communication (Wotton 2004). As with the term biofilm, the terminology and definition of EPS has changed through the years. In general, EPS describes a collection of high-molecular-weight biosynthetic polymers either secreted by microorganisms, products of cellular lysis, or produced via the hydrolysis of macromolecules (Wingender et al. 1999). The EPS is highly hydrated and is 99\% water and would collapse if dehydrated (Cooksey 1992). The organic portion of EPS is composed mostly of polysaccharides but also includes proteins, lipids, extracellular DNA, nucleic acids, humic substances, and membrane vesicles (Decho 1990; Flemming \& Wingender 2010; Salama et al. 2016). Generally, the structure of EPS in biofilm communities includes an interwoven, 3D matrix, of fluid channels that allow transport of nutrients, gases and waste products (de Beer et al. 1994, 1996). Environmental factors can influence EPS production (Wolfstein \& Stal 2002), however, both bacteria and algae can produce EPS during biofilm maturation (Characklis \& Cooksey 1983), which leads to a biofilm community with both cooperative and competitive interactions among microorganisms.

\subsubsection{Types of Interaction within Aquatic Biofilms}

After submerged surfaces have been conditioned, they are rapidly colonized by microorganisms, including bacteria, archaea, fungi, protozoa, eukaryotic algae, and cyanobacteria (Fröls 2013; Flemming et al. 2016). The latter two photoautotrophs are typically only present when submerged surfaces are exposed to light although chemoautotrophs may also, 
where the appropriate metabolic substrates are abundant, be present in the absence of light (e.g., deep-sea vent biofilms; Sievert \& Vetriani 2012). Multi-species biofilms can harbor several types of interactions including a mutualistic relationship between autotrophs and heterotrophs, competition between microbes, and multi-trophic level predation.

A key interaction in aquatic biofilms is the symbiotic relationship between autotrophs (e.g., algae and cyanobacteria) and heterotrophs (e.g., bacteria, archaea, and fungi). In mixed biofilms, autotrophic and heterotrophic microorganisms are often strongly associated (Romaní \& Sabater 1999; Gao et al. 2004; Carr et al. 2005) and create mutualistic relationships, especially in extreme environments such as Antarctica or even Earth's lower orbit (Thomas \& Dieckmann 2002; Cockell et al. 2011)! The presence of bacteria has been shown to encourage the recruitment of algal cells into biofilms, and vice versa, (Hodoki 2005) and, once a stable biofilm has formed, a mutualistic exchange of nutrients between algal and bacterial cells may occur (Haack \& McFeters 1982a; b; Wetzel 1993; Neely \& Wetzel 1995). Algal exudates, mainly carbohydrates (Myklestad 1995), and lysed algal cellular products provide heterotrophic bacteria with an organic carbon source (Coveney \& Wetzel 1989; Wyatt \& Turetsky 2015), which increases bacterial growth within the biofilms (Murray et al. 1986; Espeland et al. 2001). In return, bacterial cells in biofilms can regenerate essential nutrients, both phosphorus and nitrogen, which can then be absorbed by autotrophic algae and cyanobacteria (Rier \& Stevenson 2002; Carr et al. 2005; Sharma et al. 2005). The importance of these recycled nutrients is evident in low-nutrient systems, as algal cells may be more reliant on bacteria-mediated recycled nutrients than nutrients in the water column (Jones \& Lock 1993; Rier \& Stevenson 2001; Scott et al. 2008). Tightly-associated communities of algae and bacteria can reciprocally provide essential gases, especially in thick biofilms where concentrations can reach critically-low levels near the substrate (Sand-Jensen et al. 1985; Carlton \& Wetzel 1987; Jones et al. 2000; Kühl \& Polerecky 2008). Bacterial-produced carbon dioxide $\left(\mathrm{CO}_{2}\right)$ can be incorporated in algal cells resulting in increased algal biomass while algal-produced oxygen can satisfy oxygen requirements of bacterial communities (Tison \& Lingg 1979; Schiefer \& Caldwell 1982). The consumption of oxygen by bacterial cells can also reduce oxygen damage (i.e., reactive oxygen species; ROS) to algal cells embedded within a biofilm (Mouget et al. 1995).

Although nutrient recycling and gas exchange within a biofilm is essential for overall biofilm growth and biomass (Mulholland et al. 1995), competition can occur between 
microorganisms. Microorganisms within an aquatic biofilm can compete for nutrients (Burkholder et al. 1990; Sommer 1996; Mitbavkar \& Anil 2007) or release allelopathic chemicals that either hinder growth or kill other microbes within the community (Juttner \& Wu 2000; Leflaive et al. 2008; Leflaive \& Ten-Hage 2009). Similar to interaction within the pelagic ecosystem, aquatic biofilms can also be an arena for higher trophic level interactions such as protozoa feeding on bacteria and algae (Augspurger et al. 2008; Früh et al. 2011; Risse-Buhl et al. 2012), rotifers feeding on protozoa, bacteria, and detritus (Augspurger et al. 2008; Dopheide et al. 2011; Mialet et al. 2013), and nematodes grazing on algae (Majdi et al. 2011). Clearly there are many types of interactions that can and do occur at cellular levels in an aquatic biofilm, however, consideration must also be given to the concept that the collective community within a biofilm can act as a "single organism" and participate in essential ecosystem-wide processes.

\subsection{The Role of Biofilms in Freshwater Ecosystems}

Aquatic biofilms play an important role in maintaining the health and productivity of freshwater ecosystems. Freshwater biofilms are composed of a diverse community of microbes and therefore, are involved in several ecosystem processes, including primary productivity (Wetzel 1964; Underwood et al. 2005), carbon and nutrient cycling (Paul et al. 1991; Sekar et al. 2002; Dodds 2003; Lyon \& Ziegler 2009), and supporting food webs (Simon et al. 2003). Some of these ecological functions provide crucial ecosystem services to society, for example, wastewater treatment (Nicolella et al. 2000; Ehlers \& Turner 2012), stormwater monitoring and management (Ancion et al. 2014), and bioremediation (Singh et al. 2006; Pastorella et al. 2012). Contaminates (e.g., metals) can accumulate within biofilms at higher concentrations than within water or sediments (Kiffney \& Clements 1993; Besser et al. 2001), which can lead to biomagnification in biofilm grazers (Besser et al. 2001; Farag et al. 2003). This, however, also provides the opportunity to use biofilms as a critical tool for monitoring contamination in natural ecosystems (Leguay et al. 2016). Biofilm attachment and growth can also cause problems for humans, such as biofouling (Flemming 2008), drinking water contamination (Wingender \& Flemming 2011), and microbial-induced corrosion (Videla \& Herrera 2005). The following subsections will focus on three ecosystem processes that are performed by biofilms in freshwater ecosystems: nutrient dynamics, regulation of submerged vegetation, and energy inputs into food webs. 


\subsubsection{Nutrient Cycling}

In freshwater systems, nutrients support entire ecosystems as they promote the growth of the primary producers that form the base of complex food webs. Phosphorus and nitrogen are essential nutrients required for photosynthetic organisms (e.g., eukaryotic algae, cyanobacteria, and macrophytes) in freshwater ecosystems, however, high concentrations of these nutrients can lead to cyanobacterial dominance and subsequent eutrophication (Watson et al. 1997; Downing et al. 2001). Biofilms contain diverse communities of microbes that, collectively, can be a major regulator of nutrient dynamics in freshwater ecosystems. Many studies have shown that pelagic phytoplankton have higher nutrient uptake rates from the water column than biofilm for both nitrogen (Reuter et al. 1986; Reuter \& Axler 1992) and phosphorus (Steinman et al. 1997; Hwang et al. 1998). However, these studies measured uptake rates and kinetic parameters (e.g., half saturation constants $\left[\mathrm{K}_{\mathrm{m}}\right]$ ) in short-term laboratory experiments using isolated communities in bottle incubations. At larger spatial and temporal scales (e.g., mesocosm experiments), biofilm communities can uptake more added nutrients than phytoplankton (Björk-Ramberg 1985; Mazumder et al. 1989; Hansson 1990; Blumenshine et al. 1997). Mesocosm and whole-lake experiments were subsequently conducted; auspiciously to confirm the insignificant role of biofilm water-column nutrient uptake (as compared to uptake by pelagic phytoplankton as seen in small-scale experiments). However, biofilm was found to sequester more than half of the added nutrients (Axler \& Reuter 1996)!

The importance of water-column nutrients to biofilm has been shown in high-nutrient systems where inorganic nutrients (which are preferred by biofilm communities rather than the energetic process of converting the organic forms) are abundant and readily available (Havens et al. 2001b). Cui and colleagues (2017) introduced 200 naturally-colonized hanging "biocontactors" (i.e., 2-meter long structures covered in polyvinylidene chloride fibers to maximize biofilm attachment and growth) into a mesocosm within eutrophic Lake Seo (South Korea) in an effort to investigate the ability of biofilm to uptake nutrients and therefore, control cyanobacteria blooms. The addition of biofilm into the mesocosm was able to successfully prevent the expected cyanobacteria bloom (observed for the previous 5 years), demonstrated by a $94 \%$ reduction in chlorophyll $a$ ( $\operatorname{chl} a$ ) concentration (Cui et al. 2017). In conclusion, biofilm communities can compete for bioavailable nutrients in the water column, thus reducing concentrations of nutrients 
available for pelagic microbes, however, in nutrient-poor systems biofilm communities may rely on a different source of nutrients other than the water column.

Although nutrients in the water-column may be the most bioavailable, it is not the only source of nutrients in freshwater ecosystems. Nutrient concentrations in the pore water between sediment particles can be 5 - 100x higher than water-column concentrations (Schindler et al. 1987; Hagerthey \& Kerfoot 1998; Vadeboncoeur \& Lodge 2000). Epipelon (i.e., biofilm growing on sediment) can use nutrients in the sediment for growth, sequentially reducing the resulting concentration in the water column (Hansson 1988, 1989). This happens because, during summer months, anoxic conditions at the sediment-water interface stimulates the release of inorganic nutrients into the water column (Golterman 1995). In clear low-nutrient or shallow systems where light can penetrate to the bottom, epipelic algae can super-saturate the sediment surface with oxygen, immobilizing the release of phosphorus to the overlying water (Carlton and Wetzel 1988; Woodruff et al. 1999). This can be seen at short time scales as diurnal decreases in light have led to accelerated nutrient release from the sediment (Carlton \& Wetzel 1988).

Biofilm-colonized surfaces embedded into the sediment have been shown to prevent cyanobacterial blooms by preventing phosphorus from leaching into the water column (Wu et al. 2010). Biofilm communities, whether directly (i.e., higher uptake rates) or indirectly (i.e., sequester sediment nutrients), play a large role in regulating the nutrient concentration within freshwater ecosystems. Biofilm can therefore, indirectly regulate pelagic primary productivity; however, they can also directly regulate the distribution and primary productivity of submerged aquatic plants (see next section).

\subsubsection{Regulation of Submerged Vegetation}

Biofilm communities have the ability to regulate nutrient dynamics in freshwater ecosystems and prevent detrimental effects associated with excessively high primary productivity. However, epiphyton (i.e., biofilm growing specifically on living plant material) has been suggested to have a detrimental effect on the host plant (Sand-Jensen 1977) and may be a key driver of the abundance and depth distribution of submerged aquatic plants (Phillips et al. 1978; Sand-Jensen \& Borum 1984). The presence of epiphyton can shade photosynthetic tissue of the underlying aquatic plant by reducing light availability by $>85 \%$ (Sand-Jensen $\&$ Søndergaard 1981) and it can also affect the quality of light (Losee \& Wetzel 1983). Biofilm can 
attenuate light between $400-500 \mathrm{~nm}$ and $650-700 \mathrm{~nm}$, and which correspond with the absorption maxima of chl $a$ and the accessory photosynthetic pigments (Raeder et al. 2010). Epiphyton may also act as a physical barrier on aquatic plants and prevent the uptake of inorganic carbon from surrounding water (Sand-Jensen 1977; Jones et al. 2002). Grazing snails have been shown to reduce epiphyton biomass, benefiting aquatic plants to alleviate the burden of attached biofilm (Bronmark et al. 1992). The snail-biofilm-aquatic plant interaction is one of the many potential pathways that facilitate the incorporation of biofilms into other compartments of freshwater food webs (and see next section).

\subsubsection{Energy Input to Food Webs}

Primary productivity associated with phytoplankton is usually assumed to form the photoautotrophic base of freshwater ecosystems, however, biofilm primary production can equal, and sometimes surpass, that of pelagic phytoplankton (Vadeboncoeur \& Steinman 2002). Benthic invertebrates such snails, caddisflies, crayfish, chironomids, and mayflies, are common grazers of biofilm (Mazumder et al. 1989; Bronmark et al. 1992; Blumenshine et al. 1997).

Food web models incorporate common biofilm grazers and have suggested the pervasive and influential role of biofilm in freshwater food webs (Havens et al. 1996). Unlike food web models, stable isotope analysis can reveal the resource pool (i.e., pelagic phytoplankton versus biofilm) and its relative contribution in supporting a consumer (Hecky \& Hesslein 1995; Middelburg 2014). Stable isotope analysis of primary producers uses ratios of ${ }^{13} \mathrm{C}:{ }^{12} \mathrm{C}$ (i.e., $\delta^{13} \mathrm{C}$ calculated relative to the standard Pee Dee Belemnite) to trace carbon through the food webs because of the difference in carbon preference between phytoplankton and biofilms. Phytoplankton discriminately use ${ }^{13} \mathrm{C}$ and thus produce a $\delta^{13} \mathrm{C}$ ratio between -24 and $-38 \%$, whereas biofilm are not as selective and thus have a $\delta^{13} \mathrm{C}$ ratio between -16 and $-30 \%$ (France 1995). Despite its weaknesses (Vadeboncoeur \& Steinman 2002), stable isotopes have demonstrated the importance of biofilm by analyzing $\delta^{13} \mathrm{C}$ in invertebrate grazers (Bunn \& Boon 1993; Hecky \& Hesslein 1995; Bunn et al. 2003; Lau et al. 2009) and top predators like fish (Bunn \& Boon 1993; Kline Jr et al. 1993; Hecky \& Hesslein 1995; Hobson \& Welch 1995; Bootsma et al. 1996; Yoshii 1999; Campbell et al. 2000; James et al. 2000; Vander Zanden \& Vadeboncoeur 2002). The transfer of energy (via organic carbon) between trophic levels represents a central paradigm in food web studies, however, the transfer of certain nutritionally- 
important compounds, such as essential fatty acids, can be more efficient than bulk carbon (Gladyshev et al. 2011) and therefore, can be of key importance to trace in freshwater food webs.

\section{$\underline{1.5}$ Fatty Acids and Freshwater Ecosystems}

In general, fatty acids (FA) are carboxylic acids with an aliphatic (i.e., hydrocarbon) tail; variation between FA species are determined by carbon number and degree and position of saturation (i.e., double bond) in the hydrocarbon chain (CBN 1977). At a cellular level, FA have a wide range of critical functions including energy storage as triacylglycerols (Schönfeld \& Wojtczak 2016), cellular membrane composition as phospholipids (Ibarguren et al. 2014), and cell-to-cell communication (Glatz \& Luiken 2015). Fatty acids can be broadly categorized based on the number of carbon atoms in the hydrocarbon chain. Short-chain fatty acids (SC-FA) typically contain one to seven carbon atoms while long-chain fatty acids (LC-FA) are characterized by $\geq 18$ carbon atoms. The presence or absence of double bonds in the hydrocarbon chain can further categorize FA. Saturated fatty acids (SFA) have only single bonds between all carbons while unsaturated fatty acids (UFA) have single and at least one double bond. The degree of saturation (i.e., number of double bonds) can be used to further categorize UFA. Monounsaturated fatty acids (MUFA) contain only one double bond and polyunsaturated fatty acids (PUFA) contain two or more double bonds. Highly saturated fatty acids (HUFA) are a subset of PUFA that contain $>2$ double bonds in an acyl chain with $\geq 20$ carbon atoms (e.g., Brett \& Müller-Navarra 1997), however, in my review PUFA, the more general term, will be primarily used. Fatty acids with more than two double bonds (i.e., PUFA) can also be classified according to the position of the first double bond from the methyl end such that omega-3 (herein referred to as n-3) and omega-6 (herein referred to as n-6) PUFA have their first double bond at the $3^{\text {rd }}$ and $6^{\text {th }}$ carbon, respectively, from the methyl end.

Fatty acids are commonly referred to in literature using the notion $A: B n-x$; where $A$ represents the total number of carbon atoms, $B$ represents the number of double bonds and $x$ represents carbon position of the first double bond, relative to the terminal methyl group. For example, arachidonic acid (ARA; 20:4n-6) is an omega-6 long-chain polyunsaturated fatty acid (LC-PUFA) with 20 carbons in its acyl chain, four double bonds, with the first double bond appearing on the sixth carbon from the methyl end. Independent of its chemical structure, essential fatty acids (EFA) are classified due to the inability of an organism to biosynthesize an 
adequate quantity of a certain FA to meet physiological needs, and therefore, they must obtain it from their diet. The term can apply to different FA depending on the focus organism (Parrish 2009), however, in aquatic ecosystems LC-PUFA such EPA (eicosapentaenoic acid; 20:5n-3) and DHA (docosahexaenoic acid; 22:6n-3) are the most studied EFA due to their importance in higher trophic levels (discussed later). Fatty acids (e.g., EPA and DHA) are important nutritional components in freshwater ecosystems as they are mainly produced at the base of the food web and transferred and retained by higher trophic levels due to their physiological benefits.

\subsubsection{Fatty Acid Production in Phytoplankton}

Primary productivity supports and influences complex food webs within freshwater ecosystems (Danielsdottir et al. 2007). In eukaryotic algae, de novo synthesis of FA starts with their ability to use solar energy to fix $\mathrm{CO}_{2}$ into carbohydrates, which are further processed into acetyl-CoA (coenzyme A), a critical precursor for FA synthesis in the chloroplast (Guschina \& Harwood 2006). Through a series of two-carbon chain-elongation reactions in the stroma, catalyzed by acetyl-CoA carboxylase (ACCase) and fatty acid synthase (FAS), palmitic (16:0) and stearic acid (18:0) are synthesized (Mishra 2015). These two critical SFA produced in the chloroplast can be subject to further elongation by elongases or to the addition of double bonds by highly-specific desaturases. Palmitic acid is typically converted to hexadecatrienoic acid (16:3n-6), via several soluble desaturases in the stroma, and incorporated into membrane lipids (Mishra 2015). Stearic acid is converted to oleic acid (18:1n-9) - also within the stroma - but is then transported to the endoplasmic reticulum to undergo a series of elongation and desaturation reactions in two possible pathways, an n-3 or $n-6$, to produce a wide range of PUFA (Guschina \& Harwood 2006). The major difference in the mechanism of FA biosynthesis in cyanobacteria, a photosynthetic prokaryotes, is that all reactions occur in the cytoplasm of the cell due to the lack of membrane-bound organelles (Lem \& Stumpf 1984). Through a process similar to eukaryotic algae, cyanobacteria FA synthesis also produces both palmitic and stearic acid (Lem \& Stumpf 1984).

Although there are similarities in the FA synthesis between photosynthetic eukaryotic and prokaryotic organisms, composition/makeup of individual FA molecules (also called the FA profile) can vary dramatically between phytoplankton taxa. This principle underlies the use of FA as indicators of community composition of phytoplankton in both freshwater and marine 
food webs; either by the presence of specific FA (i.e., biomarkers) or their overall FA composition (Lang et al. 2011; Taipale et al. 2013). For example, multivariate analyses show that taxonomic groups explain three to four times more variation in FA profiles between classes of phytoplankton than environmental conditions (Galloway \& Winder 2015). In this context, diatoms generally produce high proportions of PUFA, especially EPA (Kates \& Volcani 1966; Volkman et al. 1989; Ahlgren et al. 1992; Dunstan et al. 1993; Viso \& Marty 1993; Patil et al. 2007; Lang et al. 2011). Cryptophytes can also have high proportions of EPA (Ahlgren et al. 1992; Peltomaa et al. 2017) while dinoflagellates (Harrington et al. 1970; Ahlgren et al. 1992; Mooney et al. 2007; Lang et al. 2011) and chrysophytes (Taipale et al. 2013) can produce high proportions of DHA. On the contrary, chlorophytes and cyanobacteria generally do not produce high quantities of EPA or DHA (Guedes et al. 2011; Strandberg et al. 2015). Cyanobacteria typically contain very low content of FA >C18 (Ahlgren et al. 1992; Iliev et al. 2011; Lang et al. 2011), however, several species have been shown to produce $\mathrm{FA} \leq \mathrm{C} 18$ with up to four double bonds (Parker et al. 1967; Holton et al. 1968; Sallal et al. 1990; Murata et al. 1992; Gugger et al. 2002; Rezanka et al. 2003). Cyanobacteria may not be able to produce PUFA >C18 because they only possess 4 specific types of desaturate enzymes and can be grouped based on the position of double bonds (Kenyon et al. 1972; Murata et al. 1992; Chi et al. 2008; Los \& Mironov 2015), however, certain eukaryotic algae have been shown to possess $>20$ desaturases, all with high levels of specificity (Li-Beisson et al. 2015). It is evident that there is variation in phytoplankton FA composition, especially with respect to EPA and DHA, which are important in aquatic ecosystems considering higher trophic levels depend on their diet to obtain these EFA for optimal growth and reproduction.

\subsubsection{Essential Fatty Acids in Freshwater Pelagic Food Webs}

Pelagic phytoplankton are significant sources of carbon to higher trophic levels (Brett et al. 2009) but their nutritional quality, which can be determined by FA composition, can also play an important role in regulating higher trophic levels (Kainz et al. 2004). In addition, the FA composition of higher trophic levels often mirror the FA composition of their diet (Lee et al. 1971; Brett et al. 2006), suggesting the importance of EFA. Finally, two LC-PUFA (EPA and DHA) are considered essential in aquatic systems as they are required for optimal health in 
higher trophic levels, which has mostly been demonstrated in primary consumers (i.e., zooplankton) but do influence all organisms within, and reliant on, aquatic ecosystems.

The influence of EFA on the health of zooplankton has been extensively studied. Early studies demonstrated certain FA, especially EPA and DHA, were necessary for growth and reproduction of zooplankton (Conklin \& Provasoli 1977; Støttrup \& Jensen 1990; MuellerNavarra 1995; Müller-Navarra et al. 2000). The model organism, Daphnia, was used to show a direct link between EPA and zooplankton health by artificially supplementing their diet with lipid emulsions (Demott \& Müller-Navarra 1997; Weers \& Gulati 1997; Boersma et al. 2001; Park et al. 2003) and liposomes (Ravet et al. 2003, 2012; Denoux et al. 2017). EPA has been identified as the primary limiting PUFA for Daphnia growth (von Elert 2002, 2004; MartinCreuzburg et al. 2008) and reproduction (Becker \& Boersma 2003, 2005; Wacker \& MartinCreuzburg 2007). Higher content of EPA are common among field-captured cladocerans while copepods generally also accumulate DHA, suggesting a higher taxa-specific physiological requirement of this PUFA (Ballantyne et al. 2003; Kainz et al. 2004; Persson \& Vrede 2006; Smyntek et al. 2008; Ravet et al. 2010; Burns et al. 2011; Gladyshev et al. 2015; Hiltunen et al. 2015). Zooplankton are unable to synthesize linoleic acid (LNA) and $\alpha$-linolenic acid (ALA), key PUFA precursors, de novo (Moreno et al. 1979), however, when exposed to stressors (e.g., temperature) they can biosynthesize EPA (Koussoroplis et al. 2014) and DHA (Desvilettes et al. 1997a; Koussoroplis et al. 2014) from ALA. In general, low rates $(<2 \%)$ of de novo FA synthesis (Goulden \& Place 1990, 1993) and low elongation and desaturation products (Bell et al. 2007) suggest that biosynthesized quantities are not sufficient for optimal growth (Weers et al. 1997; von Elert 2002) and, therefore, PUFA demands of zooplankton are suggested to be mostly met by dietary sources.

As with most vertebrates, PUFA are essential dietary components in maintaining fish growth and health, especially EPA and DHA (Bell et al. 1986; Lall \& Lewis-McCrea 2007; Tocher 2010). In fish, FA are used for energy storage (released via $\beta$-oxidation), reproduction, membrane structure and function, and hormone production (Tocher 2010). Laboratory studies have demonstrated that PUFA-enriched zooplankton can increase the growth and survival rates of freshwater fish (Verreth et al. 1994; Abedian Kennari et al. 2007; Gholami 2010; Fereidouni et al. 2013). Fish, like all vertebrate, can biosynthesize de novo both palmitic (16:0) and stearic acid (18:0) in the mitochondria (Sargent et al. 2003) and can also desaturate both to yield 16:1n- 
7 (palmitoleic acid) and 18:1n-9 (oleic acid) in the endoplasmic reticulum (Brenner 1974). Vertebrates, including fish, lack two omega-3 desaturates ( $\Delta 12$ and $\Delta 15)$ that prevent them from converting 18:1n-9 to 18:2n-6 (LNA), and from there, to 18:3n-3 (ALA), a key precursor of EPA and DHA (Tocher 2003). Early studies based on a limited number of species suggested that freshwater fish are able to elongate and desaturate dietary LNA and ALA to produce EPA and DHA, whereas marine fish were not able and therefore, require dietary EPA and DHA (Owen et al. 1975; Watanabe 1982), however, this generalization may not apply to all fish species. Diet has, however, been shown to play a key role in FA synthesis in freshwater fish, as rates of lipogenesis are typically depressed by dietary lipids (Shikata \& Shimeno 1994; Shimeno et al. 1995, 1996; Alvarez et al. 2000), and vice versa (Brauge et al. 1995). The role of PUFA within a pelagic phytoplankton-zooplankton-fish food web has been thoroughly studied, however, the role of biofilm in PUFA production and transfer has been relatively less studied, despite its importance to freshwater food webs.

\subsubsection{Essential Fatty Acid Production and Transfer in Freshwater Biofilm}

In freshwater ecosystems, biofilm microorganisms can belong to the same genus as pelagic microbes but simply occupy a different ecological niche. As such, a strong relationship exists between pelagic phytoplankton and algae in biofilms (Sand-Jensen \& Borum 1991). Community composition of both have shown to be highly correlated (Roeder 1977) as planktonic phytoplankton can settle onto biofilm communities and biofilm microbes can be released into the water column (Tekwani et al. 2013; Jäger \& Borchardt 2018). The close relationship between pelagic phytoplankton taxa and PUFA profiles (as discussed earlier) also exists within biofilm communities (McIntire et al. 1969). Biofilm dominated by diatoms tend to have higher PUFA content, especially EPA (McIntire et al. 1969; Torres-Ruiz et al. 2007; Sushchik et al. 2010; Guo et al. 2015) while cyanobacteria-dominated biofilm communities tend to have low EPA and DHA content but may contain $\leq$ C18 PUFA, and especially 18:2n-6 (Parker \& Leo 1965; Sushchik et al. 2010; Guo et al. 2015). Similarly, aquatic fungi can produce C18 PUFA, specifically linoleic and oleic acid (Libkind et al. 2008), which are observed in high proportions in yeast-dominated biofilm (Timke et al. 2005). Finally, odd-chained FA are an indicator of aquatic bacteria (Desvilettes et al. 1997b), however, bacterial biofilms can also contain high proportions of palmitic and vaccenic acid (Chao et al. 2010). 
The transfer of essential PUFA (i.e., EPA and DHA) from biofilm to invertebrate grazers (Torres-Ruiz et al. 2007; Guo et al. 2016a) has been documented and thus establishing that biofilm are important components of invertebrate diets (Feminella \& Hawkins 1995). PUFA are important for the growth and development of aquatic insects (Stanley-Samuelson et al. 1988; Torres-Ruiz et al. 2010). Insect larvae typically accumulate PUFA in their tissues, especially EPA (Hanson et al. 1985; Bell et al. 1994; Ghioni et al. 1996; Sushchik et al. 2003a; Makhutova et al. 2011; Guo et al. 2016b) suggesting a high physiological requirement to maintain optimal health. Some insect larvae have been shown to synthesize LC-PUFA from C18 PUFA precursors (Hanson et al. 1983; Goedkoop et al. 2007), while other species show a limited ability to biosynthesize PUFA, especially EPA (Torres-Ruiz et al. 2010). Amphibiotic insects are important nutritional vectors across the aquatic-terrestrial boundary as they feed and develop as larvae in aquatic ecosystems but emerge as adult insects into surrounding terrestrial ecosystems. The comparison of FA profiles of both stages (i.e., aquatic larvae and terrestrial adult) may be taxa-specific as some families have been shown to be similar (Sushchik et al. 2013) whereas others seem to differ (Gladyshev et al. 2011; Borisova et al. 2016; Makhutova et al. 2017), especially when considering EPA and DHA contents. Compared to terrestrial insects, which typically have little or no EPA and DHA, emerging insects typically have high EPA and DHA content (Buckner \& Hagen 2003; Wang et al. 2006; Hixson et al. 2015). Aquatic emerging insects also transfer substantial quantities of these LC-PUFA to terrestrial ecosystems (Gladyshev et al. 2009, 2011a; Borisova et al. 2016; Martin-Creuzburg et al. 2017; Popova et al. 2017), which then provide essential PUFA to riparian predators such as birds (Twining et al. 2016) and spiders (Fritz et al. 2017). It is thus reasonable to suggest that EFA in biofilms are directly important to aquatic food webs, and indirectly important to surrounding terrestrial food webs, and therefore, factors that affect biofilm quantity and nutritional quality are important in determining EFA availability for both ecosystems.

\subsection{The Influence of Environmental Factors on Quantity and Nutritional Quality of Freshwater Biofilms}

Biofilms are present in all types of aquatic environments (e.g., freshwater and marine systems, wetlands, and man-made systems), each with distinct combinations of environmental factors. Physical (flow rate, light intensity and quality, temperature, oxygen, salinity, etc.), 
chemical ( $\mathrm{pH}$, nutrient availability, organic matter composition), and biological (grazing) factors can influence biofilm development. Light, nutrients, and temperature are critical environmental factors that can determine the structure, chemical composition, and function of aquatic biofilms (Mora-Gómez et al. 2016). The following section will discuss important abiotic factors (i.e., light, nutrients, and temperature) that affect both the quantity and nutritional quality of freshwater biofilms, with a focus on their impact on LC-PUFA (e.g., EPA and DHA).

\subsubsection{Light}

Light is a fundamental abiotic factor for all photosynthetic organisms including those in aquatic ecosystems (Kirk 1994) and the main predictor for the presence of photoautotrophs in aquatic biofilms (Park et al. 2008). Photosynthetically active radiation (PAR), the electromagnetic radiation between 400 and $700 \mathrm{~nm}$, is an important source of energy for photosynthesis as photosynthetic pigments (i.e., chlorophylls and accessory pigments) have peak energy absorption in this range (McCree 1981; Mõttus et al. 2011). Pelagic phytoplankton can differ in their pigment composition and therefore, their response to underwater irradiance can be taxa-specific (Richardson et al. 1983; Langdon 1988). In general, diatoms require relatively low levels of light while chlorophytes typically require higher levels to dominate phytoplankton communities (Sheath et al. 1986; Steinman \& McIntire 1987; Langdon 1988). Light generally increases growth rates of photosynthetic organisms (Dauta et al. 1990) to a certain point, after which photoinhibition and photooxidation cause growth inhibition and even cell death, at very high-light intensities (Powles 1984). Light penetration within a biofilm depends heavily on its structure (e.g., thickness) but generally light irradiance and PAR decreases closer to the substrate and away from the light source (Meulemans 1987; Dodds 1992; van Dijk 1993; Kühl \& Jorgensen 1994; Dodds et al. 1999). The light intensity experienced by the photosyntheticallyactive portion of biofilm proportionally influences the depth at which they grow, such that, the higher the intensity the deeper photosynthesis can occur (Schnurr \& Allen 2015). Although some algal species can migrate in response to light (Fauré-Fremiet 1951; Round \& Eaton 1966), the effect of light on freshwater biofilm also depends on natural variation in light quantity and quality.

The underwater light spectra (i.e., light quantity and quality) that aquatic biofilms are exposed to in freshwater ecosystems can depend on many factors. Terrestrial vegetation (i.e., leaf 
canopy of overhanging trees) can shade and effectively block a significant portion of incoming irradiation, especially in small ponds and streams (Hill et al. 1995). The characteristics of surrounding terrestrial vegetation is important in determining light availability for streams (Davies-Colley \& Quinn 1998; Melbourne \& Daniel 2003); for example, complex old-growth forests typically have frequent canopy gaps that increase light availability for stream biofilm communities (Warren et al. 2013). Logging of adjacent vegetation along a stream can increase light levels, water temperature, and grazer abundance (Kiffney et al. 2003), however, light was isolated as the main regulator of biofilm biomass in these types of manipulation studies (Kiffney et al. 2004). Light is also attenuated and modified through the water column as light is scattered/absorbed by water, dissolved organic matter, suspended inorganic particles, and pelagic phytoplankton (Gallegos \& Moore 2000). The rate at which light availability decreases with depth, quantified by light attenuation coefficients (i.e., $\mathrm{k}_{\mathrm{d}}$ ), varies between systems but typically increases with pelagic nutrient concentrations (Seekell et al. 2015), turbidity (Davies-Colley \& Nagels 2008), and pelagic primary productivity (Schanz 1985).

The photosynthetic rate of biofilm follows a non-linear relationship with light; a linear increase at low levels of irradiance, a plateau at mid-levels as light becomes saturated and photosynthesis begins to peak followed by an asymptotic maximum or a decrease at high levels due to photoinhibition (Hill 1996). In biofilm, photosynthetic saturation typically occurs when irradiance is between 100 and $400 \mu \mathrm{mol} \cdot \mathrm{m}^{-1} \cdot \mathrm{s}^{-1}$, whereas photoinhibition typically occurs when irradiance is above $600 \mu \mathrm{mol} \cdot \mathrm{m}^{-1} \cdot \mathrm{s}^{-1}$ (Hill 1996). The irradiance value at which both photosynthetic saturation and photoinhibition occur depend on the light environment of the biofilm (Boston \& Hill 1991; Hill \& Boston 1991; Dodds et al. 1999), however, communities may also be able to adapt their photosynthetic rates to shading (McIntire \& Phinney 1965). In well-developed biofilm (i.e., many layers of cells), only algae cells near the biofilm-water interface may experience photoinhibition, while cells in the layers below remain protected (Sand-Jensen \& Revsbech 1987; Hill \& Boston 1991). Biofilm in freshwater ecosystems may regularly experience irradiance values that may cause photoinhibition due to daily (DeNicola et al. 1992) and seasonal (Hill \& Dimick 2002) variation, however, natural biofilms can produce protective accessory pigments, like carotenoids, that facilitate their adaptation to changing light environments (Laviale et al. 2009; Sánchez et al. 2013) and, therefore, the relationship between light and biofilm biomass is typically positive. 
In streams, adjacent terrestrial vegetation plays a key role in determining light availability to freshwater biofilm and therefore influence biomass, usually measured by ash-free dry mass (AFDW) or chl $a$ content. Several studies have demonstrated substantially higher biofilm biomass in open sites compared to shaded sites (Lowe et al. 1986; Hill \& Knight 1988; Feminella et al. 1989; Hill \& Harvey 1990; DeNicola et al. 1992; Steinman 1992; Bourassa \& Cattaneo 2000; Larned \& Santos 2000; Mosisch et al. 2001; Roberts et al. 2004; Proia et al. 2012). In addition, artificially illuminating stream biofilm leads to higher biomass than open canopy, closed canopy, and artificially-shaded treatments (Wellnitz et al. 1996). Leaves on overhanging trees not only block irradiance, they also absorb blue (450 - $495 \mathrm{~nm})$ and red (620 $750 \mathrm{~nm}$ ) wavelengths (Federer \& Tanner 1966), resulting in reduced quantities of high-quality PAR available for aquatic biofilms (Hill \& Dimick 2002). Turbidity, due to small particles, in streams also reduces light penetration and reduces biofilm biomass (Davies-Colley et al. 1992). In lakes, adjacent trees affect the light conditions of a relatively small area around the perimeter; instead, the water column depth and dissolved organic content (humic acids) are the main factors that influences underwater light spectra available for biofilm. An inverse relationship between the biomass of pelagic phytoplankton and biofilm in lakes (Hansson 1988, 1992; Sand-Jensen \& Borum 1991; Havens et al. 2001a; Vadeboncoeur et al. 2001) suggests the role of pelagic phytoplankton in reducing PAR for underlying biofilm communities. In marine habitats, light intensity may be more important than light quality (Dring 1981; Ploug et al. 1993). Light manipulation of entire lakes is logistically difficult, however, the positive effect of light on biofilm biomass has been shown in large experimental channels clear of canopy cover at the outlet of a lake (Bourassa \& Cattaneo 2000).

Biofilm may also experience high levels of ultraviolet (UV) radiation, especially UV-A (315 - $400 \mathrm{~nm})$ and UV-B (280 - $315 \mathrm{~nm})$, both which can reach several meters below the surface, especially in clear waters (Karentz \& Lutze 1990; Vincent \& Roy 1993). Ultraviolet radiation can inhibit photosynthesis and damage DNA and can reduce biofilm biomass (Bothwell et al. 1993, 1994; Vinebrooke \& Leavitt 1996; Rader \& Belish 1997). Biofilm collected from higher altitudes have lower AFDW, chl $a$ content, and bacterial biodiversity (Bartrons et al. 2012; Wilhelm et al. 2013, 2015), however, these communities typically produce higher levels of protective accessory pigments (e.g., carotenoids) to mediate damages associated with higher levels of UV radiation (Vinebrooke \& Leavitt 1999). The migratory ability of some epipelic 
microorganisms in response to light (Happey-Wood 1988; Vincent et al. 1993; Serôdio et al. 1997) has been proposed as a mechanism to explain the increase in epipelic biomass due to UVA radiation (Vinebrooke \& Leavitt 1999). The clear positive relationship between light and biofilm biomass has been examined thoroughly, however, the effect of light on the nutritional quality of biofilm has only recently begun to be studied.

Light can regulate the presence of algae in biofilm and the overall biomass, however, it also plays a major role in determining nutritional quality. Light has been shown to have varying effects on stoichiometry (C, N or P content) of biofilm, ranging from a positive (Qin et al. 2007; Sanches et al. 2011; Kohler et al. 2012) to a negative effect (Hillebrand et al. 2004), to no effect at all (Frost \& Elser 2002; Hill et al. 2009; Liess et al. 2009). Recently, EFA have been used an indicator of nutritional quality of biofilm, due to the importance of EPA and DHA for higher trophic levels. Light plays a role in FA production and has recently been shown to affect the FA composition of biofilms, including EFA. For example, shaded biofilm exhibited higher proportions of PUFA compared to biofilm from open sites (Guo et al. 2015, 2016a). High light levels also tend to increase the overall FA content in biofilm (Cashman et al. 2013). Elevated light increases the proportion of SFA and MUFA and decrease proportions of PUFA (Hill et al. 2011). In-situ canopy manipulation experiments demonstrate that high light increases MUFA and $<20 \mathrm{C}$ PUFA but reduce SFA and, more importantly, reduces the proportion of $\geq 20 \mathrm{C}$ PUFA such as EPA and DHA (Cashman et al. 2013). Pelagic phytoplankton exhibit a similar reduction in PUFA when exposed to high light levels in marine (Thompson et al. 1990, 1993; Mock \& Kroon 2002; Leu et al. 2010) and freshwater (Wacker et al. 2016) systems, likely as a mechanism of photo-acclimatization. In general, low light levels typically increases the proportion of PUFA in pelagic phytoplankton while high light levels stimulates the synthesis of SFA (Guschina \& Harwood 2006). Light (i.e., open versus shaded canopy) has also shown to have minimal effects on all measured FA in biofilm (Guo et al. 2016c). However, nutrients may have played a role in regulating biofilm response to light treatments (briefly discussed in the next section) due to their role on biofilm growth.

\subsubsection{Nutrients}

The importance of elemental nutrients, especially phosphorus and nitrogen, for freshwater pelagic phytoplankton has been thoroughly studied (Hecky \& Kilham 1988; Elser et 
al. 1990, 2007). Phosphorus and nitrogen are both essential nutrients as they are constituents in all cells and are necessary for cell development and growth. Phosphorus plays a key role in several cellular functions including: storing and transcribing genetics information (i.e., DNA and RNA), structural membranes (i.e., phospholipids), production of energy (i.e., adenosine triphosphate (ATP) and nicotinamide adenine dinucleotide phosphate (NADPH)), and regulation of proteins (i.e., phosphorylation; Lin et al. 2016). Nitrogen is required in the production of amino acids, the building blocks for all proteins that are vital for cellular function and growth (Antia et al. 1991). In aquatic ecosystems, the most bioavailable forms of phosphorus are in orthophosphate ions $\left(\mathrm{PO}_{4}{ }^{3-}\right)$ in solution and are usually quantified as molybdate-reactive (MRP) or soluble-reactive phosphorus (SRP; Reynolds \& Davies 2001). Nitrogen can be found as ammonium $\left(\mathrm{NH}_{4}{ }^{+}\right)$, nitrite $\left(\mathrm{NO}_{2}^{-}\right)$and nitrate $\left(\mathrm{NO}_{3}{ }^{-}\right)$, all of which are soluble, inorganic, and bioavailable (Bowden 1987). Typically, phosphorus has been suggested as the limiting nutrient for primary productivity in freshwater systems (Hecky \& Kilham 1988). However, a metaanalysis revealed that lake phytoplankton and stream biofilm are equally limited (co-limited) by phosphorus and nitrogen while lake biofilm are more limited by phosphorus (Elser et al. 2007).

Phosphorus and nitrogen are commonly cited as the two most essential nutrients in freshwater ecosystems, however, other nutrients have been suggested as regulators of biofilm growth, but to a lesser extent. These include silica (specifically for diatoms-dominated biofilms; Fields et al. 2014), carbon (Fairchild et al. 1989; Fairchild \& Sherman 1993), trace metals (Wuhrmann \& Eichenberger 1975; Winterbourn 1990), magnesium (Song \& Leff 2006), and vitamin $B_{12}$ (Hoflmann 1990). Determining the direct effect of nutrients on biofilm in natural systems is difficult due to the influence of a wide range of other mitigating uncontrolled variables such as light and temperature (Welch et al. 1988; Biggs \& Close 1989; Duncan \& Blinn 1989; Hansson 1992; Fairchild \& Sherman 1993; Rosemond 1994). However, supplementation studies have been an essential tool to directly measure the influence of phosphorus and nitrogen on biofilm (discussed below).

The importance of elemental nutrients on biofilm growth may have first been examined when three 125-pound bags of commercial fertilizer (with a N:P:K ratio of 4:12:6) were placed into a river and thick filamentous algae biofilm was observed downstream one year later, while upstream substrates were barren (Huntsman 1948). In streams, phosphorus increases biofilm biomass, as indicated by increases in chl $a$ content (Stockner \& Shortreed 1978; Horner \& Welch 
1981; Horner et al. 1983; Peterson et al. 1985, 1993; Stanley et al. 1990; Winterbourn 1990), algal cell biomass (Pringle \& Bowers 1984; Bothwell 1989; Horner et al. 1990), AFDW (Elwood et al. 1981), and growth rates (Bothwell 1985). Nitrogen has also been shown, to a lesser extent, to increase biofilm biomass in streams (Grimm \& Fisher 1986; Hill \& Knight 1988; Winterbourn 1990; Lohman et al. 1991; Peterson \& Grimm 1992). Similarly, both nutrients can dictate biofilm biomass in lakes (Fairchild et al. 1985; Marks \& Lowe 1989, 1993). Few studies have pinpointed the growth-limiting concentration of phosphorus (Bothwell 1988; Horner et al. 1990) or nitrogen (Grimm \& Fisher 1986; Lohman et al. 1991), however, ambient concentrations tend to be low suggesting naturally-occurring biofilm communities in freshwater systems are nutrientdeficient and any enrichment may induce increase in growth. Although not the focus of enrichment studies in biofilms, phosphorus enrichment may also lead to dominance of cyanobacteria (Elwood et al. 1981; Fairchild et al. 1985), however, other studies have reported no change algal composition (Stockner \& Shortreed 1978). Interestingly, the interactive effect of both nutrients can have a synergistic effect on the algal biomass of biofilm (Elser et al. 1990, 2007), especially in high light conditions (discussed below).

In several supplementation studies, the combined effect of phosphorus and nitrogen was more substantial than the individual effects (Stockner \& Shortreed 1978; Pringle \& Bowers 1984; Pringle 1987; Stanley et al. 1990; Winterbourn 1990; Burton et al. 1991; Lohman et al. 1991). Despite the importance of both, nutrient enrichment without sufficient light may limit the manifestation of an effect on overall biofilm growth (Hill \& Knight 1988; Winterbourn 1990). The increase in autotrophic biomass with the addition of both nutrients may be further increased in high light conditions (e.g., Rosemond 1993; Guariento et al. 2011), suggesting that both light and nutrients may co-limit algal biomass (Taulbee et al. 2005; Hill \& Fanta 2008). Interestingly, the addition of nutrient and light had a synergistic negative effect on non-autotrophs (Guariento et al. 2011), which has also been observed with the addition of phosphorus (Scott \& Doyle 2006) and nitrogen (Cook et al. 2007), individually. It is suggested that the nutrient addition in highlight conditions may alleviate nutrient and light limitation of algal cells leading to a decoupling of autotrophic/heterotrophic interactions, ultimately leading to nutrient limitation in bacterial cells (Guariento et al. 2011). The interactive effect of nutrients and light has led to the light:nutrient hypothesis ( $\mathrm{LNH}$ ), which attempts to determine the balance between light and nutrients required for phytoplankton (Sterner et al. 1997) and biofilm growth (Fanta et al. 2010). 
Nutrients can evidently increase the overall and algal component of freshwater biofilm, which can also greatly affect the nutritional quality of biofilm (discussed below).

Biofilm found in nutrient-rich waters typically have lower carbon:phosphorus (C:P) and carbon:nitrogen $(\mathrm{C}: \mathrm{N})$ ratios (Kohler et al. 2012) than biofilm from nutrient-poor waters.

Nutrient enrichment experiments typically demonstrate a reduction of both ratios (Peterson et al. 1993; Stelzer \& Lamberti 2001; Hillebrand et al. 2004), suggesting the high incorporation of both nutrients into biofilms. Nutrients also play a key role in the FA composition of algae, and therefore, can influence the nutritional quality of algal biofilm. In pelagic algae, high nutrient levels cause an increase in the content of PUFA-rich galactolipids, while nutrient limitation results in SFA-rich triacylglycerols (Guschina \& Harwood 2006). Field observations on water nutrient concentration and biofilm FA demonstrate a negative relationship between nitrogen and SFA and positive relationships with most PUFA (Guo et al. 2015). Laboratory experiments show phosphorus supplementation decreases SFA and MUFA and increases PUFA proportions (Hill et al. 2011). Results from an in-situ manipulation experiment were contradictory as added nutrients had no effect on most FA, except a significant decline in DHA and increase in DHA precursors (Cashman et al. 2013). Other laboratory studies demonstrated an increase in DHA and other PUFA in response to nutrient enrichment (i.e., fertilizer; Guo et al. 2016b). There is also some evidence of an interactive effect of light and nutrients on FA content, especially with essential PUFA such as EPA and DHA (Guo et al. 2015, 2016a; c). Nutrient supplementation and shaded conditions (i.e., low light) also increase PUFA contents (Guo et al. 2016a; c). Studying the effect of nutrients and light, both individually and combined, is essential to understanding the role of abiotic factors on biofilm quantity and quality in natural systems.

\subsubsection{Temperature}

Temperature is the final abiotic factor examined in this review and is a critical driver of metabolism (Clarke \& Johnston 1999; Toseland et al. 2013) and growth (Butterwick et al. 2005; O’Gorman et al. 2016), as well as large-scale nutrient cycling (Friberg et al. 2013; Martínez et al. 2014). The effect of temperature on pelagic phytoplankton has been extensively studied (Eppley 1972; Canale \& Vogel 1974; Li 1980; Grimaud et al. 2017), especially in the context of global warming and understanding and predicting algal blooms (Robarts \& Zohary 1987; Joehnk et al. 2008; Paul 2008; Paerl \& Huisman 2009; Elliott 2012). Optimal temperature may differ between 
species within each phytoplankton class (Hustedt 1956; Admiraal 1976; Coles \& Jones 2000) and even between strains of the same species (Souffreau et al. 2013). At a cellular level, higher temperature increases the kinetic energy of reactions involved in photosynthesis (Nielsen \& Jørgensen 1968; Davison 1991). Algal cells tend to alter their carbon-fixing enzyme and lightharvesting chlorophyll content to compensate for temperature-related changes to enzymatic activity (Yoder 1979; Rhee \& Gotham 1981; Schlesinger \& Shuter 1981; Mortain-Bertrand et al. 1988; Thompson et al. 1992). Higher temperatures also typically increase growth rates (i.e., by reducing doubling times) of bacteria and algae within a biofilm (Bott 1975; Aizaki 1979) and the entire biofilm communities (Aizaki 1979), which directly determines biofilm quantity.

Primary productivity is more closely related to temperature in stream biofilm than in lake or ocean pelagic phytoplankton (Morin et al. 1999). In general, it has been suggested that aquatic biofilms have an optimal growth temperature between 10 and $30^{\circ} \mathrm{C}$ (DeNicola 1996), however, freshwater biofilm have been observed to grow in temperatures ranging from 0 to $75^{\circ} \mathrm{C}$ (Brock 1967; Fogg 1969; Vincent \& Howard-Williams 1986). In freshwater streams, maximum biofilm biomass occurs in spring, fluctuates in summer and fall, with minimal biofilm biomass in winter (Marker 1976; Moore 1977a; b; Sumner \& Fisher 1979; Cox 1990). In freshwater lakes, maximum biofilm biomass is usually observed in spring and late summer/early fall (Castenholz 1960; Round 1961; Klarer \& Hickman 1975; Hickman 1978a; Eloranta 1982), driven by the optimal combination of temperature, nutrients, and light. Higher water temperatures generally increase biofilm biomass in both streams (Patrick 1971; Squires et al. 1979; Descy \& Mouvet 1984) and lakes (Hickman 1974; Eloranta 1982; Patrick et al. 2012; Tarkowska-Kukuryk \& Mieczan 2012; Cao et al. 2014; Mahdy et al. 2015) due to an exponential increase in photosynthetic rates as temperature increases (Stanley \& Daley 1976; DeNicola 1996). The increase is observed with temperatures up to the upper thermal range of aquatic microorganisms (i.e., $\sim 30^{\circ} \mathrm{C}$ ), after which biofilm biomass decreases (Patrick 1971). In studies where temperature had a negative or no effect on biomass (e.g., Shurin et al. 2012), increased grazing pressure due to increased temperature (Hillebrand 2009) may mask the direct impact of temperature on biofilm biomass (Cao et al. 2014). Higher water temperatures have also been shown to increase community metabolism, measured by respiration rates (Kevern \& Ball 1965; Phinney \& McIntire 1965; McIntire 1966; Acuña et al. 2008; Rosa et al. 2013). The increase in community metabolism may explain the positive influence of nutrient supplementation in temperature 
manipulation studies (e.g., Bothwell 1988; Cao et al. 2017). Higher temperatures tend to increase growth of microorganisms within biofilms, up to a certain extent, where a change in phytoplankton species composition can often occur in response to temperature changes in freshwater systems and biofilms.

In pelagic systems, temperature can influence the seasonal succession of pelagic phytoplankton (Richardson et al. 2000; Fietz et al. 2005; Schabhüttl et al. 2013). In general, diatoms have lower optimal growth temperatures than other eukaryotic algae and cyanobacteria (Butterwick et al. 2005; Lürling et al. 2013), partially explaining the dominance of diatoms in spring and potentially fall and by cyanobacteria in the summer. Seasonal patterns of algae in biofilms have also been linked with temperature (Castenholz 1960; Gruendling 1971; Hickman 1978b; Moore 1978; Wehr 1981; Seaburg \& Parker 1983; Admiraal et al. 1984; Cox 1993; Griffith et al. 2002), however other interacting abiotic factors (i.e., light and nutrients) confound clear conclusions about the effect of temperature. Naturally- or experimentally-altered experiments have shown that higher temperatures $\left(>30^{\circ} \mathrm{C}\right)$ tend to cause a microbial community shift of biofilms towards a cyanobacteria-dominated community (Hickman 1974; Klarer \& Hickman 1975; Wilde \& Tilly 1981; Wilde 1982; Lamberti \& Resh 1985; Watermann et al. 1999; van der Grinten et al. 2005; Paerl \& Huisman 2008; Larras et al. 2013) while diatoms dominate at lower temperatures $\left(5-20^{\circ} \mathrm{C}\right.$; Patrick 1971; Klarer \& Hickman 1975; Squires et al. 1979; Wilde \& Tilly 1981; Lamberti \& Resh 1985; Vinson \& Rushforth 1989; Watermann et al. 1999; van der Grinten et al. 2005; Larras et al. 2013). Absolute values discussed above can change between studies (e.g., Patrick et al. 1969) but, generally, these patterns typically hold in freshwater systems. Warmer temperatures, associated with climate change, may increase biofilm biomass, however, the shift in species composition and changes in membrane FA proportions (discussed below) may negatively affect nutritional quality, especially with respect to PUFA.

As discussed, high temperatures can increase growth rates of biofilms, but temperature can also influence the nutritional quality of the microorganisms and therefore, the biofilm as a whole. Biofilm collected along a thermal gradient demonstrated that temperature was negatively correlated with biofilm carbon, nitrogen, and phosphorus content (Moody et al. 2017). Temperature also plays an important role in the FA composition, a key measure of nutritional quality, of microorganisms as higher temperature lead to lower proportions of PUFA like EPA and DHA. Cells respond to changes in temperatures through homeoviscous adaptation; a 
biochemical process which maintains proper membrane fluidity by modifying the proportion of SFA and PUFA in cellular membranes (Sinensky 1974; Hazel \& Williams 1990; Hazel 1995; Ernst et al. 2016; Fig. 1.2). An increase in temperature fluidizes the membrane and a greater proportion of SFA are added in an attempt to increase rigidity, resulting in a reduction of PUFA (Fig. 1.2). Higher temperatures have been observed to reduce PUFA content in phytoplankton (Teoh et al. 2004; Fuschino et al. 2011; Pasquet et al. 2014) suggesting the negative impact of temperature on the nutritional quality of biofilm microorganisms. Seasonal variation in temperature and biofilm PUFA content exist (Sushchik et al. 2010; Honeyfield \& Maloney 2015; Richoux et al. 2018), but the direct effect of temperature is difficult to tease apart from other abiotic factors. Temperature has been suggested as a potential explanation to differences in the proportion of SFA and PUFA in biofilm (e.g., Hill et al. 2011; Cashman et al. 2013; Guo et al. 2015), however, the direct effect of temperature on biofilm nutritional quality (i.e., PUFA) has not been examined thoroughly.
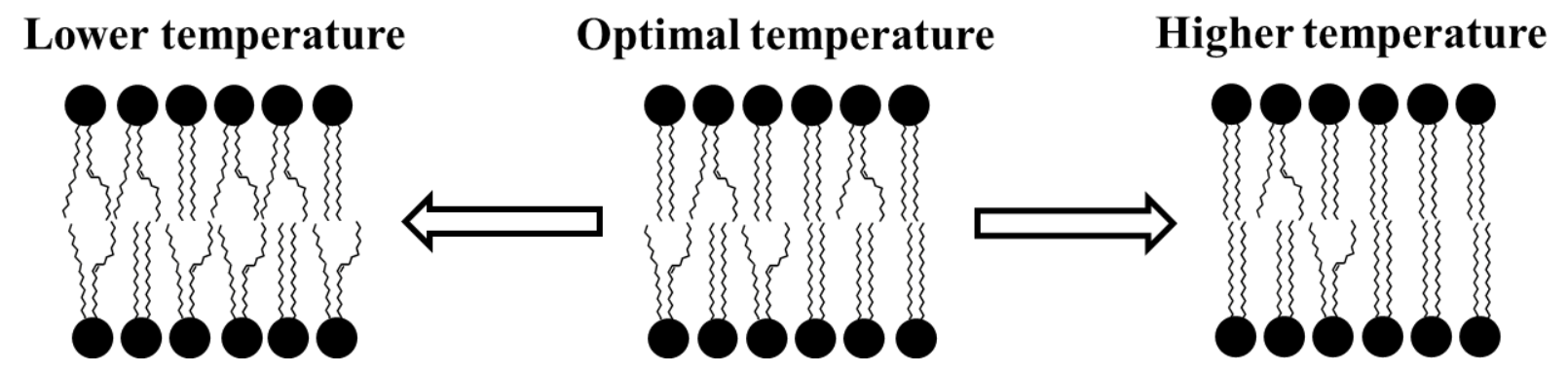

Figure 1.2: Schematic representation of homeoviscous adaptation to different temperatures. Final proportion of saturated (two straight fatty acids) and unsaturated (one straight and one kinked fatty acid) represent the changes in fatty acid proportions due to higher and lower temperatures. Unsaturated fatty acids are substituted in at low temperatures to prevent rigidification while saturated fatty acids at higher temperatures prevent fluidization of membranes.

Algae are key microbes in freshwater biofilms that contribute to their nutritional quality, however, their response to warming temperatures is not fully understood. Warming temperatures may reduce the overall PUFA proportion as microorganisms adapt to changes in membrane fluidity, however, the species composition shift towards a cyanobacteria-dominated community will also reduce PUFA content of biofilm as cyanobacteria typically have little LC-PUFA like EPA and DHA (e.g., Guedes et al. 2011; Strandberg et al. 2015). Therefore, the nutritional quality of freshwater biofilm may be significantly reduced with warmer temperatures. The effect 
of elevated temperature on FA composition in biofilm is not well-understood and merits further study in the context of climate change.

\section{$\underline{1.7 \text { Climate Change }}$}

Climate change can refer to a multitude of long-term (i.e., decades) patterns in the state of Earth's climate, including the frequencies of extreme weather events, frequency and intensity of droughts, changing precipitation patterns, and global warming (Hartmann et al. 2013). Scientists have known about the ability of certain atmospheric molecules to absorb radiation reflected from the surface and re-emit the radiation downwards, warming the lower atmosphere and the surface since the 1800s (Tyndall 1861; Arrhenius 1896). Recently, during the last two decades, scientists have accepted the reality and the anthropogenic causes of global warming (Cook et al. 2013, 2016). An overall increase of $0.89^{\circ} \mathrm{C}$ in global mean surface temperature (GMST) has been observed between 1880 (i.e., immediately after the Industrial Revolution) and 2012, however, a $0.72^{\circ} \mathrm{C}$ increase between 1951 and 2012 suggests that warming has accelerated recently (Hartmann et al. 2013).

The rise in global temperatures is very likely due to the anthropogenic increases of greenhouse gas emissions as atmospheric concentrations of carbon dioxide $\left(\mathrm{CO}_{2}\right)$, methane $\left(\mathrm{CH}_{4}\right)$, and nitrous oxide $\left(\mathrm{N}_{2} \mathrm{O}\right)$ in 2011 were 40\%, 150\%, and 20\% greater, respectively, than in 1750 (Hartmann et al. 2013). This increase in atmospheric greenhouse gases is primarily due to fossil fuel emissions after the Industrial Revolution as current greenhouse gas levels are unmatched for the last 800,000 years (Ciais et al. 2014). Models predicting future global temperatures have become more complex (see Zong-Ci et al. 2013), however, most models vary in their prediction based on future anthropogenic emission rates. The GMST for 2081 - 2100 , relative to 1986 - 2005 , is expected to be $0.3-4.8^{\circ} \mathrm{C}$ warmer, depending on the rate of anthropogenic emissions (Collins et al. 2013). Warming temperatures will affect all ecosystems (Parmesan \& Yohe 2003), however, freshwater ecosystems are especially vulnerable because they are typically small and shallow, relatively isolated, physically fragmented, usually within terrestrial landscapes, and heavily exploited by humans (Downing et al. 2006; Woodward 2009). Freshwater ecosystems are also important biodiversity hotspots; although they only cover $0.8 \%$ of the Earth's surface, they support an estimated 6\% of all described species (Dudgeon et al. 2006)! 


\subsubsection{Freshwater Ecosystems and Climate Change}

The predicted increase in GMST will also increase water temperatures in aquatic ecosystems worldwide as surface water temperature typically correlates strongly with air temperature (McCombie 1959; Morrill et al. 2005). Aquatic ecosystems are expected to have three main ecological responses to warming temperatures: a) shift of species ranges toward higher latitudes (Hickling et al. 2006), b) seasonal shifts in life cycle events and trophic decoupling (Winder \& Schindler 2004), and c) reduced body size (Daufresne et al. 2009). Warming temperatures are directly expected to largely reduce the production of EFA in phytoplankton globally through cellular adaptation of membranes (Hixson \& Arts 2016), which can be considered as another ecological response. Phytoplankton are the main producers of omega-3 LC-PUFA, like EPA and DHA, (Hixson et al. 2015) that are essential for both freshwater and terrestrial food webs, including humans (Zárate et al. 2017). Both homeoviscous adaption and phytoplankton community shifts are predicted to reduce quantities of essential PUFA at the base of freshwater food webs, the ecosystem-wide ramifications of which have not fully been studied.

Controlled and replicated laboratory experiments as well as mathematical models (Woodward et al. 2010) have shown the potential negative effect of higher temperatures on PUFA production in phytoplankton, however, additional field and natural experiments are required to understand the more realistic food web consequences. Analysis of field-collected samples suggest phytoplankton from cold, high-latitude regions produce a disproportionally large portion of the global PUFA stocks (Colombo et al. 2016). Surface waters of shallow ponds have already experienced water temperatures as high as $32.6^{\circ} \mathrm{C}$ (King City, ON; July 2015; see Ellen 2017). Furthermore, model predictions indicate a potential increase in the number of extreme heat days (i.e., number of days $>30^{\circ} \mathrm{C}$; Fischer \& Knutti 2015), which can increase proportions of phytoplankton SFA and MUFA at the expense of PUFA (Maazouzi et al. 2008). The temperature-dependent decrease in the proportion of PUFA has been shown in zooplankton in both laboratory (Masclaux et al. 2012) and field-collection studies (Gladyshev et al. 2011). Some studies have even used natural thermal gradients along rivers to remove confounding variables and have observed similar trends in invertebrates (Gladyshev et al. 2016). Despite the recent studies on the potential effects of climate change on PUFA production in phytoplankton 
and transfer to zooplankton, little effort has been made to confirm laboratory results in natural waterbodies.

\subsection{Focus of Thesis}

My thesis will address two major objectives. The first objective was to measure the potential impact of climate change and PUFA in freshwater food webs, while the second objective was to determine the effect of season and water depth on biofilm quantity and nutritional quality.

The first objective was to provide the first in-situ evaluation of the effects of increasing water temperature on the, a) production, b) transfer, and, c) re-distribution of essential fatty acids (e.g., EPA and DHA) in freshwater ecosystems. Climate change studies predict the potential effects of warming temperatures on freshwater population responses using mathematical models and laboratory experiments while using field experiments to study community and ecosystem responses (Woodward et al. 2010). Laboratory results have shown the reduction of PUFA proportions at warmer temperatures in phytoplankton (e.g., Fuschino et al. 2011; Pasquet et al. 2014) and recent empirical models suggest a $2.5^{\circ} \mathrm{C}$ increase would cause a worldwide reduction of EPA and DHA production by $8.2 \%$ and $27.8 \%$, respectively (Hixson \& Arts 2016). A largescale in-situ temperature manipulation, however, would provide a new conceptualization of the effects of climate change on aquatic ecosystems and critical resources that are required to maintain the health of aquatic and terrestrial organisms.

In order to determine the effect of temperature on PUFA in natural freshwater systems, I attempted to warm one half of a shallow pond $4{ }^{\circ} \mathrm{C}$ above ambient water temperature during the spring-summer months (Chapter 2). Sample collection would assess the effect of warming on a) PUFA production in primary producers (phytoplankton and biofilm), b) PUFA transfer through trophic levels to secondary consumers (zooplankton and insect larvae), and c) PUFA redistribution to adjacent terrestrial systems via emerging insects. Based on previous laboratory experiments, I hypothesized that primary producers (i.e., phytoplankton and biofilm) would produce lower proportions of PUFA, especially EPA and DHA, in the heated treatment. Lowered production will result in lower PUFA proportions in zooplankton and insect larvae and thus, emerging insects in the heated treatment would also have had lower PUFA production. The setup of the in-situ temperature manipulation experiment, subsequent pitfalls, and future considerations 
are discussed in Chapter 2. In summary, the in-situ temperature manipulation experimental setup did not achieve the intended warming effect.

The second objective of my thesis was to examine the effects of season and water depth on biofilm quantity and nutritional quality. Abiotic factors (i.e., light, nutrients, and water temperature) have been shown to affect biofilm quantity and quality, both separately and interactively. Depth can have varying effects on biofilm biomass, depending on the growth substrate, however, artificial substrates have been used to show increased biofilm biomass at shallower depths (Liboriussen \& Jeppesen 2006, 2009; Yang \& Flower 2012; Sánchez et al. 2013; Schroeder et al. 2013; Kreuzinger-Janik et al. 2015). The effect of grazers and nutritional quality of biofilm has also been studied in relation to depth. Specifically, abundance of grazers (e.g., snails, nematodes, rotifers, insect larvae) and the effect of grazing on biofilm can strongly vary along a depth gradient (Schroeder et al. 2013). Nutritional quality of biofilm, measured by stoichiometry ratio of carbon:nitrogen:phosphorus (C:N:P) ratios, has also been shown to increase with increasing water depth (Kahlert et al. 2002). The effect of depth on biomass and stoichiometry ratios have been examined, however, the nutritional quality (i.e., PUFA content) of biofilm grown at different depths have not been studied.

In order to determine the effect of depth on the PUFA content and lipid profile of biofilm, I deployed custom-built biofilm incubators with unglazed ceramic tiles at 6 different depths (25, $50,75,100,125$, and $150 \mathrm{~cm}$ ) below the water surface in a small pond. Biofilm was incubated at the varying depths for 67 days in the summer months and harvested. Tiles were, then, powerwashed and placed at the same depths for another 67 days and then harvested to capture changes in the fall months. I assessed biofilm quantity via confocal laser scanning microscope (CLSM), dry weight (DW), and ash-free dry weight (AFDW). Nutritional quality (PUFA) of biofilm was assessed using gas chromatography (GC). I hypothesized, based on the literature, that biofilm quantity and quality would be lower in summer months and as water depth increases. Results from this experiment may be able to determine the quality as well as the nutritional value of biofilm at different depths.

The content of this thesis can contribute to future of climate change and provide depthrelated baseline nutritional values for further freshwater biofilm research. The climate change work can provide recommendations for future field experiments trying to manipulate 
temperature in natural systems. Our biofilm work suggests seasonal and depth-related changes in biofilm production and nutritional quality. Biofilms do form the base of food webs and future studies that have successfully manipulated temperature will benefit from examining the change in PUFA production in freshwater biofilms. 


\section{Chapter 2}

Measuring the Effects of Increased Water Temperature on the Production, Transfer, and Redistribution of Essential Fatty Acids in Freshwater Wetlands

\section{$\underline{2.1 \text { Introduction }}$}

The potential effects of climate change on several abiotic factors (e.g., temperature, water availability, carbon dioxide) simultaneously influence the fitness of organisms, both directly and indirectly, in all ecosystems (Parmesan \& Yohe 2003). Freshwater ecosystems are particularly vulnerable because they are typically small in size (Downing et al. 2006), yet disproportionally support high biodiversity (Dudgeon et al. 2006) and are heavily exploited by humans for various ecological goods and services (Woodward 2009). Thus, climate change, especially global warming, will affect freshwater ecosystems and the management of critical resources (Kundzewicz et al. 2008).

The GMST for 2081 - 2100, relative to 1986 - 2005, is expected to be 0.3 to $4.8^{\circ} \mathrm{C}$ warmer, depending on the rate of anthropogenic emissions (Collins et al. 2013). The predicted increase in GMST will increase water temperatures in lakes, streams/rivers, and wetlands worldwide as surface water temperature correlates strongly with air temperature (McCombie 1959; Morrill et al. 2005). Long-term records have confirmed accelerated warming in shallower waters of deep lakes, especially in summer months (Coats et al. 2006; Hampton et al. 2008). However, worldwide, freshwater systems are dominated by small $\left(<1 \mathrm{~km}^{2}\right)$ waterbodies (Downing et al. 2006), which typically respond more quickly to weather conditions than deeper water bodies (Gerten \& Adrian 2001) due to their higher surface-area-to-volume ratio.

In response to warming temperatures, aquatic ecosystems are expected to experience three main ecological responses: a) shift of species ranges from lower to higher latitudes (Hickling et al. 2006), b) seasonal shifts in life cycle events and trophic decoupling (Winder \& Schindler 2004), and, c) reduced body size (Daufresne et al. 2009). In addition, warming temperatures have recently also been predicted to reduce the production of n-3 LC-PUFA in marine and freshwater phytoplankton (Hixson \& Arts 2016).

Phytoplankton are the main producers of n-3 LC-PUFA, like eicosapentaenoic acid (EPA; 20:5n-3) and docosahexaenoic acid (DHA; 22:6n-3) in freshwater ecosystems (Hixson et 
al. 2015). Specific FA, or the overall composition, can be used to distinguish different taxa of phytoplankton (Lang et al. 2011; Taipale et al. 2013) as it has been suggested that phylogeny explains more variation in phytoplankton FA profiles than environmental factors (Galloway \& Winder 2015). In general, diatoms and cryptophytes produce high proportions of EPA while some chrysophytes and dinoflagellates produce high quantities of DHA (Lang et al. 2011; Taipale et al. 2013; Galloway \& Winder 2015). Chlorophytes and cyanobacteria, on the contrary, generally produce no or only trace quantities of EPA and/or DHA (Strandberg et al. 2015). The importance of EPA and DHA has been shown in the health of higher trophic levels including zooplankton (Gulati \& Demott 1997), fish (Bell et al. 1986), terrestrial predators (del Rio \& McWilliams 2016), and humans (Zárate et al. 2017), which explains the scientific community’s focus on these two particular PUFA. Thus, phytoplankton taxa play a key role in determining EPA and DHA content, however, temperature changes can also induce adaptive changes in the composition of FA in phytoplankton.

Homeoviscous adaptation is an essential biochemical adaptation for poikilothermic organisms (i.e., organisms that cannot control internal temperature) where the FA composition of cell membranes change in response to temperature to maintain fluidity (Sinensky 1974). The main characteristic of homeoviscous adaptation is that cell membranes become more fluid when exposed to higher levels of ambient kinetic energy and more solid when exposed to lower ambient levels of kinetic energy. The underlying basis for this is that temperature has a proportional relationship to kinetic energy and, therefore, can directly influence membrane fluidity. Low temperatures typically lead to the rigidification of membranes while high temperatures cause fluidization of membranes (Los \& Murata 2004). In response to cold temperatures, cells increase the proportion of PUFA in their membranes to increase fluidity and maintain proper membrane function (Hazel \& Williams 1990). In general, PUFA have lower melting points than SFA and can remain in a more disordered state at lower temperatures (Ernst et al. 2016). The increase of PUFA at lower temperatures has been observed in many aquatic organisms including phytoplankton (Nishida \& Murata 1996), invertebrates (Farkas et al. 1984), and fish (Brooks et al. 2002; Cossins et al. 2002). The increased membrane fluidity associated with high temperatures is compensated by cells incorporating SFA into their cell membranes, thus, reducing the proportion of PUFA (Hazel 1995; Oger \& Cario 2013). Sterols (e.g., 
cholesterol) can also play an important role in maintaining membrane fluidity (Crockett 1998) and increase at higher temperatures in phytoplankton (Ford \& Barber 1983; Piepho et al. 2012).

Early studies demonstrated changes in the ratio of PUFA to SFA in response to temperature changes, typically in marine phytoplankton (Ackman et al. 1968; Mortensen et al. 1988; Thompson et al. 1992b). Sushchik et al. (2003) demonstrated that both prokaryotic and eukaryotic phytoplankton respond to lower temperatures by producing higher proportions of PUFA. Similarly, Renaud et al. (2002) demonstrated a decrease in the proportion of EPA and DHA at higher temperatures in four tropical marine phytoplankton species. Teoh et al. (2004) did not find a decrease in the overall proportion of PUFA at higher temperatures in six Antarctic phytoplankton species, however, the one species that did produce EPA demonstrated lower proportions of that FA at higher temperatures. Pasquet et al. (2014) demonstrated the reduction of EPA and DHA at high temperatures in a marine diatom. However, some species (e.g., Emiliania huxleyi) have been shown to increase PUFA proportions, especially DHA, in response to warmer temperatures ( $\mathrm{Bi}$ et al. 2017, 2018), suggesting the possibility of a species-specific response to temperatures. Some studies have suggested that temperature may have a more drastic effect on reducing PUFA content in phytoplankton than $\mathrm{CO}_{2}$ concentrations (Bermudez et al. 2015), especially in PUFA-rich Antarctic species (Torstensson et al. 2013). Fuschino and colleagues (2011) demonstrated an increase in the proportion of SFA to PUFA in multiple lipid fractions of a common green algae, Scenedesmus obliquus. Specifically, the proportion of ALA (a precursor of EPA) declined in total lipid and glycerophospholipids (i.e., main components of biological membranes), demonstrating homeoviscous adaptation in the context of phytoplankton and climate warming (Fuschino et al. 2011). The effect of higher temperatures on phytoplankton PUFA has been well demonstrated in laboratory experiments, however, little work has been done to replicate laboratory studies by simulating climate warming in natural systems.

Controlled laboratory studies can reveal causal relationships between abiotics factors (e.g., higher temperatures) and biological responses (e.g., PUFA content), however, their limited realism inevitably simplifies a complex natural study system sometimes leading to spurious/erroneous conclusions. Mesocosms, experimental enclosures ranging from 1 to several thousand litres, capture a greater degree of biological complexity/realism and have been used as an essential tool in measuring community- and ecosystem-level responses to climate change (Stewart et al. 2013). 
Several studies have used mesocosms to study the effect of warming on freshwater lentic habitats, however, none have been conducted at a large scale (i.e., >10 L L; Stewart et al. 2013). Whole-system effects of temperature have been studied in the Hengill river basin in Iceland, a geothermal areas where underground heating (Arnason et al. 1969) produces a network of rivers with varying temperatures (O'Gorman et al. 2012), which represent a temperature gradient but cannot be manipulated to a specified temperature difference. At a smaller scale (i.e., $<10^{4} \mathrm{~L}$ ), freshwater lentic mesocosm studies have successfully manipulated temperature to mimic climate warming by using various submerged electrical heating elements (Liboriussen et al. 2005; Burnett et al. 2007; Yvon-Durocher et al. 2010; Greig et al. 2012), air-facilitated overhanging heating elements (Hines et al. 2013), open-top chambers (Strecker et al. 2004; Netten et al. 2008), greenhouse enclousre (Kankaala et al. 2000; Christensen et al. 2006) and hot water pipes (McKee et al. 2000; Baulch et al. 2003). These setups have been vital in understanding the potential effects of warmer temperature on freshwater lentic systems (Stewart et al. 2013). For example, the temperature-controlled (using hot water pipes) $3200 \mathrm{~L}$ open-air setup developed by McKee et al. (2000) was used to determine the effects of simulated climate change on water chemistry (McKee et al. 2003; Feuchtmayr et al. 2009) as well as communities of phytoplankton (Moss et al. 2003; Feuchtmayr et al. 2009), zooplankton (McKee et al. 2002a; Feuchtmayr et al. 2010), macroinvetebrates (Feuchtmayr et al. 2007), macrophytes (McKee et al. 2002b; Feuchtmayr et al. 2009), fish (Moran et al. 2010; Hopkins et al. 2011), and protists (Montagnes et al. 2002).

Conclusions from mesocosms experiments have, in some cases, been contradictory. For example, temperature was suggested to shift phytoplankton communities towards smaller species (Yvon-Durocher et al. 2011) while others found no effect on size (Moss et al. 2003).

Mesocosms, especially outdoor open-air types, are a good intermediate between laboratory and natural conditions, however, responses observed in controlled mesocosm experiments can be completely absent or opposite compared to responses observed in whole-lake or whole-pond systems (Graham \& Vinebrooke 2009). A major caveat associated with using smaller-scaled mesocosms is that they can differ in important ways from the ecosystem they are meant to represent. For example, nutrient concentrations can decline in mesocosms due to greater sedimentation rates and/or uptake by increased biofilm on mesocosm walls and typically require constant replenishment (Bloesch et al. 1988; Lennon et al. 2003). The stochastic nature of 
deploying in-situ mesocosm may not capture an accurate representation of natural biological community, however, all mesocosms have the potential to experience shifts in their biological community during the experiment (Jasser \& Kostrzewska-Szlakowska 2012; Dzialowski et al. 2014). Smaller-scaled mesocosm also have a high surface-area-to-volume ratio relative to natural systems, which can lead to "wall/edge effects" where, for example, organisms that favor edge habitats increase in biomass and ultimately, the mesocosm community diverges from a pelagic to more of a littoral-type community (Chen et al. 1997; Carpenter 1999; Petersen et al. 1999; Dzialowski et al. 2014). Temperature manipulation of a large whole-system has not been attempted and has been dubbed both "unfeasible" but also inevitably essential in understanding potential effects of climate warming (Stewart et al. 2013). In summary, mesocosms have been critical in understanding temperature-mediated responses and the underlying mechanisms, however, whole-lake/pond manipulations are required to capture realistic responses to higher temperatures, especially the production of PUFA.

The effect of increasing water temperature on phytoplankton biomass and community composition has been examined in both marine (e.g., Sommer \& Lengfellner 2008; Paul et al. 2015) and freshwater mesocosms (e.g., Moss et al. 2003; Feuchtmayr et al. 2009). In a freshwater context, field survey studies generally show a negative relationship between water temperature and zooplankton PUFA (Gladyshev et al. 2013), particularly DHA, content (Gladyshev et al. 2015) due, in large part, to the indirect effect of temperature on community composition (Gladyshev et al. 2011b). Laboratory experiments have demonstrated PUFA contents as well as the synthesis of PUFA in freshwater zooplankton to be lower at higher temperature (Masclaux et al. 2012). Marine mesocosms have been used to show both the negative (Garzke et al. 2016) and positive (Dahlgren et al. 2011) impacts of temperature on PUFA in zooplankton; however, the latter study was conducted at relatively lower temperature that may not induce PUFA-dominated membranes to switch to SFA-dominated membranes (i.e., 5 and $10^{\circ} \mathrm{C}$ ). Production of DHA in freshwater invertebrates was higher in cold-water sections than warm-water sections of a thermally-gradient freshwater river (Kalachova et al. 2013), again due to changes in community composition (Gladyshev et al. 2016).

Recently, scientific interest has peaked with respect to studies that examine the coupling of aquatic to terrestrial ecosystems (Schulz et al. 2015), and specifically in regards to the transfer of essential nutrients from freshwater to terrestrial ecosystems (e.g., Borisova et al. 2016; 
Martin-Creuzburg et al. 2017). Amphibiotic insects (i.e., insects that have aquatic larval stages but have a terrestrial adult stage) are important vectors of EFA across the aquatic-terrestrial boundary. In particular, the emergence of species from the Chironomidae family (the Diptera order) has been quantified, both in biomass and PUFA content. The larval forms of different species have been shown to occupy different ecological niches in a lake and thus assess to nutritionally-different prey, which explain some differences observed in larval PUFA content (Makhutova et al. 2017). Species of Chironomidae have been shown to contribute the most to PUFA export (due to high emergence biomass) in a mid-sized mesotrophic lake (MartinCreuzburg et al. 2017) and estimated to export $1.752 \mathrm{mg}$ PUFA $\cdot \mathrm{m}^{-2} \cdot \mathrm{y}^{-1}$ in a slightly smaller oligotrophic mountain lake (Borisova et al. 2016). The average deposition of PUFA by emerging insects onto terrestrial environments (within $100 \mathrm{~m}$ from shore) has been estimated at $150 \mathrm{mg}$. $\mathrm{m}^{-2} \cdot \mathrm{y}^{-1}$ (Martin-Creuzburg et al. 2017), representing a substantial source of EFA to riparian predators, higher than previous estimates for EPA and DHA (Gladyshev et al. 2009). In addition, feeding experiments have clearly demonstrated the importance of these FA subsidies to terrestrial predators such as birds (Twining et al. 2016) and spiders (Fritz et al. 2017). Therefore, the potential negative effect of warming temperatures on the PUFA content in larval stages of invertebrates may lead to a reduced availability of these essential nutrients to both higher aquatic trophic levels as well as riparian predators.

In this chapter, I describe an in-situ temperature manipulation experiment of a freshwater pond purposefully designed to examine the effects of higher water temperature on PUFA, a) production in phytoplankton and biofilm, b) transfer to zooplankton and insect larvae, and, c) redistribution to adjacent terrestrial ecosystem via insect emergence. Smaller mesocosm studies have used underwater pipes (e.g., McKee et al. 2000) and successfully manipulated temperature. I employed an underwater heating system made of metal and plastics pipes to deliver heat to a large mesocosm ( 300,000 L) within a freshwater pond. Vertical hauls, in several locations within a treatment, with a water sampler and plankton net captured the overall phytoplankton and zooplankton community. Ceramic tiles incubated on a submerged incubator platform were used to collect aquatic biofilm. Finally, insect larvae were collected from the sediment using an invertebrate sampler (i.e., D-net) and adult insects were collected with a floating insect emergence trap. In an attempt to capture other physiological and ecosystem responses to warming, supplementary objectives were also attempted. In order to quantify the effect of a 
warming world on mercury dynamics within a food web and a freshwater system, most biological samples (mentioned above), along with water and sediment samples, were collected for mercury analysis. Additionally, the potential effects of warmer temperature on Daphnia metabolomics (i.e., complete profile of metabolites), using both in-situ and ex-situ methods was examined by incubating a lab-reared (from a single clonal female) Daphnia magna in submerged cages.

Based on previous literature, I predicted that the production of PUFA in phytoplankton and biofilm would be reduced in the warm treatment as microorganisms typically reduce the proportions of PUFA in their cell membranes in response to warmer temperatures. Zooplankton PUFA content should therefore theoretically decrease, however, bioconversion of C18-PUFA precursors may mask temperature-mediated effects. Finally, the PUFA contents in aquatic larvae and their adult counterparts were both predicted to be lower. In all cases where PUFA content is predicted to decrease, an increase in SFA, especially at the base of the food web (i.e., phytoplankton and biofilm), would suggest homeoviscous adaptation as a possible response mechanism to warming temperatures. Results from such a large-scaled manipulation experiment would provide a more realistic assessment of biochemical responses of freshwater communities to the predicted increase in water temperature as a result of climate warming. 


\section{$\underline{2.2 \text { Methods }}$}

In order to assess the effect of warmer temperatures on PUFA production, transfer, and re-distribution in freshwater systems, an in-situ manipulation experiment was conducted. A candidate pond in Whitby, Ontario, Canada was selected in fall 2015 and depth measurements were taken in December 2015. Measurements were used to design and subsequently build thermally-insulating curtains to divide the selected pond into sections. An indirect warming system was developed and built specifically for the selected pond. Curtains and warming system were deployed in May 2017 and warming (checked via portable temperature loggers) was attempted until July 2017. No specific permits were required for the described experiment. Experiment was performed on privately-owned land with prior consent from the landowners (Peter and Elaine Leigh).

\subsubsection{Pond Selection}

To screen for a candidate pond within the Greater Toronto Area (Fig. 2.1), a set of general characteristics, along with Google Earth ${ }^{\mathrm{TM}}$, were used. Possible candidate ponds were selected if: a) their surface area was $<3 \mathrm{~km}^{2}$, b) they had no visible surface water connections, c) there was an established, or at least a potential, supply of electrical power nearby. Within the search area, $>500$ potential ponds were located. In-person field surveys of ponds further narrowed potential ponds that, a) were naturally-formed or a man-made with sufficient undisturbed time (>10 years) for natural communities to become established, b) were not within a $1 \mathrm{~km}$ radius or immediately "downhill" of an active farming operation, and c) had a confirmed electrical power supply.

From a few final candidate ponds ( $<10$ ponds), a medium-size mesotrophic freshwater pond in Whitby, Ontario (435 59' 58.1892" N, 79² $2^{\prime} 9.9384^{\prime \prime} \mathrm{W}$ ) was selected for the in-situ manipulation. The pond is $\sim 100 \mathrm{~m}$ long and $\sim 17 \mathrm{~m}$ wide $\left(2,121 \mathrm{~m}^{2}\right)$ with an average and maximum depth of $2.92 \mathrm{~m}$ and $5.1 \mathrm{~m}$, respectively. Depth measurements were taken in December 2015 using a sonar depth finder (Cole-Parmer®; Portable Water Depth Sounder Gauge; RK-99756-40). The pond was divided along a $2 \mathrm{~m} \times 2 \mathrm{~m}$ grid and measurements were taken at all points for most of the pond, after which measurements were taken $\sim 2 \mathrm{~m}$ along the center line for the entire length of the pond. 


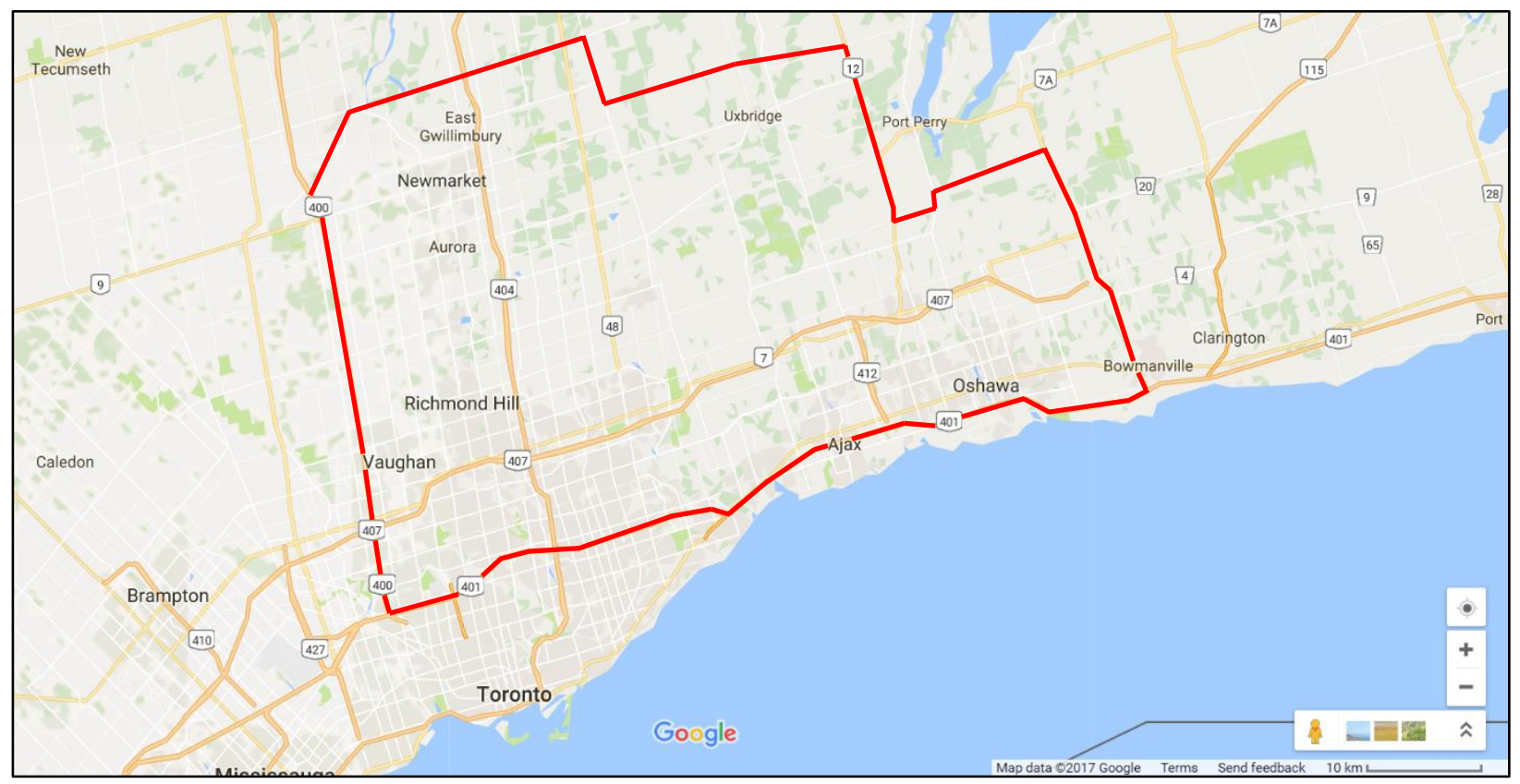

Figure 2.1: Search area for our candidate pond in Greater Toronto Area. The red line highlights the boundaries of the search area, $\sim 2,500 \mathrm{~km}^{2}$. Image captured on Google Maps.

\subsubsection{Curtain and Heating System}

The selected pond was too large to attempt a whole-system manipulation; therefore, I attempted to conduct the experiment on a portion of the pond. A portion of the pond (i.e., in-situ mesocosms) was divided so as to represent ambient, control, and experimental conditions (Fig. 2.2). The ambient area was chosen to account for effects of the curtains and heating system. I attempted to heat the water in the experimental area to $4^{\circ} \mathrm{C}$ above the temperature of the control area to simulate predicted temperatures (as a result of climate warming) for temperate latitudes by the end of the $21^{\text {st }}$ century (Collins et al. 2013).

The pond curtains that divided the pond into sections were built from polyethylenecoated tarps (Uline®; Standard Woven Poly Tarps; S-8401) and 0.635-cm thick polyethylene foam (Uline®; Non-Perforated Foam Rolls; S-1855). The pond curtain was composed of 0.635cm thick layer of polyethylene foam "sandwiched" between two layers of polyethylene tarp, held together with 20-cm black UV-resistant cable zip ties (Commercial Electric®; 8-inch UV Cable Tie; GT-200STB). The two outside pieces of tarp acted as a physical barrier to prevent water exchange between the sections while the foam within provided some insulation to prevent heat transfer from the experimental section. The pond curtains were made based on the curvature of 
the pond (determined by depth measurements), however, an extra $0.6 \mathrm{~m}$ length of curtain was added to the total curtain depth to account for possible increases in water level during the season (e.g., due to a flood event). To divide the control and experimental area from the ambient area, a curtain was deployed $\sim 30 \mathrm{~m}$ from the West shore (referred herein as shorter curtain), at the narrowest point of the pond (see vertical curtain in Fig. 2.2). The control and experimental area were divided into approximately two equal sections (based on volume calculated from depth measurements) by two curtains (referred herein as longer curtains) with approximately $1 \mathrm{~m}$ of water in-between to minimize heat transfer from the experimental section (see two horizontal curtains in Fig. 2.2).

An electric pool heater (Consolidated Pool and Spa Industries®; K-Star 5KW Electric Pool/Spa Heater; K-5-D) and pool pump (Hayward®; MaxFlo ${ }^{\text {TM }} 0.75$ HP Pool Pump; SP2805X7) were used to attempt to raise the water temperature of the experimental area. An underground electrical wire was installed by a licensed electrician to supply power (240 V, $30 \mathrm{~A})$ from the nearby residence to a subpanel near the edge of the pond, where the pool heater and pump were installed. Hot water generated by the pool heater was pumped through an underwater piping system at the bottom of the pond, designed to transfer heat conductively to the water column. The underwater piping system (see white and black lines in Fig. 2.2) was built using rigid PVC ( $\sim 5 \mathrm{~cm}$ by $\sim 3 \mathrm{~m}$, Schedule 40$)$ and aluminum pipes ( $\sim \mathrm{cm}$ by $1.5 \mathrm{~m}$, Schedule 40$)$, where most of the heat transfer was expected around the aluminum pipes spread out through the experimental area. The PVC pipes were primed (Oatey®; Purple Primer; 30757) and chemically bond (Oatey®; PVC Cement Rain-R-Shine; 31582) to PVC adapters ( 5-cm PVC Schedule 40 Female Slip x Male NPT). All aluminum pipes were machine-threaded (Male NPT) on both ends. Metal and PVC pipe threads were then taped with thread seal tape (Oatey® PTFE Pink Thread Seal Tape; 31402D) and coated with thread sealant paste (Oatey®; Great White® Pipe Joint Compound with PTFE; 31233$)$ prior to being screwed into stainless steel adapter $(\sim 5-\mathrm{cm}$ Schedule 40 Female NPT x Female NPT) and tightened by hand using pipe wrenches. The configuration of plastic male threads with female metal adaptors provided leak-proof connections between PVC and aluminum pipes for high temperatures and water pressure. 


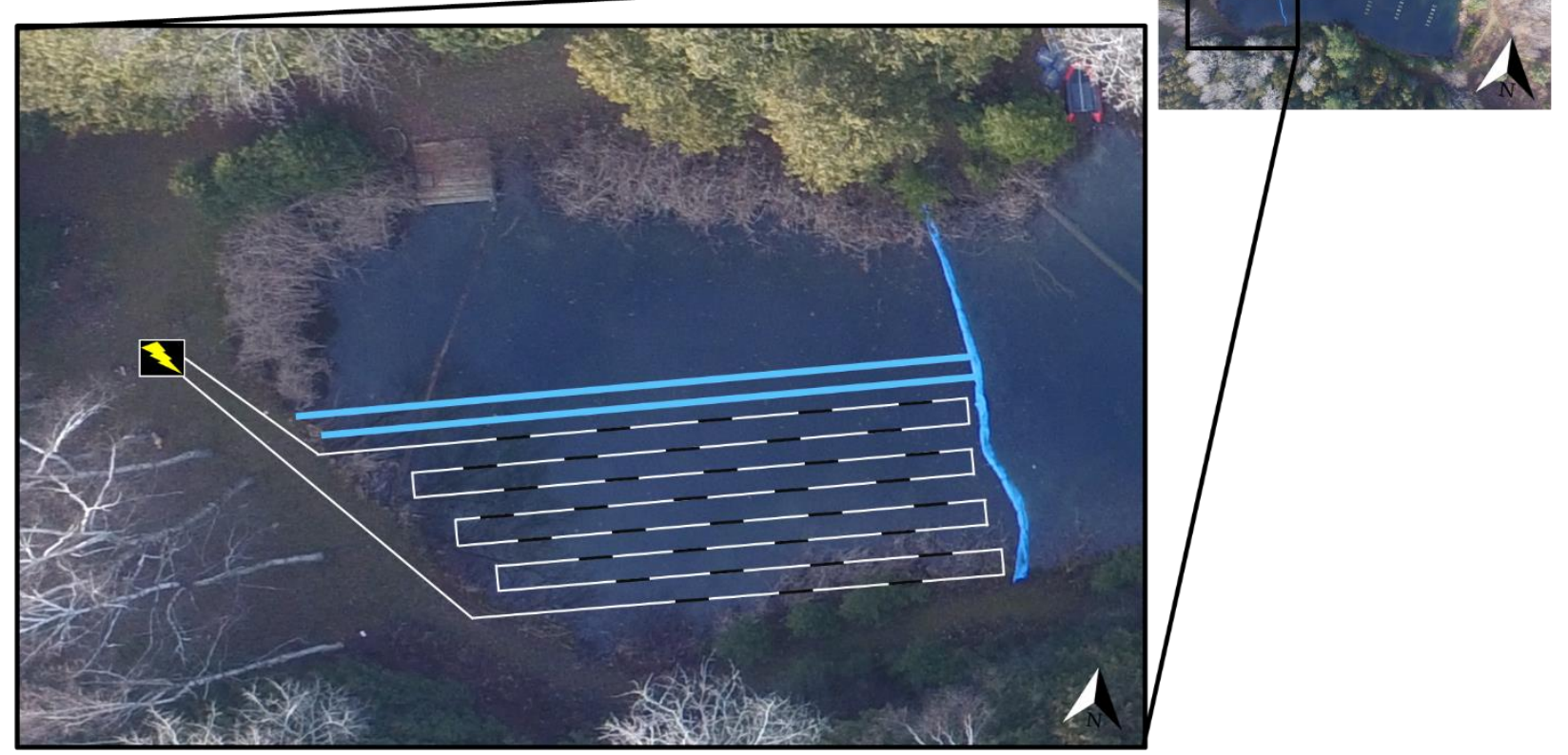

Figure 2.2: Pond sections and schematic design of experimental setup. The curtain (blue lines) divided the section into control (top), experimental (bottom), and ambient (right). Pool heater and pump were installed near the pond (black box with lightning bolt) connected to a radiatorstyle heating system composed of alternating PVC (white lines) and aluminum (black lines) pipes.

\subsubsection{Installation}

In May 2017, the pond curtains and underwater piping systems were deployed. The shorter curtain was deployed first to separate the control and experimental sections from the ambient area. Pool noodles were attached to the top of the curtains to prevent the curtain from sinking and then the curtains were floated into position. To sink the curtain to the sediment, $\sim 11$ $\mathrm{kg}$ of sand was added to polypropylene sandbags and attached, at tightly-spaced intervals (every $\sim 30-40 \mathrm{~cm}$ ) to the bottom of the curtain by folding the sandbag into the curtain and securing it using several cable ties. The underwater piping system was then floated out into position in the experimental mesocosm. PVC sewer pipes ( 10-cm diameter and $\sim 50$-cm height; IPEX HomeRite Products®; Solid Sewer Pipe; 004043) with a glued cap (together referred herein as PVC legs) were loosely attached around metal pipes to lift the piping system off the bottom of the pond to prevent direct heating of the sediment. The underwater piping system was then filled with residential water and sunk into position. Then both longer curtains that separate the control and experimental area were deployed, exactly as described with the shorter curtain. Two scuba 
divers attached both longer curtains to the shorter curtain using rope, ensured the curtain was sunk into the sediment with the sandbags, added sandbags to close large gaps, and ensured that the PVC legs were horizontal and lifting the piping system off the sediment.

\subsubsection{Start of Experiment and Temperature Measurements}

The temperature of the pond was monitored hourly using several vertical (i.e., within the water column) and horizontal (i.e., on the sediment) thermistor chains (Fig. 2.3). I built several vertically-deployed thermistor chains equipped with submersible temperature data loggers (Alpha Mach®; iBWetLand; AM015) that were contained within $50 \mathrm{~mL}$ Falcon tubes that were attached to a rope at set distances. Several holes were drilled into the Falcon tubes to allow water within the Falcon tube to thermally equilibrate with water outside, minimizing the creation of a microenvironment (J. Chételat, Environment Canada and Climate Change, pers. comm.). Vertical thermistor chains were attached to an anchor (i.e., brick) at one end and a buoy (i.e., pool noodle) at the other end to prevent slack in the rope. Vertical chains were deployed in three locations in the control and experimental areas and one location in ambient area (see colored squares in Fig. 2.3). Data loggers were attached every $1 / 2$ meter from the anchor (i.e., bottom of pond) and the depth of the data logger nearest to the surface was adjusted to be $25 \mathrm{~cm}$ from the surface.

In addition, horizontal thermistor chains were attached to the curtain and data loggers were attached every meter ( $2 \mathrm{~m}$ in the ambient area) up until the shore. Two horizontal chains were deployed in both the control and experimental areas, one was deployed attached to the shorter curtain on the experimental side (see black lines in Fig. 2.3). All temperature measurements were taken every hour at $0.06^{\circ} \mathrm{C}$ resolution. Thermistor chain measurements were not readily accessible, therefore, occasional "real-time" sampling of temperature was done by lowering a YSI ${ }^{\mathrm{TM}}$ Model $600 \mathrm{QS}$ near thermistor chain locations. YSI temperature measurements were taken at noon to prevent canopy cover affecting temperature readings. The pool heater and pump were turned on June $5^{\text {th }}, 2017$ (official experiment start) and terminated June $28^{\text {th }}, 2017$. 


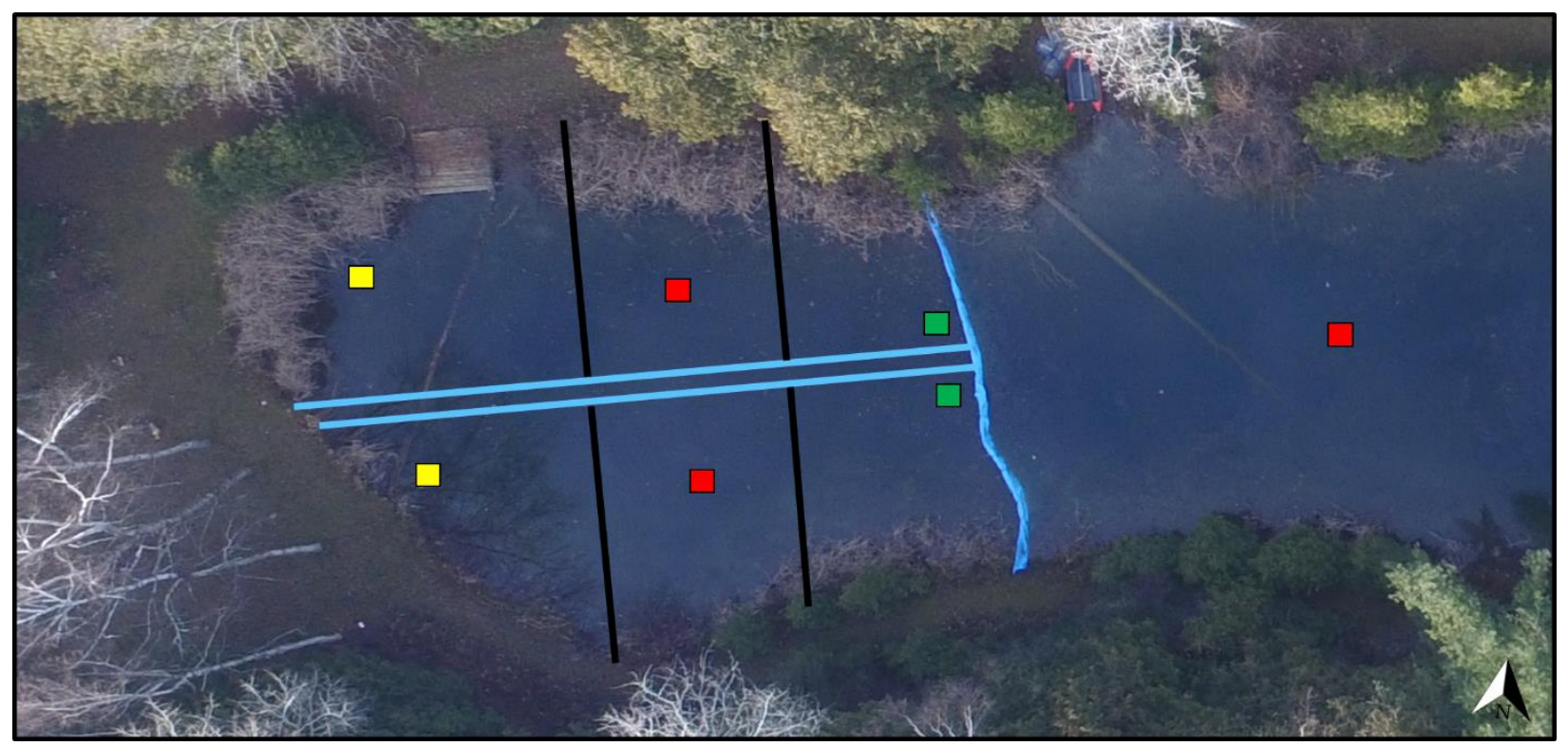

Figure 2.3: Location of temperature probes during experiment. Vertical thermistor chains were deployed in three locations: corner (green box), middle (red box), and shallow (yellow box) to represent different depths. Black lines represent the horizontal thermistor chains that were deployed along the sediment in east (right) and west (left) locations.

\subsubsection{Sample Collection}

Samples were collected from all three treatments (i.e., ambient, control, and experimental), unless otherwise specified. Water samples were collected using an integrated water sampler, which was a depth-marked PVC pipe ( 5-cm diameter) attached to a one-way check valve with a flexible pipe coupling. Pond water was pre-filtered through $51-\mu \mathrm{m}$ and $28-\mu \mathrm{m}$ mesh prior to being vacuum-filtered through a $0.2-\mu \mathrm{m}$ polyethersulfone membranes filter (VWR ${ }^{\text {TM; }}$ Supor ${ }^{\circledR}$ Membrane Disc Filters; CA28147-978). Filtered pond water (250 ml) was collected in Milli-Q rinsed Nalgene ${ }^{\circledR}$ PETG bottles (VWR ${ }^{\mathrm{TM}}$; 16059-740) and stored at $-20^{\circ} \mathrm{C}$ until nutrient (phosphorus and nitrogen species) and metal (specifically, aluminum) analysis.

Phytoplankton were sampled weekly using the same integrated water sampler. Collected water was pre-filtered through a 51- $\mu \mathrm{m}$ mesh to remove debris and larger animals. To collect sample for FA, sterols, chl $a$, and particulate organic carbon (POC) analysis, $500 \mathrm{~mL}$ of meshfiltered water was vacuum filtered $(<0.5 \mathrm{psi})$ onto triplicate pre-combusted $\mathrm{GF} / \mathrm{F}$ filters (Whatman®; pre-combusted at $450^{\circ} \mathrm{C}$ for $4 \mathrm{~h}$ ). For phytoplankton identification, $25 \mathrm{~mL}$ of the pre-filtered pond water was preserved in Lugol's solution $(1 \% \mathrm{v} / \mathrm{v})$ in a glass vial, which was covered in aluminum foil and stored in a cool and dark place for microscopic counting. Zooplankton were also sampled weekly by repeated vertical hauls using a $150-\mu \mathrm{m}$ plankton net. 
Using a stereoscope, zooplankton were picked and partitioned based on broad taxa (i.e., cladocerans and copepods) into pre-weighed cryovials for FA analysis. Multiple vertical hauls for water, phytoplankton, and zooplankton samples were combined from three locations to collect a representative sample of the treatment. Insect larvae and other macroinvertebrates were sampled by kick-netting with a D-frame dip net (600 $\mu \mathrm{m}$; Wildco ${ }^{\circledR}$; 3-425-D63) along within a one-metre radius for five-minute intervals. The collected organisms were then picked and grouped by taxa, then placed in pre-weighed cryovials for FA analysis. To collect biofilm, unglazed ceramic tiles were incubated on a platform (discussed in detail in Methods of Chapter 3) suspended $25 \mathrm{~cm}$ from the water surface. Platforms were deployed in a central location within the treatment to minimize differences in underwater light climate.

Adult insects were collected using floating emergence traps, which were equally placed between treatments and two water depths $(80$ and $150 \mathrm{~cm})$. The traps were composed of fine mosquito netting mesh overtop a square-pyramid PVC frame supported by pool noodles that kept the trap afloat (Fig. 2.4). Emerging insects were captured into the removable plastic bottles connected to the trap. Insects were collected from the bottle and trap using an insect aspirator, stored at $-20^{\circ} \mathrm{C}$ to limit movement, grouped by taxa, and then stored in cryovials for FA analysis. All samples for FA analysis were immediately placed at $-20^{\circ} \mathrm{C}$ (in the freezer at the landowner's residence) but were transferred on ice (vehicle transport, within $24 \mathrm{~h}$, back to lab) and stored at $80^{\circ} \mathrm{C}$ until analysis.

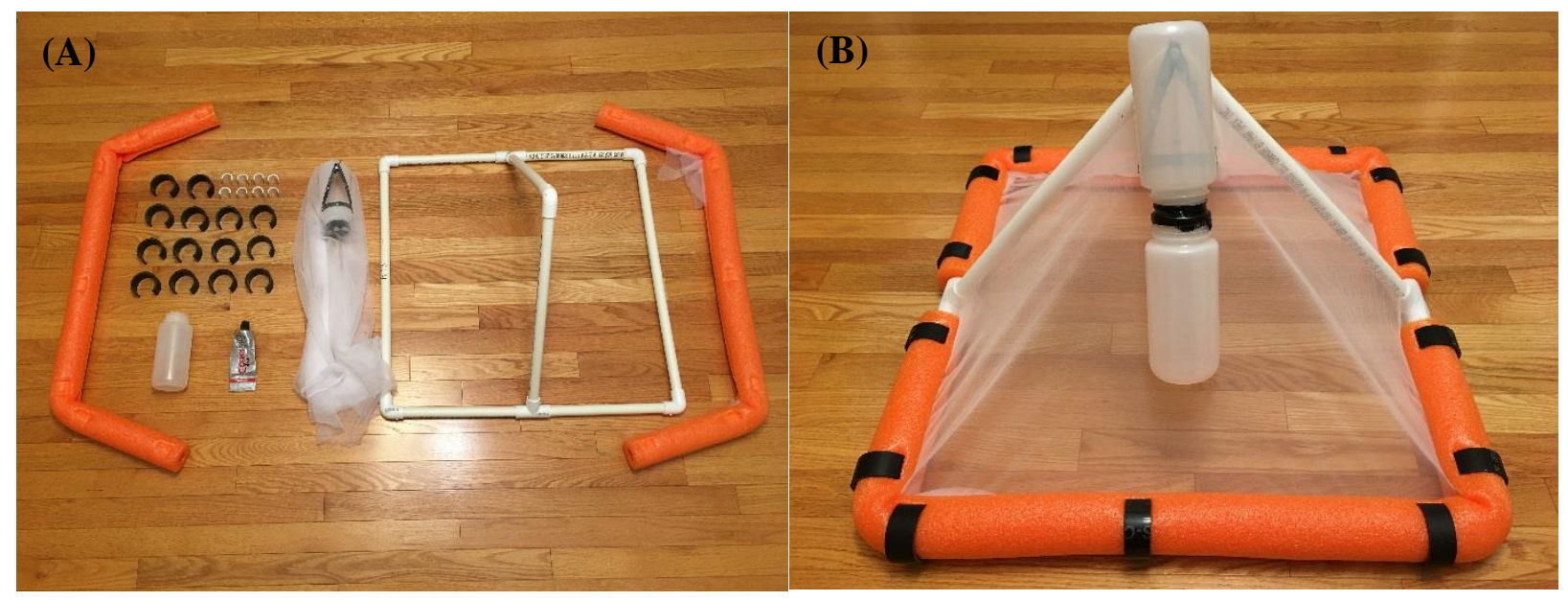

Figure 2.4: Design of floating insect emergence traps. All the components of the traps (A) are constructed to a (B) fully-built aquatic insect emergence trap. Traps were built using the design suggested by Cadmus et al. (2016). 
Water samples were collected monthly for mercury analysis using clean-hands dirtyhands technique outlined by the U.S. Environmental Protection Agency protocol (EPA 1996) and collected in Nalgene ${ }^{\circledR}$ PETG bottles (VWR ${ }^{\mathrm{TM}}$ 16059-740). Samples were acidified to $1 \%$ using trace metal-grade $\mathrm{HCl}$ (VWR ${ }^{\mathrm{TM}}$; OmniTrace Ultra ${ }^{\mathrm{TM}}$; CAHX0608) within $24 \mathrm{~h}$ and stored at $4^{\circ} \mathrm{C}$. Sediment samples for mercury analysis were not collected due to the hard-bottom nature of the pond as core samplers were unable to penetrate the sediment.

Pre-experiment zooplankton hauls suggested Daphnia species were absent in the pond. Therefore, to examine Daphnia metabolomics, a clone line of D. magna females was cultured (to control for genetic differences) at Dorset Environmental Science Centre in Hard Combo (Baer and Goulden 1998; Kilham et al. 1998), a hard-water media (Appendix 2) for freshwater algae and zooplankton. To prevent the introduction of a zooplankton species into the pond, in-situ cages were built to incubate Daphnia prior to metabolomics analysis. Briefly, the bottom of a $1.18 \mathrm{~L}$ plastic container was cut out and both the top and bottom were covered and sealed with $200-\mu \mathrm{m}$ Nitex ${ }^{\circledR}$ mesh, which allowed phytoplankton to flow through the cages but prevented the Daphnia from escaping. The cages were suspended horizontally $50 \mathrm{~cm}$ from the surface, such that the top and bottom of container were perpendicular to the sediment. Two cages were deployed in both the control and experimental section with $~ 50 \mathrm{D}$. magna individuals in each cage. The cages were left untouched for $10 \mathrm{~d}$, after which the Daphnia were removed from the cages and placed in $1 \mathrm{~L}$ mason jars with 51- $\mu \mathrm{m}$ filtered pond water for analysis. Live specimens could be used for in-vivo metabolomics while lyophilized samples were used for ex-vivo analysis via nuclear magnetic resonance (NMR) and mass spectrometry (MS). 


\section{$\underline{2.3 \text { Results }}$}

\subsubsection{YSI Temperature Measurements}

Real-time temperature measurements (via YSI) were taken on June $11^{\text {th }}$ and June $20^{\text {th }}$ to monitor the temperature of the middle area of all three sections of the pond. Measurements taken on June $11^{\text {th }}$ demonstrated a $\sim 4.5^{\circ} \mathrm{C}$ increase in water temperature in the experimental section compared to the ambient section at $3 \mathrm{~m}$ deep (Fig. 2.5). Similarly, water temperature in the experimental section was $\sim 4^{\circ} \mathrm{C}$ warmer than the control side at $2.5 \mathrm{~m}$ deep (Fig. 2.5). On June $20^{\text {th }}$, the experimental section was $\sim 2.5^{\circ} \mathrm{C}$ and $1.75^{\circ} \mathrm{C}$ warmer than control (at $2.4 \mathrm{~m}$ ) and ambient side (at $2.5 \mathrm{~m}$ ), respectively (Fig. 2.6).

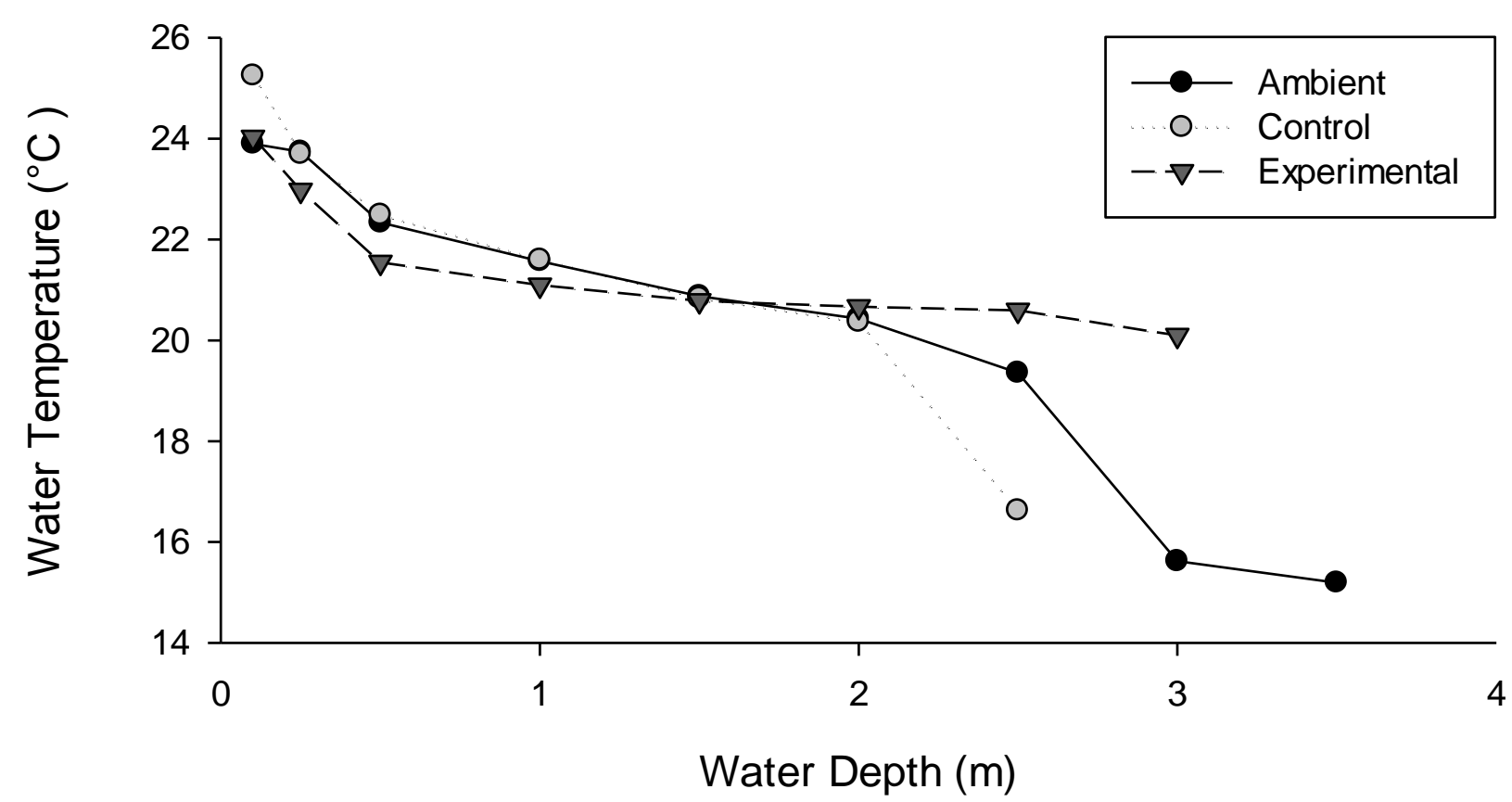

Figure 2.5: Temperature measurements (via YSI) of three pond sections on June $11^{\text {th }}$ at varying depths. All measurements were taken within an hour of noon in the middle of each section. 


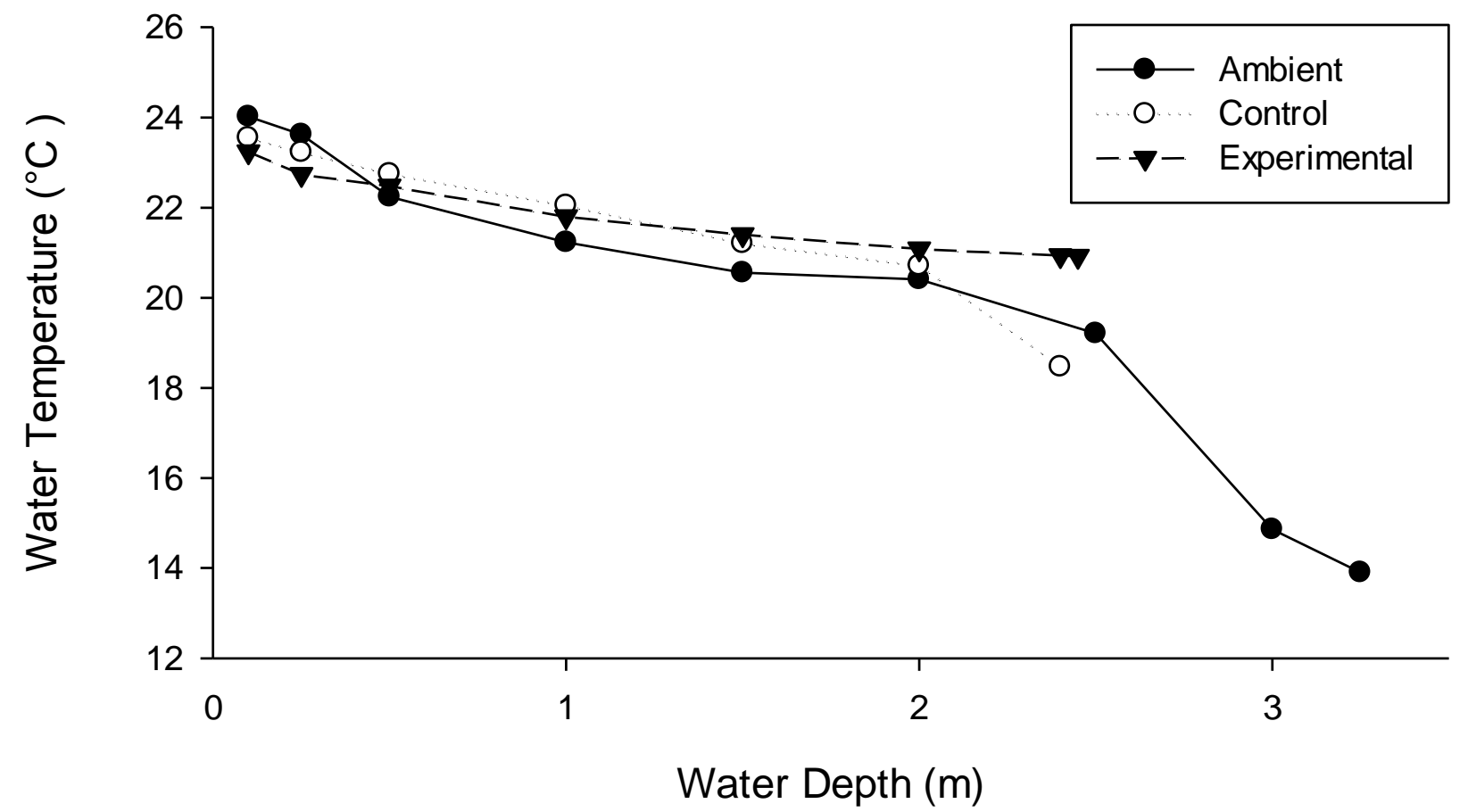

Figure 2.6: Temperature measurements (via YSI) of three pond sections on June $20^{\text {th }}$ at varying depths. All measurements were taken within an hour of noon in the middle of each section.

\subsubsection{Vertical Thermistor Chain Temperature Measurements}

Vertical thermistor chains were extracted on June $21^{\text {st }}$ and June $28^{\text {th }}$, at which time data from iButtons was downloaded and analyzed; only data from June $28^{\text {th }}$ will be presented for comparison between corner, middle, and shallow locations (Table 2.1). In the corner location (i.e., deepest points of both ponds), warming of the experimental side did occur $17 \mathrm{~d}$ into the experiment (Fig. 2.7A). At $0.5 \mathrm{~m}$ from the bottom, the experimental corner was on average $0.7^{\circ} \mathrm{C}$ (maximum $1.2^{\circ} \mathrm{C}$ ) warmer from Julian Day 172 until the end of the experiment. At all other depths, warming did not occur, and little to no temperature differences were seen between the control and experimental corner (Fig. 2.7B-E). From Julian Date 158 to 164, the control corner 2 meters from the bottom was on average $0.5^{\circ} \mathrm{C}$ (maximum $0.7^{\circ} \mathrm{C}$ ) warmer than the experimental (Fig. 2.7D). In the middle section, temperature varied more between depths than treatments (Fig. 2.8). Through the entire experiment, at $0.5 \mathrm{~m}$ from the bottom the control and experimental middle was on average $2.7^{\circ} \mathrm{C}\left(\max .4 .1^{\circ} \mathrm{C}\right)$ and $2.9^{\circ} \mathrm{C}\left(\max .4 .1^{\circ} \mathrm{C}\right)$ warmer than the ambient middle, respectively (Fig. 2.8A). This trend was also observed at 1.0 and $1.5 \mathrm{~m}$ from the bottom but for shorter periods of time (Fig. 2.7B and C). At $0.25 \mathrm{~m}$ from the surface, the experimental 
middle was regularly cooler than both the control and ambient middle (Fig. 2.8D). In the shallow region, both the control and experimental temperature were similar (Fig. 2.9).

Table 2.1: Water temperature data $\left({ }^{\circ} \mathrm{C}\right)$ of vertical thermistor chains of all sections in all locations at all depths measured. Values are presented as mean $\pm \operatorname{SD}(n)$.

\begin{tabular}{lccc}
\hline \multicolumn{1}{c}{ Location/Depth } & Ambient & Control & Experimental \\
\hline \hline Corner Spot & & $20.0 \pm 1.3(549)$ & $20.3 \pm 1.4(549)$ \\
$0.5 \mathrm{~m}$ from bottom & & $21.1 \pm 1.6(549)$ & $21.1 \pm 1.6(549)$ \\
$1.0 \mathrm{~m}$ from bottom & & $21.4 \pm 1.7(549)$ & $21.3 \pm 1.7(549)$ \\
$1.5 \mathrm{~m}$ from bottom & & $21.5 \pm 1.7(549)$ & $21.3 \pm 1.7(549)$ \\
$2.0 \mathrm{~m}$ from bottom & & & $22.1 \pm 1.7(533)$ \\
$0.25 \mathrm{~m}$ from surface & & & \\
& & $21.0 \pm 1.6(534)$ & $21.2 \pm 1.6(534)$ \\
Middle Spot & $18.3 \pm 0.8(534)$ & $21.3 \pm 1.6(534)$ & $21.3 \pm 1.6(534)$ \\
$0.5 \mathrm{~m}$ from bottom & $21.0 \pm 1.4(534)$ & $21.6 \pm 1.6(534)$ & $21.6 \pm 1.6(534)$ \\
$1.0 \mathrm{~m}$ from bottom & $21.4 \pm 1.5(534)$ & $22.1 \pm 1.7(534)$ & $21.9 \pm 1.6(534)$ \\
$1.5 \mathrm{~m}$ from bottom & $22.4 \pm 1.7(534)$ & & \\
$0.25 \mathrm{~m}$ from surface & & $22.4 \pm 1.0(403)$ & $22.3 \pm 0.9(403)$ \\
& & $22.6 \pm 1.1(403)$ & $22.4 \pm 1.0(403)$ \\
Shallow Spot & & & \\
$0.5 \mathrm{~m}$ from bottom & & & \\
$0.25 \mathrm{~m}$ from surface & & & \\
\hline
\end{tabular}




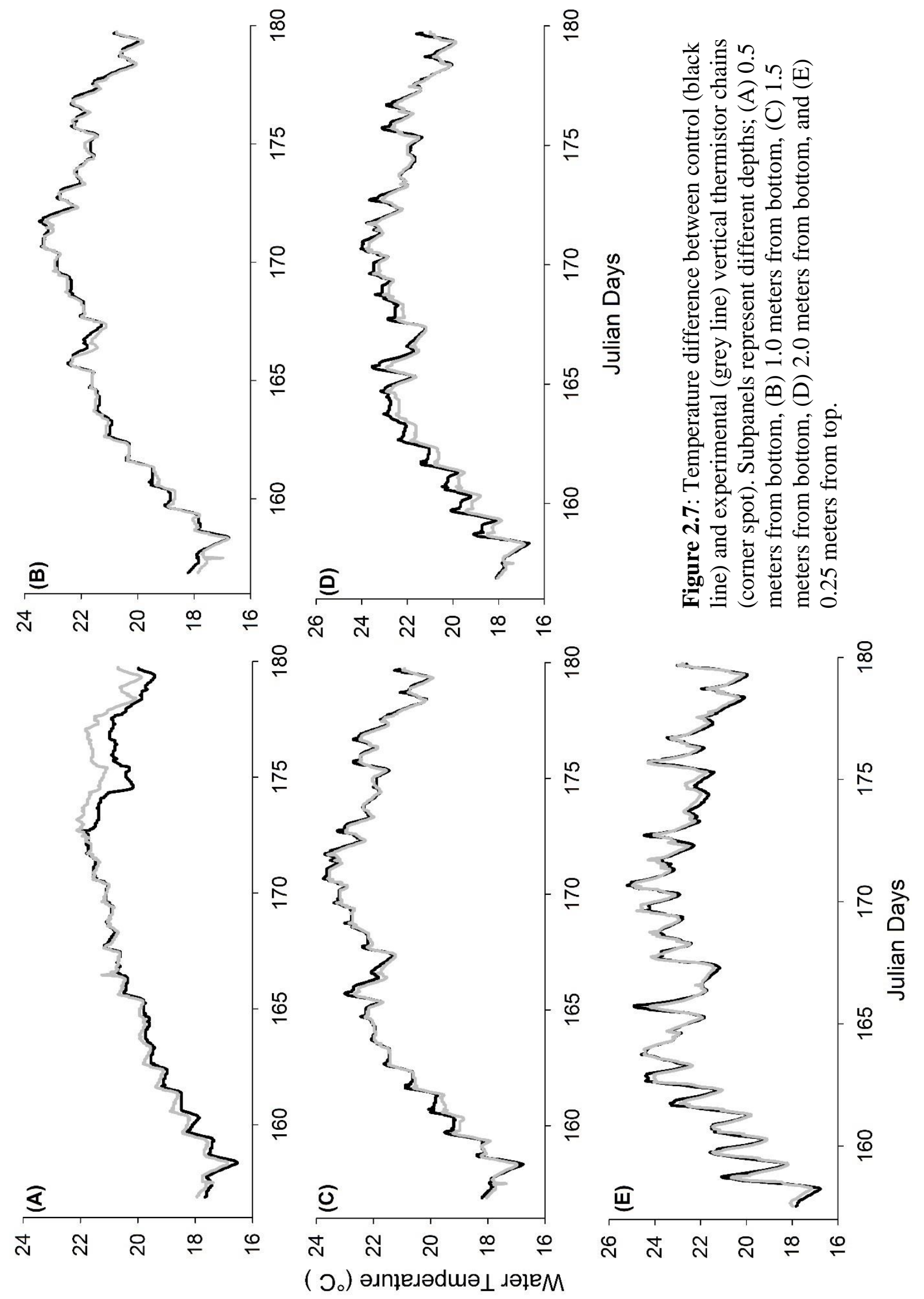




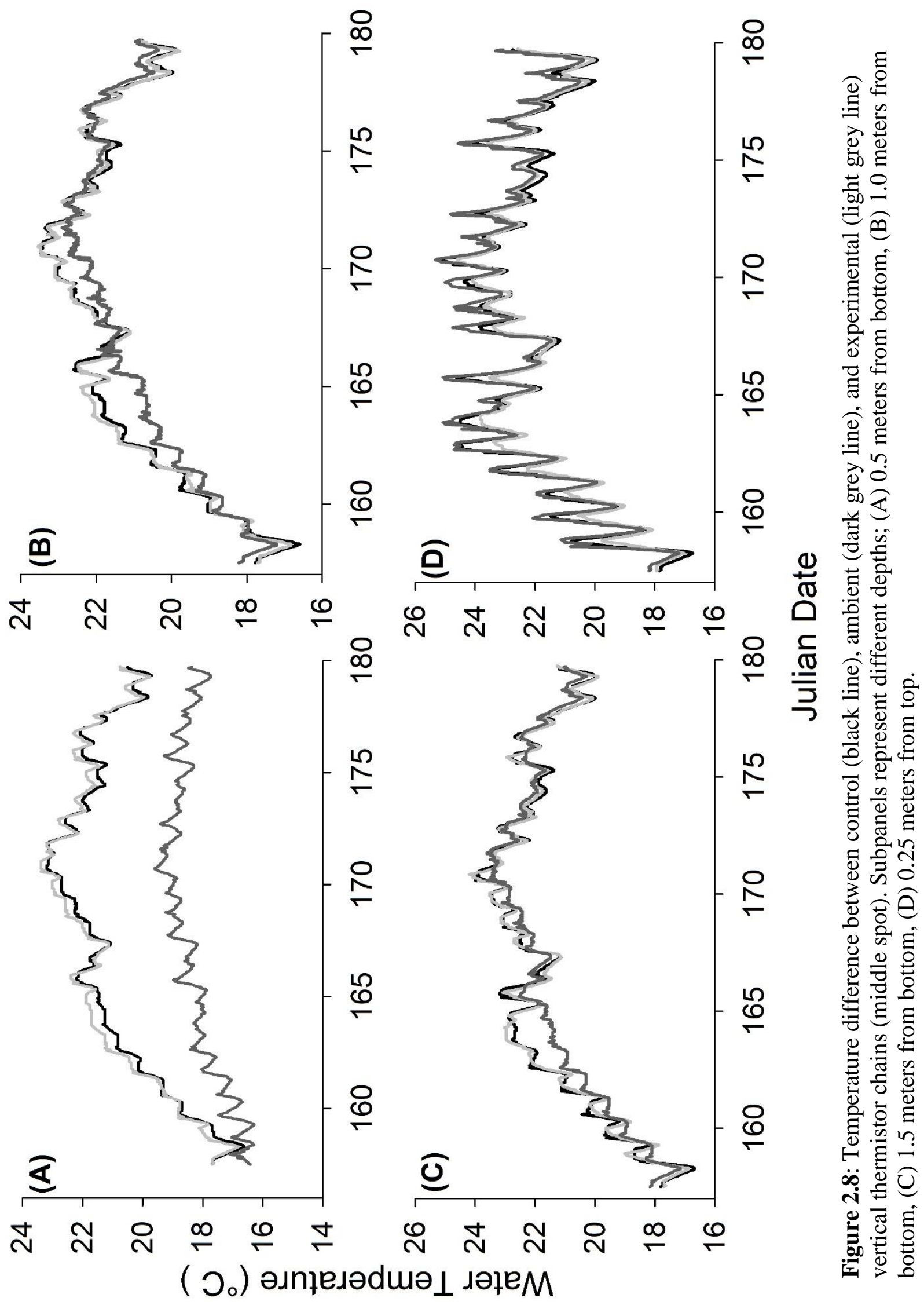




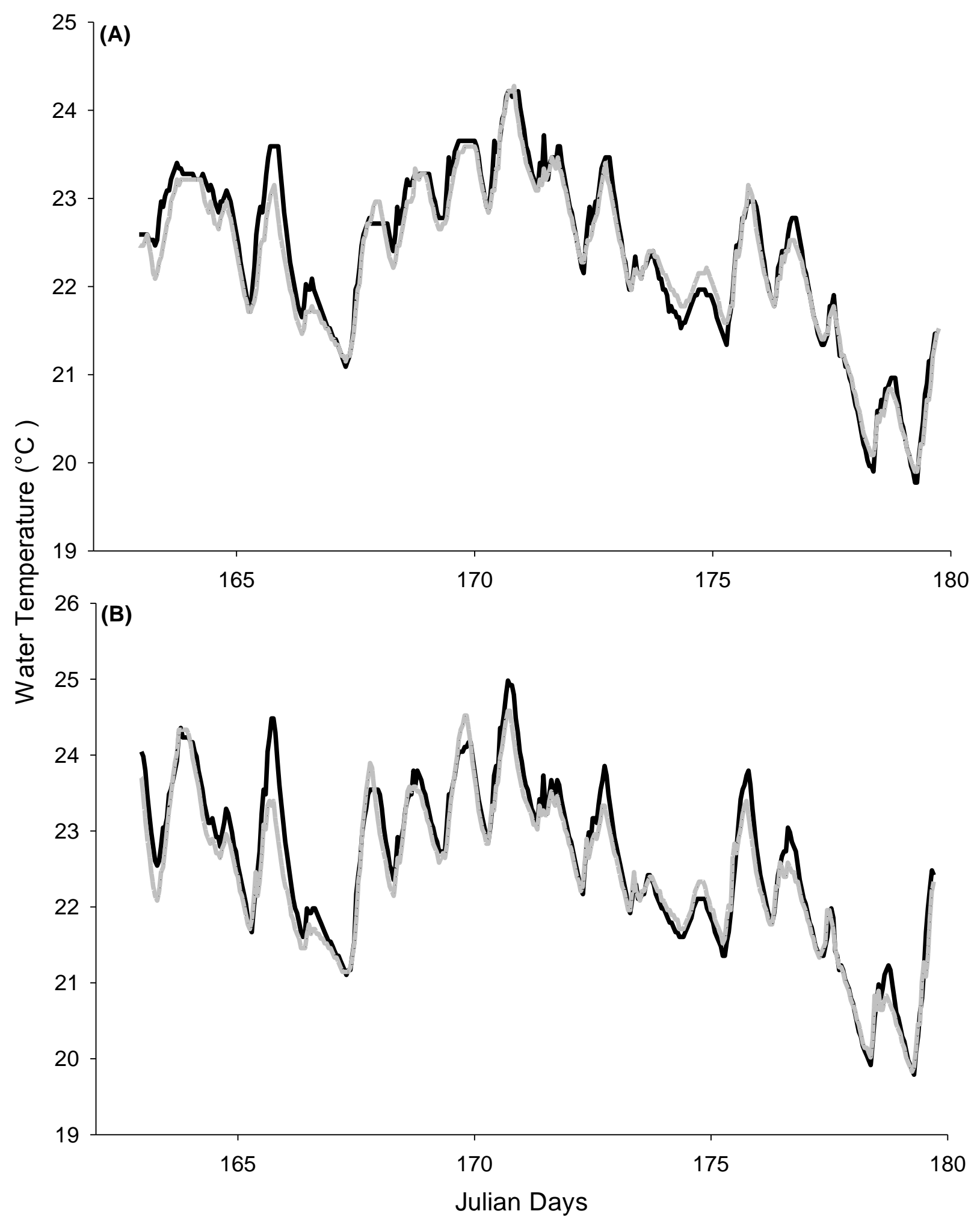

Figure 2.9: Temperature difference between control (black line) and experimental (grey line) vertical thermistor chains (shallow spot). Subpanels represent different depths; (A) $0.5 \mathrm{~m}$ from bottom, (B) $0.25 \mathrm{~m}$ from top. 


\subsubsection{Horizontal Thermistor Chain Temperature Measurements}

Horizontal thermistor chains were placed below the heating system and only pulled out after the end of the experiment (June $28^{\text {th }}$; Table 2.2). In the West location, heating was observed 0 to $4 \mathrm{~m}$ from the curtain (Fig. 2.10A-D), while similar temperatures were observed 4, 5, and $7 \mathrm{~m}$ from the curtain (Fig. 2.10E-F and H). At $7 \mathrm{~m}$, the experimental section had consistently lower temperature than the control section. In the East location, heating was observed at 0 to $4 \mathrm{~m}$ from the curtain (Fig. 2.11A-D), however, to a lesser extent than the West location. Temperatures were observed 4 and $5 \mathrm{~m}$ from the curtain were similar throughout the experiment (Fig. 2.11EF). At 6 and $7 \mathrm{~m}$ from the curtain, mid-afternoon temperature in the experimental section were higher, however, temperatures were similar thorough the rest of the day (Fig. 2.11G-H).

Table 2.2: Water temperature data $\left({ }^{\circ} \mathrm{C}\right)$ of vertical thermistor chains of all sections in all locations at all depths measured. Values are presented as mean $\pm \operatorname{SD}(n)$.

\begin{tabular}{ccc}
\hline Location/Depth & Control & Experimental \\
\hline East Transect & & \\
0 meters & $16.3 \pm 0.9(549)$ & $19.1 \pm 1.4(549)$ \\
1 meters & $11.1 \pm 0.3(549)$ & $19.4 \pm 1.4(549)$ \\
2 meters & $12.2 \pm 0.4(549)$ & $19.8 \pm 1.5(549)$ \\
3 meters & $19.2 \pm 1.3(549)$ & $20.3 \pm 1.5(549)$ \\
4 meters & $20.7 \pm 1.6(549)$ & $20.7 \pm 1.6(549)$ \\
5 meters & $21.1 \pm 1.6(549)$ & $21.1 \pm 1.6(549)$ \\
6 meters & $21.2 \pm 1.6(549)$ & $20.7 \pm 1.5(549)$ \\
7 meters & $21.3 \pm 1.6(549)$ & $21.4 \pm 1.7(549)$ \\
8 meters & $21.6 \pm 1.7(549)$ & $21.2 \pm 1.7(549)$ \\
9 meters & $21.8 \pm 1.8(549)$ & $21.4 \pm 1.7(549)$ \\
& & \\
West Transect & & $16.5 \pm 0.7(549)$ \\
0 meters & $15.8 \pm 0.8(549)$ & $17.6 \pm 0.9(549)$ \\
1 meters & $16.5 \pm 1.3(549)$ & $20.6 \pm 1.5(549)$ \\
2 meters & $20.2 \pm 1.4(549)$ & $21.1 \pm 1.6(549)$ \\
3 meters & $20.5 \pm 1.5(549)$ & $21.2 \pm 1.6(549)$ \\
4 meters & $20.9 \pm 1.6(549)$ & $21.3 \pm 1.7(549)$ \\
5 meters & $21.2 \pm 1.6(549)$ & $21.5 \pm 1.7(549)$ \\
6 meters & $21.4 \pm 1.7(549)$ & $21.8 \pm 2.0(549)$ \\
7 meters & $21.3 \pm 1.7(549)$ &
\end{tabular}



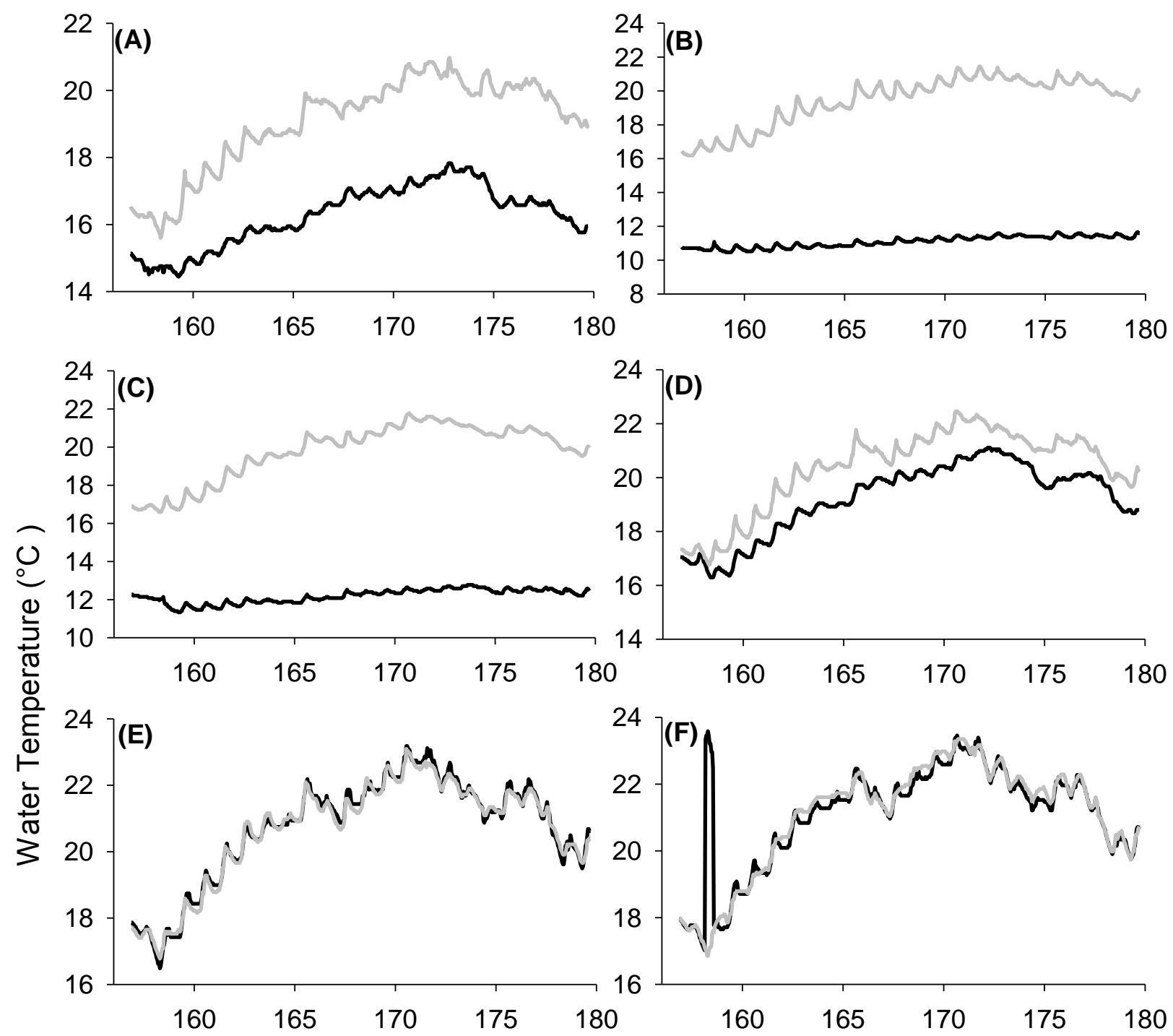

(D)

22
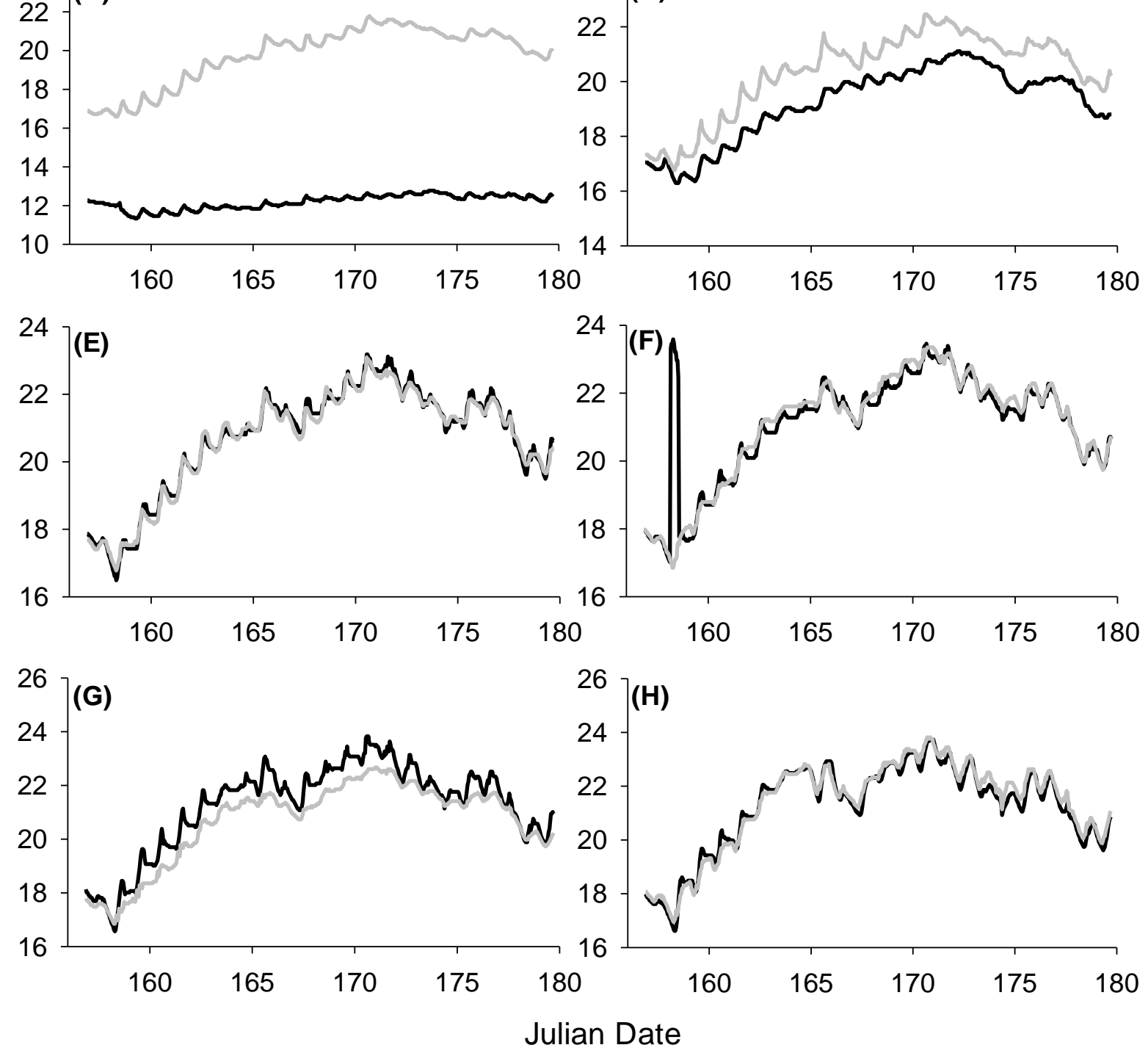

Figure 2.10: Temperature difference between control (black line) and experimental (grey line) horizontal thermistor chains (West location). Subpanels represent different distance from curtain; (A) 0, (B) 1, (C) 2, (D) 3, (E) 4, (F) 5, (G) 6, and (H) $7 \mathrm{~m}$. 


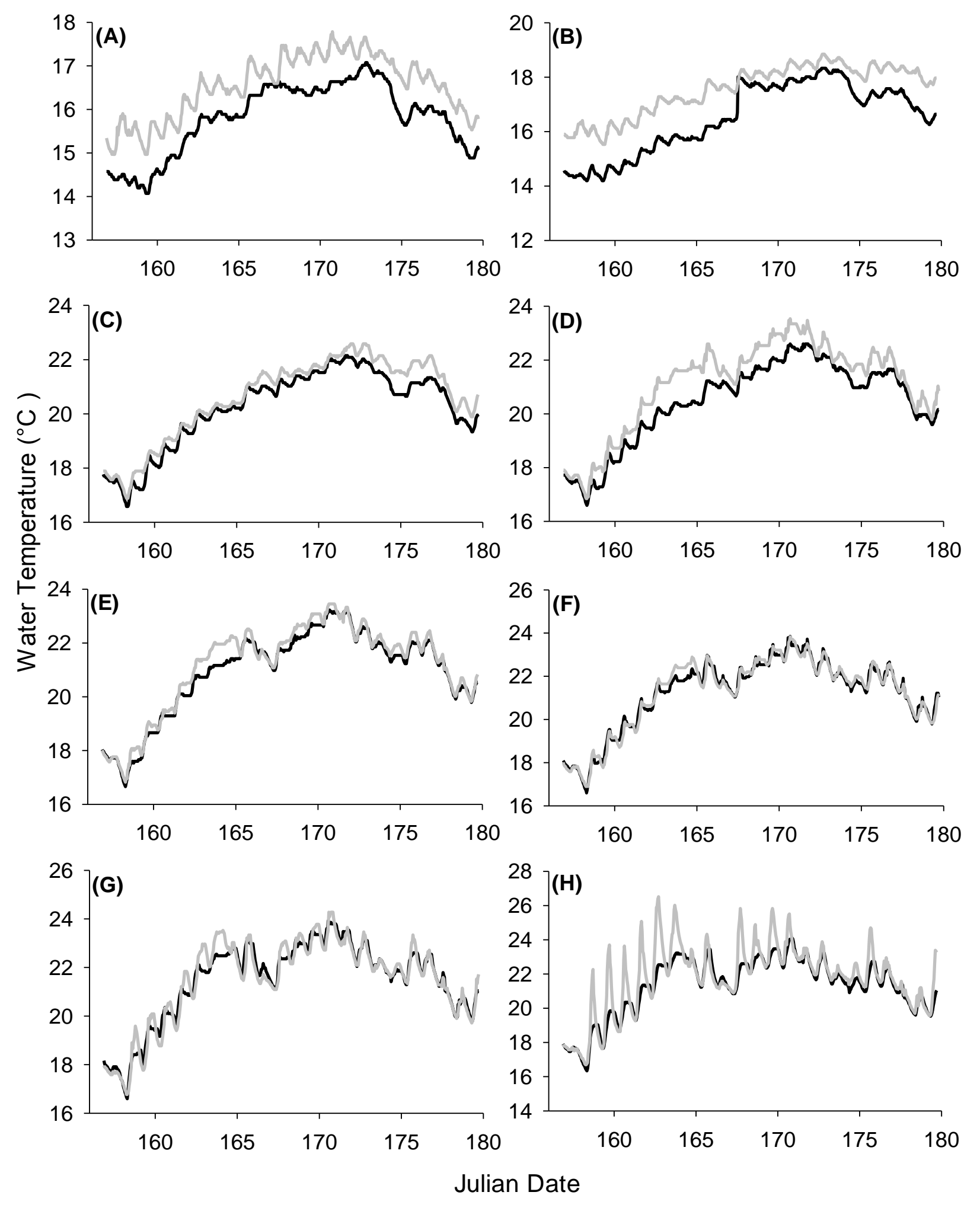

Figure 2.11: Temperature difference between control (black line) and experimental (grey line) horizontal thermistor chains (East location). Subpanels represent different distance from curtain; (A) 0, (B) 1, (C) 2, (D) 3, (E) 4, (F) 5, (G) 6, and (H) $7 \mathrm{~m}$. 


\subsubsection{Sample Collection}

All samples were collected, as scheduled, between June $5^{\text {th }}$ and $28^{\text {th }}$, however, due to the insufficient heating in the experimental treatment, samples were stored but not analyzed.

Nutrient concentration in the two mesocosms and the ambient section were determined but are not analyzed or discussed but are presented in Appendix 3. 


\section{$\underline{\text { 2.4 Discussion }}$}

Climate change, driven by anthropogenic emissions (Cook et al. 2016), has accelerated increases in GMST in recent decades (Hartmann et al. 2013). The projected GMST for the end of the century may be up to $4.8^{\circ} \mathrm{C}$ warmer, depending on emission levels (Collins et al. 2013). Water temperature around the globe, similarly, are expected to increase (Collins et al. 2013), which poses a significant threat to freshwater ecosystems (Woodward et al. 2010). Freshwater phytoplankton are key producers of essential PUFA, such as EPA and DHA (Hixson et al. 2015), that are necessary for maintain the health of aquatic and terrestrial organisms (Arts et al. 2009). Small-scaled laboratory studies have shown the decreased production of PUFA in response to higher temperatures (Renaud et al. 2002; Sushchik et al. 2003b; Fuschino et al. 2011; Pasquet et al. 2014), however, the response has not been observed in more complex natural system. Overall, large-scale temperature manipulation experiments in freshwater systems have not been conducted (Stewart et al. 2013), but are necessary to confirm responses to higher temperatures seen in labroatory and mesocosm experiments within the biological complexity of a natural system.

The objective of this experimental setup was to increase the water temperature of a large freshwater pond mesocosm ( 300,000 L) $4^{\circ} \mathrm{C}$ above the control mesocosm of similar volume in order to simulate predicted temperature on a freshwater ecosystem. Large mesocosms were created by dividing a small pond with several insulating pond curtain to create three large sections. A PVC/aluminum piping system attached to an electrical pool heater and pump was deployed $0.5 \mathrm{~m}$ from the pond bottom to increase the water temperature of the experimental section. Multiple temperature measurements demonstrated warming only occurred near the heating system (i.e., bottom of the pond) but not throughout the entire mesocosm. YSI measurements taken at 6 and $15 \mathrm{~d}$ into the experiment demonstrated a $\sim 4.5^{\circ} \mathrm{C}$ (Fig. 2.5) and $\sim 4^{\circ} \mathrm{C}$ (Fig. 2.6) increase in water temperature near the bottom of the pond, respectively. Vertical thermistor chains were examined to confirm warming, however overall, little to no differences were seen between control and experimental depths in corner (Fig. 2.7), middle (Fig. 2.8), and shallow (Fig. 2.9) spots. In the corner spot (i.e., deepest point in the section), the experimental section (at $0.5 \mathrm{~m}$ from the bottom) was on average $0.7^{\circ} \mathrm{C}$ warmer during the last seven days of the experiment (Fig. 2.7A), suggesting some warming achieved by the heating system. From 
Julian Date 158 to 164 , the control corner $2 \mathrm{~m}$ from the bottom was on average $0.5^{\circ} \mathrm{C}$ warmer than the experimental (Fig. 2.7D), however, temperatures near the surface were similar (Fig. 2.7E).

The vertical temperature profile of the middle of all three basins were examined (Fig. 2.8). Near the bottom, the middle of the control and experiment section were similar to each other but warmer than the ambient section (Fig. 2.8A-C). Near the surface, the middle area of the ambient and control sections was warmer than the experimental middle, especially from noon to early morning where temperature differences were typically $>1{ }^{\circ} \mathrm{C}$ (Fig. 2.8D). The position of the experimental section relative to the sun and the overhanging canopy cover may caused the experimental section to experience less warming from the sun. The middle section of the ambient and control section would receive more sunlight during near dawn and dusk, respectively. The shallow regions of both the control and experimental sections, which had similar levels of shading, showed little differences in temperature (Fig. 2.9). Horizontal thermistor chains (deployed underneath the heating system) demonstrated warming of the bottom of the pond, especially near the curtains (Figs. 2.10A-D and 2.11A-D).

Overall, the experimental setup described in this chapter was unable to sufficiently manipulate temperatures to mimic future climate warming scenarios; heating was observed, at depth, near the heating system but not throughout the entire water column. Previous studies, using smaller mesocosms, have used submerged heating elements (e.g., Liboriussen et al. 2005) or hot water pipes (i.e., McKee et al. 2000) at the bottom of the mesocosms. Water flow generated by pumps (McKee et al. 2000) or manual stirring with paddles (Liboriussen et al. 2005) was used to prevent a vertical gradient as locally high temperatures were observed near the mesocosm bottom. However, at a large scale, both of these methods to distribute heat become unfeasible and were not attempted.

Three major limitations of my experimental setup were the: a) piping system, b) mesocosm volume, and, c) power of pool heater. The indirect method of using a PVC/aluminum piping system to distribute heat into the pond was chosen to eliminate the effect of exposure to extremely high temperatures typical of pool heaters. Directly pumping in pond water into the pool heater, regardless of heat-generating ability, would result in more effective transfer of heat into the pond. Electrical pool heaters allow water to come in contact with a metal heating 
element and water temperatures within a pool heater can reach $40^{\circ} \mathrm{C}$. Therefore, pond organisms such as phytoplankton and zooplankton would experience substantially higher temperatures than intended. Although the PVC/aluminum piping system avoided such unnaturally high temperatures, heat transfer was insufficient to warm the large volume of water in the experimental mesocosm. Reducing the volume of the mesocosms may have allowed the same experimental setup to achieve warming, especially in shallower areas of the pond. Finally, the power of the heater was limited by the power supply available. Both the pool heater $(21 \mathrm{~A} ; 240 \mathrm{~V})$ and pool pump $(10 \mathrm{~A} ; 230 \mathrm{~V})$ used required significant power input. The pond was located near a private residence and was electrically equipped to handle the K-10 and K-15 model, which required higher amperage of power (42A and 63A, respectively). The combination of all three factors likely played a major role in heating near the bottom but not throughout the water column. However, the experimental setup attempted and, in particular, the sampling parameters and methodology utilized may benefit future studies on planning measurable responses to warming temperatures in a natural system.

Phytoplankton, biofilm, zooplankton, insect larvae were all collected with the goal of assessing the effect of warmer temperatures on PUFA production and transfer through an aquatic ecosystem. Community structure, at all trophic levels, is an important factor to consider both independently and in combination with FA analysis in the context of climate warming. Temperature-mediated increases in individual growth rates have the potential to lead to a temporal mismatch and uncoupling between trophic levels (Winder \& Schindler 2004), which can be detected by examining community structures of several trophic levels. Elevated temperatures also shift entire communities of phytoplankton and zooplankton communities towards smaller and fast-growing species (Rasconi et al. 2015, 2017). For example the predicted dominance of PUFA-poor cyanobacteria at the base of an aquatic food web as the climate heats up (Paerl and Huisman 2008) has the potential to reduce the bioavailability of EFA in all aquatic trophic levels. This effect may also cross the aquatic-terrestrial boundary as emerging insects represent an important EFA source for terrestrial predators.

Sorting of most trophic level by taxa, prior to FA analysis, is an important step to determine the taxa-specific effects of higher temperatures as differences between FA profiles have been demonstrated between orders of zooplankton (e.g., Persson \& Vrede 2006; Smyntek et 
al. 2008; Burns et al. 2011), insect larvae species (e.g., Makhutova et al. 2017), and orders of adult insect (e.g., Martin-Creuzburg et al. 2017).

Most samples collected for FA were intended to be lyophilized prior to analysis, however, recent advances in NMR spectroscopy (Bastawrous et al. 2018) has allowed the analysis of contaminant-induced sub-lethal effects on D. magna metabolites and the potential biochemical processes that may be affected (Kovacevic et al. 2018; Wagner et al. 2018). Although samples were not analyzed, the cage methodology of incubating lab-grown D. magna allowed most individual to survive and therefore, in-vivo analysis of Daphnia metabolomics could have provided a novel technique of measuring the effects of climate warming. Water sampling was essential in examining the effect of higher water temperatures on nutrients and contaminates that biological samples were exposed to in the water column. Higher temperatures increase phosphorus release directly from sediment, as well as indirectly by increasing biological activity at the sediment-water interface (Jiang et al. 2008). Higher nutrient availability in the water column may also support the domination of cyanobacteria in freshwater ecosystems. Similar to nutrients, methyl mercury production and release into the water column also increase with warmer temperatures (Wright \& Hamilton 1982). Increasing temperatures have also been shown to increase the rate of mercury accumulation in rainbow trout (Salmo gairdneri; MacLeod \& Pessah 1973). This is particularly concerning for humans as rainbow trout are the major fish species produced in lake-based net pens in Ontario (Moccia \& Bevan 2018). Lake-based aquaculture has demonstrated to benefit feral fish populations by increasing productivity (Mills et al. 2008), however, warmer temperatures in the future may also increase mercury concentrations in a commonly-eaten fish, which has known negative effects in humans (Mozaffarian 2009). Although the main objective of this study was to examine the ecological impacts of warming temperature, potential consequences to humans are plausible.

The effects of climate change on freshwater ecosystems have been studied in laboratories and small-scale mesocosm experiments, however, large-scale experiments encompass biological complexity that cannot be replicated at smaller scales. The difficulty of manipulating temperature at a large scale is evident due to the lack of studies in the literature. The usage of a PVC/aluminum piping system $0.5 \mathrm{~m}$ above the sediment did not successfully warm the experimental section $4^{\circ} \mathrm{C}$ above the control section, however, future studies may successfully manipulate temperature in a natural system using a higher capacity heater and/or smaller pond 
area/volume. In a non-residential area, propane tanks provide good alternative for a high-energy heater. For example, Budy (Utah State University, pers. comm.) and colleagues have initiated a climate change experiment in 4 lakes (two controls and two experimental) in Alaska to examine the effects of warming on freshwater organisms. In the experimental ponds, a swimming pool heater attached to several large propane tanks (650-pound) takes in lake water, warms it up to $\sim 30^{\circ} \mathrm{C}$ and circulating it back into the lake to increase the experimental ponds $\sim 4^{\circ} \mathrm{C}$ above control water lake water temperatures. A commercial SolarBee ${ }^{\circledR}$ solar-powered mixer also mixes the lake water, distributing the heat thorough the water column. The experimental setup described was ultimately successful in warming up the experimental lakes, however, physiological effects associated with microorganisms entering the heaters remain unknown.

Regardless of the methods, temperature manipulation experimental designs all have benefits and drawbacks, however, the experimental results presented in this chapters may potentially be used in future experimental designs that attempt to warm waterbodies indirectly in order to conduct an in-situ temperature manipulation experiment. 


\section{Chapter 3}

Seasonality and Depth Influences the Quantity and the Nutritional Quality of Aquatic Biofilm

\section{$\underline{3.1 \text { Introduction }}$}

In freshwater ecosystems, aquatic biofilm can represent a large portion of the primary productivity; in some cases, more than pelagic phytoplankton (Vadeboncoeur \& Steinman 2002). Similar to pelagic phytoplankton, aquatic biofilms are involved in several ecosystem processes, such as elemental nutrient cycling (Paul et al. 1991; Lyon and Ziegler 2009) and providing energy input at the base of food webs (Simon et al. 2003). Stable isotope tracer studies have demonstrated the importance of biofilms to invertebrates (Lau et al. 2009) and fish (Vander Zanden \& Vadeboncoeur 2002). The nutritional quality of biofilm to its consumers has also been assessed using stoichiometric ratios of carbon, nitrogen, and phosphorus (e.g., Hillebrand et al. 2004; O’Brien \& Wehr 2009). And, FA, due to their importance to grazers, have been used as another indicator of nutritional quality (Saikia 2011; Guo et al. 2016b). Therefore, abiotic factors (e.g., temperature, light, and nutrients) that can potentially influence the biofilm quantity and more recently, FA composition continues to be the focus of investigation (e.g., Guo et al. 2016b).

Abiotic factors, such as temperature, underwater light spectra, and nutrients have been shown through manipulative studies, independently and interactively, to influence biofilm quantity and FA content (see Chapter 1). Temperature generally increases biofilm biomass in streams (e.g., Descy \& Mouvet 1984) and lakes (e.g., Mahdy et al. 2015), up to a certain thermal limit (Patrick 1971). The effect of temperature on the FA composition of biofilm has not been directly studied, although stream-collected biofilm from summer (i.e., warmer temperatures) typically lead to a higher proportion of SFA and lower proportions of PUFA (e.g., Guo et al. 2015). Similar to studies on pelagic phytoplankton, colder temperatures (observed in fall and winter) causing cells to undergo homeoviscous adaptation, which shifts membranes towards being PUFA-dominated in an effort to maintain fluidity (Sushchik et al. 2004). Elevated light levels typically increases biofilm biomass (e.g., Wellnitz et al. 1996), especially algal cells in the community, however, it has also been shown to decrease PUFA content of the community (Cashman et al. 2013). Nutrients (i.e., phosphorus and nitrogen) are essential for cell growth and increase biofilm biomass in streams (e.g., Stockner \& Shortreed 1978) and lakes (e.g., Marks \& Lowe 1993), as well as increase the proportion of PUFA (Hill et al. 2011; Guo et al. 2016c). The 
abiotic factors discussed above vary seasonally (Rosemond 1994), which can strongly influence aquatic biofilm growing in natural systems.

Seasonal variability in the quantity of aquatic biofilm has been demonstrated using both artificial and natural substrates and with reference to the changing abiotic factors that are observed between seasons (Rosemond 1994; McCormick et al. 1997; Godwin \& Carrick 2008). The optimal combination of nutrients, light, and temperature in freshwater systems typically produces maximum biofilm biomass in spring and late summer/early fall while minimal biomass is observed in winter months (Castenholz 1960; Round 1961; Klarer \& Hickman 1975; Hickman 1978a; Eloranta 1982). Dimitic lakes undergo turnover in spring and fall which typically increases the availability of elemental nutrients, such as phosphorus, required for microbial growth within a biofilm (Ferragut et al. 2010). Higher PAR in the summer months can also increase algal growth within a biofilm community as light typically increases photosynthetic activity (Hill 1996), which has been mostly observed as an higher chl $a$ content of stream biofilm from open sites compared to shaded sites (DeNicola et al. 1992; Steinman 1992; Larned \& Santos 2000). Increased algal growth can also indirectly increase bacterial biomass (Murray et al. 1986; Espeland et al. 2001) due to their symbiotic relationship with algal cells. Warmer temperature in the summer months increases the rates of microbial metabolism (Toseland et al. 2013) and growth (Butterwick et al. 2005). However, high light and temperatures (characteristic of summer conditions) also typically leads to the dominance of thermally-tolerant cyanobacteria in biofilm communities (van der Grinten et al. 2005).

Seasonal changes in algal community composition and cellular temperature adaptation play a large role in explaining seasonal variation in biofilm FA composition. In this context, seasonal variation has been demonstrated in the FA composition of freshwater stream biofilm (Sushchik et al. 2010; Honeyfield \& Maloney 2015; Richoux et al. 2018). Sushchik et al. (2010) related the change in biofilm FA composition to the seasonal change in the algal community within the biofilm. Pelagic cyanobacteria that dominant in summer months typically have lower PUFA content (Guedes et al. 2011; Strandberg et al. 2015) than pelagic diatoms, which typically thrive in spring and fall and have higher PUFA content, especially EPA (Ahlgren et al. 1992; Lang et al. 2011). The seasonal change in biofilm PUFA content has also been hypothesized to be due to temperature adaptations of the algal populations (Sushchik et al. 2010; Honeyfield \& Maloney 2015). Multivariate analysis revealed that temperature was a main driver of summer 
and fall biofilm FA profiles (Honeyfield \& Maloney 2015). Warmer summer temperatures may induce PUFA-dominated membranes to shift to SFA-dominated membranes, helping to explain the lower PUFA content observed in summer biofilm (Sushchik et al. 2010). Thus, it is evident that light, nutrients, and temperature can vary seasonally and influence aquatic biofilm, however, the same abiotic factors can also vary within-season along a depth gradient and also affect aquatic biofilm.

Light, temperature, and nutrients can greatly vary along a depth gradient, and which has been documented in shallow freshwater lake systems, especially during the summer months (Wetzel 2001). The underwater light spectra (i.e., quantity and quality of light) is modified through the water column as light is scattered/absorbed by, a) water, b) dissolved organic matter, c) suspended inorganic particles, and, d) pelagic phytoplankton (Gallegos \& Moore 2000). Thus, the increase in pelagic phytoplankton growth and biomass during summer months typically supresses the growth of biofilm (Hansson 1988, 1992; Sand-Jensen \& Borum 1991; Havens et al. 2001a; Vadeboncoeur et al. 2001) and this suggests that pelagic phytoplankton play key role in determining light availability for underlying biofilm communities. Studies examining depthrelated effects of biofilm typically observe greater algal biomass (measured via chl $a$ content) when biofilm is growing near the water surface, especially during spring/summer months (Liboriussen \& Jeppesen 2009; Kreuzinger-Janik et al. 2015) and relate it to greater PAR irradiance at shallower depths (Yang and Flower 2012; Schroeder et al. 2013).

Water temperature also typically decreases with depth in wetland and lakes as thermal stratification occurs during summer months as warm surface waters are separated from cooler deep waters, with transitional temperatures in-between (Ullyott \& Holmes 1936). Although considered a common occurrence in lakes, shallow ponds $(<3 \mathrm{~m})$ can undergo stratification in summer months with temperatures differences up to $3-4^{\circ} \mathrm{C}$ between warm surface and cool bottom layers (Song et al. 2013). Summer stratification can lead to anoxic conditions at the sediment-water interface, which can stimulate the release of inorganic nutrients into the water column (Golterman 1995) and therefore, biofilm closer to the sediment may be exposed to higher nutrient concentrations. Dimitic lakes (i.e., those that mix twice a year) have relatively uniform nutrient concentrations within the water column during spring and fall turnover as layers are mixed (Wetzel 2001). Although differences in abiotic factors between depths within a season 
may not be as drastic as between seasons, there is evidence that depth does dictate biofilm biomass in freshwater systems (discussed below).

The effect of depth on biofilm quantity has been studied, but the response varies depending on the substrate type. The epiphyton (i.e., biofilm growing on plants) biomass response to depth, in low-nutrient lakes, is generally positive while a negative relationship typically occurs in high-nutrient lakes (Evans \& Stockner 1972; Khondker \& Dokulil 1988; Lalonde \& Downing 1991; Yang \& Duthie 1995; Kahlert et al. 2002). Epipelic (biofilm growing on muddy sediments) and epipsammic (growing on sand) biofilm typically demonstrate a negative relationship with depth (Round 1961; Stevenson \& Stoermer 1981; Stevenson et al. 1985; Sundbäck \& Jönsson 1988; Yang \& Duthie 1995; Nozaki et al. 2002; Yang \& Flower 2012). Epixylic (growing on wood; Rouf et al. 2010) and epilithic (growing on rocks; Quinn \& Hickey 1994) biofilm biomass also typically demonstrate a negative relationship with depth. These substrate-dependent effects of depth on biofilm biomass can be removed through the uniform application of artificial surfaces, such as unglazed tiles or strips of inert plastic.

Artificial surfaces typically misrepresent natural communities as they can underestimate both biomass (Cattaneo \& Amireault 1992) and species richness (Barbiero 2000) compared to natural surfaces. However, due to the high replicability of artificial surfaces (Barbiero 2000) they have been used to clearly demonstrate that lower biofilm biomass is typically observed at greater depths (Liboriussen \& Jeppesen 2006, 2009; Sánchez et al. 2013; Kreuzinger-Janik et al. 2015). This decrease in biofilm biomass at greater depths is typically linked with the decreasing algal biomass (Liboriussen \& Jeppesen 2009; Kreuzinger-Janik et al. 2015) due to diminishing light availability vertically in the water column (Yang \& Flower 2012; Schroeder et al. 2013). The effect of depth on biofilm biomass has typically been studied in the context of grazers, however, limited work has examined the nutritional value (standardized for organic biomass) of depthdistributed biofilm, especially in terms of the physiologically-important PUFA.

Epilithic biofilm collected from deeper sites have been shown to contain higher nitrogen and phosphorus content, suggesting higher elemental (stoichiometric) nutritional quality (Kahlert et al. 2002). However, the effect of depth on PUFA content (as an indicator for nutritional quality), has not been fully explored despite the significance of PUFA to grazers. Common grazers of biofilm include snails, caddisflies, chironomids, mayflies, protists, and crustaceans 
(Mazumder et al. 1989; Bronmark et al. 1992; Blumenshine et al. 1997; Böhme et al. 2009). High snail abundance has been observed on tiles incubated at greater depths (Schroeder et al. 2013), which may be due to the preference of snail abundance and activity on surfaces sheltered from high velocities and irradiance (DeNicola \& McIntire 1991). Nematode communities may also be depth-regulated as algae-feeding nematode species dominate shallow waters $(<60 \mathrm{~cm})$ whereas bacteria-feeding species dominate deeper waters $(80-300 \mathrm{~cm}$; Peters et al. 2012). The abundance of insect larvae, such as chironomids (Thorp \& Diggins 1982), caddisflies (Thorp \& Diggins 1982), and dragonflies (Wissinger 1988) may be related to depth as higher abundance are typically observed at shallower depths. This depth distribution of grazers may be related to the higher biofilm biomass typically observed at shallower depths (discussed above) and/or the nutritional value, measured by the PUFA content.

The importance of PUFA to biofilm grazers can be demonstrated by the accumulation of PUFA in the tissue of biofilm grazers such as snails (Wacker 2005; Ekin et al. 2011), crustaceans (Bell et al. 1994; Sushchik et al. 2003a), and the larvae of chironomids (Hanson et al. 1985; Bell et al. 1994; Ghioni et al. 1996; Sushchik et al. 2003a; Makhutova et al. 2011), mayflies (Bell et al. 1994; Sushchik et al. 2003a), stoneflies (Bell et al. 1994; Ghioni et al. 1996), and caddisflies (Sushchik et al. 2003a). The ability of insect larvae to biosynthesize LC-PUFA (e.g., EPA and DHA) from C18 PUFA precursors appears to be taxa-specific (Hanson et al. 1983; Dadd et al. 1987; Goedkoop et al. 2007; Torres-Ruiz et al. 2010). However, controlled lab experiments demonstrate that only diets supplemented with PUFA are sufficient for optimal insect larvae growth and reproduction (Hanson et al. 1983; Cargill et al. 1985). The importance of physiologically-important FA in insect larvae can not only determine the PUFA availability for higher trophic levels, but also affect terrestrial predators in riparian habitats surrounding freshwater systems. Amphibiotic insect larvae represent a key vector for the export of PUFA from aquatic to surrounding terrestrial systems. Recently, chironomids and dragonflies have been shown to represent a large subsidy of PUFA to the terrestrial areas surrounding lakes (MartinCreuzburg et al. 2017; Popova et al. 2017), and, as such, have demonstrated the importance of emerging aquatic insects to terrestrial predators (Twining et al. 2016; Fritz et al. 2017). Odonates (i.e., dragonflies and damselflies) emerging from forested areas near a large freshwater lake were equal to the biomass estimates of herbivorous terrestrial insects, $0.30 \mathrm{~g} \mathrm{C} \cdot \mathrm{m}^{-2} \cdot$ year $^{-1}$ versus $0.11-0.70 \mathrm{~g} \mathrm{C} \cdot \mathrm{m}^{-2} \cdot$ year $^{-1}$, respectively. However, unlike terrestrial insects, which are known 
to contain low PUFA content (Hixson et al. 2015), odonates were responsible for an average export of $1.92-11.76 \mathrm{mg}$ EPA+DHA $\cdot \mathrm{m}^{-2} \cdot \mathrm{y}^{-1}$ over a 31-year period (Popova et al. 2017).

The overall biomass and PUFA export of insects emerging from waters has been also shown to decrease with water depth. A steady decline in the export of total and multiple individual PUFA (e.g., LIN, ARA, and EPA) was observed from 0 to $6 \mathrm{~m}$, after which the export stabilized (Martin-Creuzburg et al. 2017). The decline in the export of PUFA from emerging insects from deeper waters may be related to potential nutritional differences in aquatic biofilm that they graze upon. Therefore, the depth-related differences in the quantity and, more notably, the nutritional quality of aquatic biofilms may potentially be important in determining the direct and indirect availability of nutrients for aquatic and terrestrial ecosystems, respectively.

The main objective of this study was to examine the effect of season and depth on the quantity and nutritional quality (i.e., PUFA) of aquatic biofilm. Spatial variability has been demonstrated in biofilm biomass, stoichiometry, and FA profiles (O'Brien and Wehr 2009; Richoux et al. 2018) and therefore, this study also considered the possible spatial variability associated with collected biofilm. Unglazed ceramic tiles were incubated on 18 incubation platforms suspended at six different depths $(25,50,75,100,125$, and $150 \mathrm{~cm}$ from the surface). Incubator devices were deployed along three transects (to account for spatial variability) in a randomized block design during summer and fall months (to account for seasonal variability).

Dry weight (DW) and ash-free dry weight (AFDW) were used as an ex-situ measure of biofilm biomass. Destructive sampling (i.e., DW and AFDW) are used regularly to determine biofilm biomass, however, confocal laser scanning microscopy (CLSM) can potentially reveal differences in in-situ structural properties that are lost in destructive sampling (Albers 2010). The usage of CLSM has increased in environmental research (Lawrence et al. 2007) and has extended to assess the effect of abiotic factors, such as nutrients, light, temperature and contaminates, on the three-dimensional structural profile of aquatic biofilm (Lawrence et al. 2004; Neu et al. 2005; Proia et al. 2012; Zhao et al. 2018). Similar to previous literature (e.g., Proia et al. 2012), bio-volume and mean thickness will be used as indicators of in-situ biofilm biomass (combined referred to as structural profile). Bio-volume represents the overall volume of the biofilm while mean thickness represents the spatial size (i.e., height) of the biofilm and is used regularly in biofilm literature (Heydorn et al. 2000). Roughness coefficient has also been 
measured with environmental samples (Mueller et al. 2006) and is a measure of the variance of thickness measurements (Murga et al. 1995). This represents the structural heterogeneity of the biofilm and may be used as a measure of the effect of grazing on biofilm (Derlon et al. 2012, 2013). Grazing pressure creates greater peaks and valleys within a biofilm (i.e., grazing trails) and thus, a higher roughness coefficient represents increased surface heterogeneity, an indication of grazing which would not be detected when only destructive sampling methods are used.

Previous research has demonstrated a relationship between water depth and biomass, as well as stichometry ratios, however, PUFA were used as the main nutritional indicator in my study. Specifically, $\sum$ n-3 PUFA, $\sum$ n-6 PUFA, and EPA+DHA content were used as indicators of nutritional quality due to their importance to biofilm grazers (e.g., Torres-Ruiz et al. 2010). This study will not designate n-3 and n-6 PUFA as "essential" but "physiologically important" to grazers due to the wide range of taxa that graze on biofilm, each with specific FA physiological needs and biosynthesis/bioconversion abilities (Cunnane 2003). Based on the results of previous studies, I predicted that biofilm biomass (quantity) and PUFA content (nutritional quality) would decrease with increasing depth due, in part, to the high algal biomass that is typically observed near the water surface. I also examined the content and proportion of specific SFA, MUFA, and PUFA, which may reveal potential changes in the overall FA profile of aquatic biofilm. The unsaturation index (UI) of biofilm is calculated by multiplying the content ( $\mu \mathrm{g} \mathrm{FA/mg} \mathrm{AFDW)}$ of a FA multiplied by the number of double bonds, and then summing for all FA identified in a particular sample, and has been used as an indicator of temperature adaptation in phytoplankton (Flaim et al. 2012, 2014; Anesi et al. 2016). The content of a single MUFA, oleic acid (18:1n-9), was also specifically examined due to its incorporation into membranes for biochemical adaptation to cooler temperatures (Bly et al. 1990; Teoh et al. 2004, 2013). Results from this study can provide reference data to document the lipid profiles of freshwater biofilm incubated along a depth gradient in two seasons, as well as attempt to explain depth-dependent differences in grazer abundance. 


\section{$\underline{\text { 3.2 Methods }}$}

\subsubsection{Study Site}

This study was conducted in a small mesotrophic freshwater pond in Whitby, Ontario, Canada (43 59' 58.1892" N, 79²' 9.9384" W, $260 \mathrm{~m}$ above sea level). The pond, although, man-made, has lain undisturbed for $>10 \mathrm{y}$, allowing a natural community to become established. The pond is $\sim 100 \mathrm{~m}$ long and varies in its width $\left(\sim 2,100 \mathrm{~m}^{2}\right)$ with an average and maximum depth of $2.92 \mathrm{~m}$ and $5.10 \mathrm{~m}$, respectively. The immediate forest surrounding the pond is composed of mixed birch and coniferous trees. The catchment of the pond was not determined, however, active farming is not found within a $1 \mathrm{~km}$ radius or immediately “downhill”. The pond is groundwater-fed as no surface water connections are visible, however, underwater connections are unknown. No specific permits were required for the described experiment. Experiment was performed on privately-owned land with prior consent from the landowners (Peter and Elaine Leigh).

Incubation platforms were deployed in a randomized block design; all six depths were randomly ordered along three separate transects $\sim 12 \mathrm{~m}$ apart to account and test for any spatial variability across the pond (Fig. 3.1). Incubators were secured at $1 \mathrm{~m}$ apart from each other to minimize shading between incubators. Nylon rope was used to tie incubators together and then the entire transect of incubators was secured to a tree on both sides of the pond with enough slack to only allow incubators to move $<1 \mathrm{~m}$ in any direction. Incubation devices were deployed for 68-70 d; summer biofilm was incubated from 11 July 2017 until 16-18 September 2017 while fall biofilm was incubated from 3 October 2017 until 9-11 December 2017. 


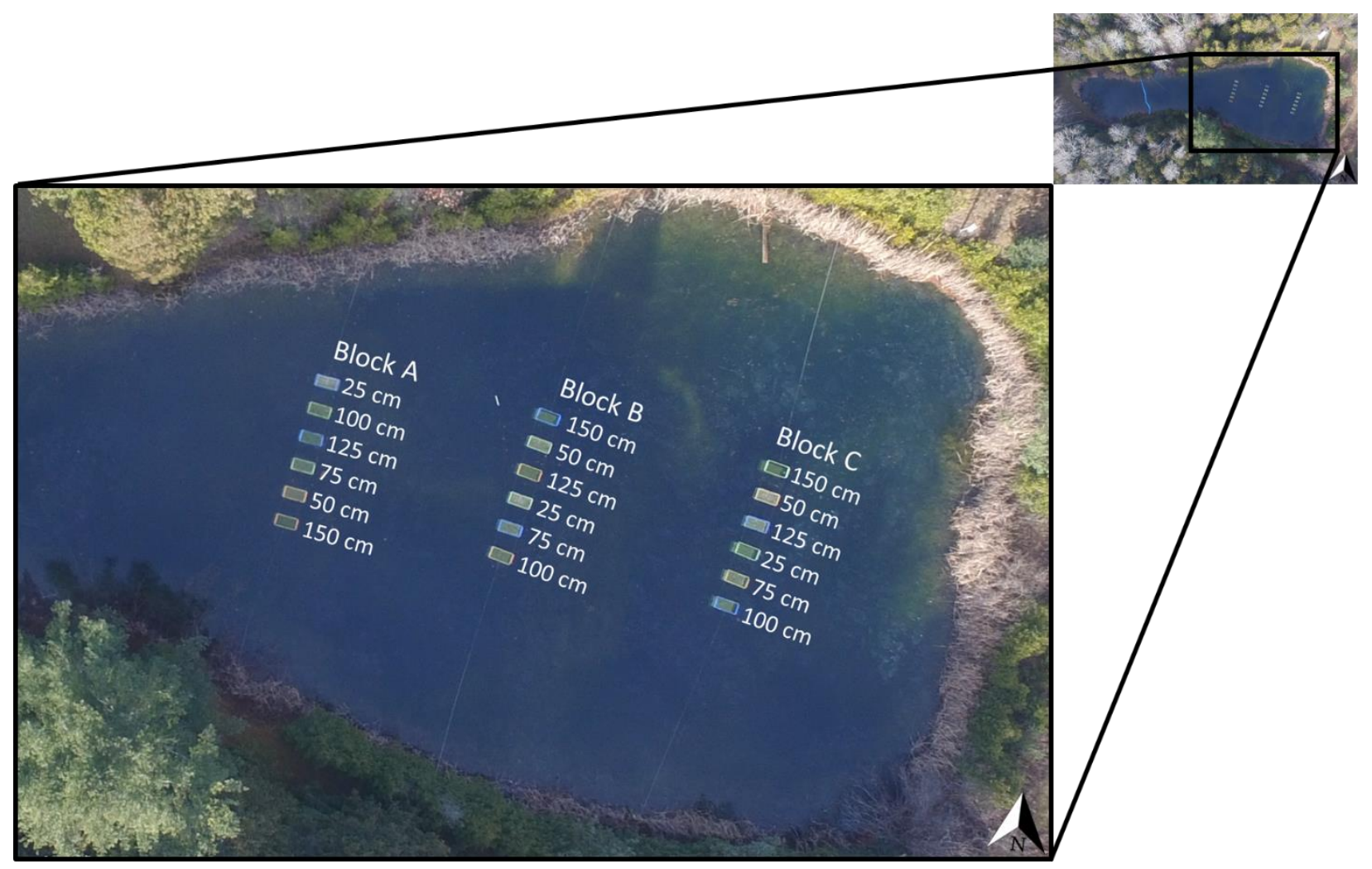

Figure 3.1: Aerial photograph of pond. Incubator platforms are deployed in a randomized block design along three transects (from left to right: Block A, B, and C). Photo taken with DJI Phantom 4 drone on December $4^{\text {th }}, 2017$ ( 1 PM). Photo credits: George Torrance.

\subsubsection{Biofilm Incubator Device Design}

The incubator device design was adapted from Huggins et al. (2004) with several modifications to adapt to the still water of a small pond rather flowing current of a river system. A square PVC frame was attached to a thin sheet of white HDPE, measuring $114 \mathrm{~cm} \times 60 \mathrm{~cm}$. A thin strip of HDPE was added around the boundary to prevent tiles from sliding off the device. Tiny HDPE squares were taped to the sheet to add a small space between each tile. Carabiners and metal chain were used to attach the HDPE sheet to a second, slightly larger, PVC frame held at the surface with pool noodles. The length of the metal chain determined the depth at which the tiles were incubated (i.e., 25, 50, 75, 100, 125, and $150 \mathrm{~cm}$ from the surface). Tiles in biofilm studies are typically incubated near the surface (i.e., $25 \mathrm{~cm}$; see Huggins et al. 2004), therefore, I used increments of $25 \mathrm{~cm}$ up to $150 \mathrm{~cm}$ (the lowest depth position ensured that all incubators were at least $1 \mathrm{~m}$ from the sediment). Each incubator device held 18 unglazed beige-colored ceramic tiles (Modern Tiles Plus, Richmond Hill, Canada) measuring $15.2 \mathrm{~cm} \times 15.2 \mathrm{~cm}(232.3$ $\mathrm{cm}^{2}$; Fig. 3.2). All tiles were pressure-washed (with tap water) prior to deployment in both 
seasons. Two submersible temperature loggers (Alpha Mach®; iBWetLand; AM015) were placed in Falcon tubes with several holes to allow temperature equilibrium; one iButton was attached to each short side of each incubator for hourly temperature measurements $\left(0.06^{\circ} \mathrm{C}\right.$ resolution).

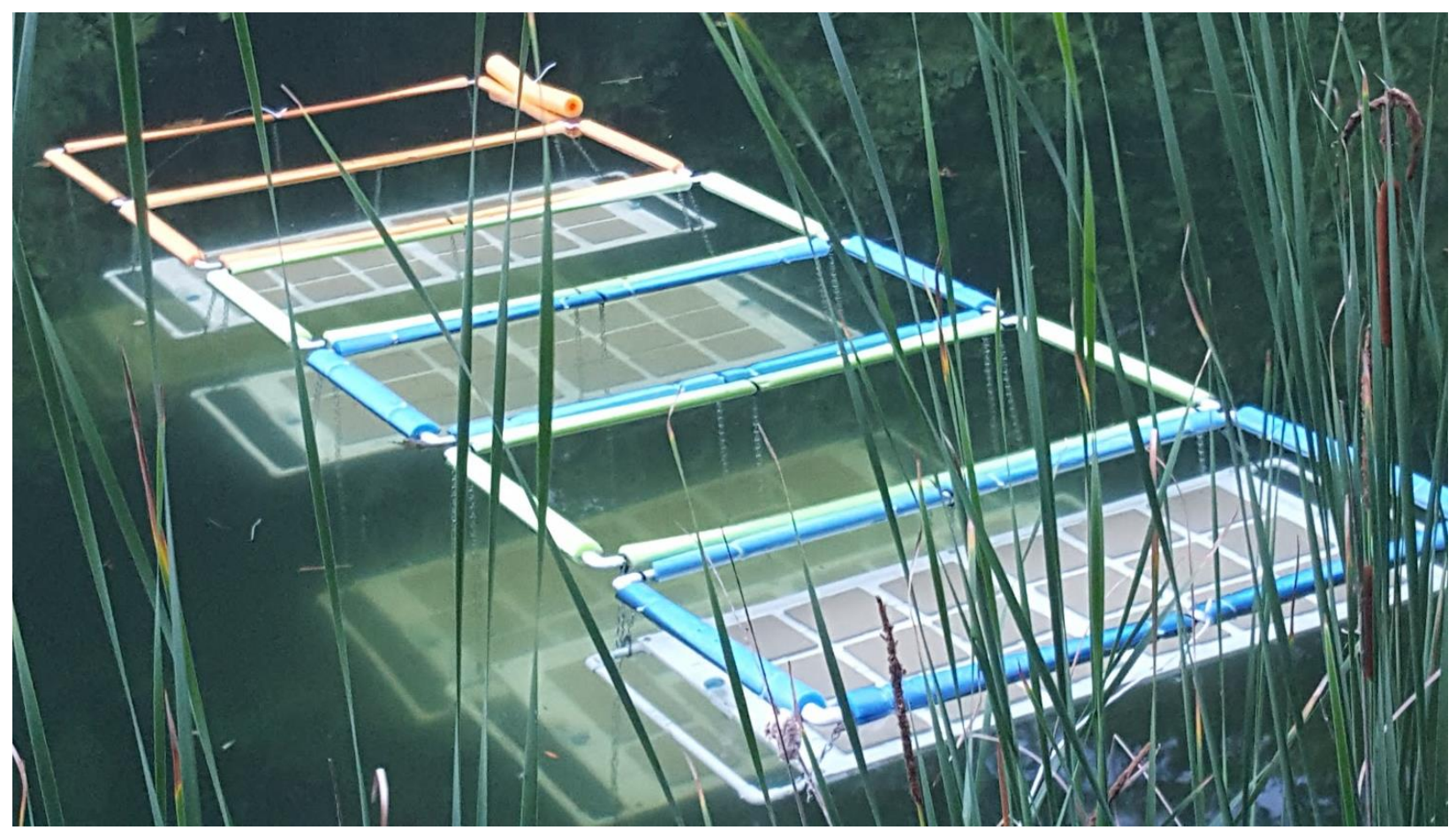

Figure 3.2: Unglazed ceramic tiles incubated on biofilm incubator devices deployed. Incubators were joined together for the picture but were moved into final position 1 meter apart.

\subsubsection{Abiotic Measurements}

Abiotic factors were measured weekly using a YSI@ EXO2 Multiparameter Sonde (Xylem@, 599502-00). The sonde was equipped with several probes and was able to measure water temperature $\left({ }^{\circ} \mathrm{C}\right)$, specific conductivity compensated to $25^{\circ} \mathrm{C}(\mu \mathrm{S} / \mathrm{cm})$, total dissolved solids (TDS; mg/L), pH, optical dissolved oxygen (ODO; \% saturated), and colored dissolved organic matter (CDOM; RFU). The sonde was lowered to the downwind side of the incubator (typically the west side) so that the probes were at the depth of the tiles. Triplicates were taken for each incubator and all measurements were taken between 8 AM and 12 PM.

Underwater light measurements were taken using an UV-VIS spectrometer (Ocean Optics®, Flame Spectrometer, FLAME-S-UV-VIS), a custom 3 m UV-VIS fiber cable (Ocean Optics®, Premium-grade Patch Cord, OCF-104852), and a cosine corrector (Ocean Optics®, 
cosine corrector, $\mathrm{CC}-3$ ) for $180^{\circ}$ measurements. The setup (i.e., spectrometer attached to a fiber optic cable equipped with a cosine corrector) was calibrated to measure absolute irradiance $\left(\mu \mathrm{W} / \mathrm{cm}^{2} / \mathrm{nm}\right)$ prior to each day of measurement using a deuterium-halogen calibration light source (Ocean Optics®, DH-3-CAL). The cosine corrector was filled with filtered pond water (vacuum-filtered using $0.2 \mu \mathrm{m}$ polyethersulfone membranes filters) to calibrate the spectrometer for underwater light measurements. The fiber optic cable was attached to a L-shaped ABS pipe ( $2 \mathrm{~m}$ by $1 \mathrm{~m}$ ) so that the cosine corrector was facing upwards (to measure downwelling irradiance) and was deployed $1 \mathrm{~m}$ from the boat to prevent shading. OceanView ${ }^{\mathrm{TM}}$ software (Ocean Optics®, Version 1.6.3) was used to record and process absolute irradiance measurements. Due to the spatial and temporal variability of sunlight, all measurements were taken from one central location in the pond (between Block B and C) to test the effects of depth and season on underwater light climate. During measurements the cosine corrector was always positioned closer to the sun with the boat in its shadow to prevent the boat from blocking the sun. A run consisted of three light measurements at each all six depths from 25 to $150 \mathrm{~cm}$; at least three runs were completed per day. All light measurements were taken on clear and sunny days within an hour of solar noon (i.e., when the sun was at the highest point on the horizon). During summer period, the spectrometer was unavailable, therefore, light measurements in the summer were only taken during the final three weeks prior to harvesting. During the fall period, weekly measurements were taken, however, the limited number of sunny days prevented regular light measurements.

\subsubsection{Biofilm Sampling/Harvesting}

At the end of the incubation period, each platform was slowly lifted to the surface by the four corner chains and all 18 tiles were carefully removed (without touching the top of the tiles). Tiles for analysis were all randomly selected and all tiles were harvested within an hour of removing them from the pond (except for the final six incubators in the fall sampling). Tiles with large amounts of debris (e.g., leaves) or that were accidently touched during harvesting were replaced with another randomly-selected tile. Tiles were not enclosed in grazer-proof netting and were therefore, exposed to grazers throughout the experiment. To remove the effect of the FA contained in grazers on determining biofilm biomass and FA profile, all tiles were visually inspected (for more detail a magnifying glass was occasionally used) and any visible surface and 
sub-surface grazers (e.g., oligochaete and insect larvae) were removed with an ethanol-washed tweezer prior to harvesting. Most tiles were visually inspected for 3 - 5 min prior to being harvested, however, the abundance of grazers was not recorded in order to minimize the time necessary to harvest the tiles. Biofilm was scraped from a tile using a stainless-steel razor blade; washed, between scrapings, with ethanol. The biofilm from nine tiles was scraped and combined into three separate pre-weighed Falcon ${ }^{\circledR}$ tubes for triplicate representative samples of the incubator. All Falcon ${ }^{\circledR}$ tubes were immediately placed at $-20^{\circ} \mathrm{C}$ (in the freezer at the landowner's

residence) but were transferred on ice (vehicle transport, within $24 \mathrm{~h}$, back to lab) and where they were then stored at $-80^{\circ} \mathrm{C}$. One tile was cleaned (i.e., the bottom and side of the tile was scraped with a razor) prior to placing it in a plastic Tupperware ${ }^{\circledR}$ container filled with $\sim 500 \mathrm{~mL}$ of filtered (via $28 \mu \mathrm{m} \mathrm{Nitex}{ }^{\circledR}$ mesh) pond water; the tile was used for CLSM analysis.

\subsubsection{Confocal Laser Scanning Microscopy Analysis}

Tiles selected for CLSM analysis were stored in filtered pond water and at $4^{\circ} \mathrm{C}$ until analysis. A Nikon Eclipse 80i-C1 microscope (Nikon Instruments Inc., Melville, NY) and a $20 \times / 0.50$ NA Plan Fluor objective lens were used for CLSM analysis. Autofluorescence of attached biomass was visualized with a $632 \mathrm{~nm}$ excitation wavelength and a long pass $650 \mathrm{~nm}$ detector. For each tile, ten random microscope fields were chosen and the biofilm in each field was imaged by capturing stacks in the Z-direction, spanning the entire thickness of the biofilm. The step size between slices in the Z-stack was maintained at $0.8 \mu \mathrm{m}$ for all imaging. Images were acquired using the EZC1 software (Nikon Instruments Inc., Melville, NY), and acquired images were subsequently reconstructed into maximum intensity projections and threedimensional images using the NIS-elements software package (Nikon Instruments Inc., Melville, NY). Z-stack images were also analyzed via COMSTAT (Heydorn et al. 2000) in MatLab (The MathWorks, Natick, MA) to quantify the structural profile of the biofilm. All slices in each Zstack were first converted to greyscale tagged image format (TIF) files using IrfanView (Version 4.35, www.irfanview.com). Grey-scaled images were then subjected to COMSTAT analysis to determine structural parameters of the biofilm. Bio-volume $\left(\mu \mathrm{m}^{3} / \mu \mathrm{m}^{2}\right)$ is defined as the number of biomass pixels, multiplied by the size of the pixel, divided by the substratum area; this represents the overall wet volume of a biofilm. The highest point above each pixel determined biofilm thickness and was used to calculate the mean thickness $(\mu \mathrm{m})$ of a sample. The roughness 
coefficient (unitless) was calculated as the variance of all thickness measurements from the mean thickness. Reported values for each of the aforementioned parameters represent an average of the ten fields imaged for each tile.

\subsubsection{Lipid and Fatty Acid Analysis}

All pre-weighed Falcon ${ }^{\circledR}$ tubes containing biofilm samples were weighed to determine the wet weight (WW) of harvested biofilm. Samples were then freeze-dried for 3 days and measured immediately after to determine the DW of biofilm harvested. The content of two Falcon ${ }^{\circledR}$ tubes (i.e., six randomly selected tiles) was combined together and mixed well to ensure substantial biomass for analysis. The small subsample $(10-30 \mathrm{mg})$ of the mixed lyophilized samples were weighed in pre-weighed aluminum weighting boats and ashed in a muffler furnace at $450^{\circ} \mathrm{C}$ for $2 \mathrm{~h}$, then weighed again. The difference between the before and after weight of the sample represents the organic matter or the ash-free-dry weight (AFDW).

FA analysis was performed using a modified Folch (1957) method. Approximately 30 $50 \mathrm{mg}$ of lyophilized biomass was added to $4 \mathrm{~mL}$ of chloroform:methanol (2:1 by volume) to extract overnight at $-80^{\circ} \mathrm{C}$. An internal standard (23:0) was added to each sample prior to extraction. The following day, $\mathrm{KCl}(0.88 \%$ in water) was added, and samples were centrifuged (5 min, $2000 \mathrm{rpm}, 4^{\circ} \mathrm{C}$ ). All samples were kept on ice during extraction. The lower layer, containing lipids was transferred to a new tube. Lipids were extracted twice more with $2 \mathrm{~mL}$ theoretical lower phase (86:14:1 chloroform:methanol:water). Samples were evaporated under nitrogen gas and re-suspended in hexane. A small aliquot was transferred to tin cups for gravimetric determination of total lipids (expressed here as $\mu \mathrm{g}$ total lipids/mg AFDW). Samples were purged with nitrogen gas and trans-methylated in acidic conditions (1\% sulfuric acid) at $90^{\circ} \mathrm{C}$ for 90 mins (Indarti et al. 2005). Milli-Q water was added to stop the methylation reaction

and fatty acid methyl esters (FAME) were extracted three times with hexane. Samples were evaporated to dryness under nitrogen gas and a known volume of hexane was added with a digital analytical syringe (SGE; $\left.\mathrm{eVol}{ }^{\circledR} \mathrm{XR} ; 2910200\right)$ and transferred to vials for gas chromatography.

FAME were analyzed with a GC (Shimadzu GC-2010 plus) equipped with a flame ionization detector. A Supelco (SP-2560) column $(100 \mathrm{~m} \times 0.25 \mathrm{~mm} \times 0.20 \mu \mathrm{m})$ was used for 
separation of FAME. Helium was used as the carrier gas with an average flow of $5.2 \mathrm{~mL} \mathrm{~min}^{-1}$. A splitless injection $(1 \mu \mathrm{L})$ was used with the following temperature program: 1) initial temperature $60^{\circ} \mathrm{C}$ was maintained for $\left.1 \mathrm{~min}, 2\right)$ temperature was increased, at a rate of $10^{\circ} \mathrm{C}$ $\mathrm{min}^{-1}$, to $\left.180^{\circ} \mathrm{C}, 3\right)$ temperature was increased, at a rate of $2^{\circ} \mathrm{C} \min ^{-1}$, to $220^{\circ} \mathrm{C}$, and, 4) temperature was finally increased, at a rate of $5^{\circ} \mathrm{C} \min ^{-1}$, to $240^{\circ} \mathrm{C}$, which was then maintained for 23 min. A standard FAME mix, GLC-463 (Nu-Chek Prep.), was used for peak identification. An external standard, GLC-68E (Nu-Chek Prep.), was used for calibration curves to determine concentration of FAME (in $\mu \mathrm{g} / \mu \mathrm{L}$ ). FAME concentrations, final volume, and AFDW were used to calculate the mass of FAME per milligram of ash-free dry weight ( $\mu \mathrm{g}$ FAME/mg AFDW), the content of a FA response of each biofilm sample standardized for AFDW. The overall yield of a FA response was calculated by multiplying the standardized mass by the total AFDW obtained from six combined tiles and divided by the area $\left(\mathrm{in}^{2}\right.$ ). It is important to note that the FA "yield" of biofilm calculated in this study refers only to the FA content remaining on the tiles after the incubation period and does not account for FA removal by grazers, sloughing, and diffusion or FA addition by organic deposition. Thus, yield in this study should be thought of as an "instantaneous yield" (i.e., standing stock) rather than as a true estimate of biofilm productivity (i.e., a rate measurement) with respect to FA.

\subsubsection{Statistical Analysis}

Normality and heterogeneity of variance of all raw dependent variables were checked prior to analysis. In cases where raw data failed Shapiro-Wilk's normality test (Shapiro \& Wilk 1965), data were transformed using box-cox transformation (Box \& Cox 1964, 1981), a family of power transformations, so that data would then pass the Shapiro-Wilk's normality test to ensure dependent variables met the distributional assumptions of later statistical tests. All data sets, except for the temperature data from the iButtons, were composed of multiple dependent variables, and therefore, were first subject to a multivariate analysis of variance (MANOVA) to test the effect of independent variables and their interactions on the overall data set. In the YSI and CLSM data sets, each response variable was converted to Z-scores for MANOVA analysis to account for differences between units and scales. In cases of significant main or interaction effects $(\mathrm{p}<0.05)$, the data set was subject to an analysis of variance (ANOVA) test for each dependent variable. In all data sets, except light (where only season and depth and their 
interaction was tested) and lipid (failed unimodal test), the main effect of seasons, blocks, depths as well as the interaction of season $\times$ block, season $\times$ depth, and block $\times$ depth were examined. Although the color of the pool noodle was randomized between incubators, the main, and any interactive, effects of color were assumed to have little biological significance and were therefore not included in any models or analyses.

The YSI (i.e., water temperature, conductivity, TDS, pH, ODO, and CDOM), iButton (i.e., hourly water temperature), and CLSM (i.e., bio-volume, mean thickness, and roughness coefficient) datasets, as well as the DW and AFDW data, were analyzed in response to the independent variables and their interactions mentioned above. To remove the hourly effect of iButton water temperature data, a model composed of temperature in response to hour, as the only independent factor, was used to generate residuals. These values represent water temperature data without the influence of hourly measurements; these residuals were run as the response variable in the ANOVA model for the iButtons data set. For light measures, the area under the curve of raw irradiance $\left(\mu \mathrm{W} / \mathrm{cm}^{2} / \mathrm{nm}\right)$ values was calculated for PAR $(400-700 \mathrm{~nm})$, UV-R $(280 \mathrm{~nm}-400 \mathrm{~nm}), \mathrm{UV}-\mathrm{A}(320-400 \mathrm{~nm})$, and UV-B $(280-320 \mathrm{~nm})$ to analyze total absolute irradiance $\left(\mathrm{mW} / \mathrm{cm}^{2}\right)$ in the respective wavelength ranges.

The lipid data could not be transformed into normally-distributed data. A significant Hartigan's dip test statistic (Hartigan \& Hartigan 1985) for unimodality suggested non-unimodal data (i.e., at least bimodal). The R package "diptest” (Maechler 2015) was used to conduct the Hartigan's Dip Test. The data were then divided into two sets based on season; a Student's t-test was used to determine the effect of season on individual response variables. All responses in both data sets passed the unimodal test and were then transformed to satisfy the assumption of normality and analyzed, via ANOVA, to test the effect of depth and block. However, due to limited degrees of freedom the interaction was not tested. For all ANOVAs where significant differences were found, Tukey's HSD post-hoc tests were applied for multiple comparisons. To minimize the risk of Type I errors (i.e., false positives), a Šidák adjustment was used for confidence intervals and a Tukey adjustment was used for p-values.

The specific FA that were identified and captured by the summary indices are listed in Appendix 4. The unsaturation index was of a sample was calculated by multiplying the mass fraction of each FA ( $\mu \mathrm{g} / \mathrm{mg}$ AFDW) by the number of double bonds in the FA and summing 
them together for each sample. The level of significance for all analyses was set at $\alpha=0.05$ and all analyses were conducted using R Studio Desktop version 1.1.442 (RStudio Team, 2016). All graphs were generated using SigmaPlot 12.5 (Systat Software, San Jose, CA) and GraphPad Prism 7 (GraphPad Software, La Jolla California USA). The "lsmeans" R package was used to assign letters between statistically-significant groups (Lenth 2016). All data reported in text are reported as mean $\pm \mathrm{SD}$. 


\section{$\underline{3.3 \text { Results }}$}

\subsubsection{Abiotic Factors}

Not surprisingly, summer water temperatures $\left(22.3 \pm 2.1^{\circ} \mathrm{C}\right)$ were warmer than fall $(8.9 \pm$ $4.6^{\circ} \mathrm{C}$ ) water temperatures (ANOVA, $\mathrm{p}<0.0001$; Table A5.1). Overall, water temperatures decreased with increasing depths (Tukey HSD, p < 0.0001), however, the effect was only observed in the summer months (Fig. 3.3). Block $\mathrm{C}$ had higher mean water temperatures than both Block A and B (Tukey HSD, p < 0.0001), but this spatial variability was also only observed in the summer months and only at certain depths $(25,100$, and $125 \mathrm{~cm}$; Tukey HSD, p < 0.05).

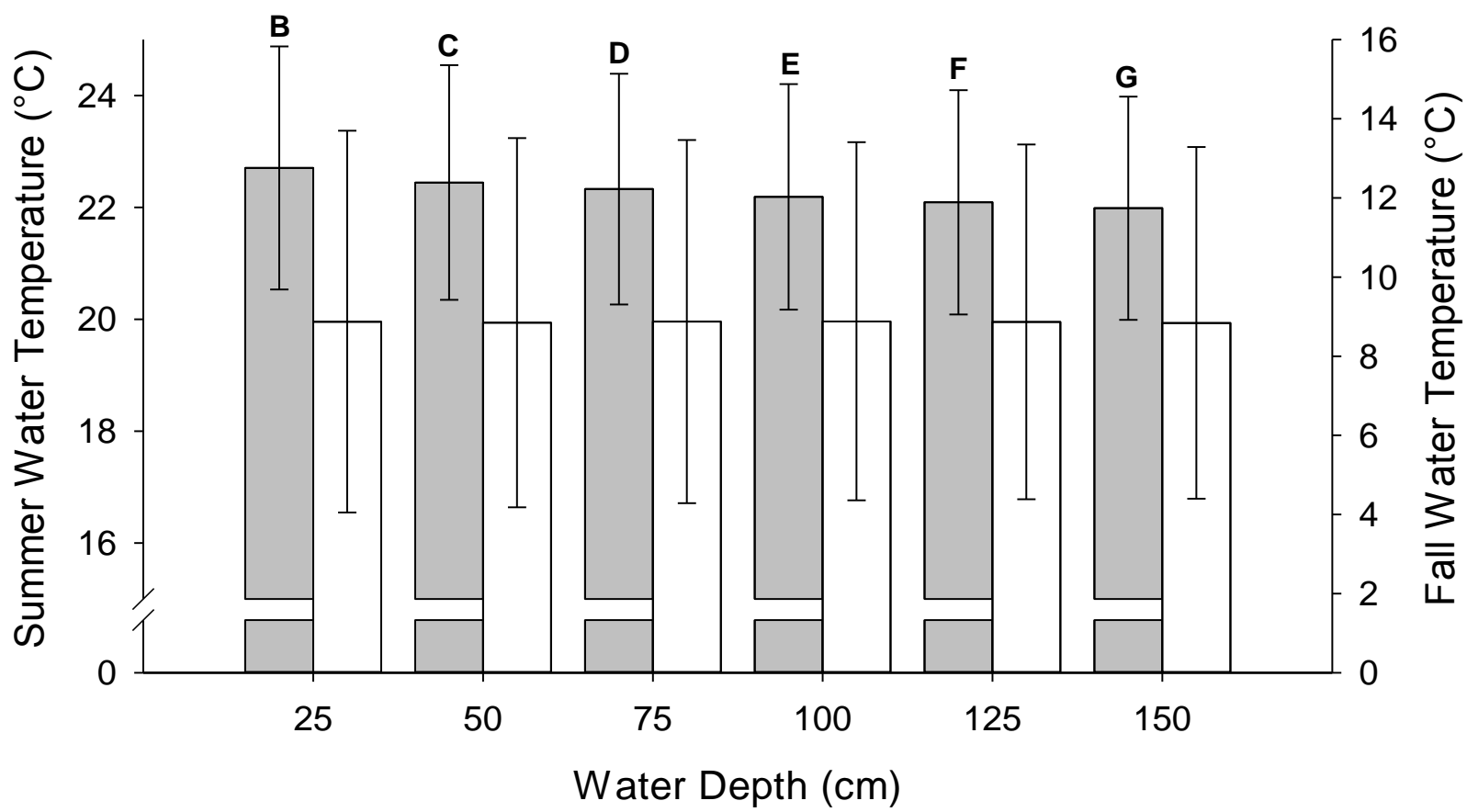

Figure 3.3: Seasonality of depth-effect differences in water temperature $\left({ }^{\circ} \mathrm{C}\right.$ ) in summer (grey fill - left y-axis) and fall (white fill - right y-axis). Groups marked with different letters are significantly different (Tukey HSD post-hoc test using the season $\times$ depth interaction term). No differences were observed between depths in fall, therefore, the letter ("A") above the bars was removed for clarity purposes.

Weekly YSI measurements (temperature, conductivity, TDS, pH, ODO, CDOM) demonstrated the strong influence of season and depth (and their interaction) on abiotic factors throughout the experiment (MANOVA, $\mathrm{p}<0.0001$; Table A5.2A). Similar to iButton data, season strongly influenced overall water temperature (ANOVA, $\mathrm{p}<0.0001$; Table A5.2B) as mean summer temperatures were $21.2 \pm 1.9^{\circ} \mathrm{C}$ and fall temperatures were $7.0 \pm 3.5^{\circ} \mathrm{C}$. Season 
also strongly influenced conductivity, TDS, pH, ODO, CDOM (ANOVA, p < 0.0001; Table A5.2C-G). Dissolved oxygen and $\mathrm{pH}$ were higher in the summer season; conductivity, TDS, and CDOM, were all higher in the fall season (Table A5.2). Overall, depth also strongly influenced CDOM measurements (ANOVA, p < 0.0001; Table A5.2G), which increased gradually with depth, but only in the summer season (Tukey HSD, p < 0.05).

Season and depth and their interaction had a strong influence on the underwater light climate (MANOVA, p < 0.0001; Table A5.3A). Overall, summer had higher PAR (400 - 700 $\mathrm{nm})$ irradiance $\left(15.66 \pm 5.88 \mathrm{~mW} / \mathrm{cm}^{2}\right)$ than fall $\left(6.17 \pm 3.69 \mathrm{~mW} / \mathrm{cm}^{2}\right.$; ANOVA, $\mathrm{p}<0.0001$; Table A5.3B). PAR also decreased with depth and this relationship was observed in both seasons (Fig. 3.4). Higher UV-R (280 - $400 \mathrm{~nm})$ levels were observed in summer $\left(0.50 \pm 0.43 \mathrm{~mW} / \mathrm{cm}^{2}\right)$ than fall $\left(0.23 \pm 0.20 \mathrm{~mW} / \mathrm{cm}^{2}\right.$; ANOVA, $\mathrm{p}<0.0001$; Table A5.3B). In both seasons, UV-R did decrease with increasing depth (Fig. 3.5AC). Both season and depth had a strong influence on UV-A $(320-400 \mathrm{~nm})$ and UV-B $(280-320 \mathrm{~nm})$ as, in both cases, higher levels of UV radiation were observed generally in the summer and at shallower depths (Fig. 3.5B and C).

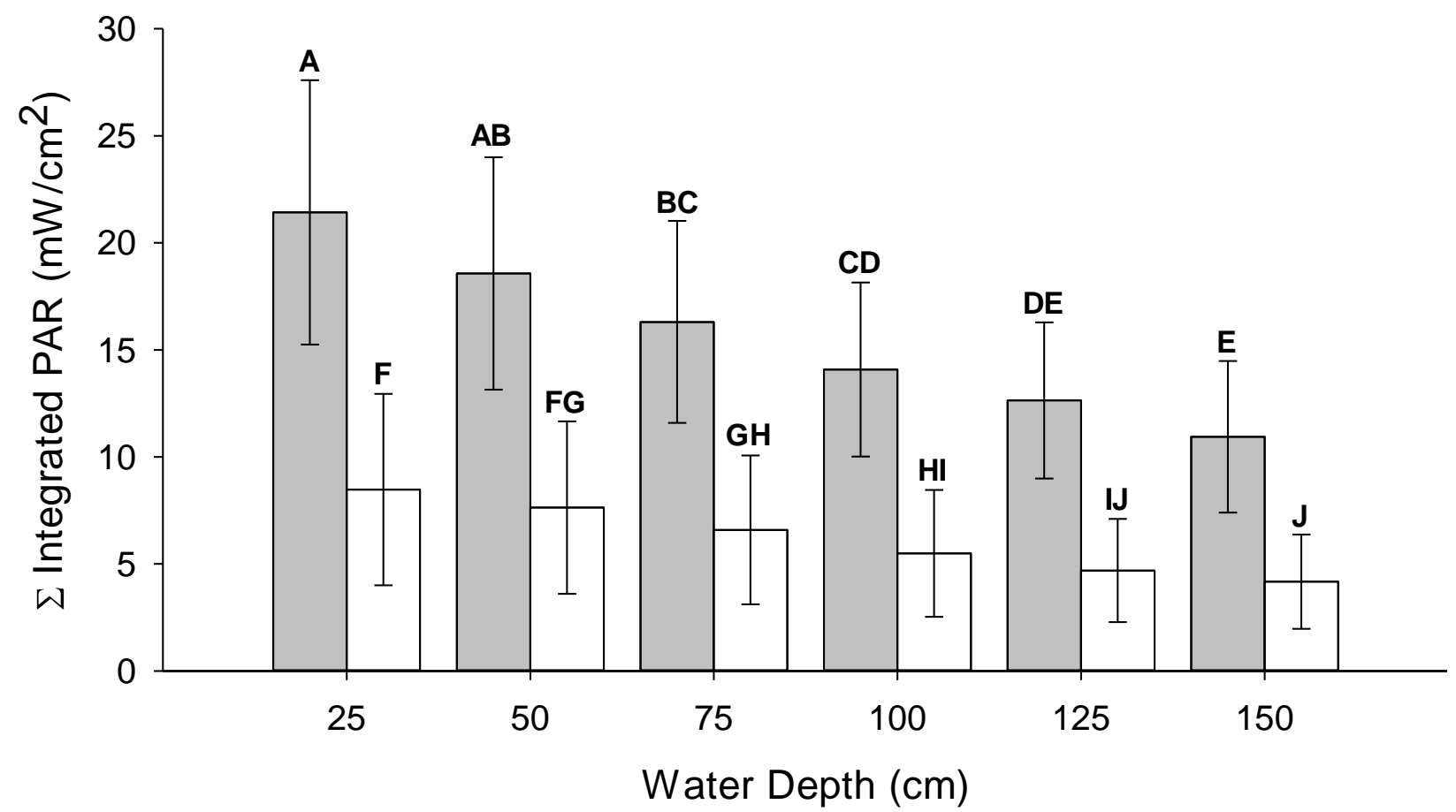

Figure 3.4: Seasonality of depth-related difference in PAR irradiance $\left(\mathrm{mW} / \mathrm{cm}^{2}\right)$ in summer (grey fill) and fall (white fill). Groups marked with different letters are significantly different (Tukey HSD post-hoc test using the season $\times$ depth interaction term). 

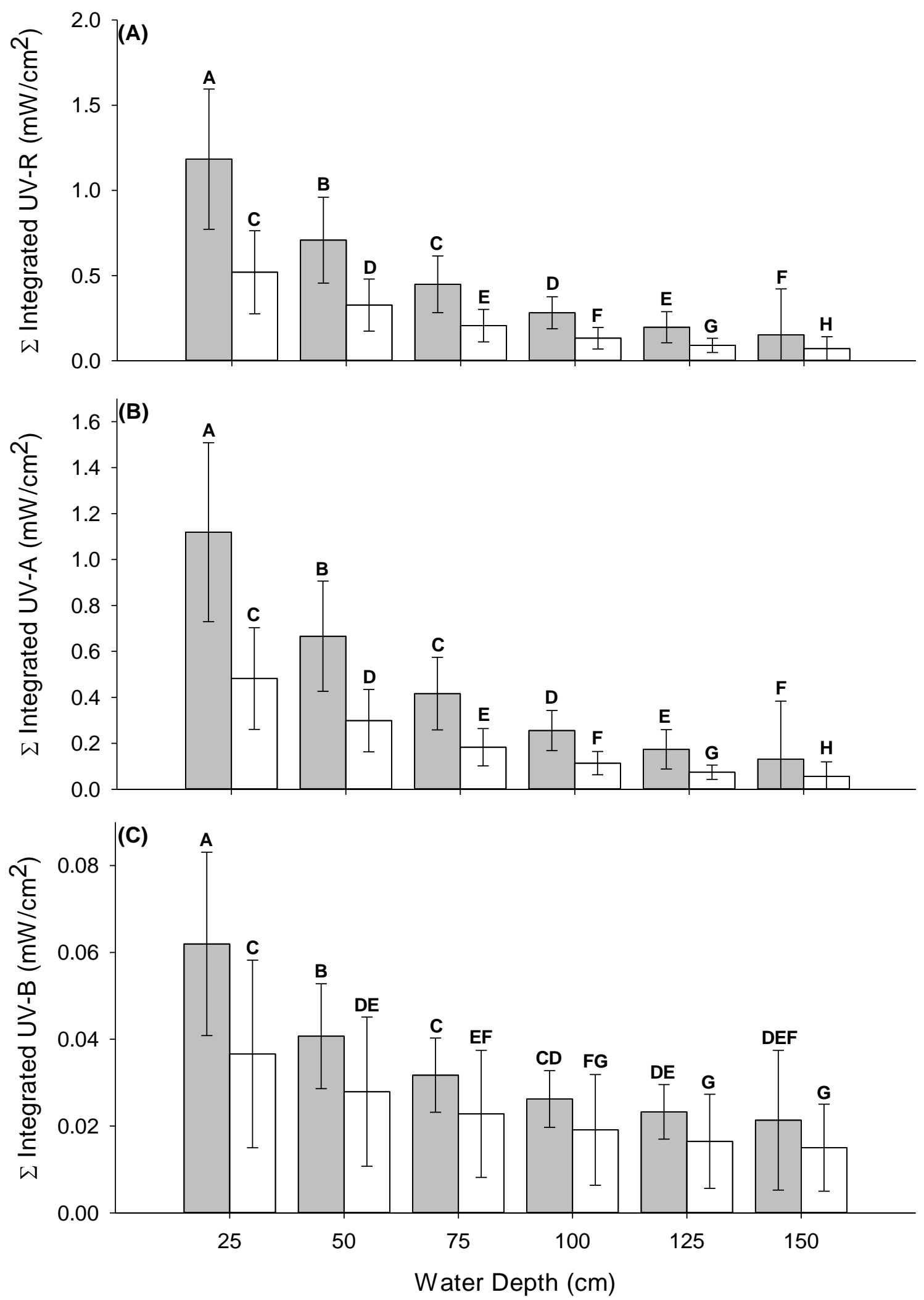

Figure 3.5: Seasonality of depth-related difference in (A) UV-R, (B) UV-A, and (C) UV-B irradiance $\left(\mathrm{mW} / \mathrm{cm}^{2}\right)$ in summer (grey fill) and fall (white fill). Groups marked with different letters are significantly different (Tukey HSD post-hoc test of the season $\times$ depth interaction). 


\subsubsection{Confocal Laser Scanning Microscopy Analysis}

Depth, season, block, and all interactions between them had highly significant effects on the overall structural profile of biofilm (MANOVA, $\mathrm{p}<0.0001$; Table A5.4A). Depth, block, and all interactions significantly affected the biofilm bio-volume (ANOVA, $\mathrm{p}<0.05$; Table A5.4B), however, season did not (ANOVA, p > 0.05; Table A5.4B). Overall, deeper tiles had a higher bio-volume and this trend is seen in both summer and fall biofilm (Tukey HSD, $\mathrm{p}<0.05$; Fig. 3.6). In general, Block $C$ had lower bio-volume compared to both Block A and B (Tukey HSD, $p<0.01$ ), however, this trend only appeared in summer (Tukey HSD; $p<0.05$ ) and the block effect varied between depths (Tukey HSD).

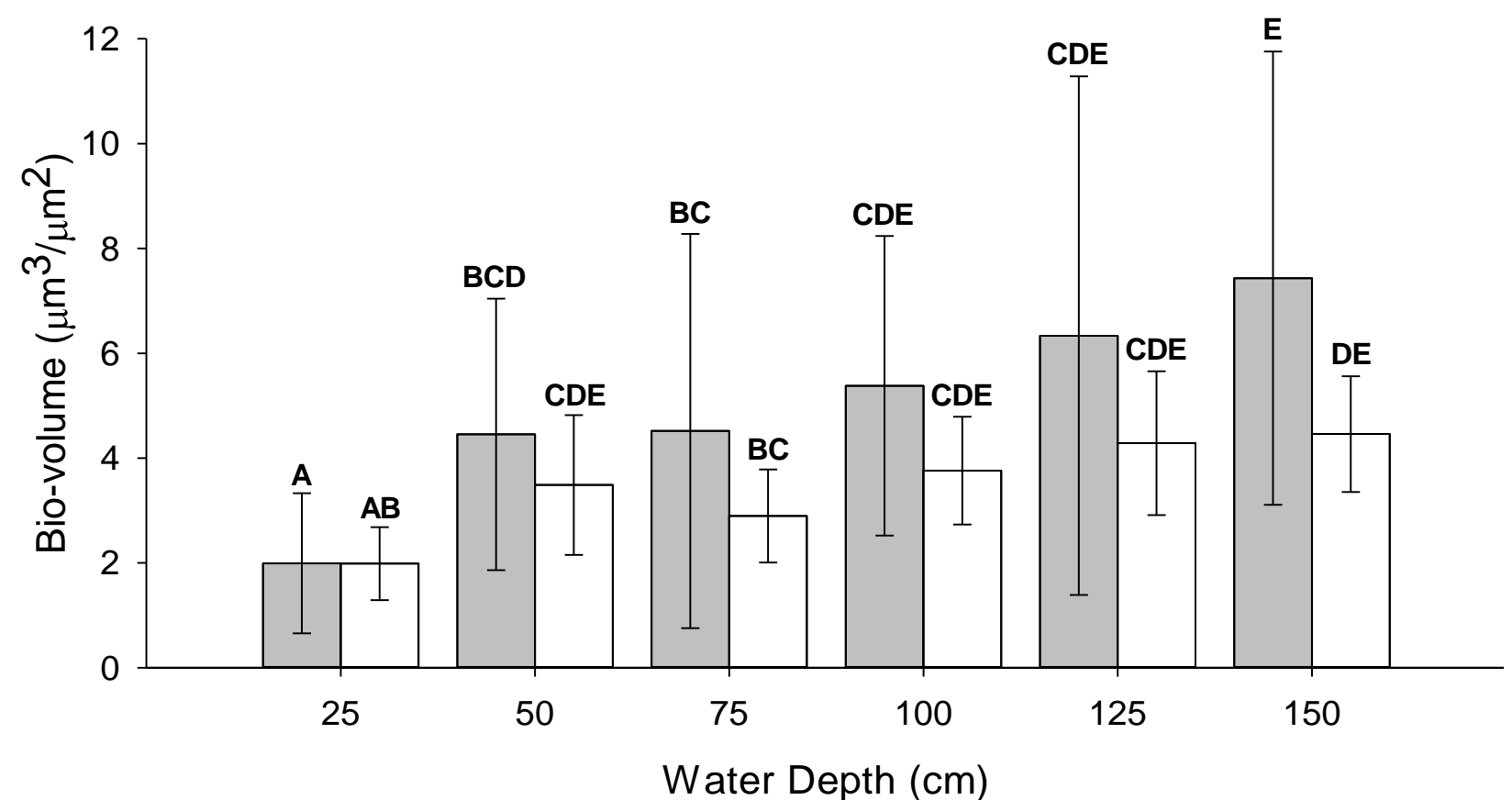

Figure 3.6: The effect of depth on biofilm bio-volume $\left(\mu \mathrm{m}^{3} / \mu \mathrm{m}^{2}\right)$ in summer and fall in summer (grey fill) and fall (white fill). Groups marked with different letters are significantly different (Tukey HSD post-hoc test using the season $\times$ depth interaction term).

All independent factors (season, depth, and block) and most interactions had an effect on the mean thickness of biofilm (ANOVA, $p<0.05$; Table A5.4C). Overall, summer biofilm had lower mean thickness compared to fall biofilm as the mean thickness of summer and fall biofilm was $33.6 \pm 17.6 \mu \mathrm{m}$ versus $37.8 \pm 13.3 \mu \mathrm{m}$, respectively (ANOVA, $\mathrm{p}<0.0001$, Table A5.4C). In both seasons, biofilm incubated at greater depths was, on average, thicker than biofilm incubated at shallower depths (Fig. 3.7). A significant interaction was observed between block and depth in mean thickness, however, differences varied between depths (Tukey HSD). Overall, Block B 
$(39.3 \pm 16.8 \mu \mathrm{m})$ had higher mean thickness than Block A $(33.2 \pm 14.4 \mu \mathrm{m})$ and marginally higher than $\mathrm{C}(34.6 \pm 15.4 \mu \mathrm{m}$; Tukey HSD, $\mathrm{p}<0.01$ and $\mathrm{p}=0.060$, respectively). This blockrelated effect can be attributed to differences in both seasons (Tukey HSD; $p<0.05$; Fig. 3.8).

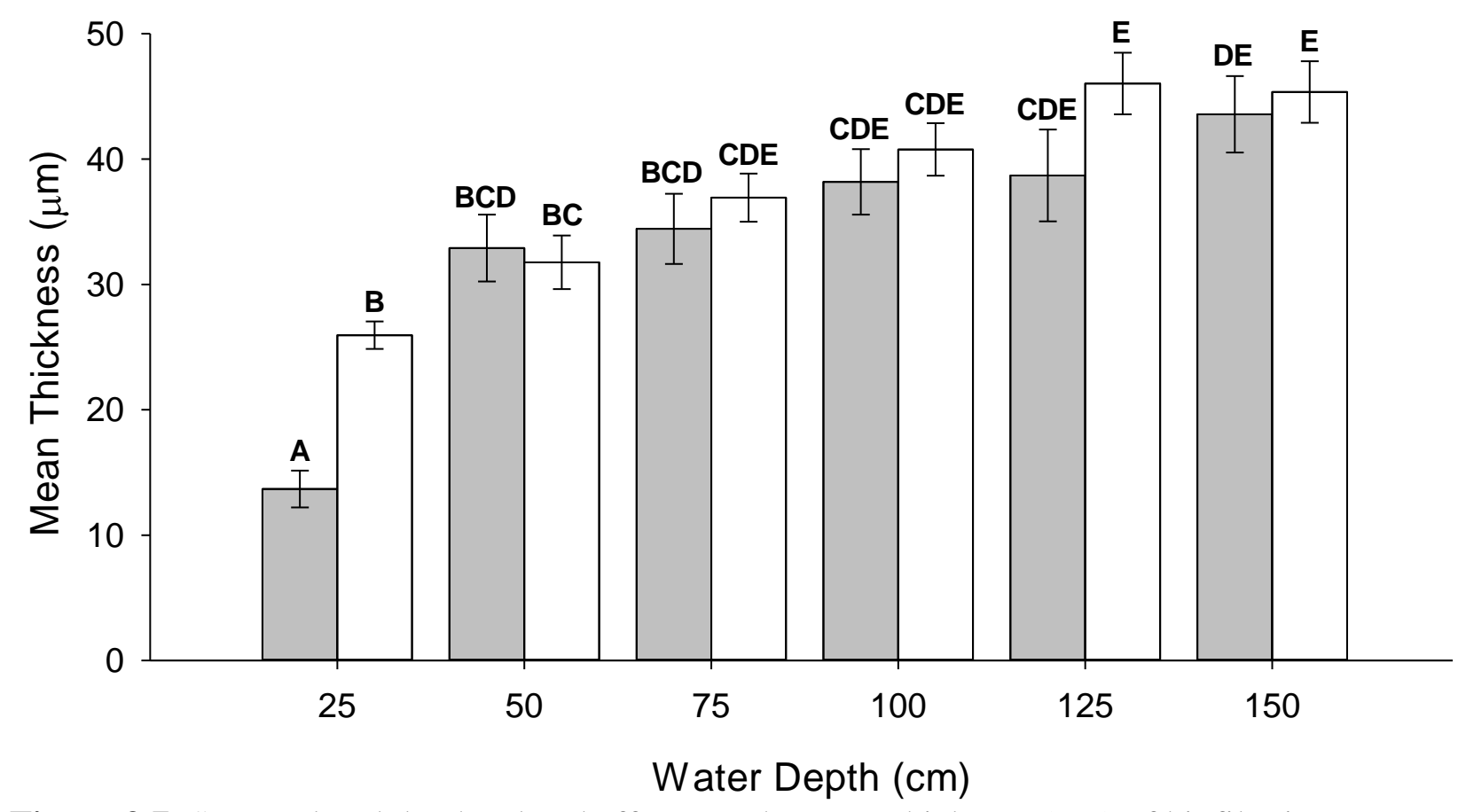

Figure 3.7: Seasonal and depth-related effects on the mean thickness $(\mu \mathrm{m})$ of biofilm in summer (grey fill) and fall (white fill). Groups marked with different letters are significantly different (Tukey HSD post-hoc test using the season $\times$ depth interaction term).

Overall, season did not influence the spatial heterogeneity of the biofilm on a given tile, indicated by the roughness coefficient (ANOVA, p > 0.05; Table A5.4D), however, there was a depth and block effect (ANOVA, $\mathrm{p}<0.0001$; Table A5.4D). Tile spatial heterogeneity decreased with depth (Tukey HSD, p < 0.05), decreasing gradually from $1.56 \pm 0.16$ at $25 \mathrm{~cm}$ (i.e., from near the surface) to $1.12 \pm 0.20$ at $150 \mathrm{~cm}$ from the surface. Overall, Block $\mathrm{C}(1.35 \pm 0.22)$ had higher spatial heterogeneity than both Block A (1.26 \pm 0.24$)$ and B (1.26 \pm 0.26$)$, however, this effect was driven only by differences summer biofilm (Tukey HSD, $\mathrm{p}<0.0001$ ). 


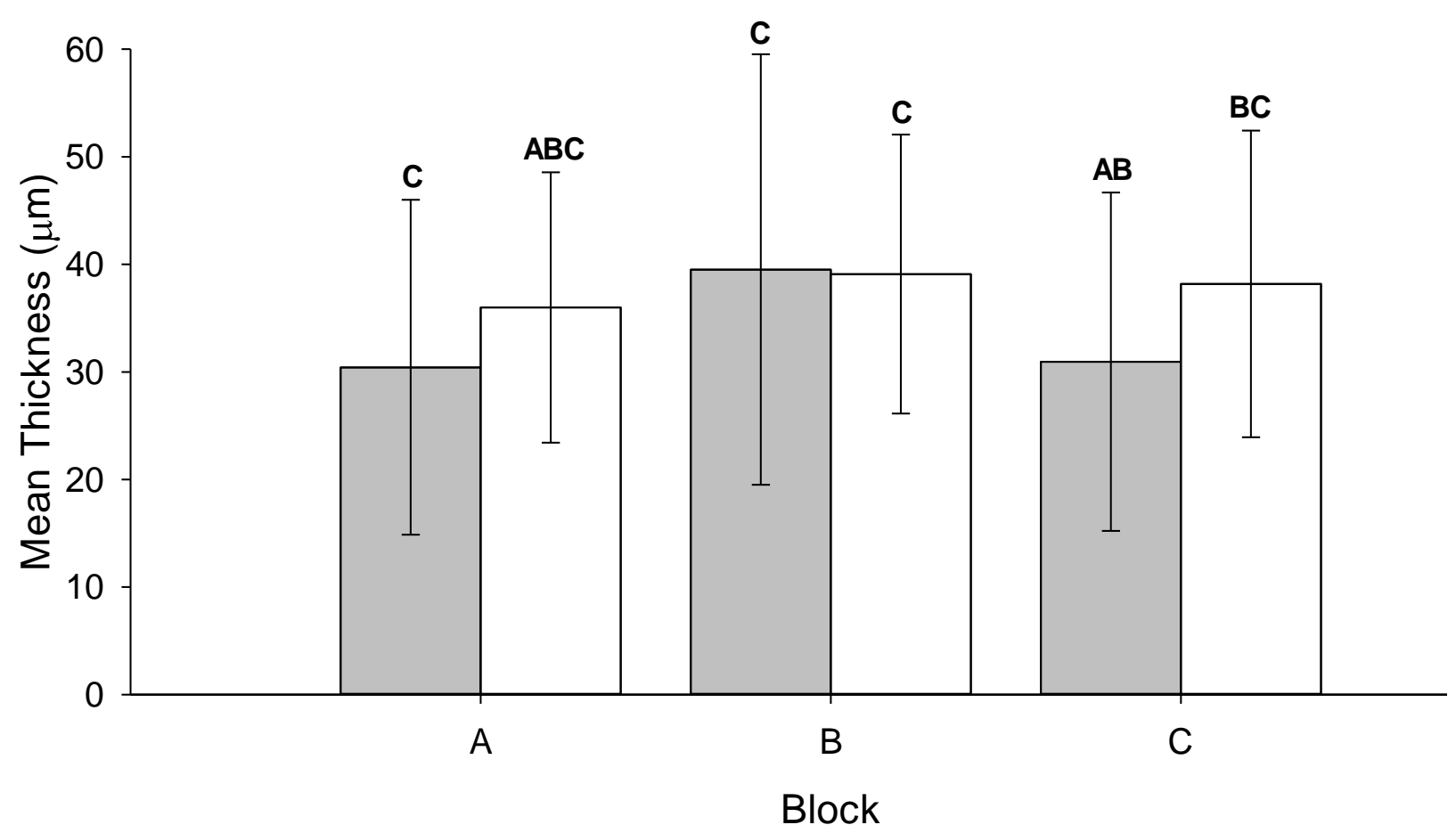

Figure 3.8: Seasonal effect of spatial variability in biofilm mean thickness $(\mu \mathrm{m})$ in summer (grey fill) and fall (white fill). Groups marked with different letters are significantly different (Tukey HSD post-hoc test using the season $\times$ depth interaction term).

\subsubsection{Dry Weights Analysis}

Overall, season, depth, and block had a strong influence on the DW of biofilm (ANOVA, $\mathrm{p}<0.0001$; Table A5.5A). The DW of biofilm collected on summer tiles $\left(9701 \pm 5592 \mathrm{mg} / \mathrm{m}^{2}\right)$ was higher than the DW of biofilm collected on fall tiles $\left(1916 \pm 235 \mathrm{mg} / \mathrm{m}^{2}\right.$; ANOVA, $\mathrm{p}<$ 0.0001; Table A5.5A). Fall biofilm ( $98.3 \pm 0.2 \%$ ) also had a higher moisture content than summer biofilm ( $94.2 \pm 1.9$; ANOVA, $\mathrm{p}<0.0001$; Table A5.5B). Overall, the dry weight increased with depth, however, this depth-related effect was also only observed in summer months (Fig. 3.9). Summer biofilm incubated at 25 and $50 \mathrm{~cm}$ had higher moisture content than the biofilm incubated at deeper depths (Tukey HSD, $\mathrm{p}<0.05$ ). This depth-related effect on moisture content was not observed in biofilm incubated during the fall (Tukey HSD, $p>0.05$ ). Block also affected the dry weight of biofilm collected, however, the effect was only observed in the summer (Fig. 3.10). No overall block effect was observed in the moisture content of biofilm (ANOVA, $\mathrm{p}>0.05$; Table A5.5B), however, Block C had lower moisture content than Block A and $B$ in summer biofilm (Tukey HSD, $p<0.01$ ). 


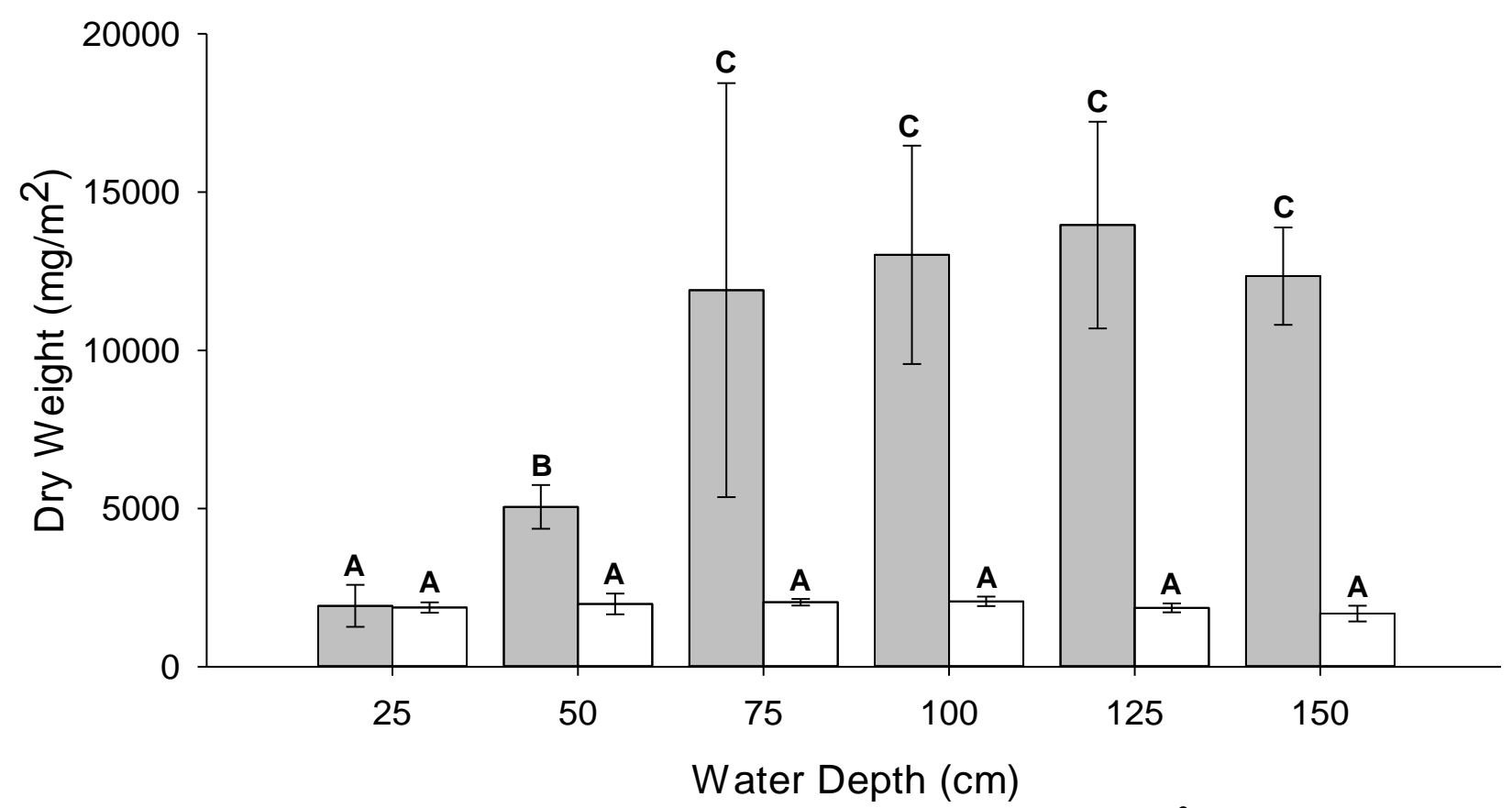

Figure 3.9: Seasonality of depth-related effect on biofilm dry weight $\left(\mathrm{mg} / \mathrm{m}^{2}\right)$ in summer (grey fill) and fall (white fill). Groups marked with different letters are significantly different (Tukey HSD post-hoc test using the season $\times$ depth interaction term).

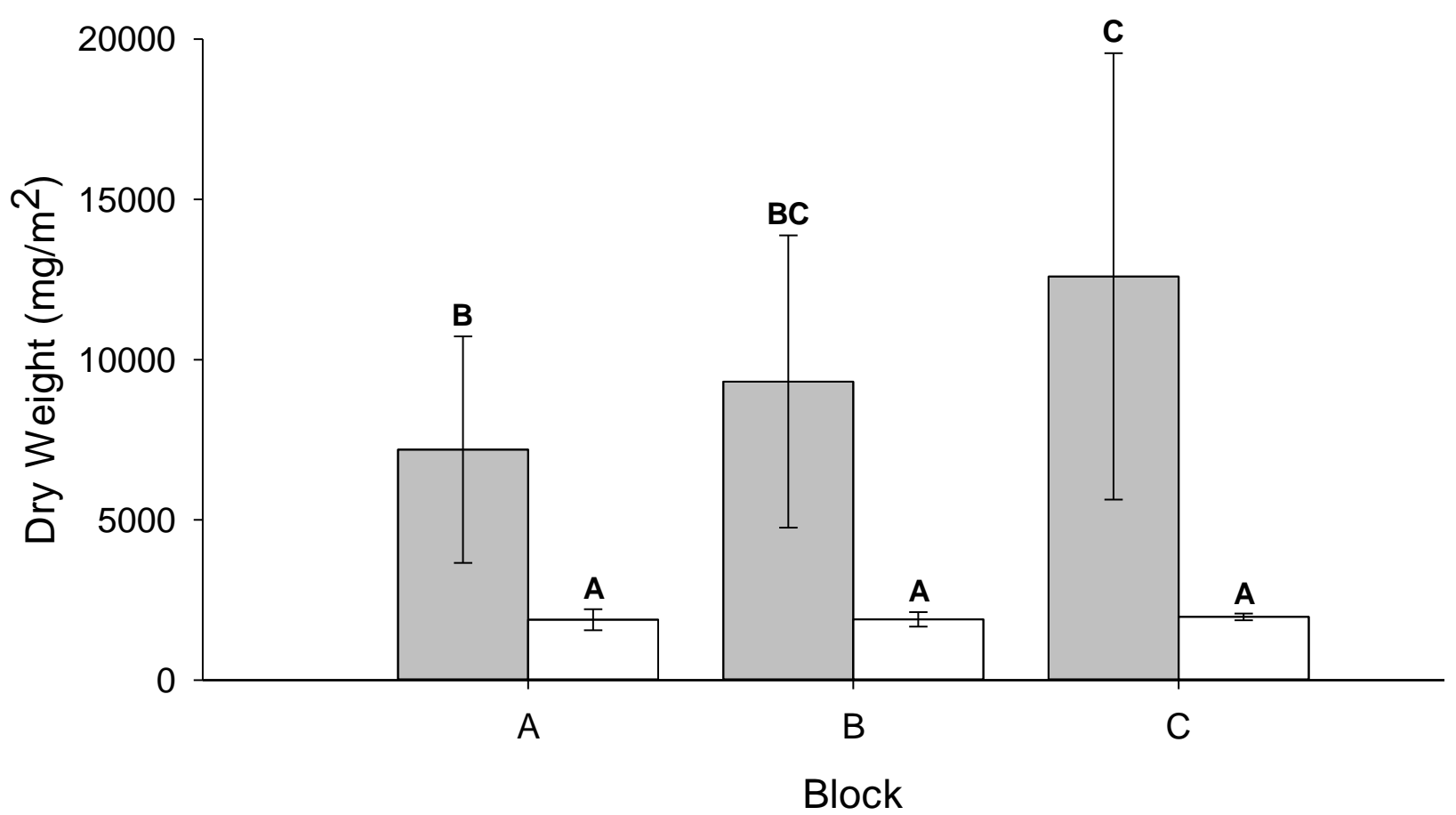

Figure 3.10: The spatial variability of biofilm dry weight $\left(\mathrm{mg} / \mathrm{m}^{2}\right)$ in summer (grey fill) and fall (white fill). Groups marked with different letters are significantly different (Tukey HSD post-hoc test using the season $\times$ depth interaction term). 


\subsubsection{Ash-free Dry Weight Analysis}

Season, depth, and their interaction had a significant effect on the AFDW of biofilm (ANOVA, $\mathrm{p}<0.0001$; Table A5.5C). Similar to the DW, the AFDW of biofilm collected on summer tiles $\left(3075 \pm 1457 \mathrm{mg} / \mathrm{m}^{2}\right)$ was higher than fall tiles $\left(907 \pm 117 \mathrm{mg} / \mathrm{m}^{2}\right.$; ANOVA, $\mathrm{p}<$ 0.0001; Table A5.5C). Overall, the AFDW also increased with depth, however, this depth-related effect was only observed in summer months (Fig. 3.11). The percentage of organic matter (i.e., percentage lost during ashing) was higher in fall $(46.8 \pm 2.9 \%)$ than summer $(34.3 \pm 5.6 \%)$ biofilm (ANOVA, $\mathrm{p}<0.0001$; Table A5.5D). There were no differences between depths (ANOVA, $p>0.05$; Table A5.5D), however, a significant season $\times$ depth interaction (ANOVA, $\mathrm{p}<0.0001$ ) demonstrated a summer-only decrease in organic content with depth (Fig. 3.12). Spatial variability was observed overall and interacting with season (Table A5.5D). Biofilm collected from Block $\mathrm{C}$ had a lower organic matter percentage than Block A and B in summer and Block A, but not B in fall (Fig. 3.13).

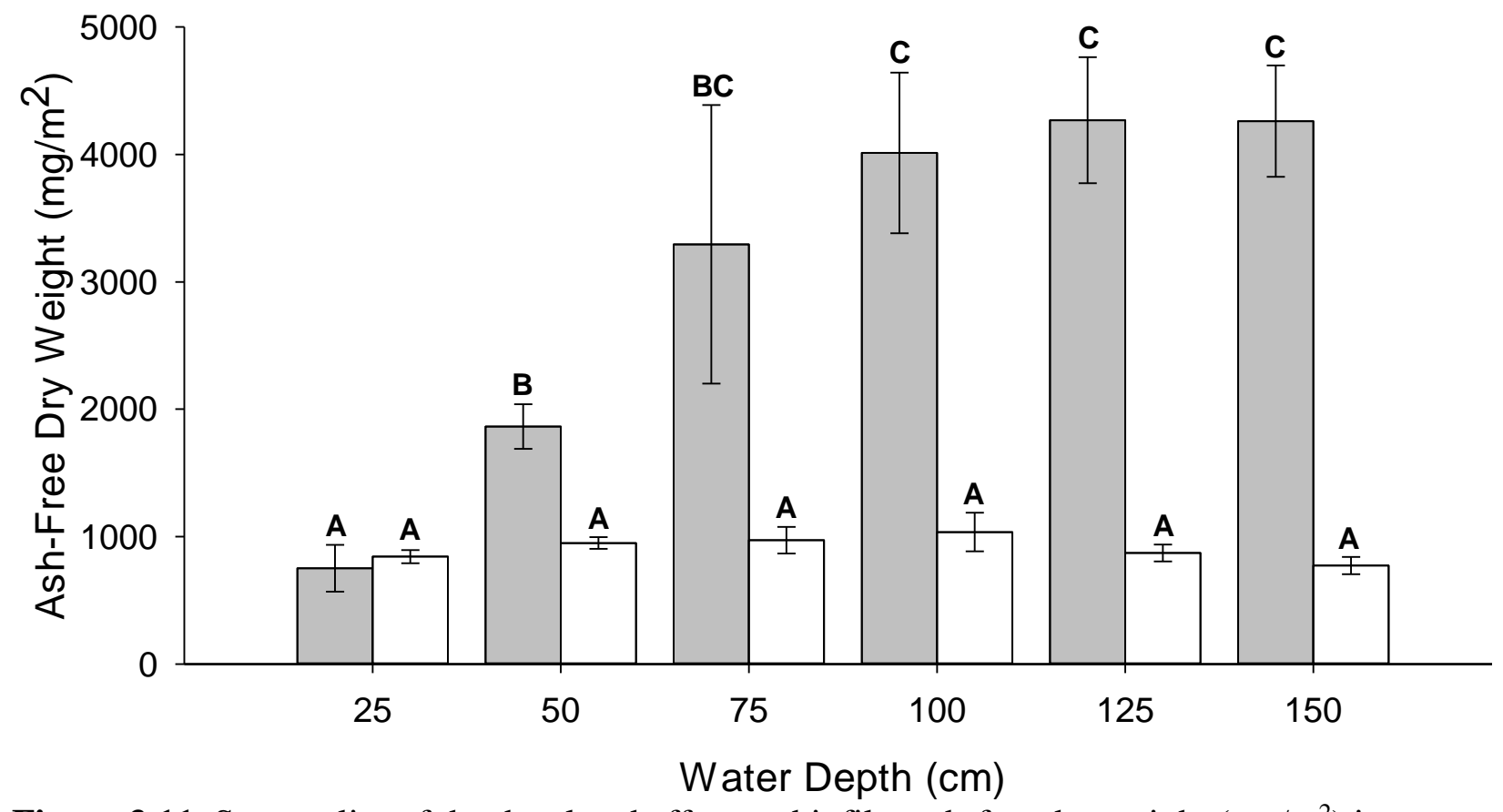

Figure 3.11. Seasonality of depth-related effect on biofilm ash-free dry weight $\left(\mathrm{mg} / \mathrm{m}^{2}\right)$ in summer (grey fill) and fall (white fill). Groups marked with different letters are significantly different (Tukey HSD post-hoc test using the season $\times$ depth interaction term). 


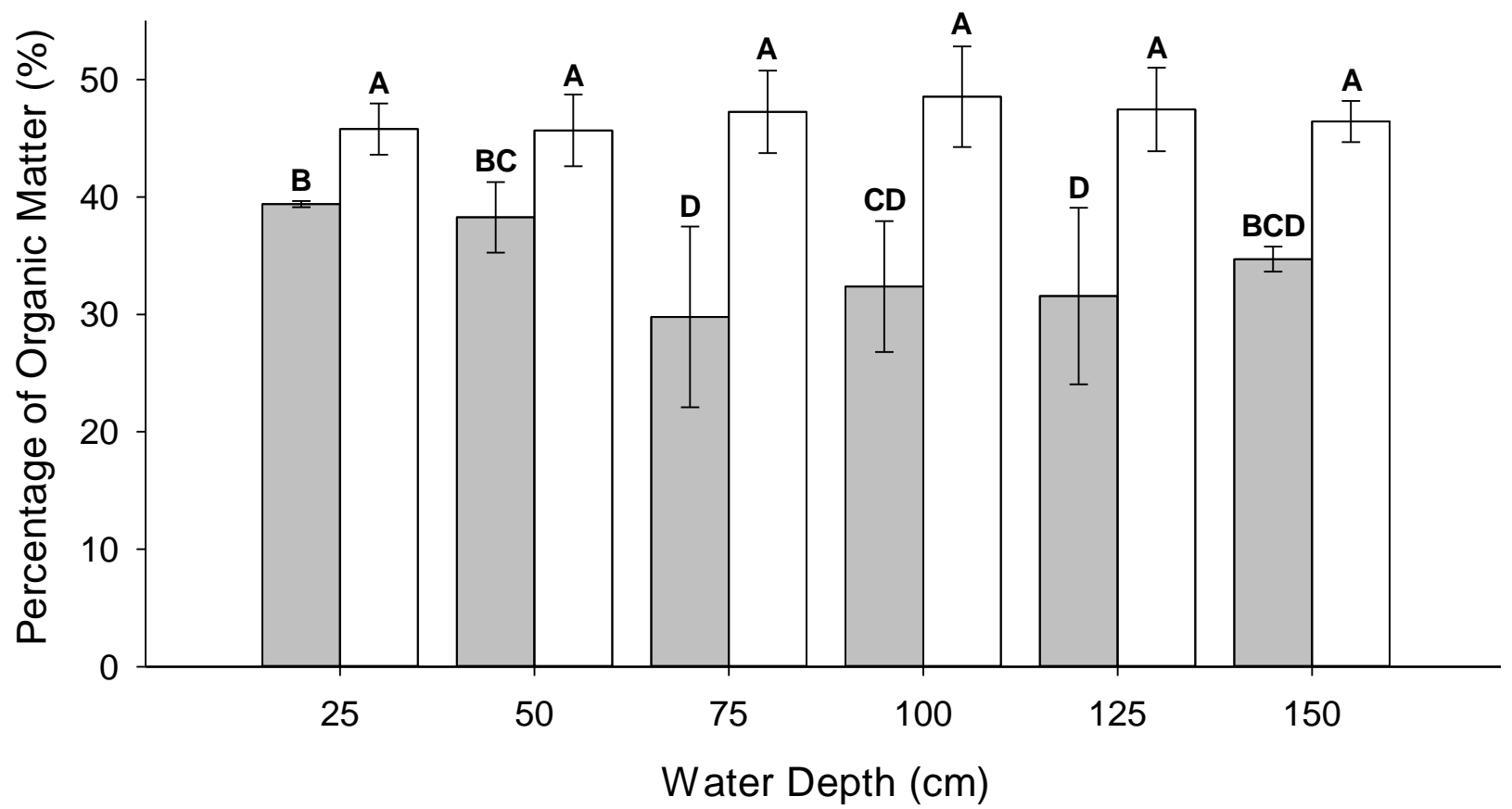

Figure 3.12. Seasonality of depth-related effect on the percentage organic matter of biofilm collected in summer (grey fill) and fall (white fill). Groups marked with different letters are significantly different (Tukey HSD post-hoc test using the season $\times$ depth interaction term).

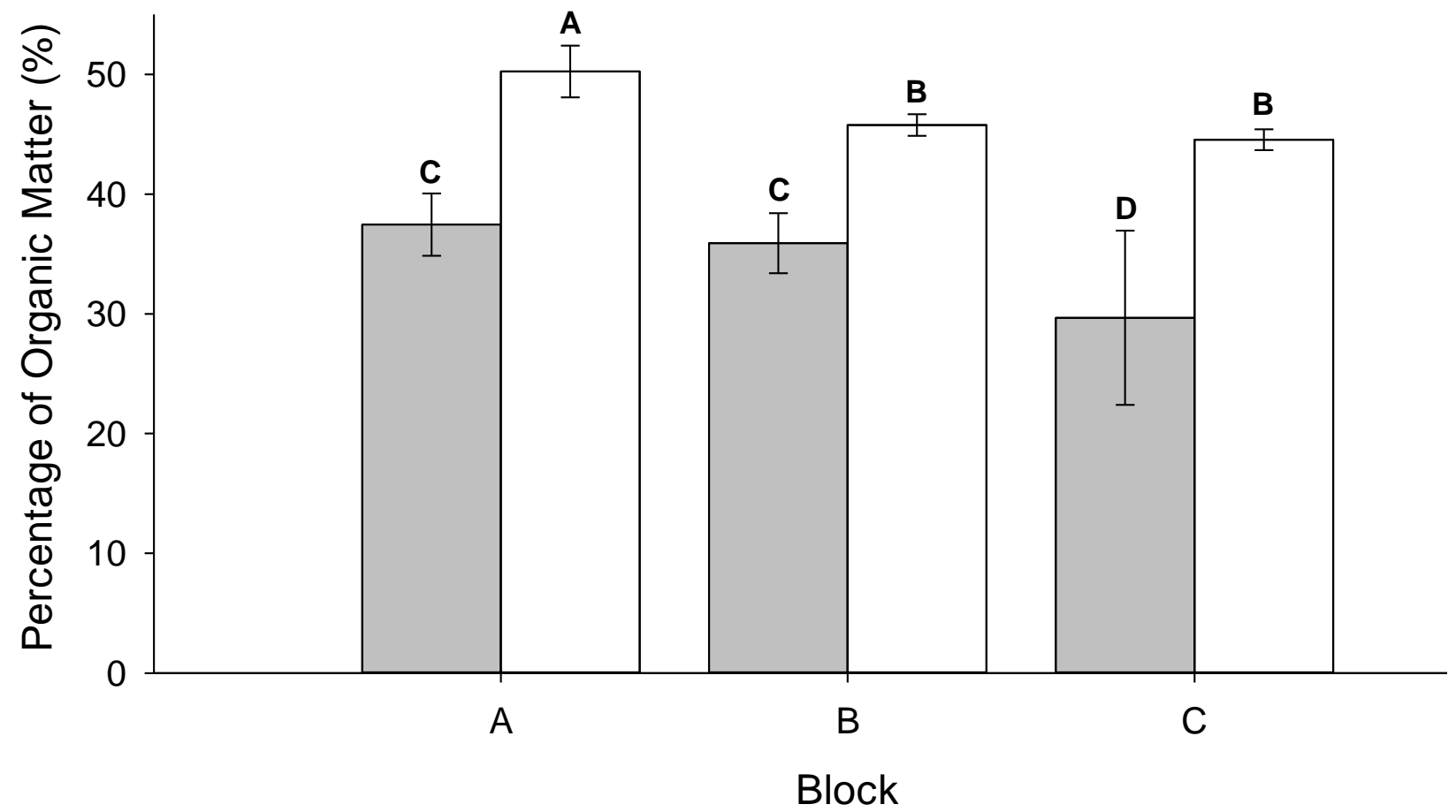

Figure 3.13. Seasonality of block-related effect on the percentage organic matter of biofilm collected in summer (grey fill) and fall (white fill). Groups marked with different letters are significantly different (Tukey HSD post-hoc test using the season $\times$ depth interaction term). 


\subsubsection{Fatty Acid Analysis}

Most responses in the lipid data set were significant $(\mathrm{p}<0.05)$ for a Hartigans' dip test for unimodality/multimodality, suggesting that these variables were not unimodally distributed and therefore that there were distinct subgroups (Table 3.1). Season had a highly significant effect on all FA responses analyzed (Table 3.1), and when the data set was separated by season all responses passed the unimodality test (Hartigans' dip test, $\mathrm{p}>0.05$ ). Total n-3 PUFA, n-6 PUFA, and EPA+DHA were used as indicators of nutritional value of biofilm. Fall biofilm had higher $\sum$ n-3 PUFA, $\sum$ n-6 PUFA and EPA+DHA content than summer biofilm, when standardized for differences in AFDW (Table 3.1). However, the total yield $\left(m g / m^{2}\right)$ of $\sum n-3$ PUFA, $\sum n-6$ PUFA and EPA+DHA was higher in the summer months (Student's t-test, $\mathrm{p}<0.001,0.0001$, and 0.01 respectively; Table 3.2).

Table 3.1: Average values for fatty acid responses from biofilm collected in summer and fall. Values are presented as mean $\pm \mathrm{SD}$. Absolute $\mathrm{t}$-values and $\mathrm{p}$-values were determined using a student's t-test after data were separated by season (to remove bimodal distribution). Parameters marked with $\mathrm{a} *$ and ${ }^{\dagger}$ represent response variables that were significant and marginally significant, respectively, for the Hartigans' dip test for unimodality/multimodality (suggesting data were not unimodal). Bolded p-values represent significance.

\begin{tabular}{|c|c|c|c|}
\hline Response & Summer & Fall & $\mathrm{t}$-value ( $\mathrm{p}$ value) \\
\hline \multicolumn{4}{|l|}{ Nutritional Quality } \\
\hline$\sum \mathrm{n}-3$ PUFA $(\mu \mathrm{g} / \mathrm{mg}$ AFDW) * & $7.0 \pm 1.2$ & $14.4 \pm 0.8$ & $22.13(<\mathbf{0 . 0 0 0 1})$ \\
\hline$\sum \mathrm{n}-6$ PUFA $(\mu \mathrm{g} / \mathrm{mg}$ AFDW $) *$ & $2.7 \pm 0.2$ & $2.9 \pm 0.3$ & $2.08(\mathbf{0 . 0 2 3})$ \\
\hline EPA+DHA $(\mu \mathrm{g} / \mathrm{mg} \text { AFDW })^{*}$ & $3.9 \pm 1.0$ & $8.5 \pm 0.7$ & $16.78(<\mathbf{0 . 0 0 0 1})$ \\
\hline \multicolumn{4}{|l|}{ Biomarkers } \\
\hline $16: 1 \mathrm{n}-7(\mu \mathrm{g} / \mathrm{mg} \text { AFDW })^{\dagger}$ & $6.6 \pm 1.1$ & $12.5 \pm 1.5$ & $13.77(<\mathbf{0 . 0 0 0 1})$ \\
\hline$\sum$ Odd-chained $(\mu \mathrm{g} / \mathrm{mg}$ AFDW $)$ & $0.5 \pm 0.03$ & $0.70 \pm 0.1$ & $9.47(<\mathbf{0 . 0 0 0 1})$ \\
\hline \multicolumn{4}{|l|}{ Summary Indices } \\
\hline$\sum$ FA $(\mu \mathrm{g} / \mathrm{mg} \text { AFDW })^{*}$ & $30.7 \pm 3.3$ & $49.6 \pm 4.2$ & $14.95(<\mathbf{0 . 0 0 0 1})$ \\
\hline$\sum \mathrm{SFA}(\mu \mathrm{g} / \mathrm{mg}$ AFDW $)$ & $10.6 \pm 0.9$ & $13.4 \pm 1.3$ & $7.60(<\mathbf{0 . 0 0 0 1})$ \\
\hline$\sum$ MUFA $(\mu \mathrm{g} / \mathrm{mg} \text { AFDW })^{*}$ & $10.3 \pm 1.2$ & $18.8 \pm 2.0$ & $15.37(<\mathbf{0 . 0 0 0 1})$ \\
\hline$\sum$ PUFA $(\mu \mathrm{g} / \mathrm{mg} \text { AFDW })^{*}$ & $9.7 \pm 1.3$ & $17.3 \pm 1.1$ & $18.82(<\mathbf{0 . 0 0 0 1})$ \\
\hline SFA $\% *$ & $34.7 \pm 1.0$ & $27.1 \pm 0.5$ & $29.99(<\mathbf{0 . 0 0 0 1})$ \\
\hline MUFA \% & $33.7 \pm 1.1$ & $37.8 \pm 1.1$ & $11.19(<\mathbf{0 . 0 0 0 1})$ \\
\hline PUFA \% & $31.6 \pm 1.6$ & $35.1 \pm 1.3$ & $6.95(<\mathbf{0 . 0 0 0 1})$ \\
\hline $18: 1 \mathrm{n}-9(\mu \mathrm{g} / \mathrm{mg} \text { AFDW })^{*}$ & $1.6 \pm 0.1$ & $4.4 \pm 0.6$ & $18.78(<\mathbf{0 . 0 0 0 1})$ \\
\hline Unsaturation Index * & $47.7 \pm 7.2$ & $91.6 \pm 5.8$ & $20.02(<\mathbf{0 . 0 0 0 1})$ \\
\hline n3:n6 ratio * & $2.5 \pm 0.3$ & $5.0 \pm 0.5$ & $18.96(<\mathbf{0 . 0 0 0 1})$ \\
\hline
\end{tabular}


Depth (MANOVA, p < 0.01) and block (MANOVA, p < 0.05) influenced the nutritional quality of summer biofilm (Table A5.6A). Depth did not influence the nutritional quality of fall biofilm (MANOVA, $\mathrm{p}>0.05$; Table A5.6A), however, spatial variability was also observed (MANOVA, p < 0.0001; Table A5.6A). Summer biofilm incubated at 25 and $50 \mathrm{~cm}$ from the surface had higher $\sum$ n-3 PUFA and EPA+DHA content than biofilm incubated at 125 and 150 $\mathrm{cm}$, however, no discrete depth-related pattern was observed in the $\sum \mathrm{n}-6$ PUFA content of summer biofilm (Fig. 3.14A). On the contrary, the overall yield of $\sum$ n-3 PUFA, $\sum$ n-6 PUFA, and EPA+DHA increased with depth in summer biofilm (Table 3.2). Fall biofilm incubated at greater depths had higher $\sum$ n-6 PUFA content (Fig. 3.14B). No differences were observed in $\sum$ n-3 PUFA and EPA+DHA content when pairwise comparisons were conducted on fall biofilm between all depth combinations (Fig. 3.14B). The overall yield of $\sum$ n-3 PUFA, $\sum$ n-6 PUFA, and EPA+DHA in fall biofilm also increased with depth, however unlike summer biofilm, a maximum was observed at $100 \mathrm{~cm}$, after which values decreased (Table 3.2).

Table 3.2: Average values for the overall yield $\left(\mathrm{mg} / \mathrm{m}^{2}\right)$ of nutritional fatty acids from biofilm at various depths collected in summer and fall. Values are presented as mean \pm SD. The difference between seasons was determined by a Student's t-test and a * represents significant difference between seasons. Pairwise comparisons (Tukey HSD post-hoc test) were done on each response separately after data were separated by season (to remove bimodal distribution). Groups marked with different letters are significantly different between depths within a single response variable.

\begin{tabular}{cccc}
\hline Depth $(\mathrm{cm})$ & $\sum \mathrm{n}-3$ PUFA & $\sum \mathrm{n}-6$ PUFA & EPA+DHA \\
\hline \hline Summer & $20.1 \pm 8.2^{*}$ & $8.3 \pm 3.8^{*}$ & $10.8 \pm 4.1^{*}$ \\
\hline \hline 25 & $6.6 \pm 1.6^{\mathbf{a}}$ & $2.2 \pm 0.5^{\mathbf{a}}$ & $4.1 \pm 1.0^{\mathbf{a}}$ \\
50 & $14.8 \pm 1.2^{\mathbf{a b}}$ & $5.6 \pm 0.4^{\mathbf{a b}}$ & $8.6 \pm 0.8^{\mathbf{a b}}$ \\
75 & $21.9 \pm 6.8^{\mathbf{b c}}$ & $8.6 \pm 2.9^{\mathbf{b c}}$ & $11.9 \pm 3.5^{\mathbf{b}}$ \\
100 & $25.0 \pm 2.6^{\mathbf{b c}}$ & $10.4 \pm 1.5^{\mathbf{c}}$ & $13.6 \pm 1.3^{\mathbf{b}}$ \\
125 & $25.6 \pm 3.0^{\mathbf{b c}}$ & $11.3 \pm 1.4^{\mathbf{c}}$ & $13.0 \pm 1.4^{\mathbf{b}}$ \\
150 & $26.8 \pm 5.9^{\mathbf{c}}$ & $11.6 \pm 1.9^{\mathbf{c}}$ & $13.9 \pm 3.7^{\mathbf{b}}$ \\
\hline \hline Fall & $13.1 \pm 1.7^{*}$ & $2.7 \pm 0.5^{*}$ & $7.7 \pm 0.8^{*}$ \\
\hline \hline 25 & $11.9 \pm 0.8^{\mathbf{a}}$ & $2.0 \pm 0.2^{\mathbf{a}}$ & $7.9 \pm 0.2^{\mathbf{a b}}$ \\
50 & $13.4 \pm 0.9^{\mathbf{a b}}$ & $2.6 \pm 0.2^{\mathbf{a b}}$ & $8.1 \pm 0.3^{\mathbf{a b}}$ \\
75 & $13.5 \pm 1.2^{\mathbf{a b}}$ & $2.8 \pm 0.3^{\mathbf{b c}}$ & $7.8 \pm 0.4^{\mathbf{a b}}$ \\
100 & $15.4 \pm 1.6^{\mathbf{b}}$ & $3.4 \pm 0.4^{\mathbf{c}}$ & $8.7 \pm 0.5^{\mathbf{a}}$ \\
150 & $13.1 \pm 0.4^{\mathbf{a b}}$ & $2.8 \pm 0.1^{\mathbf{b c}}$ & $7.4 \pm 0.0^{\mathbf{b c}}$ \\
\hline
\end{tabular}


Summer biofilm incubated at 25 and $50 \mathrm{~cm}$ had higher 16:1n-7 content than biofilm incubated at 100, 125, and $150 \mathrm{~cm}$ (Fig. 3.15A). The proportion of 16:1n-7 (of $\sum \mathrm{FA}$ ) was higher in shallow biofilm (i.e., 25-75 cm) compared to deeper biofilm (i.e., $150 \mathrm{~cm}$; Tukey HSD, $\mathrm{p}<$ 0.05). Pairwise comparison between the $16: 1 \mathrm{n}-7$ content and proportion of fall biofilm showed no differences between depths (Fig. 3.15A). Similarly, summer biofilm incubated at $100 \mathrm{~cm}$ had lower odd-chained FA than biofilm incubated at 25 and $50 \mathrm{~cm}$ (Fig. 3.15B). Neither depth (Fig. 3.15B) nor block had an influence on the sum of odd-chained FA in fall biofilm (ANOVA, p > 0.05; Table A5.7F).

Spatial variability was only observed in the FA biomarkers and limited to summer biofilm (Table A5.6E-F). Summer biofilm incubated in Block B $(6.1 \pm 1.1 \mu \mathrm{g} / \mathrm{mg}$ AFDW) had lower 16:1n-7 content than both Block A $(6.8 \pm 1.1 \mu \mathrm{g} / \mathrm{mg}$ AFDW $)$ and C (6.9 $\pm 1.1 \mu \mathrm{g} / \mathrm{mg}$ AFDW; Tukey HSD, $\mathrm{p}<0.05$; Table A5.6E). Similar to the diatom biomarker, summer biofilm incubated in Block B $\left(0.4 \pm 0.04 \mu \mathrm{g} / \mathrm{mg}\right.$ AFDW) had lower $\sum$ odd-chained FA than both Block A $(0.5 \pm 0.02 \mu \mathrm{g} / \mathrm{mg}$ AFDW $)$ and C $(0.5 \pm 0.03 \mu \mathrm{g} / \mathrm{mg}$ AFDW; Tukey HSD, $\mathrm{p}<0.05$; Table A5.6F).

Total FA, $\sum$ SFA, $\sum$ MUFA, $\sum$ PUFA, the percentage of SFA, MUFA, and PUFA of Total FA, as well as n3:n6 ratio were all used as summary indices to determine potential changes in the biofilm FA profile. The UI and 18:1n-9 were used to determine potential temperaturerelated differences between seasons or depths. All summary indices were influenced by season as all responses were lower in summer biofilm, except MUFA\% and PUFA\% which were higher in fall biofilm (Table 3.1).

In summer months, depth strongly influenced FA summary indices (MANOVA, $\mathrm{p}<0.01$; Table A5.8A), while block did not (MANOVA, p > 0.05; Table A5.8A). Summer biofilm incubated at $25 \mathrm{~cm}$ typically had higher $\sum$ SFA, $\sum$ MUFA, and $\sum$ PUFA content than biofilm incubated at 125 and $150 \mathrm{~cm}$, however, a similar effect was also observed in $\sum$ FA (Table 3.3). The proportion of SFA was higher in summer biofilm incubated at $125 \mathrm{~cm}$, compared to biofilm incubated at 25 and $50 \mathrm{~cm}$, however, no differences were observed in the proportion of MUFA and PUFA between depths (Table 3.3). The UI decreased with depth in summer biofilm as the UI of deeper biofilm ( $\geq 75 \mathrm{~cm}$ ) was lower than the UI of biofilm incubated at 25 and $50 \mathrm{~cm}$ (Fig. 3.16). 


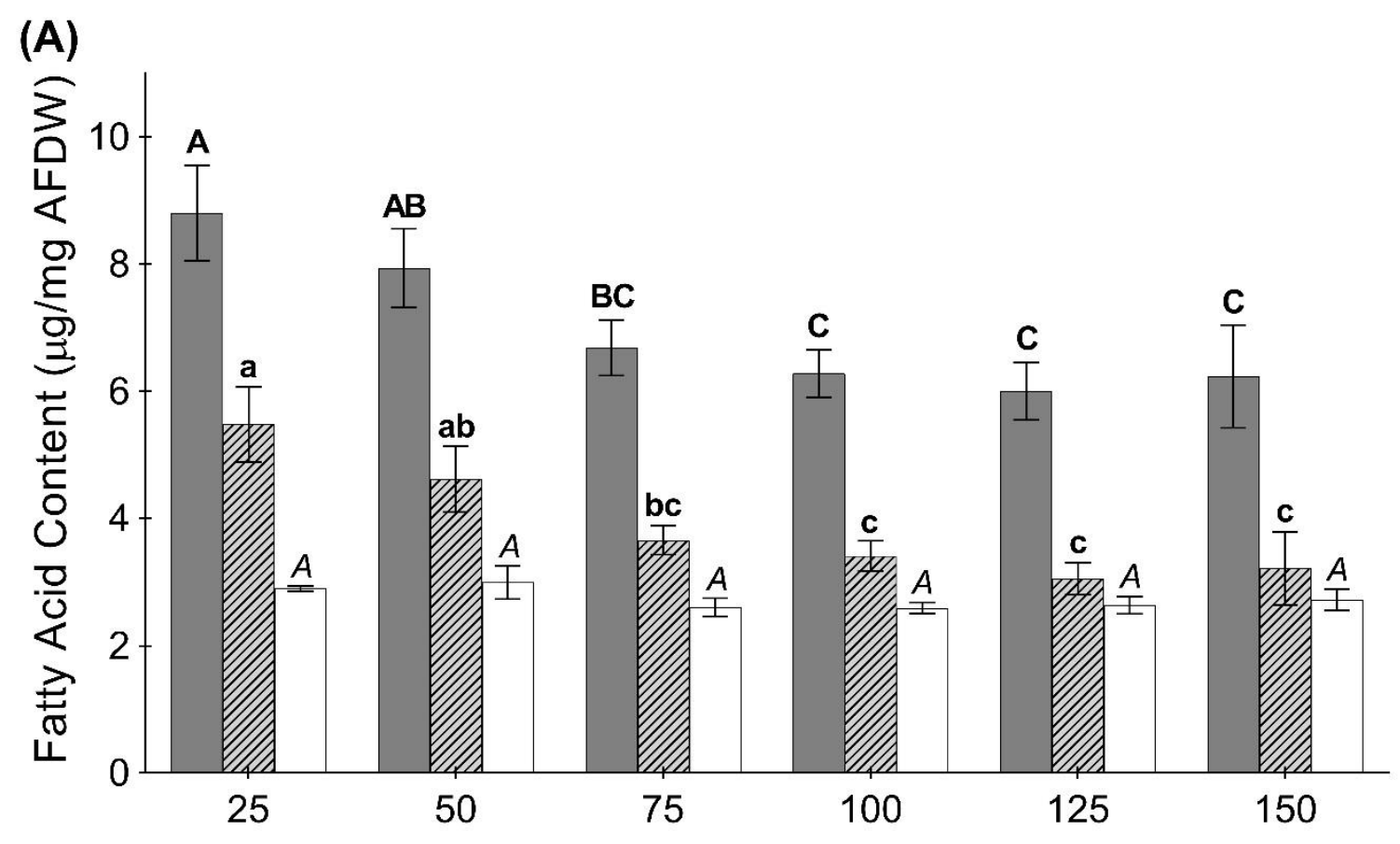

(B)

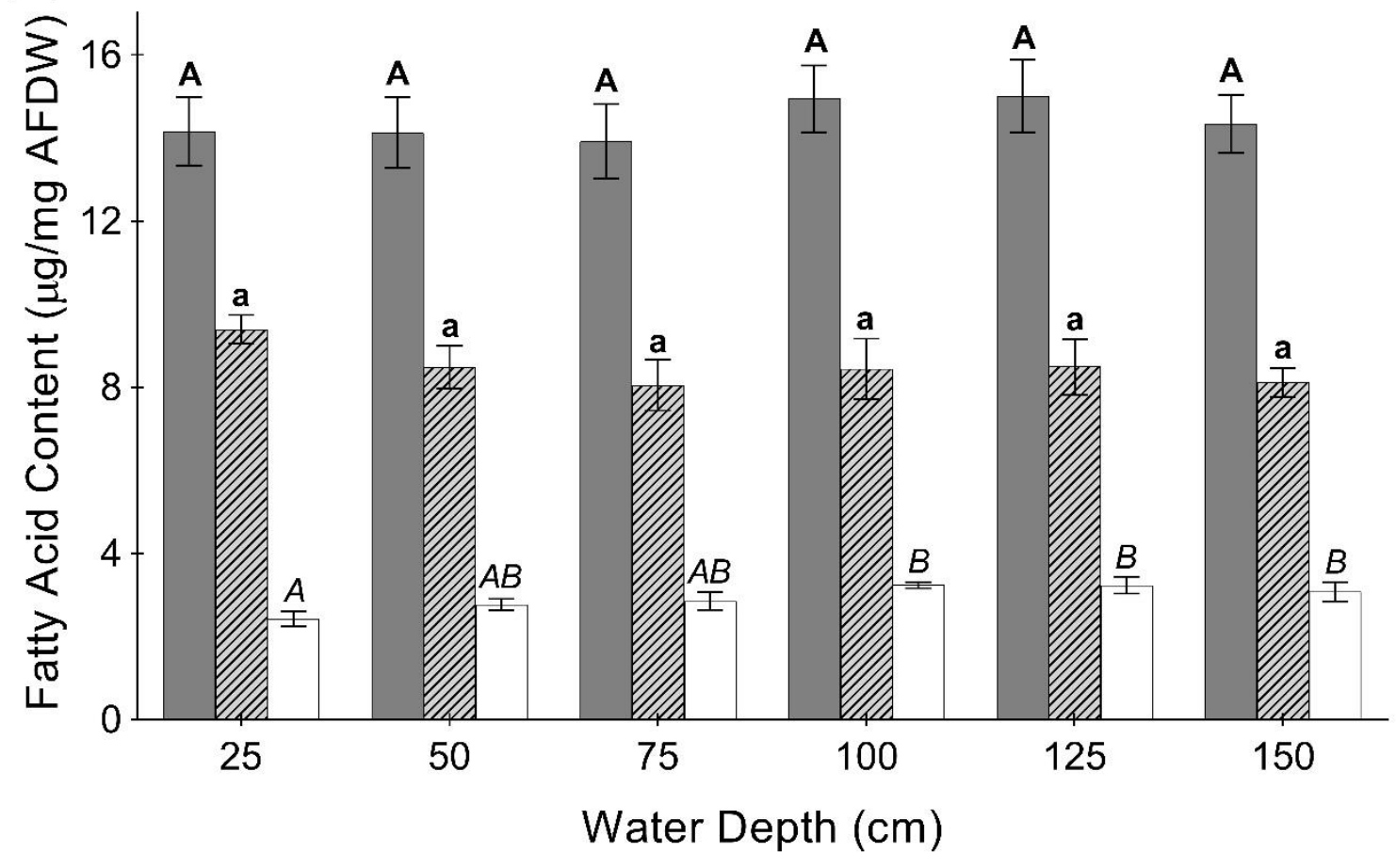

Figure 3.14: Nutritional quality of biofilm incubated at depths in (A) summer and (B) fall determined by $\sum$ n-3 PUFA (dark grey fill), EPA + DHA (stripped light grey fill), and $\sum$ n-6 PUFA (white fill) content. Depths marked with different letters represent groups that are significantly different within one response (Tukey HSD post-hoc test): $\sum$ n-3 PUFA (bolded upper case), EPA + DHA (bolded lower case), and $\sum$ n-6 PUFA (italicized upper case). 


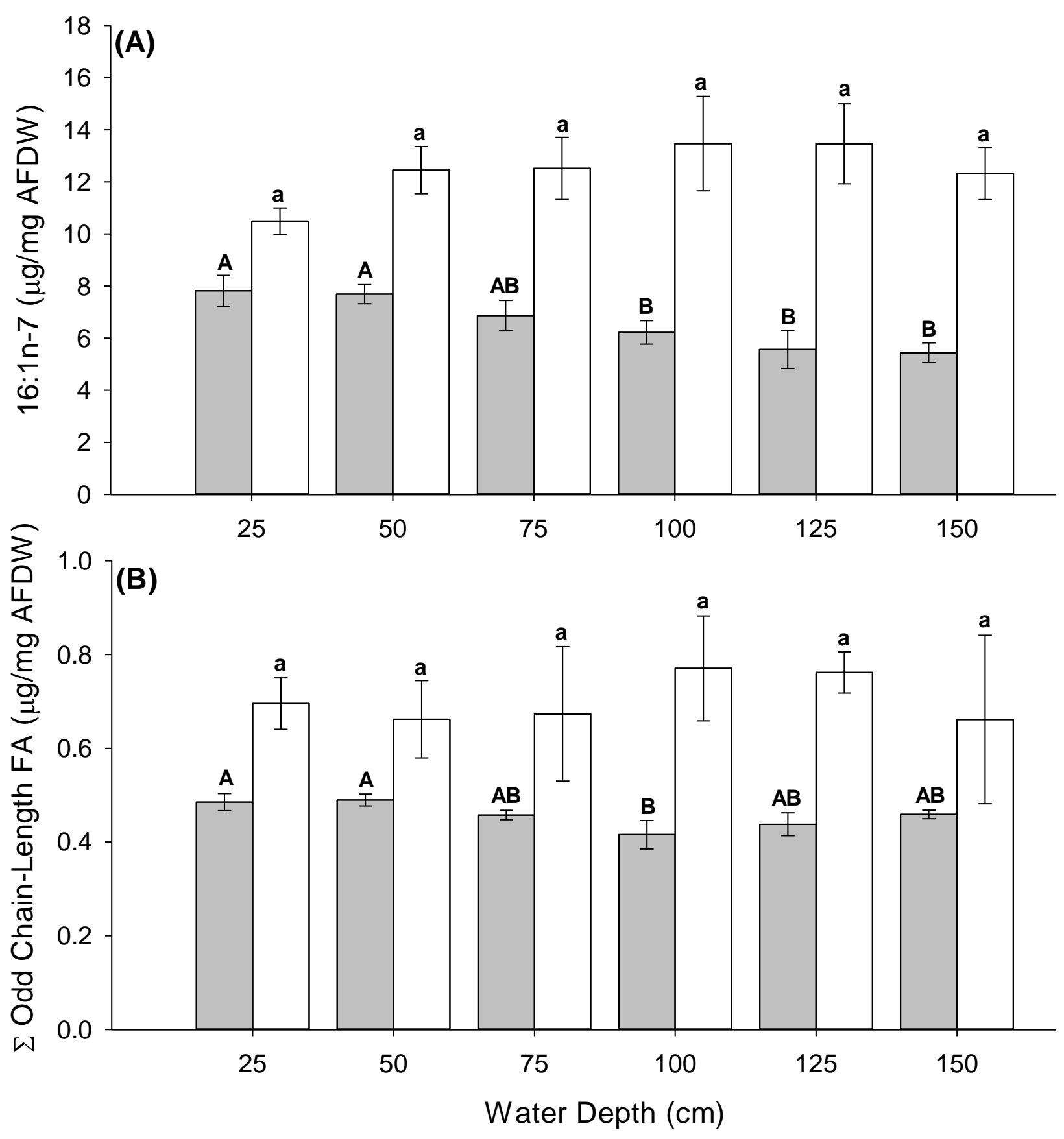

Figure 3.15: FA biomarker content in summer (grey fill) and fall (white fill) biofilm incubated at several depths. The FA (A) 16:1n-7 is a diatom biomarker and (B) $\sum$ odd chain-length FA are bacterial biomarkers. Groups marked with different letters are significantly different (Tukey HSD post-hoc test). Upper (summer) and lower (fall) cases represent separate post-hoc tests as seasons were analyzed separately. 
In fall biofilm, depth marginally influenced FA summary indices (MANOVA, $\mathrm{p}=0.068$; Table A5.9A). No depth-related effect was observed in $\sum$ FA and $\sum$ PUFA in fall biofilm, however, fall biofilm incubated at 100 and $125 \mathrm{~cm}$ had higher $\sum$ SFA and $\sum$ MUFA content than biofilm incubated at $25 \mathrm{~cm}$ (Table 3.3). Similar to summer biofilm, the proportion of SFA was higher in fall biofilm incubated at 100 and $125 \mathrm{~cm}$ compared to $25 \mathrm{~cm}$ (Table 3.3). Similarly, the proportion of MUFA was higher in fall biofilm incubated at 75 and $125 \mathrm{~cm}$ compared to $25 \mathrm{~cm}$ (Table 3.3), however, no differences were observed in the proportion of PUFA in fall biofilm between depths (Table 3.3). A similar relationship was observed in the 18:1n-9 content and n3:n6 ratio of fall biofilm (Table 3.3). No differences were observed in the UI between biofilm incubated at different depths in the fall (Fig. 3.16).

Spatial variability was observed in the summer biofilm (MANOVA, $\mathrm{p}<0.01$; Table A5.8A), however, only marginal block effects were observed in $\sum$ PUFA, UI, and 18:1n-9 (Table A5.8E-G). Spatial variability was also observed in the fall between the $\sum$ SFA content of Block A and B and the $\sum$ MUFA content of Block B and both Block A and C (Tukey HSD, p < $0.05)$.

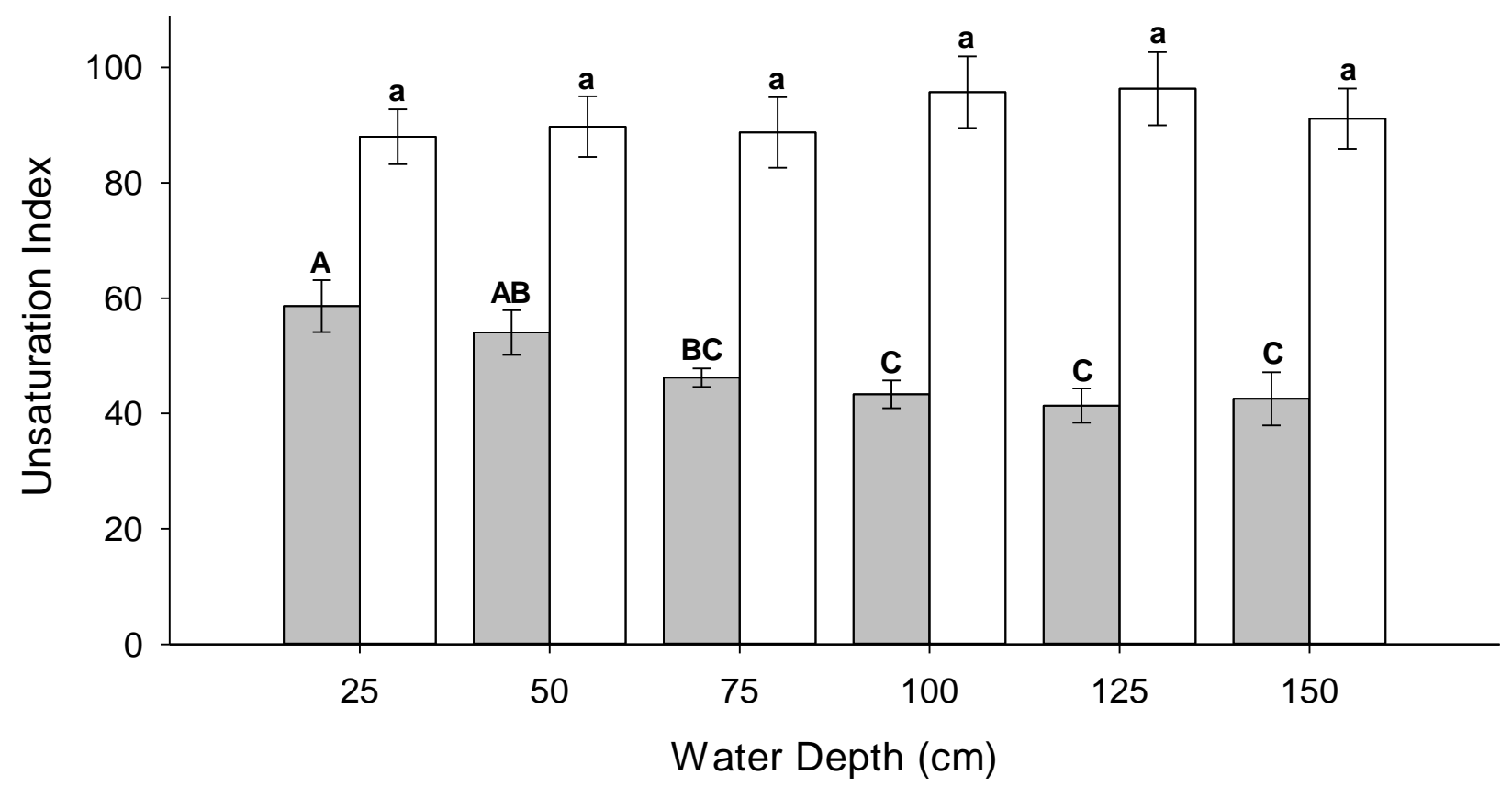

Figure 3.16: The depth-related effect on the unsaturation index (UI) of biofilm incubated in summer (grey fill) and fall (white fill). Groups marked with different letters are significantly different (Tukey HSD post-hoc test). Upper (summer) and lower (fall) cases represent separate post-hoc tests as seasons were analyzed separately. 


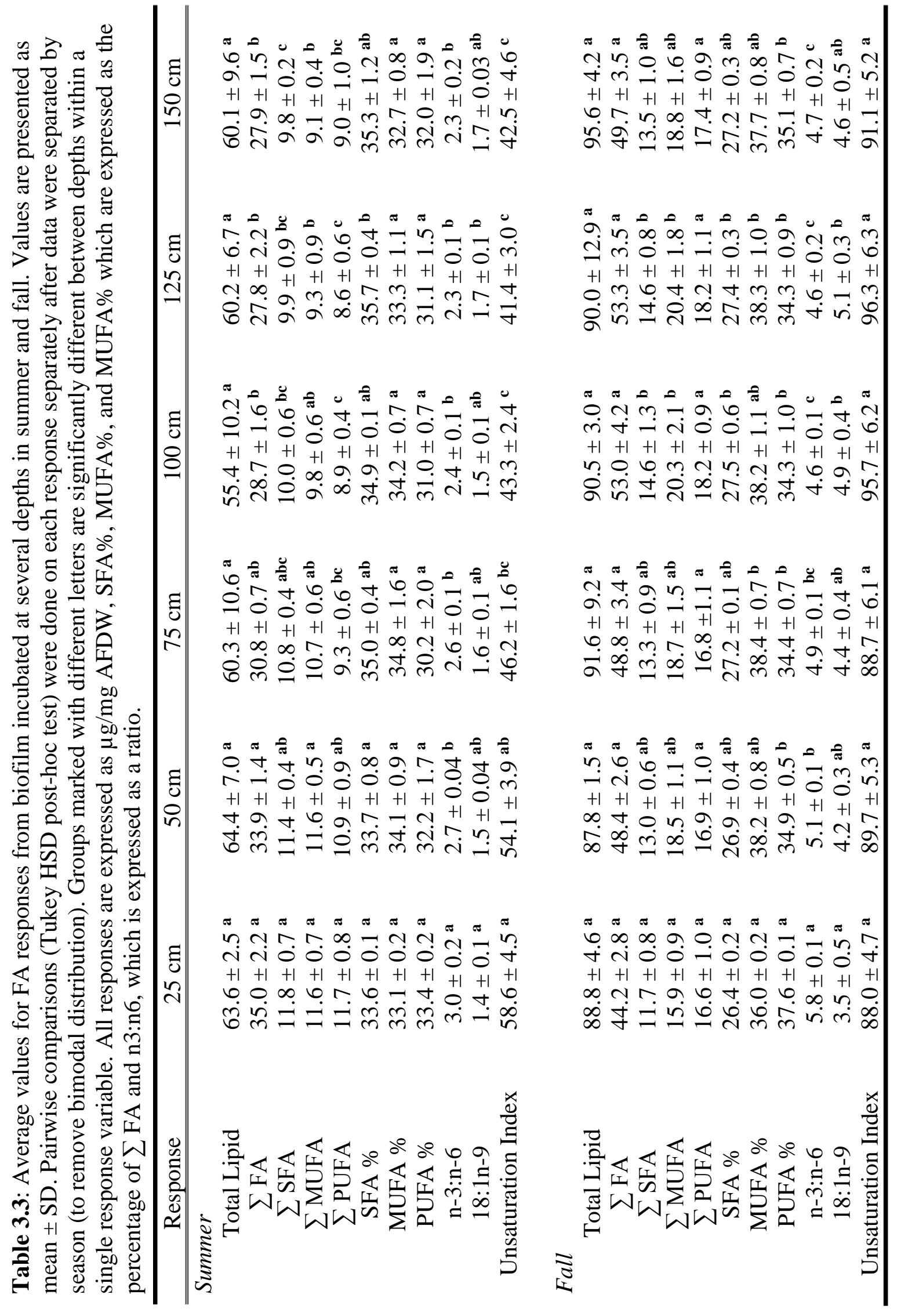




\section{$\underline{\text { 3.4 Discussion }}$}

The main objective of this study was to determine the effect of season and depth on biofilm quantity and nutritional quality. Spatial variability has been demonstrated in experiments examining freshwater biofilm (Morin \& Cattaneo 1992) and therefore, the potential influence of spatial variability was also examined. Summary indices were also examined to determine differences in the proportions of FA, which may be related to abiotic factors measured throughout the experiment. Unglazed ceramic tiles were incubated at six different depths, which were replicated across three transects (in a randomized block design) in a small freshwater pond through two seasons. Biofilm biomass was measured by DW, AFDW, and bio-volume (via CLSM) while $\sum$ n-3 PUFA, $\sum$ n-6 PUFA, and EPA+DHA content were used as an indicator for nutritional quality. Summary indices (e.g., sum and proportions of FA, SFA, MUFA, and PUFA) were used to determine any differences in the fatty acid profile, potentially related to cellular adaptation to temperature differences. The influence of seasonality, depth, and spatial variability on aquatic biofilm is likely influenced by the abiotic factors that are important to biofilm growth and development (e.g., temperature and light), which can differ between season, depths, and spatial gradient. Therefore, several abiotic factors were measured throughout the experiment, in an attempt to relate differences in the quantity and quality of field-collected biofilm to changes in abiotic factors.

\subsubsection{Abiotic Factors}

Summer temperatures were typically warmer than fall temperatures and there was a decrease in water temperature with increasing depth in summer (Fig. 3.3). Shallow ponds in the area (i.e., Whitby) have been shown to thermally stratify during summer months (Song et al. 2013), suggesting thermal stratification occurred in the study pond during the summer period. Spatial variability in water temperature was only observed in summer months and only at certain depths, however, no overall spatial trend was observed. Other abiotic factors (i.e., conductivity, TDS, pH, DO, CDOM) were affected by season but only CDOM in the summer months differed significantly between depths as higher dissolved organic matter was present at deeper depths.

The underwater light climate that biofilm was exposed to was greatly affected by both season and depth. Higher levels of PAR irradiance were available to summer biofilm as well as 
biofilm incubated at shallower depths (Fig. 3.4). Both summer and fall biofilm were exposed to similar levels of total UV radiation $(200-400 \mathrm{~nm})$, however, levels did decrease with depth (Fig. 3.5A). When separated, both UV-A (315-400 nm) and UV-B $(280-315 \mathrm{~nm})$ were higher during the summer and at shallower depths (Fig. 3.5B and C). The peak of PAR irradiance in shallow lakes has been shown to occur during late summer months (i.e., August), after which PAR decrease until peaking the following summer (Xu et al. 2010). Although not specifically measured, fall biofilm were exposed to less direct sunlight due to the reduced daylight hours and lower sun angle observed during fall months in North America. The underwater light spectra is expected to decrease with depth as light is scattered/absorbed by water, dissolved organic matter, and pelagic phytoplankton (Gallegos \& Moore 2000). The summer increase in CDOM with depth may account for some of the decreased light availability at greater depths in summer months. Based on the parameters measured, it is evident that biofilm incubated during different seasons and depths were exposed to different abiotic environments, which may have influenced their quantity and nutritional quality.

\subsubsection{Seasonal, Depth-related, and Spatial Differences in Biofilm Biomass}

The combination of both semi in-situ (i.e., CLSM analysis) and ex-situ (i.e., DW and AFDW from lyophilized samples) methods to determine biofilm structure and biomass has been used separately in laboratory settings (e.g., Arnon et al. 2007), however, their combined usage has been limited, especially with respect to environmental samples (e.g., Albers 2010). Interestingly, seasonality was not observed in bio-volume measurements in my study, however, DW and AFDW demonstrated a five- and three-fold decrease in fall biofilm biomass compared to summer biofilm biomass, respectively. Although the proportion of organic matter in fall biofilm was higher than summer, the AFDW (calculated as the DW multiplied by proportion of organic lost during ashing) clearly demonstrates that tiles incubated in the summer accumulated more organic biomass than fall biofilm. It should be noted that the accumulation of organic matter measured in this experiment may not represent true productivity of the biofilm because factors such as organic deposition, predation, and sloughing can influence the organic matter observed in biofilm. Although AFDW does not distinguish between living and non-living organic matter, higher AFDW in summer biofilm incubated on artificial surfaces has been demonstrated in both freshwater streams (Rosemond 1994) and shallow lakes (van Dijk 1993), 
confirming the typical seasonal pattern of maximal biofilm biomass during spring and summer months in freshwater ecosystems.

The lack of seasonality in bio-volume measurements may be partially due to the combination of high spatial variability of a biofilm surface that leads to high variability in in-situ measurements using CLSM (Neu \& Lawrence 2016). The proportion of DW and moisture content of biofilm may have influenced bio-volume measurements as summer biofilm had lower moisture content but higher DW and AFDW and vice-versa for fall biofilm. Additionally, due to the time and logistical constraints, only a single tile was used to represent each incubator, instead of three tubes containing triplicate tiles as with DW and AFDW measurements. CLSM also revealed that fall biofilm was also, on average, thicker than summer biofilm although the spatial heterogeneity (i.e., roughness coefficient) was similar between both seasons. Biofilm EPS is composed of up to $99 \%$ water (Cooksey 1992), which may explain the higher moisture content observed in fall biofilm as CLSM was done on fully-hydrated biofilm that were placed and stored in filtered pond water prior to analysis. CLSM has been used to demonstrate maximum bio-volume in spring biofilm compared to fall biofilm grown on artificial surfaces in a wastewater treatment facility due to high diatom abundance (Congestri et al. 2006). Overall, the combination of in-situ and ex-situ measurements demonstrated that tiles incubated during summer months accrued more organic material. However, the biofilm growing on the tiles were similar in volume in summer and fall perhaps as a result of a higher moisture content in fall biofilm.

Tiles incubated at greater depths accumulated higher DW (Fig. 3.9) and AFDW (Fig. 3.11), however, this depth-related effect was only observed in summer biofilm. Deeper summer biofilm ( $\geq 75 \mathrm{~cm}$ ) had lower organic percentage (Fig. 3.12) but higher DW (Fig. 3.9) and AFDW (Fig. 3.11) than shallower biofilm $(25$ and $50 \mathrm{~cm}$ ). Greater algal biomass is typically observed near the water surface during summer months (Liboriussen \& Jeppesen 2009; Kreuzinger-Janik et al. 2015), where the high levels of PAR irradiance can stimulate algal growth. Chlorophyll samples were not analyzed for this experiment, however, higher UV (-R, -A, and -B) observed at shallower depths during both seasons may have also reduced biofilm biomass near the water surface, as UV radiation can reduce AFDW and densities of cellular biovolume (Vinebrooke \& Leavitt 1996). High levels of AFDW on deeper tiles in summer months may also be due to high levels of detritus (i.e., non-living organic matter; Wilson et al. 2003) caused by phytoplankton 
deposition or settling after pelagic phytoplankton blooms in the summer (Goedkoop \& Johnson 1996). Phytoplankton deposition would also provide nutrients (i.e., phosphorus and nitrogen) to microorganisms within deeper biofilm, potentially stimulating biofilm growth. In a small pond, deeper biofilm may also benefit from nutrients released from the sediment during the summer stratified months as shallow ponds in Whitby have been shown to have higher phosphorus accumulation during summer months (Song et al. 2013). This may result in higher living organic matter on deeper tiles in the summer, however, fall turnover would distribute nutrients more or less equally within the water column. Unfortunately, dissolved nutrients were not analyzed during this study. Finally, although the direct effect of grazing pressure or sloughing was not measured, a decrease in the spatial heterogeneity (i.e., roughness coefficient) of biofilm with depth suggests higher grazing pressure near the surface. Biofilm grazers create a more heterogenous biofilm structure while in the absence of predators (grazers), biofilm develop a homogenous, compact, and thick structure (Derlon et al. 2012, 2013). Visual inspections of tiles prior to harvesting also suggested shallower tiles were heavily grazed, relative to deeper tiles, due to presence of blank substrate surface marked by distinct grazing tracks.

The seasonality of ex-situ measurements has been demonstrated, however, shallow biofilm in spring/summer typically have higher DW (Eloranta 1982). Liboriussen and Jeppesen (2009) found no systematic difference in biofilm DW between biofilm obtained from 5, 40, and $75 \mathrm{~cm}$ during winter but did find more biomass near the surface during the spring in a shallow lake. The relationship between depth and biofilm may vary depending on the characteristic of the system as biofilm collected from a clear macrophyte-dominated eutrophic lake had a decrease in dry weight from 20 to $40 \mathrm{~cm}$ while an increase in dry weight with depth was observed in phytoplankton-turbid lake (Sánchez et al. 2013). Although my study pond was characterized as mesotrophic (based on nutrient concentrations in Appendix 3), high abundance of phytoplankton during the summer would characterize it as phytoplankton-turbid (Scheffer et al. 1993), suggesting a positive relationship between depth and biofilm biomass in productive waterbodies. No clear depth pattern was observed in the DW, AFDW, organic percentage, or moisture content between depths of fall biofilm, however, CLSM were able to reveal differences at a finer scale.

Regardless of season, biofilm incubated at greater depths had higher bio-volume (Fig. 3.6) and mean thickness (Fig. 3.7). Although no depth-related pattern was observed in fall biofilm using ex-situ methods, an increase in bio-volume (Fig. 3.6) and mean thickness (Fig. 3.7) 
was evident using CLSM. The difference between in-situ and ex-situ measurements may be related to the resolution of each technique. All DW and AFDW were measured using an analytical balance with a resolution of $\pm 1 \mathrm{mg}$, while CLSM measurements were conducted on a resolution scale $10^{4}$ smaller (i.e., $\pm 0.01 \mu \mathrm{m}$ ), therefore, depth-related differences seen the biovolume and mean thickness of fall biofilm may have been undetectable in larger-scaled measurements like DW and AFDW. The advantages of using CLSM are in its ability to examine aquatic biofilm within its environmental context (i.e., hydrated and attached to a substrate) and detect fine-scale in-situ structural characteristics of biofilm that are otherwise lost when the biofilm is scraped off the substrate (Neu \& Lawrence 2016). Therefore, using traditional ex-situ methods, a depth-effect was only observed in the summer while using in-situ revealed a finerscaled increase in biofilm biomass with increasing depth in both seasons.

Spatial variability was observed in both biomass measurements but were mostly limited to summer biofilm. Summer biofilm incubated in Block $\mathrm{C}$ had lower bio-volume (Fig. 3.7) and organic matter percentage (Fig. 3.13) but higher heterogeneity and DW (Fig. 3.10) than both Block A and B. Although spatial variability was observed in most biomass parameters, there were no differences observed in the organic matter (i.e., AFDW) on tiles incubated in summer and fall between the blocks. Spatial variation in biofilm AFDW has been observed in a largescale (i.e., 6,000 $\mathrm{km}^{2}$ ) gradient and in streams with differing abiotic factors, such as light (Rosemond 1993; Rosemond et al. 2000; Godwin \& Carrick 2008). Differences in spatial variability in dry weights and bio-volume may be explained by higher exposure to UV. Although, not measured due to logistic difficulties taking light measurements at different locations, Block C represents a more "open-water" area and compared to Block A and B (Fig. 3.1). Overall, seasonal, depth-related, and spatial variability was observed in the quantity of biofilm, as higher biomass is typically observed at greater depths, however, interestingly, depth has the opposite effect on the nutritional quality (discussed below).

\subsubsection{Seasonal and Depth-related Differences in Biofilm Nutritional Quality}

Most parameters in the lipid data, when both seasons were included, had a multimodal distribution (Hartigans' dip test, $\mathrm{p}<0.05$; Table 3.1), however, when data were separated by season, all response variables in both seasons were unimodal and normally-distributed (Hartigans' dip test; $\mathrm{p}>0.05$ ). The nutritional quality of biofilm was determined by the $\sum \mathrm{n}-3$ 
PUFA, $\sum$ n-6 PUFA, and EPA+DHA content. All three indicators of nutritional quality were higher in fall biofilm than summer biofilm (Table 3.1). This would suggest that in terms of physiologically-important FA, fall biofilm (standardized for AFDW) contain higher content compared to summer biofilm, which may be related to the temperature-mediated increase in PUFA proportions observed at colder temperatures (discussed in next section). Although fall biofilm contain higher PUFA content per standardized unit of mass, interestingly, the overall yield of PUFA was higher in the summer (Table 3.2). This suggests high availability of PUFA in the summer, however, a larger amount of biofilm would need to be consumed by a grazer to ingest the same amount as available in fall biofilm.

Along with the nutritional indicators used, $\sum$ FA was lower in summer than fall (Table 3.1). Sushchik et al. (2010) and Sanpera-Calbet et al. (2017) also reported lower $\sum$ FA in the summer, however, peak values were observed in spring biofilm, which were not collected in this study. Higher lipid content in fall biofilm may provide biofilm microorganisms sufficient energy storage during low-light and low-nutrient winter months and ultimately influence biofilm growth in the following spring season. Studies have demonstrated the seasonality of biofilm FA, most of which reported individual values as a proportions of total FA (e.g., Torres-Ruiz et al. 2007; Sushchik et al. 2010) or of total chromatographed peak area (e.g., Honeyfield \& Maloney 2015). Although FA proportions are essential in examining trends in the overall FA profile (discussed in next section), the nutritional quality of biofilm can more reasonably be quantified by examining the content $(\mu \mathrm{g})$ standardized to organic mass (i.e., on a per mg AFDW basis). The presence of EPA in the biofilm FA profile and a DHA/EPA ratio < 1 (values ranged from 0.10 to 0.48 ) also strongly suggest the dominance of diatoms (Budge \& Parrish 1998; Dalsgaard et al. 2003). The increase in nutritional quality in the fall was also accompanied by an increase in palmitoleic acid (16:1n-7), a common biomarker for diatoms (Taipale et al. 2013). This may be due to increased abundance of pelagic diatoms in the fall due to cooler temperatures and silica recovery (Hecky et al. 1986; Munawar \& Munawar 1986), which may increase diatom abundance within the biofilm by increasing the deposition of living or dead diatoms, along with their PUFA content. This seasonal variation in biofilm FA is ecologically important because FA are involved in many cellular functions (Ibarguren et al. 2014; Glatz \& Luiken 2015; Schönfeld \& Wojtczak 2016) and therefore, grazers may have limited access to physiologically important FA throughout the year. 
Within-season depth-related variation was also observed in this study as shallow biofilm (i.e., 25 and $50 \mathrm{~cm}$ ) in the summer had higher $\sum \mathrm{n}-3$ PUFA and EPA+DHA content than deeper biofilm (i.e., 125 and $150 \mathrm{~cm}$; Fig 3.14A). Interestingly, the overall yield of $\sum \mathrm{n}-3$ PUFA, $\sum \mathrm{n}-6$ PUFA, and EPA+DHA were also higher at summer biofilm incubated at greater depths (Table 3.2). This suggests that although shallower summer biofilm contain higher standardized PUFA content, greater PUFA quantity is available at greater depths. Therefore, grazers feeding on deeper biofilm would need to ingest more biofilm to receive the same mass of PUFA available in shallower biofilm. Aquatic snails, common biofilm grazer, are commonly observed at deeper depths in the littoral zone of lakes (James et al. 1998; Weatherhead \& James 2001; Schroeder et al. 2013). Although their vertical distribution may be related to their preference for sheltered surfaces (DeNicola \& McIntire 1991), aquatic snails are efficient grazers of biofilm (Peters et al. 2012) as they can remove biofilm $>100 \mu \mathrm{m}$ thick (Lawrence et al. 2002) and even crustose algae (Neumann 1961) and therefore, may benefit from the higher PUFA availability at greater depths. In the fall, deeper biofilm had higher n-6 PUFA content, however, no differences were observed in $\sum$ n-3 PUFA and EPA+DHA content between depths (Fig. 3.14B). Interestingly, the maximum yield of PUFA was observed at $100 \mathrm{~cm}$ (Table 3.2). This might suggest an optimal depth, due to a combination of abiotic and biotic factors in the fall season, for biofilm quantity and PUFA content.

Increasing depth has been shown to increase the atomic ratio of $\mathrm{C}: \mathrm{N}$ and $\mathrm{C}: \mathrm{P}$ of epilithic biofilm, which suggests a reduction in elemental nutritional quality (Kahlert et al. 2002). This study demonstrated that nutritional quality of aquatic biofilm, in terms of physiologicallyimportant n-3 PUFA, also decrease with depth, although this effect was limited to the summer period. The high abundance of palmitoleic acid (16:1n-7) in biofilm collected from this study (values ranged from 36.7 to $53.6 \%$ ) suggests the dominance of diatoms, which likely contribute to the nutritional quality of biofilm due to their ability to produce high quantities of PUFA, especially n-3 varieties (Lang et al. 2011). In summer biofilm, the $\sum n-3$ PUFA and EPA+DHA content decreased with depth (Fig. 3.14A), as well as the palmitoleic acid content (Fig. 3.15A). The proportion of palmitoleic acid (of total FA) also significantly decreased with depth from $22.3 \pm 0.3 \mu \mathrm{g} / \mathrm{mg}$ AFDW at 25 from the surface to $19.5 \pm 0.8 \mu \mathrm{g} / \mathrm{mg}$ AFDW at $150 \mathrm{~cm}$ from the surface. The content and proportion of palmitoleic acid both suggest that diatom abundance in summer biofilm decreased with depth, which would provide an explanation for the reduction in 
PUFA. This is consistent with other studies that have reported high diatom abundance in shallow biofilm (i.e., $60 \mathrm{~cm}$ from the surface), where sufficient levels of PAR are available for optimal growth (Yang \& Flower 2012). No depth-related differences were found in the $\sum$ n-3 PUFA content of fall biofilm between depths, as well as in the content and proportion of palmitoleic acid (Fig. 3.15A). Overall, the PUFA content of biofilm differs seasonally and between depths, albeit limited to summer months; therefore, the availability of physiologically-important FA can potentially differ for grazers between seasons and along a depth gradient.

\subsubsection{Seasonal and Depth-related Differences in Biofilm Fatty Acid Profile}

The PUFA content was used to determine nutritional value, however, the content (i.e., $\mu \mathrm{g} / \mathrm{mg}$ AFDW) and proportion (\% of total FA measured) of SFA, MUFA, and PUFA were used as indicators of the overall fatty acid profile of aquatic biofilm. The $\sum \mathrm{FA}$, as well as the $\sum$ SFA, $\sum$ MUFA, and $\sum$ PUFA, increased in fall biofilm compared to summer biofilm (Table 3.1). However, the proportions of SFA decreased in fall biofilm while the proportions of MUFA and PUFA increased in fall biofilm (Table 3.1). This seasonal shift in FA proportions has been observed in several studies examining biofilm FA (Sushchik et al. 2010; Guo et al. 2015; Honeyfield \& Maloney 2015). Sushchik et al. (2010) suggested a change in species composition can partially explain the differences in proportion, however, diatoms and green algae grown in warmer temperatures during summer months in that study had lower PUFA proportions suggesting temperature adaptation was occurring between seasons. Similarly, pelagic diatom population in the spring were rich in PUFA while the population in the summer with the same taxa had lower PUFA content (Sushchik et al. 2004). The UI also increased in fall biofilm (Table 3.1) in my study, potentially indicating the switch of SFA-dominated membranes to PUFAdominated membranes as cellular adaptation mechanism to cooler temperatures. The content of a MUFA, specifically 18:1n-9, also increased in the fall biofilm (Table 3.1), which has been suggested as an important MUFA for adaptation to cooler temperatures (Bly et al. 1990; Teoh et al. 2004, 2013). Although not experimentally-manipulated, temperature may potentially explain seasonal differences in the proportion of SFA and PUFA in biofilm and has been suggested by other authors who have similarly observed higher PUFA proportions in fall/winter biofilm (e.g., Guo et al. 2015; Honeyfield \& Maloney 2015). 
Temperature differences were also observed between depths, specifically in summer months when deeper biofilm were exposed to cooler temperatures (Fig. 3.3). Deeper biofilm in the summer had higher $\sum$ FA, $\sum$ SFA, $\sum$ MUFA, and $\sum$ PUFA content, however, only the proportion of SFA significantly differed between depths (Table 3.3). Although a statistically significant temperature difference was observed between all depths in the summer (Fig. 3.3), the difference between the mean temperatures at 25 and $150 \mathrm{~cm}$ was $\sim 0.8^{\circ} \mathrm{C}$. Laboratory experiments that attempt to induce a biochemical response in phytoplankton FA proportions typically use a temperature difference $\geq 4^{\circ} \mathrm{C}$ (Sushchik et al. 2003b; Fuschino et al. 2011) as differences in PUFA proportions are only observed when temperature differences are $\geq 3^{\circ} \mathrm{C}$ (Renaud et al. 2002; Teoh et al. 2004). Therefore, the small difference observed in temperatures between depths may not affect the membrane fluidity of microorganisms and no temperature adaptation response would be observed. Overall, seasonality strongly influences proportions of FA, likely related to differences in temperature, however, within-season depth-related differences in temperature did not cause a shift in FA proportions.

\subsubsection{Conclusion}

The main objective of this study was to determine the effect of season and depth on the quantity and nutritional quality of aquatic biofilm. The depths were replicated in three transects to account and test for spatial variability across the pond. Traditional ex-situ measurements (i.e., DW and AFDW) demonstrated a clear decrease in biofilm biomass in the fall, as well as a summer-limited increase in biomass with increasing depth. Seasonality was not observed in CLSM indicators of biomass, however, an increase in biofilm was observed in both seasons suggesting that the usage of in-situ microscopy of aquatic biofilm may reveal finer-scaled characteristics that are undetectable in traditional measurements of biomass. Although the usage of CLSM has increased with field-incubated samples (e.g., Proia et al. 2012), adequate sample preservation and the lack of laser penetration into thick $(>150 \mu \mathrm{m})$ biofilm prevent the widespread usage of CLSM for environmental samples (Neu \& Lawrence 2016). Spatial variability was typically observed in summer biomass measurements, demonstrating the seasonality of biofilm spatial heterogeneity within a small pond ecosystem. Despite the logistical challenges associated with CLSM, its usage in combination with traditional methods can 
determine both large and smaller-scaled changes in biofilm biomass, especially in response to important abiotic factors.

Nutritional quality of biofilm was determined by the PUFA content, which increased generally in biofilm incubated in the fall but decreased with depth only in summer biofilm. The total availability of PUFA, however, was higher in the summer and at deeper depths due to the greater accumulation of organic matter. The seasonal and depth-related trends in standardized PUFA content corresponds with the content of palmitoleic acid, a common biomarker for diatoms, and which are typically also PUFA-rich algae. Therefore, the diatom abundance between seasons and within depths likely plays a key role in determining the availability of physiologically-important PUFA. Season also had an influence on the overall profile of aquatic biofilm as proportions of MUFA and PUFA increased in the fall, at the expense of SFA. This phenomenon been observed in other biofilm studies (e.g., Honeyfield \& Maloney 2015) and is potentially caused by seasonal changes in species composition and cellular adaptation to colder temperatures by microorganisms within the biofilm. The increase in palmitoleic acid in fall suggests an increase of diatom abundance in fall biofilm, however, an increase in the UI and oleic acid in the fall also suggests temperature adaptation of biofilm microbes to colder temperatures. Although biofilm incubated at different depths in the summer experienced different temperature regimes, the shift in FA proportions was not evident suggesting that depthrelated differences in temperature may not be substantial enough to induce temperature adaptation in biofilm microbes. The depth-related variation in biofilm FA has important implications for the access of physiologically important PUFA for biofilm grazers, especially those that have depth-related distribution patterns.

The basic understanding of the importance of biofilm as a source of physiologicallyimportant FA to grazers and the factors that influence their nutritional quality is limited. This study supports seasonal differences in PUFA reported in previous studies but also demonstrates the decrease of biofilm PUFA content (standardized for AFDW) with increasing depth. Common biofilm grazers, such as insect larvae (Thorp \& Diggins 1982; Wissinger 1988), nematodes, and oligochaetes (Schroeder et al. 2013; Kreuzinger-Janik et al. 2015) have been shown to have higher abundance at shallower depths in the littoral zone of lakes. The biomass and PUFA content of emerging amphibiotic insects has also been shown to decrease in deeper waters (Martin-Creuzburg et al. 2017). Although other factors may influence the depth distribution of 
biofilm grazers, the higher nutritional quality (per mg AFDW) observed in shallow biofilm may explain the spatial abundance patterns of some biofilm grazers. The differences in biofilm PUFA content between depths may have cross-ecosystem implications as amphibiotic insects (e.g., chironomids) that feed on aquatic biofilm have been shown to transfer substantial amounts of PUFA to terrestrial ecosystems (Martin-Creuzburg et al. 2017).

Grazing abundance and pressure was not captured in this study, however, grazing is an important top-down biotic factor that can control biofilm quantity and quality. The presence of grazers, such as snails (Hunter 1980; Jacoby 1985; Tuchman \& Stevenson 1991; Rosemond et al. 1993) and the larvae of caddisflies (Lamberti \& Resh 1983; Lamberti et al. 1987), mayflies (Colletti et al. 1987; Hill \& Knight 1987; Scrimgeour et al. 1991), and chironomids (Mason \& Bryant 1975; Eichenberger \& Schlatter 1978) has been shown to decrease the overall, and specifically, the algal biomass within freshwater biofilm. Grazing can also decrease the absolute nutrient amount per unit area of biofilm (Jacoby 1985; Mazumder et al. 1989) as algal biomass is typically reduced. In response to grazing, however, the phosphorus and nitrogen content percentage typically increase (Hunter 1980; Mulholland et al. 1991; Rosemond 1993; Rosemond et al. 1993), which may be related to the ability of grazers to also remove low-nutrient senescent cells from a biofilm. The selectivity of grazers may also alter the community composition of microbes within a biofilm (Steinman 1996), which may potentially alter the FA, specifically, the PUFA content of biofilm left behind.

Previous studies measuring biofilm quantity and FA content, like this study, typically collect biofilm remaining on a surface after an incubation period, however, biofilms can also be thought of as "cell factories" constantly releasing cells into the environment (Berlanga \& Guerrero 2016). The overall biomass and FA content that is retained and released by biofilms would represent the true yield, a productivity or rate measurement rather than a static (i.e., standing stock) measurement and would help us to more fully understand the importance biofilms as an energy and nutrient source in aquatic food webs. Future studies should also specifically examine the effect of grazing (i.e., unprotected versus grazer-protected tiles), in combination with season and depth, to determine the distribution of PUFA within aquatic biofilms. A future study manipulating both depth and grazing and examining FA profiles will contribute to a more comprehensive understanding of the availability of physiologicallyimportant FA in aquatic ecosystems. 


\section{Chapter 4}

\section{Summary and Research Recommendations}

\subsection{Summary}

In recent decades, the scientific community has come to a consensus on the reality of anthropogenic climate change (Cook et al. 2013, 2016) and have, increasingly, focused on its potential ecological effects (Parmesan \& Yohe 2003). Freshwater ecosystems support $\sim 6 \%$ of all described species, although only covering $0.8 \%$ of the Earth's surface (Dudgeon et al. 2006), and are predominately small $\left(<1 \mathrm{~km}^{2}\right)$ waterbodies (Downing et al. 2006) that are strongly influenced by warming air temperature (Gerten \& Adrian 2001; Morrill et al. 2005). Accelerated warming has already been observed in shallow waters during summer months (Coats et al. 2006; Hampton et al. 2008) and current projections estimate the GMST to be up to $4.8^{\circ} \mathrm{C}$ warmer by the end of the decade (Collins et al. 2013). Warmer water temperatures are predicted to induce homeoviscous adaptation, a biochemical response to temperature, which, if realized, would reduce the proportion of PUFA in phytoplankton worldwide (Hixson \& Arts 2016). This is significant because phytoplankton constitute a substantial portion of the autotrophic base of aquatic food webs and are the major producers of physiologically-important n-3 LC-PUFA (Hixson et al. 2015) for higher consumers.

Certain PUFA, especially EPA and DHA, are considered essential fatty acids (i.e., EFA) because higher trophic level organisms cannot synthesize them efficiently. They are, however, necessary for overall health of both vertebrates and invertebrates and must therefore be obtained from the diet. Limited work has been done to determine the effects of warming temperatures on multiple trophic levels, especially within the context of PUFA in a natural system. Therefore, the first objective of this thesis was to attempt to measure the effects of warming water temperatures on the production of EFA at the base of aquatic food webs and then to assess their transfer through the food web, as well as their re-distribution from aquatic to terrestrial ecosystems.

Laboratory studies have demonstrated a clear reduction of PUFA proportions at higher temperatures (e.g., Renaud et al. 2002; Teoh et al. 2004; Fuschino et al. 2011). However, this physiological response has not been examined in a natural system as in general, temperature manipulation in large-scale (>10 4 L) mesocosms have not been conducted (Stewart et al. 2013). 
Chapter 2 describes the methodology, parameters measured, and the efficiency of the heating system that was achieved in a large-scale in-situ temperature manipulation experiment. Briefly, I used custom-built pond curtains to divde a small pond into two large sections $(\sim 300,000 \mathrm{~L})$ that acted as an experimental and control mesocosms. A piping system, consiting of plastic and aluiumum pipes, was deployed into the experimental section and was connected to a pool heater and pump to warm the experimental mesocosm to $4^{\circ} \mathrm{C}$ above the control mesocosm. I planned to collected organisms from several key taxa and from various trophic positions during the experiment to measure the effect of warming temeprature on PUFA production (i.e., phytoplankton and biofilm), transfer (i.e., zooplankton, insect larvae, and minnows), and redistribution (i.e., adult insects). I also planned to determine the effect of warming temperatures on ecosystem-wide mercury dynamics and Daphnia metabolomics. Hourly temperature measurements concluded that warming was accomplished near the bottom of the pond (i.e., 0.5 $\mathrm{m}$ from the sediment), however, no differences in temperature were observed throughout the water column. Results and conclusions obtained from this study can, and should, be used for future experimental designs that aim to simulate warmer water temperatures in a natural system.

Water temperature and underwater light climate are two important abiotic factors that influence the quantity and nutritional quality of aquatic biofilm (see Chapter 1). Aquatic biofilm growing in different seasons and along a depth gradient will be exposed to different abiotic environments, which may ultimately alter their biomass and nutritional quality (i.e., PUFA content). Isotope studies have demonstrated the importance of biofilm as a basal resource of energy to higher consumers (Vander Zanden \& Vadeboncoeur 2002; Lau et al. 2009). In addition, PUFA, especially EPA which is found in high proportions within diatom-dominated biofilms, has been suggested to have physiological importance in insect larvae (Torres-Ruiz et al. 2010). The larvae abundance (Schroeder et al. 2013) and emergence (Martin-Creuzburg et al. 2017) of amphibiotic insects, such as chironomids, vary along a depth gradient. The magnitude (e.g., Martin-Creuzburg et al. 2017) and biological importance (e.g., Twining et al. 2016) of the PUFA export from aquatic to surrounding terrestrial environment via amphibiotic insects has recently been established. Therefore, the quantity and nutritional quality of biofilm between depths may explain grazer abundance and determine the availability of physiologically-important fatty acids in both aquatic and terrestrial ecosystems. The second objective of this thesis was 
therefore to addresses the effect of season and water depth, and associated abiotic factors, on the quantity and nutritional quality of aquatic biofilm.

The effect of season and depth on biofilm quantity has been examined in several systems, however, their influence on biofilm PUFA content has not been explored thoroughly. Chapter 3 describes the relationship between season depth and biofilm biomass, as well FA profile and PUFA content. Briefly, tiles were incubated at six different depths (i.e., 25, 50, 75, 100, 125, and $150 \mathrm{~cm}$ from the surface), replicated in three transects across a small pond during both summer and fall months. Both traditional ex-situ (i.e., DW and AFDW) and in-situ (i.e., CLSM) methods were used to measure biofilm biomass. Differences in the results obtained from both methods highlight the advantages of each technique and importance of using both to measure the influence of important abiotic factors on biofilm biomass. Seasonality greatly influenced aquatic biofilm as greater DW and AFDW was observed in the summer but greater content of PUFA (standardized for mass of organic matter) were observed in the fall. The overall yield of PUFA, however, was higher in the summer, overall and specifically at greater depths. A maximum PUFA yield observed in fall biofilm incubated at $100 \mathrm{~cm}$ may suggest an optimal combination of environmental factors, resulting in a balance between biofilm quantity and nutritional quality. Fall biofilm also had higher proportions of MUFA and PUFA, potentially indicating a temperature-induced biochemical response to colder water temperatures. Although water temperatures also differed between summer depths, a similar pattern between proportions was not observed suggesting that small differences between depths do not induce homeoviscous adaptation.

Biofilm incubated at greater depths did however have higher biomass but lower PUFA content, especially during summer months. Higher AFDW observed on deeper tiles in summer may be related to the deposition of pelagic phytoplankton, especially diatoms (indicated by the high abundance of 18:1n-9). The findings of this study supplement the current knowledge on the relationship between depth and biofilm biomass, which has varied between studies. The FA content of depth-distributed biofilm between seasons also add to the limited dataset of biofilm FA profiles. The results from this study can also provide a plausible explanation to some observations of higher grazer abundance near the water surface as this may be related to the nutritional quality of biofilm, their food source for physiologically-important PUFA. 


\subsection{Research Recommendations}

It is important to understand the physiological impact of warming temperatures on freshwater ecosystems in order to fully assess potential consequences of climate change. In-situ mesocosms are an essential tool in determine potential effects of warming temperatures in natural system as they provide a balance design between control and realism. The experimental setup discussed in Chapter 2 was designed with the purpose of measuring biochemical effects of several trophic levels from primary producers to top predators, within the limits of the pond's isolated location.

I suggest the following modifications for any future attempts to manipulate temperature of large mesocosms. Although directly circulating pond water into a heater would be a more effective method to transfer heat to the system, I would recommend using an indirect heating method so microscopic organisms (i.e., phytoplankton and zooplankton) are not exposed to extremely high temperatures. The indirect heating design (i.e., piping system) has been used in smaller mesocosms (McKee et al. 2000), however, its limited heat transfer efficiency would limit its usage in very large aquatic systems. To compensate, a shallow pond $(e . g .,<1 \mathrm{~m})$ with a smaller volume $(<300,000 \mathrm{~L})$ should be selected to ensure the entire water column can be efficiently heated. Finally, a propane heater attached to the piping system would be more efficient than an electrical heater by creating a larger temperature difference between water circulating in the pipes and the pond water, without being limited by local electrical restrictions. The objectives and methodology applied in this thesis should be applied in future mesocosm experiments in order to understand ecosystem-wide repercussions of warming temperatures.

Despite the challenges associated with large-scale mesocosms, they are important in confirming laboratory results and determining realistic consequences of warmer temperatures within the context of an entire ecosystem. Interestingly, the effect of warming temperature on aquatic biofilm has also not been fully explored. Despite their important at the base of freshwater food webs, content of physiologically-important PUFA, and several studies demonstrating that temperature may influence PUFA content in biofilm (e.g., Guo et al. 2015; Honeyfield \& Maloney 2015). An increase in MUFA and PUFA proportions in fall biofilm was also reported in Chapter 3, which highlights the importance of studying natural variation in biofilm quantity and quality, as well as incorporating biofilms into future climate change studies. 
Freshwater biofilms provide grazers with physiologically-important fatty acids (i.e., EPA and DHA) confirming their importance as a basal resource of PUFA. Season and depth were shown to influence both biomass and PUFA content, suggesting the importance of abiotic factors in determining availability of biofilm quantity and nutritional quality. Future studies that incubate biofilm using artificial surfaces should also examine the effect of grazers on the aquatic biofilm. For example, unglazed tiles should be incubated at different depths with and without grazer-proof netting through multiple seasons. Such an experiment would allow the separation of abiotic and biotic factors that influence aquatic biofilm and provide wholistic picture of natural variation in biofilm quantity and nutritional quality. Finally, the discrepancies observed between AFDW measurements with CLSM represent an opportunity for future research to determine both large- and fine-scaled changes in aquatic biofilm exposed to different treatments.

It is evident that temperature may influence the production of physiologically-important PUFA in freshwater systems. Collection of field-incubated samples and in-situ manipulation experiments represent essential tools in understanding the natural variation and potential biochemical effects of increasing water temperature, especially in a warming world. 


\section{Appendix 1: Common Abbreviations}

\begin{tabular}{|c|c|}
\hline Abbreviation & Nomenclature (Chemical structure, if applicable) \\
\hline FA & Fatty Acid \\
\hline $\mathrm{SC}$ & Short-Chain \\
\hline $\mathrm{LC}$ & Long-Chain \\
\hline SFA & Saturated Fatty Acid \\
\hline UFA & Unsaturated Fatty Acid \\
\hline MUFA & Monounsaturated Fatty Acid \\
\hline PUFA & Polyunsaturated Fatty Acid \\
\hline HUFA & Highly Unsaturated Fatty Acid \\
\hline EFA & Essential Fatty Acid \\
\hline$n-3$ & Omega-3 \\
\hline$n-6$ & Omega-6 \\
\hline EPA & Eicosapentaenoic acid (20:5n-3) \\
\hline DHA & Docosahexaenoic acid (22:6n-3) \\
\hline ARA & Arachidonic acid (20:4n-6) \\
\hline ALA & $\alpha$-Linolenic acid (18:3n-3) \\
\hline LNA & Linoleic acid (18:2n-6) \\
\hline FAME & Fatty Acid Methyl Ester \\
\hline $\mathrm{GC}$ & Gas Chromatography \\
\hline WW & Wet Weight \\
\hline DW & Dry Weight \\
\hline AFDW & Ash-Free Dry Weight \\
\hline MANOVA & Multivariate Analysis of Variance \\
\hline ANOVA & Analysis of Variance \\
\hline EPS & Extracellular Polymeric Substance \\
\hline PAR & Photosynthetically Active Radiation \\
\hline UV & Ultraviolet \\
\hline Chl $a$ & Chlorophyll $a$ \\
\hline CLSM & Confocal Laser Scanning Microscopy \\
\hline GMST & Global Mean Surface Temperature \\
\hline PVC & Polyvinyl Chloride \\
\hline HDPE & High Density Polyethylene \\
\hline UI & Unsaturation Index \\
\hline TDS & Total Dissolved Solids \\
\hline ODO & Optical Dissovled Oxygen \\
\hline CDOM & Colored Dissolved Organic Matter \\
\hline Tukey HSD & Tukey Honestly Significant Difference \\
\hline SD & Standard Deviation \\
\hline
\end{tabular}




\section{Appendix 2: Chemical Composition of Hard COMBO Media}

\begin{tabular}{|c|c|}
\hline Constituent & Final concentration (mg/liter) \\
\hline \multicolumn{2}{|l|}{ Major stocks } \\
\hline $\mathrm{CaCl}_{2} \cdot 2 \mathrm{H}_{2} \mathrm{O}$ & 110.28 \\
\hline $\mathrm{MgSO}_{4}$ & 55.45 \\
\hline $\mathrm{K}_{2} \mathrm{HPO}_{4}$ & 1.742 \\
\hline $\mathrm{NaNO}_{3}$ & 17.0 \\
\hline $\mathrm{NaHCO}_{3}$ & 126.0 \\
\hline $\mathrm{Na}_{2} \mathrm{SiO}_{3} \cdot 9 \mathrm{H}_{2} \mathrm{O}$ & 28.42 \\
\hline $\mathrm{H}_{3} \mathrm{BO}_{3}$ & 24.0 \\
\hline $\mathrm{KCl}$ & 5.96 \\
\hline \multicolumn{2}{|c|}{ Animal trace elements (ANIMATE) } \\
\hline $\mathrm{LiCl}$ & 0.31 \\
\hline $\mathrm{RbCl}$ & 0.07 \\
\hline $\mathrm{SrCl}_{2} \cdot 6 \mathrm{H}_{2} \mathrm{O}$ & 0.15 \\
\hline $\mathrm{NaBr}$ & 0.016 \\
\hline $\mathrm{KI}$ & 0.0033 \\
\hline \multicolumn{2}{|l|}{ Vitamins } \\
\hline $\mathrm{B}_{12}($ cyanocobalamin $)$ & 0.00055 \\
\hline $\operatorname{Biotin}(d$-biotin) & 0.0005 \\
\hline Thiamine (HCl) & 0.1 \\
\hline
\end{tabular}

Media components were published by Baer and Goulden (1998). 


\section{Appendix 3: Nutrient Concentration between two Mesocosms (Control and Experimental) and the Ambient Section}

\begin{tabular}{lcccc}
\hline \multicolumn{1}{c}{ Parameter } & Units & Ambient & Control & Experimental \\
\hline \hline Dissolved Inorganic Carbon & $\mathrm{mg} / \mathrm{L}$ & 34.2 & 33.3 & 32.6 \\
Total Nitrogen & $\mathrm{mg} / \mathrm{L}$ & 0.38 & 0.43 & 0.45 \\
Total Phosphorus & $\mu \mathrm{g} / \mathrm{L}$ & 12.8 & 15 & 12 \\
Chloride & $\mathrm{mg} / \mathrm{L}$ & 24.1 & 24 & 24.4 \\
Sulphate & $\mathrm{mg} / \mathrm{L}$ & 21.7 & 21.6 & 21.8 \\
Calcium & $\mathrm{mg} / \mathrm{L}$ & 56.9 & 56.8 & 56.2 \\
Magnesium & $\mathrm{mg} / \mathrm{L}$ & 14.3 & 13.5 & 14.3 \\
Sodium & $\mathrm{mg} / \mathrm{L}$ & 6.01 & 6.13 & 5.98 \\
Potassium & $\mathrm{mg} / \mathrm{L}$ & 1.24 & 1.29 & 1.38 \\
Nitrogen (NH $\left.{ }_{3}+\mathrm{NH}_{4}{ }^{+}\right)$ & $\mu \mathrm{g} / \mathrm{L}$ & 34 & 52 & 60 \\
Nitrogen (NO $\left.{ }^{-}+\mathrm{NO}_{2}{ }^{-}\right)$ & $\mu \mathrm{g} / \mathrm{L}$ & 2 & 2 & 2 \\
pH & $\mathrm{none}$ & 8.14 & 8.12 & 8.11 \\
Alkalinity (Total Fixed Endpoint) & $\mathrm{mg} / \mathrm{L} \mathrm{CaCO} 3$ & 1.54 & 1.5 & 1.57 \\
Alkalinity (Gran $\left.\mathrm{Method}^{\prime}\right)$ & $\mathrm{mg} / \mathrm{L} \mathrm{CaCO} 3$ & 1.53 & 1.49 & 1.55 \\
Conductivity & $\mu \mathrm{S} / \mathrm{cm}$ & 409 & 400 & 412 \\
Color; True & $\mathrm{TCU}$ & 1.44 & 1.8 & 1.95 \\
Total Phosphorus & $\mu \mathrm{g} / \mathrm{L}$ & 14.8 & 15.8 & 15.4 \\
\hline
\end{tabular}

Water was collected on June $20^{\text {th }}, 2017$ and all analyses done with standard Ontario Ministry of the Environment and Climate Change (OMECC) protocols. 


\section{Appendix 4: Identifiable Fatty Acids analyzed}

\begin{tabular}{lc}
\hline & Total Fatty Acids \\
\hline \hline Saturated Fatty Acids & Polyunsaturated Fatty Acids \\
$14: 0$ & $n-3$ PUFA \\
$15: 0$ & $18: 3 \mathrm{n}-3$ \\
$16: 0$ & $18: 4 \mathrm{n}-3$ \\
$17: 0$ & $20: 3 \mathrm{n}-3$ \\
$18: 0$ & $20: 5 \mathrm{n}-3$ \\
$19: 0$ & $22: 3 \mathrm{n}-3$ \\
$20: 0$ & $22: 5 \mathrm{n}-3$ \\
$22: 0$ & $22: 6 \mathrm{n}-3$ \\
$24: 0$ & \\
& \\
Monounsaturated Fatty Acids & \\
$14: 1 \mathrm{n}-5$ & $18: 2 \mathrm{n}-6 \mathrm{c}$ \\
$15: 1 \mathrm{n}-5$ & $18: 2 \mathrm{n}-6 \mathrm{t}$ \\
$16: 1 \mathrm{n}-7 \mathrm{c}$ & $20: 2 \mathrm{n}-6$ \\
$16: 1 \mathrm{n}-7 \mathrm{t}$ & $20: 3 \mathrm{n}-6$ \\
$17: 1 \mathrm{n}-7$ & $20: 4 \mathrm{n}-6$ \\
$18: 1 \mathrm{n}-7 \mathrm{c}$ & $22: 2 \mathrm{n}-6$ \\
$18: 1 \mathrm{n}-7 \mathrm{t}$ & $22: 4 \mathrm{n}-6$ \\
$18: 1 \mathrm{n}-9 \mathrm{c}$ & \\
$18: 1 \mathrm{n}-9 \mathrm{t}$ & \\
$18: 1 \mathrm{n}-12 \mathrm{c}$ & \\
$19: 1 \mathrm{n}-12$ & \\
$20: 1 \mathrm{n}-9$ & \\
$20: 1 \mathrm{n}-12$ & \\
$22: 1 \mathrm{n}-9$ & \\
$24: 1 \mathrm{n}-9$ & \\
& \\
\hline
\end{tabular}




\section{Appendix 5: Complete Statistical Summaries Conducted in Chapter 3}

Table A5.1: ANOVA on iButton residual temperature data. Bolded p-values represent significance.

\begin{tabular}{cccccc}
\hline Response & df & $\begin{array}{c}\text { Sum of } \\
\text { Squares }\end{array}$ & $\begin{array}{c}\text { Mean } \\
\text { Squares }\end{array}$ & F-value & p value \\
\hline Season & 1 & 5144329 & 5144329 & 1691000 & $<\mathbf{0 . 0 0 0 1}$ \\
Block & 2 & 84 & 42 & 13.850 & $<\mathbf{0 . 0 0 0 1}$ \\
Depth & 5 & 1682 & 336 & 110.5 & $<\mathbf{0 . 0 0 0 1}$ \\
Season $\times$ Block & 2 & 5 & 2 & 0.758 & 0.469 \\
Season $\times$ Depth & 5 & 1570 & 314 & 103.2 & $<\mathbf{0 . 0 0 0 1}$ \\
Block $\times$ Depth & 10 & 144 & 14 & 4.719 & $<\mathbf{0 . 0 0 0 1}$ \\
Residuals & 114094 & 347105 & 3 & & \\
\hline
\end{tabular}


Table A5.2: (A) MANOVA on Z-scores parameters in YSI dataset and individual ANOVA on (B) water temperature, (C), conductivity, (D) TDS, (E) pH. (F) ODO, and (G) CDOM. Bolded pvalues represent significance.

\begin{tabular}{|c|c|c|c|c|c|c|}
\hline Response & $\mathrm{df}$ & $\begin{array}{c}\text { Pillai's } \\
\text { Trace }\end{array}$ & F-value & Num & Den & $\mathrm{p}$ value \\
\hline \multicolumn{7}{|l|}{ (A) } \\
\hline Season & 1 & 0.971 & 4624.9 & 6 & 834 & $<0.0001$ \\
\hline Block & 2 & 0.013 & 0.9 & 12 & 1670 & 0.547 \\
\hline Depth & 5 & 0.344 & 10.3 & 30 & 4190 & $<0.0001$ \\
\hline Season $\times$ Block & 2 & 0.015 & 1.1 & 12 & 1670 & 0.376 \\
\hline Season $\times$ Depth & 5 & 0.267 & 7.9 & 30 & 4190 & $<0.0001$ \\
\hline Block $\times$ Depth & 10 & 0.079 & 1.1 & 60 & 5034 & 0.256 \\
\hline Residuals & 839 & & & & & \\
\hline Response & df & $\begin{array}{l}\text { Sum of } \\
\text { Squares }\end{array}$ & $\begin{array}{c}\text { Mean } \\
\text { Squares }\end{array}$ & F-value & & $\mathrm{p}$ value \\
\hline \multicolumn{7}{|l|}{ (B) } \\
\hline Season & 1 & 3682 & 3682 & 3274.866 & & $<0.0001$ \\
\hline Block & 2 & 0 & 0 & 0.009 & & 0.991 \\
\hline Depth & 5 & 0 & 0 & 0.030 & & 1.000 \\
\hline Season $\times$ Block & 2 & 0 & 0 & 0.014 & & 0.986 \\
\hline Season $\times$ Depth & 5 & 1 & 0 & 0.221 & & 0.954 \\
\hline Block $\times$ Depth & 10 & 0 & 0 & 0.005 & & 1.000 \\
\hline Residuals & 839 & 943 & 1 & & & \\
\hline \multicolumn{7}{|l|}{ (C) Cond } \\
\hline Season & 1 & $4.02 \mathrm{E}+27$ & $4.02 \mathrm{E}+27$ & 15020.806 & & $<0.0001$ \\
\hline Block & 2 & $4.20 \mathrm{E}+22$ & $2.10 \mathrm{E}+22$ & 0.078 & & 0.925 \\
\hline Depth & 5 & $6.89 \mathrm{E}+22$ & $1.38 \mathrm{E}+22$ & 0.051 & & 0.998 \\
\hline Season $\times$ Block & 2 & $1.75 \mathrm{E}+23$ & $8.77 \mathrm{E}+22$ & 0.327 & & 0.721 \\
\hline Season $\times$ Depth & 5 & $3.20 \mathrm{E}+22$ & $6.40 \mathrm{E}+21$ & 0.024 & & 1.000 \\
\hline Block $\times$ Depth & 10 & $1.07 \mathrm{E}+23$ & $1.07 \mathrm{E}+22$ & 0.040 & & 1.000 \\
\hline Residuals & 839 & $2.25 E+26$ & $2.68 \mathrm{E}+23$ & & & \\
\hline \multicolumn{7}{|l|}{ (D) $\mathrm{TDS}$} \\
\hline Season & 1 & $6.43 \mathrm{E}+25$ & $6.43 \mathrm{E}+25$ & 15560.446 & & $<0.0001$ \\
\hline Block & 2 & $3.87 \mathrm{E}+20$ & $1.94 \mathrm{E}+20$ & 0.047 & & 0.954 \\
\hline Depth & 5 & $1.09 \mathrm{E}+21$ & $2.18 \mathrm{E}+20$ & 0.053 & & 0.998 \\
\hline Season $\times$ Block & 2 & $4.56 \mathrm{E}+21$ & $2.28 \mathrm{E}+21$ & 0.552 & & 0.576 \\
\hline Season $\times$ Depth & 5 & $1.30 \mathrm{E}+21$ & $2.60 \mathrm{E}+20$ & 0.063 & & 0.997 \\
\hline Block $\times$ Depth & 10 & $2.14 \mathrm{E}+21$ & $2.14 \mathrm{E}+20$ & 0.052 & & 1.000 \\
\hline Residuals & 839 & $3.47 \mathrm{E}+24$ & $4.131 \mathrm{e}+21$ & & & \\
\hline
\end{tabular}

(Continued) 


\begin{tabular}{|c|c|c|c|c|c|}
\hline Response & df & $\begin{array}{c}\text { Sum of } \\
\text { Squares }\end{array}$ & $\begin{array}{c}\text { Mean } \\
\text { Squares }\end{array}$ & F-value & $\mathrm{p}$ value \\
\hline \multicolumn{6}{|l|}{ (E) } \\
\hline Season & 1 & $7.31 \mathrm{E}+61$ & $7.31 \mathrm{E}+61$ & 306.336 & $<0.0001$ \\
\hline Block & 2 & $4.30 \mathrm{E}+59$ & $2.15 \mathrm{E}+59$ & 0.901 & 0.406 \\
\hline Depth & 5 & $1.49 \mathrm{E}+60$ & $2.97 \mathrm{E}+59$ & 1.244 & 0.286 \\
\hline Season $\times$ Block & 2 & $8.47 \mathrm{E}+59$ & $4.24 \mathrm{E}+59$ & 1.775 & 0.170 \\
\hline Season $\times$ Depth & 5 & $9.06 \mathrm{E}+59$ & $1.81 \mathrm{E}+59$ & 0.760 & 0.579 \\
\hline Block $\times$ Depth & 10 & $1.42 \mathrm{E}+59$ & $1.42 \mathrm{E}+58$ & 0.059 & 1.000 \\
\hline Residuals & 839 & $2.00 \mathrm{E}+62$ & $2.39 \mathrm{E}+59$ & & \\
\hline \multicolumn{6}{|l|}{ (F) } \\
\hline Season & 1 & 12823 & 12823 & 1294.261 & $<0.0001$ \\
\hline Block & 2 & 4 & 2 & 0.189 & 0.827 \\
\hline Depth & 5 & 4 & 1 & 0.074 & 0.996 \\
\hline Season $\times$ Block & 2 & 3 & 2 & 0.176 & 0.839 \\
\hline Season $\times$ Depth & 5 & 4 & 1 & 0.088 & 0.994 \\
\hline Block $\times$ Depth & 9 & 2 & 0 & 0.024 & 1.000 \\
\hline Residuals & 838 & 8303 & 10 & & \\
\hline \multicolumn{6}{|l|}{ (G) } \\
\hline Season & 1 & 794.7 & 794.7 & 779.255 & $<0.0001$ \\
\hline Block & 2 & 3.9 & 2.0 & 1.915 & 0.148 \\
\hline Depth & 5 & 87.5 & 17.5 & 17.158 & $<0.0001$ \\
\hline Season $\times$ Block & 2 & 0.5 & 0.2 & 0.236 & 0.790 \\
\hline Season $\times$ Depth & 5 & 50.1 & 10.0 & 9.823 & $<0.0001$ \\
\hline Block $\times$ Depth & 9 & 9.4 & 1.0 & 1.027 & 0.416 \\
\hline Residuals & 838 & 854.6 & 1.0 & & \\
\hline
\end{tabular}


Table A5.3: (A) MANOVA on underwater light dataset and individual ANOVA on (B) PAR, (C), UV-R, (D) UV-A, and (E) UV-B. Bolded and " $\uparrow$ " p-values represent significance and marginally significant, respectively.

\begin{tabular}{|c|c|c|c|c|c|c|}
\hline Response & df & $\begin{array}{l}\text { Pillai's } \\
\text { Trace }\end{array}$ & F-value & Num & Den & $\mathrm{p}$ value \\
\hline \multicolumn{7}{|l|}{ (A) } \\
\hline Season & 1 & 0.828 & 1494.18 & 4 & 1245 & $<0.0001$ \\
\hline Depth & 5 & 1.241 & 112.25 & 20 & 4992 & $<0.0001$ \\
\hline Season $\times$ Depth & 5 & 0.232 & 15.34 & 20 & 4992 & $<0.0001$ \\
\hline Residuals & 1248 & & & & & \\
\hline Response & df & $\begin{array}{l}\text { Sum of } \\
\text { Squares }\end{array}$ & $\begin{array}{c}\text { Mean } \\
\text { Squares }\end{array}$ & F-value & \multicolumn{2}{|c|}{$\mathrm{p}$ value } \\
\hline \multicolumn{7}{|l|}{ (B) } \\
\hline Season & 1 & 1926.39 & 1926.39 & 1630.275 & \multicolumn{2}{|c|}{$<0.0001$} \\
\hline Depth & 5 & 424.08 & 84.82 & 71.779 & \multirow{2}{*}{\multicolumn{2}{|c|}{$\begin{array}{c}<\mathbf{0 . 0 0 0 1} \\
0.074^{\dagger}\end{array}$}} \\
\hline Season $\times$ Depth & 5 & 11.88 & 2.38 & 2.011 & & \\
\hline Residuals & 1248 & 1474.68 & 1.18 & & & \\
\hline \multicolumn{7}{|l|}{ (C) } \\
\hline Season & 1 & 210.57 & 210.573 & 901.469 & \multicolumn{2}{|c|}{$<0.0001$} \\
\hline Depth & 5 & 636.81 & 127.361 & 545.238 & \multirow{2}{*}{\multicolumn{2}{|c|}{$\begin{array}{c}<\mathbf{0 . 0 0 0 1} \\
0.494\end{array}$}} \\
\hline Season $\times$ Depth & 5 & 1.03 & 0.205 & 0.879 & & \\
\hline Residuals & 1248 & 291.52 & 0.234 & & & \\
\hline \multicolumn{7}{|l|}{ (D) } \\
\hline Season & 1 & 236.35 & 236.347 & 1028.748 & \multicolumn{2}{|c|}{$<0.0001$} \\
\hline Depth & 5 & 748.37 & 149.673 & 651.483 & \multirow{2}{*}{\multicolumn{2}{|c|}{$\begin{array}{c}<\mathbf{0 . 0 0 0 1} \\
0.822\end{array}$}} \\
\hline Season $\times$ Depth & 5 & 0.5 & 0.101 & 0.4381 & & \\
\hline Residuals & 1248 & 286.72 & 0.230 & & & \\
\hline \multicolumn{7}{|l|}{ (E) } \\
\hline Season & 1 & 6.562 & 6.562 & 272.047 & \multicolumn{2}{|c|}{$<0.0001$} \\
\hline Depth & 5 & 12.399 & 2.480 & 102.805 & \multirow{2}{*}{\multicolumn{2}{|c|}{$\begin{array}{c}<0.0001 \\
<0.01\end{array}$}} \\
\hline Season $\times$ Depth & 5 & 0.393 & 0.079 & 3.256 & & \\
\hline Residuals & 1248 & 30.103 & 0.024 & & & \\
\hline
\end{tabular}


Table A5.4: (A) MANOVA on Z-scores parameters in CLSM dataset and individual ANOVA on (B) bio-volume, (C) mean thickness, and (D) roughness coefficient. Bolded and "i”" p-values represent significance and marginally significant, respectively.

\begin{tabular}{|c|c|c|c|c|c|c|}
\hline Response & df & $\begin{array}{c}\text { Pillai's } \\
\text { Trace }\end{array}$ & F-value & Num & Den & $\mathrm{p}$ value \\
\hline \multicolumn{7}{|l|}{ (A) } \\
\hline Season & 1 & 0.538 & 95.035 & 4 & 326 & $<0.0001$ \\
\hline Block & 2 & 0.195 & 8.830 & 8 & 654 & $<0.0001$ \\
\hline Depth & 5 & 0.596 & 11.510 & 20 & 1316 & $<0.0001$ \\
\hline Season $\times$ Block & 2 & 0.281 & 13.353 & 8 & 654 & $<0.0001$ \\
\hline Season $\times$ Depth & 5 & 0.338 & 6.074 & 20 & 1316 & $<0.0001$ \\
\hline Block $\times$ Depth & 10 & 0.275 & 2.430 & 40 & 1316 & $<0.0001$ \\
\hline Residuals & 329 & & & & & \\
\hline Response & df & $\begin{array}{l}\text { Sum of } \\
\text { Squares }\end{array}$ & $\begin{array}{c}\text { Mean } \\
\text { Squares }\end{array}$ & F-value & & $\mathrm{p}$ value \\
\hline \multicolumn{7}{|l|}{ (B) } \\
\hline Season & 1 & 0.770 & 0.773 & 2.251 & & 0.134 \\
\hline Block & 2 & 9.830 & 4.913 & 14.306 & & $<0.0001$ \\
\hline Depth & 5 & 78.110 & 15.623 & 45.489 & & $<0.0001$ \\
\hline Season $\times$ Block & 2 & 18.110 & 9.055 & 26.365 & & $<0.0001$ \\
\hline Season $\times$ Depth & 5 & 4.100 & 0.820 & 2.386 & & 0.038 \\
\hline Block $\times$ Depth & 10 & 17.560 & 1.756 & 5.114 & & $<0.0001$ \\
\hline Residuals & 329 & 113.0 & 0.343 & & & \\
\hline \multicolumn{7}{|l|}{ (C) } \\
\hline Season & 1 & 135.700 & 135.740 & 15.694 & & $<0.0001$ \\
\hline Block & 2 & 132.400 & 66.190 & 7.654 & & $<0.001$ \\
\hline Depth & 5 & 1524.300 & 304.860 & 35.249 & & $<0.0001$ \\
\hline Season $\times$ Block & 2 & 53.500 & 26.740 & 3.092 & & 0.047 \\
\hline Season $\times$ Depth & 5 & 163.800 & 32.750 & 3.787 & & $<0.01$ \\
\hline Block $\times$ Depth & 10 & 228.200 & 22.820 & 2.639 & & $<0.01$ \\
\hline Residuals & 329 & 2845.5 & 8.650 & & & \\
\hline \multicolumn{7}{|l|}{ (D) } \\
\hline Season & 1 & 0.071 & 0.071 & 1.802 & & 0.180 \\
\hline Block & 2 & 0.802 & 0.401 & 10.137 & & $<0.0001$ \\
\hline Depth & 5 & 9.979 & 1.996 & 50.478 & & $<0.0001$ \\
\hline Season $\times$ Block & 2 & 1.229 & 0.615 & 15.546 & & $<0.0001$ \\
\hline Season $\times$ Depth & 5 & 0.606 & 0.121 & 3.067 & & $<0.01$ \\
\hline Block $\times$ Depth & 10 & 1.579 & 0.158 & 3.993 & & $<0.0001$ \\
\hline Residuals & 329 & 13.0 & 0.040 & & & \\
\hline
\end{tabular}


Table A5.5: Individual ANOVA on (A) dry weights (B) moisture content, (C) AFDW, and (D) organic percentage. Bolded and "i" p-values represent significance and marginally significant, respectively.

\begin{tabular}{|c|c|c|c|c|c|}
\hline Response & df & $\begin{array}{l}\text { Sum of } \\
\text { Squares }\end{array}$ & $\begin{array}{c}\text { Mean } \\
\text { Squares }\end{array}$ & F-value & $\mathrm{p}$ value \\
\hline \multicolumn{6}{|l|}{ (A) } \\
\hline Season & 1 & 0.001458 & 0.001458 & 482.422 & $<0.0001$ \\
\hline Block & 2 & 0.000049 & 0.000024 & 8.053 & $<0.001$ \\
\hline Depth & 5 & 0.000459 & 0.000092 & 30.361 & $<0.0001$ \\
\hline Season $\times$ Block & 2 & 0.000010 & 0.000005 & 1.624 & 0.203 \\
\hline Season $\times$ Depth & 5 & 0.000448 & 0.000090 & 29.633 & $<0.0001$ \\
\hline Block $\times$ Depth & 10 & 0.000051 & 0.000005 & 1.698 & $0.095^{\dagger}$ \\
\hline Residuals & 82 & 0.000248 & 0.000003 & & \\
\hline \multicolumn{6}{|l|}{ (B) } \\
\hline Season & 1 & $1.33 \mathrm{E}+40$ & $1.33 \mathrm{E}+40$ & 1693.757 & $<0.0001$ \\
\hline Block & 2 & $4.50 \mathrm{E}+38$ & $2.25 \mathrm{E}+38$ & 28.615 & $<0.0001$ \\
\hline Depth & 5 & $1.15 \mathrm{E}+39$ & $2.31 \mathrm{E}+38$ & 29.361 & $<0.0001$ \\
\hline Season $\times$ Block & 2 & $1.59 \mathrm{E}+38$ & $7.97 \mathrm{E}+37$ & 10.132 & $<0.001$ \\
\hline Season $\times$ Depth & 5 & $1.37 E+39$ & $2.74 \mathrm{E}+38$ & 34.810 & $<0.0001$ \\
\hline Block $\times$ Depth & 10 & $2.78 E+38$ & $2.78 \mathrm{E}+37$ & 3.532 & $<0.001$ \\
\hline Residuals & 82 & $6.45 E+38$ & $7.86 \mathrm{E}+36$ & & \\
\hline \multicolumn{6}{|l|}{ (C) } \\
\hline Season & 1 & $1.85 \mathrm{E}-05$ & $1.85 \mathrm{E}-05$ & 121.468 & $<0.0001$ \\
\hline Block & 2 & $1.13 \mathrm{E}-07$ & $5.60 \mathrm{E}-08$ & 0.370 & 0.70 \\
\hline Depth & 5 & $1.16 \mathrm{E}-05$ & $2.32 \mathrm{E}-06$ & 15.258 & $<0.0001$ \\
\hline Season $\times$ Block & 2 & $8.85 \mathrm{E}-07$ & $4.43 \mathrm{E}-07$ & 2.907 & 0.101 \\
\hline Season $\times$ Depth & 5 & $9.23 \mathrm{E}-06$ & $1.85 \mathrm{E}-06$ & 12.122 & $<0.001$ \\
\hline Block $\times$ Depth & 10 & $1.33 \mathrm{E}-06$ & $1.33 \mathrm{E}-07$ & 0.874 & 0.58 \\
\hline Residuals & 10 & $1.52 \mathrm{E}-06$ & $1.52 \mathrm{E}-07$ & & \\
\hline \multicolumn{6}{|l|}{ (D) } \\
\hline Season & 1 & 0.014831 & 0.014831 & 600.384 & $<0.0001$ \\
\hline Block & 2 & 0.002628 & 0.001314 & 53.197 & $<0.0001$ \\
\hline Depth & 5 & 0.000343 & 0.000069 & 2.774 & $0.080^{\dagger}$ \\
\hline Season $\times$ Block & 2 & 0.000299 & 0.000150 & 6.056 & 0.019 \\
\hline Season $\times$ Depth & 5 & 0.001425 & 0.000285 & 11.536 & $<0.001$ \\
\hline Block $\times$ Depth & 10 & 0.000511 & 0.000051 & 2.071 & 0.133 \\
\hline Residuals & 10 & 0.000247 & 0.000025 & & \\
\hline
\end{tabular}


Table A5.6: (A) MANOVA on summer nutritional quality parameters individual ANOVA on (B) $\sum$ n-3 PUFA, (C) $\sum$ n-6 PUFA, and (D) EPA+DHA content. Individual ANOVA are also presented for (E) 16:1n-7 and (F) $\sum$ Odd-chained FA. Bolded p-values represent significance.

\begin{tabular}{|c|c|c|c|c|c|c|}
\hline Response & $\mathrm{df}$ & $\begin{array}{c}\text { Pillai's } \\
\text { Trace }\end{array}$ & F-value & Num & Den & $\mathrm{p}$ value \\
\hline \multicolumn{7}{|l|}{ (A) } \\
\hline Block & 2 & 0.681 & 1.549 & 6 & 18 & 0.219 \\
\hline Depth & 5 & 1.737 & 2.749 & 15 & 30 & $<0.01$ \\
\hline Residuals & 10 & & & & & \\
\hline Response & $\mathrm{df}$ & $\begin{array}{l}\text { Sum of } \\
\text { Squares }\end{array}$ & $\begin{array}{c}\text { Mean } \\
\text { Squares }\end{array}$ & F-value & \multicolumn{2}{|r|}{$\mathrm{p}$ value } \\
\hline \multicolumn{7}{|l|}{ (B) } \\
\hline Block & 2 & 0.004 & 0.002 & 0.005 & \multirow{2}{*}{\multicolumn{2}{|c|}{$\begin{array}{l}0.995 \\
<\mathbf{0 . 0 1}\end{array}$}} \\
\hline Depth & 5 & 18.978 & 3.796 & 8.891 & & \\
\hline Residuals & 10 & 4.269 & 0.427 & & & \\
\hline \multicolumn{7}{|l|}{ (C) } \\
\hline Block & 2 & 0.073 & 0.037 & 1.696 & \multirow{2}{*}{\multicolumn{2}{|c|}{$\begin{array}{l}0.232 \\
\mathbf{0 . 0 3 1}\end{array}$}} \\
\hline Depth & 5 & 0.425 & 0.085 & 3.954 & & \\
\hline Residuals & 10 & 0.2152 & 0.02152 & & & \\
\hline \multicolumn{7}{|l|}{ (D) } \\
\hline Block & 2 & 0.005 & 0.002 & 0.010 & \multirow{3}{*}{\multicolumn{2}{|c|}{$\begin{array}{c}0.990 \\
<\mathbf{0 . 0 0 1}\end{array}$}} \\
\hline Depth & 5 & 13.493 & 2.699 & 12.074 & & \\
\hline Residuals & 10 & 2.235 & 0.224 & & & \\
\hline \multicolumn{7}{|l|}{ (E) } \\
\hline Block & 2 & 2.119 & 1.060 & 8.296 & \multirow{3}{*}{\multicolumn{2}{|c|}{$\begin{array}{c}<0.01 \\
<0.0001\end{array}$}} \\
\hline Depth & 5 & 15.901 & 3.180 & 24.900 & & \\
\hline Residuals & 10 & 1.277 & 0.128 & & & \\
\hline \multicolumn{7}{|l|}{ (F) } \\
\hline Block & 2 & 0.002 & 0.001 & 5.127 & \multirow{3}{*}{\multicolumn{2}{|c|}{$\begin{array}{c}0.029 \\
<0.001\end{array}$}} \\
\hline Depth & 5 & 0.012 & 0.002 & 10.878 & & \\
\hline Residuals & 10 & 0.002 & 0.0002 & & & \\
\hline
\end{tabular}


Table A5.7: (A) MANOVA on fall nutritional quality parameters individual ANOVA on (B) $\sum$ n-3 PUFA, (C) $\sum$ n-6 PUFA, and (D) EPA+DHA content. Individual ANOVA are also presented for (E) 16:1n-7 and (F) $\sum$ Odd-chained FA. Bolded and “ $\dagger$ " p-values represent significance and marginally significant, respectively.

\begin{tabular}{|c|c|c|c|c|c|c|}
\hline Response & $\mathrm{df}$ & $\begin{array}{c}\text { Pillai's } \\
\text { Trace } \\
\end{array}$ & F-value & Num & Den & $\mathrm{p}$ value \\
\hline \multicolumn{7}{|l|}{ (A) } \\
\hline Block & 2 & 1.510 & 9.252 & 6 & 18 & $<0.001$ \\
\hline Depth & 5 & 1.398 & 1.745 & 15 & 30 & $0.095^{\dagger}$ \\
\hline Residuals & 10 & & & & & \\
\hline Response & df & $\begin{array}{l}\text { Sum of } \\
\text { Squares }\end{array}$ & $\begin{array}{c}\text { Mean } \\
\text { Squares }\end{array}$ & F-value & \multicolumn{2}{|r|}{$\mathrm{p}$ value } \\
\hline \multicolumn{7}{|l|}{ (B) } \\
\hline Block & 2 & 3.605 & 1.803 & 3.976 & \multirow{3}{*}{\multicolumn{2}{|c|}{$\begin{array}{c}0.054^{\dagger} \\
0.320\end{array}$}} \\
\hline Depth & 5 & 3.063 & 0.613 & 1.351 & & \\
\hline Residuals & 10 & 4.534 & 0.4534 & & & \\
\hline \multicolumn{7}{|l|}{ (C) } \\
\hline Block & 2 & 0.070 & 0.035 & 1.073 & \multirow{3}{*}{\multicolumn{2}{|c|}{$\begin{array}{l}0.378 \\
<\mathbf{0 . 0 1}\end{array}$}} \\
\hline Depth & 5 & 1.492 & 0.298 & 9.128 & & \\
\hline Residuals & 10 & 0.327 & 0.033 & & & \\
\hline \multicolumn{7}{|l|}{ (D) } \\
\hline Block & 2 & 2.101 & 1.050 & 6.541 & \multirow{3}{*}{\multicolumn{2}{|c|}{$\begin{array}{l}0.015 \\
0.024\end{array}$}} \\
\hline Depth & 5 & 3.452 & 0.690 & 4.298 & & \\
\hline Residuals & 10 & 1.606 & 0.161 & & & \\
\hline \multicolumn{7}{|l|}{ (E) } \\
\hline Block & 2 & 8.127 & 4.064 & 4.006 & \multirow{3}{*}{\multicolumn{2}{|c|}{$\begin{array}{c}0.053^{\dagger} \\
\mathbf{0 . 0 4 4}\end{array}$}} \\
\hline Depth & 5 & 17.685 & 3.537 & 3.487 & & \\
\hline Residuals & 10 & 10.143 & 1.014 & & & \\
\hline \multicolumn{7}{|l|}{ (F) } \\
\hline Block & 2 & 0.007 & 0.003 & 0.219 & \multirow{3}{*}{\multicolumn{2}{|c|}{$\begin{array}{l}0.807 \\
0.769\end{array}$}} \\
\hline Depth & 5 & 0.037 & 0.007 & 0.502 & & \\
\hline Residuals & 10 & 0.148 & 0.015 & & & \\
\hline
\end{tabular}


Table A5.8: (A) MANOVA on summer summary indices parameters and individual ANOVA on (B) $\sum$ FA, (C) $\sum$ SFA, (D) $\sum$ MUFA, (E) $\sum$ PUFA. Individual ANOVA were included for (F) UI, (G) 18:1n-9 content, and (H) n3:n6 ratio. B Bolded and " $\uparrow$ " p-values represent significance and marginally significant, respectively.

\begin{tabular}{|c|c|c|c|c|c|c|}
\hline Response & $\mathrm{df}$ & Pillai's Trace & F-value & Num & Den & $\mathrm{p}$ value \\
\hline \multicolumn{7}{|l|}{ (A) } \\
\hline Block & 2 & 1.404 & 2.355 & 12 & 12 & $0.076^{\dagger}$ \\
\hline Depth & 5 & 3.145 & 2.544 & 30 & 45 & $<0.01$ \\
\hline Residuals & 10 & & & & & \\
\hline Response & df & $\begin{array}{l}\text { Sum of } \\
\text { Squares }\end{array}$ & $\begin{array}{c}\text { Mean } \\
\text { Squares }\end{array}$ & F-value & & $\mathrm{p}$ value \\
\hline \multicolumn{7}{|l|}{ (B) } \\
\hline Block & 2 & 6.830 & 3.417 & 1.256 & & 0.326 \\
\hline Depth & 5 & 147.730 & 29.547 & 10.863 & & $<0.001$ \\
\hline Residuals & 10 & 27.200 & 2.720 & & & \\
\hline \multicolumn{7}{|l|}{ (C) } \\
\hline Block & 2 & 1.665 & 0.832 & 4.153 & & 0.049 \\
\hline Depth & 5 & 10.381 & 2.076 & 10.359 & & $<0.01$ \\
\hline Residuals & 10 & 2.004 & 0.200 & & & \\
\hline \multicolumn{7}{|l|}{ (D) } \\
\hline Block & 2 & 2.812 & 1.406 & 6.050 & & 0.019 \\
\hline Depth & 5 & 18.140 & 3.628 & 15.612 & & $<0.001$ \\
\hline Residuals & 10 & 2.324 & 0.232 & & & \\
\hline \multicolumn{7}{|l|}{ (E) } \\
\hline Block & 2 & 0.109 & 0.055 & 0.088 & & 0.916 \\
\hline Depth & 5 & 24.178 & 4.836 & 7.804 & & $<0.01$ \\
\hline Residuals & 10 & 6.196 & 0.620 & & & \\
\hline \multicolumn{7}{|l|}{ (F) } \\
\hline Block & 2 & 1.380 & 0.689 & 0.047 & & 0.954 \\
\hline Depth & 5 & 744.950 & 148.989 & 10.212 & & $<0.01$ \\
\hline Residuals & 10 & 145.890 & 14.589 & & & \\
\hline \multicolumn{7}{|l|}{ (G) } \\
\hline Block & 2 & 0.044 & 0.022 & 4.045 & & $0.052^{\dagger}$ \\
\hline Depth & 5 & 0.156 & 0.031 & 5.771 & & $<0.01$ \\
\hline Residuals & 10 & 0.054 & 0.005 & & & \\
\hline \multicolumn{7}{|l|}{ (H) } \\
\hline Block & 2 & 0.048 & 0.024 & 1.321 & & 0.310 \\
\hline Depth & 5 & 1.214 & 0.243 & 13.346 & & $<0.001$ \\
\hline Residuals & 10 & 0.182 & 0.018 & & & \\
\hline
\end{tabular}


Table A5.9: (A) MANOVA on fall summary indices parameters and individual ANOVA on (B) $\sum \mathrm{FA},(\mathrm{C}) \sum \mathrm{SFA}$, (D) $\sum$ MUFA, (E) $\sum$ PUFA. Individual ANOVA were included for (F) UI, (G) 18:1n-9 content, and (H) n3:n6 ratio. Bolded and "†" p-values represent significance and marginally significant, respectively.

\begin{tabular}{|c|c|c|c|c|c|c|}
\hline Response & $\mathrm{df}$ & Pillai's Trace & F-value & Num & Den & $\mathrm{p}$ value \\
\hline \multicolumn{7}{|l|}{ (A) } \\
\hline Block & 2 & 1.649 & 4.694 & 12 & 12 & $<0.01$ \\
\hline Depth & 5 & 2.603 & 1.629 & 30 & 45 & $0.068^{\dagger}$ \\
\hline Residuals & 10 & & & & & \\
\hline Response & df & $\begin{array}{l}\text { Sum of } \\
\text { Squares }\end{array}$ & $\begin{array}{c}\text { Mean } \\
\text { Squares } \\
\end{array}$ & F-value & \multicolumn{2}{|r|}{$\mathrm{p}$ value } \\
\hline \multicolumn{7}{|l|}{ (B) } \\
\hline Block & 2 & 40.820 & 20.410 & 2.204 & \multirow{2}{*}{\multicolumn{2}{|c|}{0.161}} \\
\hline Depth & 5 & 171.680 & 34.340 & 3.708 & & \\
\hline Residuals & 10 & 92.610 & 9.260 & & & \\
\hline \multicolumn{7}{|l|}{ (C) } \\
\hline Block & 2 & 2.713 & 1.356 & 1.868 & \multicolumn{2}{|r|}{0.205} \\
\hline Depth & 5 & 17.984 & 3.597 & 4.953 & \multicolumn{2}{|r|}{0.015} \\
\hline Residuals & 10 & 7.262 & 0.726 & & & \\
\hline \multicolumn{7}{|l|}{ (D) } \\
\hline Block & 2 & 8.994 & 4.497 & 2.329 & \multirow{2}{*}{\multicolumn{2}{|c|}{$\begin{array}{l}0.148 \\
\mathbf{0 . 0 2 6}\end{array}$}} \\
\hline Depth & 5 & 40.328 & 8.066 & 4.177 & & \\
\hline Residuals & 10 & 19.311 & 1.931 & & & \\
\hline \multicolumn{7}{|l|}{ (E) } \\
\hline Block & 2 & 4.679 & 2.339 & 3.273 & \multirow{3}{*}{\multicolumn{2}{|c|}{$\begin{array}{c}0.081^{\dagger} \\
0.137\end{array}$}} \\
\hline Depth & 5 & 7.810 & 1.562 & 2.185 & & \\
\hline Residuals & 10 & 7.148 & 0.715 & & & \\
\hline \multicolumn{7}{|l|}{ (F) } \\
\hline Block & 2 & 148.790 & 74.395 & 3.124 & \multirow{3}{*}{\multicolumn{2}{|c|}{$\begin{array}{c}0.088^{\dagger} \\
0.243\end{array}$}} \\
\hline Depth & 5 & 191.810 & 38.363 & 1.611 & & \\
\hline Residuals & 10 & 238.130 & 23.814 & & & \\
\hline \multicolumn{7}{|l|}{ (G) } \\
\hline Block & 2 & 0.824 & 0.412 & 3.635 & \multirow{3}{*}{\multicolumn{2}{|c|}{$\begin{array}{c}0.065^{\dagger} \\
<\mathbf{0 . 0 1}\end{array}$}} \\
\hline Depth & 5 & 4.993 & 0.999 & 8.817 & & \\
\hline Residuals & 10 & 1.133 & 0.113 & & & \\
\hline \multicolumn{7}{|l|}{ (H) } \\
\hline Block & 2 & 0.030 & 0.015 & 1.016 & \multirow{3}{*}{\multicolumn{2}{|c|}{$\begin{array}{c}0.397 \\
<\mathbf{0 . 0 0 0 1}\end{array}$}} \\
\hline Depth & 5 & 3.355 & 0.671 & 45.804 & & \\
\hline Residuals & 10 & 0.147 & 0.015 & & & \\
\hline
\end{tabular}




\section{References}

Abe, S., T. Nagumo, and J. Tanaka. 2000. Effects of current on the development of loosely and tightly attached layers in periphyton communities. Phycol. Res. 48: 261-265.

Abedian Kennari, A. M., M. R. Oveisipour, and R. M. Nazari. 2007. Effects of n3-HUFA enriched Daphnia magna on growth, survival, stress resistance, and fatty acid composition of larvae of Persian sturgeon (Acipenser persicus). Iran. J. Fish. Sci. 7: 1-14.

Ackman, R. G., C. S. Tocher, and J. McLachlan. 1968. Marine phytoplankter fatty acids. J. Fish. Board Canada 25: 1603-1620.

Acuña, V., A. Wolf, U. Uehlinger, and K. Tockner. 2008. Temperature dependence of stream benthic respiration in an Alpine river network under global warming. Freshw. Biol. 53: 2076-2088.

Admiraal, W. 1976. Influence of light and temperature on the growth rate of estuarine benthic diatoms in culture. Mar. Biol. 39: 1-9.

Admiraal, W., H. Peletier, and T. Brouwer. 1984. The seasonal succession patterns of diatom species on an intertidal mudflat: an experimental analysis. Oikos 42: 30-40.

Ahlgren, G., I. Gustafsson, and M. Boberg. 1992. Fatty Acid Content and Chemical Composition of Freshwater Microalgae. J. Phycol. 28: 37-50.

Aizaki, M. 1979. Growth rates of microorganisms in a periphyton community. Japanese J. Limnol. (Rikusuigaku Zasshi) 40: 10-19.

Albers, S. J. 2010. The salmon disturbance regime: Effects on biofilm, sediment and water. MSc Thesis. University of Northern British Columbia.

Alvarez, M. J., A. Diez, C. Lopez-Bote, M. Gallego, and J. M. Bautista. 2000. Short-term modulation of lipogenesis by macronutrients in rainbow trout (Oncorhynchus mykiss) hepatocytes. Br. J. Nutr. 84: 619-628.

Ancion, P. Y., G. Lear, M. Neale, K. Roberts, and G. D. Lewis. 2014. Using biofilm as a novel approach to assess stormwater treatment efficacy. Water Res. 49: 406-415.

Andrews, J. S., S. A. Rolfe, W. E. Huang, J. D. Scholes, and S. A. Banwart. 2010. Biofilm formation in environmental bacteria is influenced by different macromolecules depending on genus and species. Environ. Microbiol. 12: 2496-2507.

Anesi, A., U. Obertegger, G. Hansen, A. Sukenik, G. Flaim, and G. Guella. 2016. Comparative analysis of membrane lipids in psychrophilic and mesophilic freshwater dinoflagellates. Front. Plant Sci. 7: 524.

Antia, N. J., P. J. Harrison, and L. Oliveira. 1991. The role of dissolved organic nitrogen in phytoplankton nutrition, cell biology and ecology. Phycologia 30: 1-89.

Arnason, B., P. Theodorsson, S. Björnsson, and K. Saemundsson. 1969. Hengill, a high temperature thermal area in Iceland. Bull. Volcanol. 33: 245-259.

Arnon, S., A. I. Packman, C. G. Peterson, and K. A. Gray. 2007. Effects of overlying velocity on periphyton structure and denitrification. J. Geophys. Res. Biogeosciences 112: G01002.

Arrhenius, S. 1896. On the influence of carbonic acid in the air upon the temperature of the ground. London, Edinburgh, Dublin Philos. Mag. J. Sci. 41: 237-276.

Arts, M. T., M. T. Brett, and M. J. Kainz. 2009. Lipids in aquatic ecosystems, $2^{\text {nd }}$ ed. Springer.

Augspurger, C., G. Gleixner, C. Kramer, and K. Küsel. 2008. Tracking carbon flow in a 2-weekold and 6-week-old stream biofilm food web. Limnol. Oceanogr. 53: 642-650.

Augustine, N., P. Kumar, and S. Thomas. 2010. Inhibition of Vibrio cholerae biofilm by AiiA enzyme produced from Bacillus spp. Arch. Microbiol. 192: 1019-1022. 
Axler, R. P., and J. E. Reuter. 1996. Nitrate uptake by phytoplankton and periphyton: Wholelake enrichments and mesocosm- ${ }^{15} \mathrm{~N}$ experiments in an oligotrophic lake. Limnol. Oceanogr. 41: 659-671.

Baer, K. N., and C. E. Goulden. 1998. Evaluation of a high-hardness COMBO medium and frozen algae for Daphnia magna. Ecotoxicol. Environ. Saf. 39: 201-206.

Ballantyne, A. P., M. T. Brett, and D. E. Schindler. 2003. The importance of dietary phosphorus and highly unsaturated fatty acids for sockeye (Oncorhynchus nerka) growth in Lake Washington a bioenergetics approach. Can. J. Fish. Aquat. Sci. 60: 12-22.

Barbiero, R. P. 2000. A multi-lake comparison of epilithic diatom communities on natural and artificial substrates. Hydrobiologia 438: 157-170.

Barranguet, C., S. A. M. van Beusekom, B. Veuger, T. R. Neu, E. M. M. Manders, J. J. Sinke, and W. Admiraal. 2004. Studying undisturbed autotrophic biofilms: still a technical challenge. Aquat. Microb. Ecol. 34: 1-9.

Bartrons, M., J. Catalan, and E. O. Casamayor. 2012. High bacterial diversity in epilithic biofilms of oligotrophic mountain lakes. Microb. Ecol. 64: 860-869.

Bastawrous, M., A. Jenne, M. Tabatabaei Anaraki, and A. J. Simpson. 2018. In-Vivo NMR Spectroscopy: A Powerful and Complimentary Tool for Understanding Environmental Toxicity. Metabolites 8: 35.

Baty, A. M., B. Frølund, G. G. Geesey, S. Langille, E. J. Quintero, P. A. Suci, and R. M. Weiner. 1996. Adhesion of biofilms to inert surfaces: a molecular level approach directed at the marine environment. Biofouling 10: 111-121.

Baulch, H. M., T. W. Nord, M. Y. Ackerman, J. D. Dale, R. R. O. Hazewinkel, D. W. Schindler, and R. D. Vinebrooke. 2003. Climate warming experiments: Design of a mesocosm heating system. Limnol. Oceanogr. Methods 1: 10-15.

Becker, C., and M. Boersma. 2003. Resource quality effects on life histories of Daphnia. Limnol. Oceanogr. 48: 700-706.

Becker, C., and M. Boersma. 2005. Differential effects of phosphorus and fatty acids on Daphnia magna growth and reproduction. Limnol. Oceanogr. 50: 388-397.

de Beer, D., P. Stoodley, and Z. Lewandowski. 1994. Liquid flow in heterogeneous biofilms. Biotechnol. Bioeng. 44: 636-641.

de Beer, D., P. Stoodley, and Z. Lewandowski. 1996. Liquid flow and mass transport in heterogeneous biofilms. Water Res. 30: 2761-2765.

Behning, A. L. 1924. On the study of bottom life of the Volga river. Monogr Volzhsk Biol Stants Saratov Ob Estestvoispyt 1: 1-398.

Behning, A. L. 1928. Das Leben der Wolga, Volume IV. Schweizerbart.

Bell, J. G., C. Ghioni, and J. R. Sargent. 1994. Fatty acid compositions of 10 freshwater invertebrates which are natural food organisms of Atlantic salmon parr (Salmo salar): a comparison with commercial diets. Aquaculture 128: 301-313.

Bell, M. V, J. R. Dick, T. R. Anderson, and D. W. Pond. 2007. Application of liposome and stable isotope tracer techniques to study polyunsaturated fatty acid biosynthesis in marine zooplankton. J. Plankton Res. 29: 417-422.

Bell, M. V, R. J. Henderson, and J. R. Sargent. 1986. The role of polyunsaturated fatty acids in fish. Comp. Biochem. Physiol. Part B Comp. Biochem. 83: 711-719.

Berlanga, M., and R. Guerrero. 2016. Living together in biofilms: the microbial cell factory and its biotechnological implications. Microb. Cell Fact. 15: 165. 
Bermudez, R., Y. Feng, M. Y. Roleda, and others. 2015. Long-term conditioning to elevated $\mathrm{pCO}_{2}$ and warming influences the fatty and amino acid composition of the diatom Cylindrotheca fusiformis. PLoS One 10: e0123945.

Besser, J. M., W. G. Brumbaugh, T. W. May, S. E. Church, and B. A. Kimball. 2001. Bioavailability of metals in stream food webs and hazards to brook trout (Salvelinus fontinalis) in the upper Animas River watershed, Colorado. Arch. Environ. Contam. Toxicol. 40: 48-59.

Bi, R., S. M. H. Ismar, U. Sommer, and M. Zhao. 2018. Simultaneous shifts in elemental stoichiometry and fatty acids of Emiliania huxleyi in response to environmental changes. Biogeosciences 15: 1029.

Bi, R., S. Ismar, U. Sommer, and M. Zhao. 2017. Environmental dependence of the correlations between stoichiometric and fatty acid-based indicators of phytoplankton nutritional quality. Limnol. Oceanogr. 62: 334-347.

Biggs, B. J. F., and M. E. Close. 1989. Periphyton biomass dynamics in gravel bed rivers: the relative effects of flows and nutrients. Freshw. Biol. 22: 209-231.

Björk-Ramberg, S. 1985. Uptake of phosphate and inorganic nitrogen by a sediment-algal system in a subarctic lake. Freshw. Biol. 15: 175-183.

Bloesch, J., P. Bossard, H. Bührer, H. R. Bürgi, and U. Uehlinger. 1988. Can results from limnocorral experiments be transferred to in situ conditions? Hydrobiologia 159: 297-308.

Blumenshine, S. C., Y. Vadeboncoeur, D. M. Lodge, K. L. Cottingham, and S. E. Knight. 1997. Benthic-pelagic links: responses of benthos to water-column nutrient enrichment. J. North Am. Benthol. Soc. 16: 466-479.

Bly, J. E., T. M. Buttke, and L. W. Clem. 1990. Differential effects of temperature and exogenous fatty acids on mitogen-induced proliferation in channel catfish $\mathrm{T}$ and $\mathrm{B}$ lymphocytes. Comp. Biochem. Physiol. Part A Physiol. 95: 417-424.

Boersma, M., C. Schöps, and E. McCauley. 2001. Nutritional quality of seston for the freshwater herbivore Daphnia galeata $\times$ hyalina: biochemical versus mineral limitations. Oecologia 129: 342-348.

Böhme, A., U. Risse-Buhl, and K. Küsel. 2009. Protists with different feeding modes change biofilm morphology. FEMS Microbiol. Ecol. 69: 158-169.

Booth, S. C., M. L. Workentine, J. Wen, R. Shaykhutdinov, H. J. Vogel, H. Ceri, R. J. Turner, and A. M. Weljie. 2011. Differences in metabolism between the biofilm and planktonic response to metal stress. J. Proteome Res. 10: 3190-3199.

Bootsma, H. A., R. E. Hecky, R. H. Hesslein, and G. F. Turner. 1996. Food partitioning among Lake Malawi nearshore fishes as revealed by stable isotope analyses. Ecology 77: 12861290.

Borisova, E. V, O. N. Makhutova, M. I. Gladyshev, and N. N. Sushchik. 2016. Fluxes of biomass and essential polyunsaturated fatty acids from water to land via chironomid emergence from a mountain lake. Contemp. Probl. Ecol. 9: 446-457.

Bos, R., H. C. van der Mei, and H. J. Busscher. 1999. Physico-chemistry of initial microbial adhesive interactions-its mechanisms and methods for study. FEMS Microbiol. Rev. 23: 179-230.

Boston, H. L., and W. R. Hill. 1991. Photosynthesis-light relations of stream periphyton communities. Limnol. Oceanogr. 36: 644-656. 
Bothwell, M. L. 1985. Phosphorus limitation of lotic periphyton growth rates: An intersite comparison using continuous-flow troughs (Thompson River system, British Columbia). Limnol. Oceanogr. 30: 527-542.

Bothwell, M. L. 1988. Growth rate responses of lotic periphytic diatoms to experimental phosphorus enrichment: the influence of temperature and light. Can. J. Fish. Aquat. Sci. 45: 261-270.

Bothwell, M. L. 1989. Phosphorus-limited growth dynamics of lotic periphytic diatom communities: areal biomass and cellular growth rate responses. Can. J. Fish. Aquat. Sci. 46: 1293-1301.

Bothwell, M. L., D. Sherbot, and C. M. Pollock. 1994. Ecosystem response to solar ultraviolet-B radiation: influence of trophic-level interactions. Science. 265: 97-100.

Bothwell, M. L., D. Sherbot, A. C. Roberge, and R. J. Daley. 1993. Influence of natural ultraviolet radiation on lotic periphytic diatom community growth, biomass accrual, and species composition: Short-term versus long-term effects. J. Phycol. 29: 24-35.

Bott, T. L. 1975. Bacterial growth rates and temperature optima in a stream with a fluctuating thermal regime. Limnol. Oceanogr. 20: 191-197.

Bourassa, N., and A. Cattaneo. 2000. Responses of a lake outlet community to light and nutrient manipulation: effects on periphyton and invertebrate biomass and composition. Freshw. Biol. 44: 629-639.

Bowden, W. B. 1987. The biogeochemistry of nitrogen in freshwater wetlands. Biogeochemistry 4: 313-348.

Box, G. E. P., and D. R. Cox. 1964. An analysis of transformations. J. R. Stat. Soc. Ser. B 211252.

Box, G. E. P., and D. R. Cox. 1981. An analysis of transformations revisited, rebutted. Wisconsin Univ-Madison Mathematics Research Center.

Bradshaw, D. J., P. D. Marsh, R. J. Hodgson, and J. M. Visser. 2002. Effects of glucose and fluoride on competition and metabolism within in vitro dental bacterial communities and biofilms. Caries Res. 36: 81-86.

Brauge, C., G. Corraze, and F. Médale. 1995. Effects of dietary levels of carbohydrate and lipid on glucose oxidation and lipogenesis from glucose in rainbow trout, Oncorhynchus mykiss, reared in freshwater or in seawater. Comp. Biochem. Physiol. Part A Physiol. 111: 117124.

Brenner, R. R. 1974. The oxidative desaturation of unsaturated fatty acids in animals. Mol. Eellular Biochem. 3: 41-52.

Brett, M. T., M. J. Kainz, S. J. Taipale, and H. Seshan. 2009. Phytoplankton, not allochthonous carbon, sustains herbivorous zooplankton production. Proc. Natl. Acad. Sci. 106: $21197-$ 21201.

Brett, M. T., and D. C. Müller-Navarra. 1997. The role of highly unsaturated fatty acids in aquatic foodweb processes. Freshw. Biol. 38: 483-499.

Brett, M. T., D. C. Müller-Navarra, A. P. Ballantyne, J. L. Ravet, and C. R. Goldman. 2006. Daphnia fatty acid composition reflects that of their diet. Limnol. Oceanogr. 51: 24282437.

Brock, T. D. 1967. Relationship between standing crop and primary productivity along a hot spring thermal gradient. Ecology 48: 566-571.

Bronmark, C., S. P. Klosiewski, and R. A. Stein. 1992. Indirect effects of predation in a freshwater, benthic food chain. Ecology 73: 1662-1674. 
Brooks, S., G. T. Clark, S. M. Wright, R. J. Trueman, A. D. Postle, A. R. Cossins, and N. Maclean. 2002. Electrospray ionisation mass spectrometric analysis of lipid restructuring in the carp (Cyprinus carpio L.) during cold acclimation. J. Exp. Biol. 205: 3989-3997.

Brown, H. B. 1908. Algal periodicity in certain ponds and streams. Bull. Torrey Bot. Club 35: 223-248.

Buckner, J. S., and M. M. Hagen. 2003. Triacylglycerol and phospholipid fatty acids of the silverleaf whitefly: composition and biosynthesis. Arch. Insect Biochem. Physiol. 53: 6679.

Budge, S. M., and C. C. Parrish. 1998. Lipid biogeochemistry of plankton, settling matter and sediments in Trinity Bay, Newfoundland. II. Fatty acids. Org. Geochem. 29: 1547-1559.

Bunn, S. E., and P. I. Boon. 1993. What sources of organic carbon drive food webs in billabongs? A study based on stable isotope analysis. Oecologia 96: 85-94.

Bunn, S. E., P. M. Davies, and M. Winning. 2003. Sources of organic carbon supporting the food web of an arid zone floodplain river. Freshw. Biol. 48: 619-635.

Burkholder, J. M., R. G. Wetzel, and K. L. Klomparens. 1990. Direct comparison of phosphate uptake by adnate and loosely attached microalgae within an intact biofilm matrix. Appl. Environ. Microbiol. 56: 2882-2890.

Burnett, D. A., P. D. Champion, J. S. Clayton, and J. Ogden. 2007. A system for investigation of the temperature responses of emergent aquatic plants. Aquat. Bot. 86: 187-190.

Burns, C. W., M. T. Brett, and M. Schallenberg. 2011. A comparison of the trophic transfer of fatty acids in freshwater plankton by cladocerans and calanoid copepods. Freshw. Biol. 56: 889-903.

Burton, T. M., M. P. Oemke, and J. M. Molloy. 1991. Contrasting effects of nitrogen and phosphorus additions on epilithic algae in a hardwater and a softwater stream in northern Michigan. Int. Vereinigung für Theor. und Angew. Limnol. Verhandlungen 24: 1644-1653.

Butterwick, C., S. I. Heaney, and J. F. Talling. 2005. Diversity in the influence of temperature on the growth rates of freshwater algae, and its ecological relevance. Freshw. Biol. 50: 291300.

Cadmus, P., J. P. F. Pomeranz, and J. M. Kraus. 2016. Low-cost floating emergence net and bottle trap: comparison of two designs. J. Freshw. Ecol. 31: 653-658.

Campbell, L. M., D. W. Schindler, D. C. G. Muir, D. B. Donald, and K. A. Kidd. 2000. Organochlorine transfer in the food web of subalpine Bow Lake, Banff National Park. Can. J. Fish. Aquat. Sci. 57: 1258-1269.

Camps, M., A. Barani, G. Gregori, A. Bouchez, B. Le Berre, C. Bressy, Y. Blache, and J. F. Briand. 2014. Antifouling coatings influence both abundance and community structure of colonizing biofilms: a case study in the Northwestern Mediterranean sea. Appl. Environ. Microbiol. 80: 4821-4831.

Canale, R. P., and A. H. Vogel. 1974. Effects of temperature on phytoplankton growth. J. Environ. Eng. Div. 100: 231-241.

Cao, Y., W. Li, and E. Jeppesen. 2014. The response of two submerged macrophytes and periphyton to elevated temperatures in the presence and absence of snails: a microcosm approach. Hydrobiologia 738: 49-59.

Cao, Y., S. Olsen, M. F. Gutierrez, and others. 2017. Temperature effects on periphyton, epiphyton and epipelon under a nitrogen pulse in low-nutrient experimental freshwater lakes. Hydrobiologia 795: 267-279. 
Cargill, A. S., K. W. Cummins, B. J. Hanson, and R. R. Lowry. 1985. The role of lipids, fungi, and temperature in the nutrition of a shredder caddisfly, Clistoronia magnifica. Freshw. Invertebr. Biol. 4: 64-78.

Carlton, R. G., and R. G. Wetzel. 1987. Distributions and fates of oxygen in periphyton communities. Can. J. Bot. 65: 1031-1037.

Carlton, R. G., and R. G. Wetzel. 1988. Phosphorus flux from lake sediments: effect of epipelic algal oxygen production. Limnol. Oceanogr. 33: 562-570.

Carpenter, S. R. 1999. Microcosm experiments have limited relevance for community and ecosystem ecology: reply. Ecology 80: 1085-1088.

Carr, G. M., A. Morin, and P. A. Chambers. 2005. Bacteria and algae in stream periphyton along a nutrient gradient. Freshw. Biol. 50: 1337-1350.

Cashman, M. J., J. D. Wehr, and K. Truhn. 2013. Elevated light and nutrients alter the nutritional quality of stream periphyton. Freshw. Biol. 58: 1447-1457.

Castenholz, R. W. 1960. Seasonal changes in the attached algae of freshwater and saline lakes in the Lower Grand Coulee, Washington. Limnol. Oceanogr. 5: 1-28.

Cattaneo, A., and M. C. Amireault. 1992. How artificial are artificial substrata for periphyton? J. North Am. Benthol. Soc. 11: 244-256.

IUPAC-IUB Commission on Biochemical Nomenclature (CBN). 1977. The Nomenclature of Lipids: recommendations, 1976. Eur. J. Biochem. 79: 11-21.

Chao, J., G. M. Wolfaardt, and M. T. Arts. 2010. Characterization of Pseudomonas aeruginosa fatty acid profiles in biofilms and batch planktonic cultures. Can. J. Microbiol. 56: 10281039.

Characklis, W. G., and K. E. Cooksey. 1983. Biofilms and microbial fouling. Adv. Appl. Microbiol. 29: 93-138.

Chen, C. C., J. E. Petersen, and W. M. Kemp. 1997. Spatial and temporal scaling of periphyton growth on walls of estuarine mesocosms. Mar. Ecol. Prog. Ser. 155: 1-15.

Chi, X., Q. Yang, F. Zhao, S. Qin, Y. Yang, J. Shen, and H. Lin. 2008. Comparative analysis of fatty acid desaturases in cyanobacterial genomes. Comp. Funct. Genomics. 284508.

Choi, O., C. P. Yu, G. E. Fernández, and Z. Hu. 2010. Interactions of nanosilver with Escherichia coli cells in planktonic and biofilm cultures. Water Res. 44: 6095-6103.

Christensen, M. R., M. D. Graham, R. D. Vinebrooke, D. L. Findlay, M. J. Paterson, and M. A. Turner. 2006. Multiple anthropogenic stressors cause ecological surprises in boreal lakes. Glob. Chang. Biol. 12: 2316-2322.

Ciais, P., C. Sabine, G. Bala, and others. 2014. Carbon and other biogeochemical cycles, p. 465570. In T. F. Stocker, D. Qin, G. K. Plattner, M. Tignor, S. K. Allen, J. Boschung, A. Nauels, Y. Xia, V. Bex and P. M. Midgley [eds.], Climate change 2013: The physical science basis. Contribution of working group I to the fifth assessment report of the Intergovernmental Panel on Climate Change. Cambridge University Press.

Clarke, A., and N. M. Johnston. 1999. Scaling of metabolic rate with body mass and temperature in teleost fish. J. Anim. Ecol. 68: 893-905.

Coats, R., J. Perez-Losada, G. Schladow, R. Richards, and C. R. Goldman. 2006. The warming of lake Tahoe. Clim. Change 76: 121-148.

Cockell, C. S., P. Rettberg, E. Rabbow, and K. Olsson-Francis. 2011. Exposure of phototrophs to 548 days in low Earth orbit: microbial selection pressures in outer space and on early earth. ISME J. 5: 1671. 
Coles, J. F., and R. C. Jones. 2000. Effect of temperature on photosynthesis-light response and growth of four phytoplankton species isolated from a tidal freshwater river. J. Phycol. 36: 7-16.

Colletti, P. J., D. W. Blinn, A. Pickart, and V. T. Wagner. 1987. Influence of different densities of the mayfly grazer Heptagenia criddlei on lotic diatom communities. J. North Am. Benthol. Soc. 6: 270-280.

Collins, M., R. Knutti, J. Arblaster, and others. 2013. Long-term climate change: projections, commitments and irreversibility. In T. F. Stocker, D. Qin, G. K. Plattner, M. Tignor, S. K. Allen, J. Boschung, A. Nauels, Y. Xia, V. Bex and P. M. Midgley [eds.], Climate change 2013: The physical science basis. Contribution of working group I to the fifth assessment report of the Intergovernmental Panel on Climate Change. Cambridge University Press.

Colombo, S. M., A. Wacker, C. C. Parrish, M. J. Kainz, and M. T. Arts. 2016. A fundamental dichotomy in long-chain polyunsaturated fatty acid abundance between and within marine and terrestrial ecosystems. Environ. Rev. 25: 163-174.

Compere, C., M. Bellon-Fontaine, P. Bertrand, and others. 2001. Kinetics of conditioning layer formation on stainless steel immersed in seawater. Biofouling 17: 129-145.

Congestri, R., F. Di Pippo, R. De Philippis, I. Buttino, G. Paradossi, and P. Albertano. 2006. Seasonal succession of phototrophic biofilms in an Italian wastewater treatment plant: biovolume, spatial structure and exopolysaccharides. Aquat. Microb. Ecol. 45: 301-312.

Conklin, D. E., and L. Provasoli. 1977. Nutritional requirements of the water flea Moina macrocopa. Biol. Bull. 152: 337-350.

Cook, J., D. Nuccitelli, S. A. Green, and others. 2013. Quantifying the consensus on anthropogenic global warming in the scientific literature. Environ. Res. Lett. 8: 24024.

Cook, J., N. Oreskes, P. T. Doran, and others. 2016. Consensus on consensus: A synthesis of consensus estimates on human-caused global warming. Environ. Res. Lett. 11: 1-7.

Cook, P. L. M., B. Veuger, S. Böer, and J. J. Middelburg. 2007. Effect of nutrient availability on carbon and nitrogen incorporation and flows through benthic algae and bacteria in nearshore sandy sediment. Aquat. Microb. Ecol. 49: 165-180.

Cooke, W. B. 1956. Colonization of artificial bare areas by microorganisms. Bot. Rev. 22: 613638.

Cooksey, K. E. 1992. Extracellular polymers in biofilms, p. 137-147. In L.F. Melo, T.R. Bott, M. Fletcher and B. Capdeville [eds.], Biofilms — science and technology. Springer.

Cossins, A. R., P. A. Murray, A. Y. Gracey, and others. 2002. The role of desaturases in coldinduced lipid restructuring. Biochem. Soc. Trans. 30: 1082-1086.

Coveney, M. F., and R. G. Wetzel. 1989. Bacterial metabolism of algal extracellular carbon. Hydrobiologia 173: 141-149.

Cox, E. J. 1990. Studies on the algae of a small softwater stream I. Occurence and distribution with particular reference to the diatoms. Arch. für Hydrobiol. (Monographische Beiträge) 83: 525-552.

Cox, E. J. 1993. Freshwater diatom ecology: developing an experimental approach as an aid to interpreting field data. Twelfth International Diatom Symposium. Springer. 447-452.

Crockett, E. L. 1998. Cholesterol function in plasma membranes from ectotherms: membranespecific roles in adaptation to temperature. Am. Zool. 38: 291-304.

Cui, Y., L. Jin, S. R. Ko, and others. 2017. Periphyton effects on bacterial assemblages and harmful cyanobacterial blooms in a eutrophic freshwater lake: a mesocosm study. Sci. Rep. 7: 7827. 
Cunnane, S. C. 2003. Problems with essential fatty acids: time for a new paradigm? Prog. Lipid Res. 42: 544-568.

Dadd, R. H., J. E. Kleinjan, and D. W. Stanley-Samuelson. 1987. Polyunsaturated fatty acids of mosquitos reared with single dietary polyunsaturates. Insect Biochem. 17: 7-16.

Dahlgren, K., A. K. E. Wiklund, and A. Andersson. 2011. The influence of autotrophy, heterotrophy and temperature on pelagic food web efficiency in a brackish water system. Aquat. Ecol. 45: 307-323.

Dalsgaard, J., M. S. John, G. Kattner, D. C. Müller-Navarra, and W. Hagen. 2003. Fatty acid trophic markers in the pelagic marine environment, Adv. Mar. Biol. 46: 225-340.

Dang, H., and C. R. Lovell. 2000. Bacterial primary colonization and early succession on surfaces in marine waters as determined by amplified rRNA gene restriction analysis and sequence analysis of 16S rRNA genes. Appl. Environ. Microbiol. 66: 467-475.

Dang, H., and C. R. Lovell. 2016. Microbial surface colonization and biofilm development in marine environments. Microbiol. Mol. Biol. Rev. 80: 91-138.

Danielsdottir, M. G., M. T. Brett, and G. B. Arhonditsis. 2007. Phytoplankton food quality control of planktonic food web processes. Hydrobiologia 589: 29-41.

Daufresne, M., K. Lengfellner, and U. Sommer. 2009. Global warming benefits the small in aquatic ecosystems. Proc. Natl. Acad. Sci. 106: 12788-12793.

Dauta, A., J. Devaux, F. Piquemal, and L. Boumnich. 1990. Growth rate of four freshwater algae in relation to light and temperature. Hydrobiologia 207: 221-226.

Davies-Colley, R. J., C. W. Hickey, J. M. Quinn, and P. A. Ryan. 1992. Effects of clay discharges on streams. Hydrobiologia 248: 215-234.

Davies-Colley, R. J., and J. M. Quinn. 1998. Stream lighting in five regions of North Island, New Zealand: control by channel size and riparian vegetation. New Zeal. J. Mar. Freshw. Res. 32: 591-605.

Davies-Colley, R. J., and J. W. Nagels. 2008. Predicting light penetration into river waters. J. Geophys. Res. Biogeosciences 113: G03028.

Davison, I. R. 1991. Environmental effects on algal photosynthesis: temperature. J. Phycol. 27: $2-8$.

Decho, A. W. 1990. Microbial exopolymer secretions in ocean environments: their role(s) in food webs and marine processes. Ocean. Mar. Biol. Annu. Rev 28: 73-153.

Demott, W., and D. C. Müller-Navarra. 1997. The importance of highly unsaturated fatty acids in zooplankton nutrition: evidence from experiments with Daphnia, a cyanobacterium and lipid emulsions. Freshw. Biol. 38: 649-664.

DeNicola, D. M. 1996. Periphyton responses to temperature at different ecological levels, p. 149-181. In R. J. Stevenson, M. L. Bothwell, and R. L. Lowe [eds.], Aquatic ecology. Academic Press.

DeNicola, D. M., K. D. Hoagland, and S. C. Roemer. 1992. Influences of canopy cover on spectral irradiance and periphyton assemblages in a prairie stream. J. North Am. Benthol. Soc. 11: 391-404.

DeNicola, D. M., and C. D. McIntire. 1991. Effects of hydraulic refuge and irradiance on grazerperiphyton interactions in laboratory streams. J. North Am. Benthol. Soc. 10: 251-262.

Denoux, C., D. Martin-Creuzburg, A.M. Koussoroplis, F. Perriere, C. Desvilettes, G. Bourdier, and A. Bec. 2017. Phospholipid-bound eicosapentaenoic acid (EPA) supports higher fecundity than free EPA in Daphnia magna. J. Plankton Res. 39: 843-848. 
Derlon, N., N. Koch, B. Eugster, T. Posch, J. Pernthaler, W. Pronk, and E. Morgenroth. 2013. Activity of metazoa governs biofilm structure formation and enhances permeate flux during Gravity-Driven Membrane (GDM) filtration. Water Res. 47: 2085-2095.

Derlon, N., M. Peter-Varbanets, A. Scheidegger, W. Pronk, and E. Morgenroth. 2012. Predation influences the structure of biofilm developed on ultrafiltration membranes. Water Res. 46: 3323-3333.

Descy, J. P., and C. Mouvet. 1984. Impact of the Tihange nuclear power plant on the periphyton and the phytoplankton of the Meuse River (Belgium). Hydrobiologia 119: 119-128.

Desvilettes, C., G. Bourdier, and J. C. Breton. 1997a. On the occurrence of a possible bioconversion of linolenic acid into docosahexaenoic acid by the copepod Eucyclops serrulatus fed on microalgae. J. Plankton Res. 19: 273-278.

Desvilettes, C. H., G. Bourdier, C. H. Amblard, and B. Barth. 1997b. Use of fatty acids for the assessment of zooplankton grazing on bacteria, protozoans and microalgae. Freshw. Biol. 38: 629-637.

van Dijk, G. M. 1993. Dynamics and attenuation characteristics of periphyton upon artificial substratum under various light conditions and some additional observations on periphyton upon Potamogeton pectinatus L. Hydrobiologia 252: 143-161.

Dodds, W. K. 1992. A modified fiber-optic light microprobe to measure spherically integrated photosynthetic photon flux density: Characterization of periphyton photosynthesisirradiance patterns. Limnol. Oceanogr. 37: 871-878.

Dodds, W. K. 2003. The role of periphyton in phosphorus retention in shallow freshwater aquatic systems. J. Phycol. 39: 840-849.

Dodds, W. K., B. J. F. Biggs, and R. L. Lowe. 1999. Photosynthesis-Irradiance patterns in benthic microalgae: variations as a function of assemblage thickness and community structure. J. Phycol. 35: 42-53.

Donlan, R. M. 2002. Biofilms: microbial life on surfaces. Emerg. Infect. Dis. 8: 881.

Dopheide, A., G. Lear, R. Stott, and G. Lewis. 2011. Preferential feeding by the ciliates Chilodonella and Tetrahymena spp. and effects of these protozoa on bacterial biofilm structure and composition. Appl. Environ. Microbiol. 77: 4564-4572.

Downing, J. A., Y. T. Prairie, J. J. Cole, and others. 2006. The global abundance and size distribution of lakes, ponds, and impoundments. Limnol. Oceanogr. 51: 2388-2397.

Downing, J. A., S. B. Watson, and E. McCauley. 2001. Predicting cyanobacteria dominance in lakes. Can. J. Fish. Aquat. Sci. 58: 1905-1908.

Dring, M. J. 1981. Chromatic adaptation of photosynthesis in benthic marine algae: an examination of its ecological significance using a theoretical model. Limnol. Oceanogr. 26: 271-284.

Dubois-Brissonnet, F., E. Trotier, and R. Briandet. 2016. The biofilm lifestyle involves an increase in bacterial membrane saturated fatty acids. Front. Microbiol. 7: 1673.

Dudgeon, D., A. H. Arthington, M. O. Gessner, and others. 2006. Freshwater biodiversity: importance, threats, status and conservation challenges. Biol. Rev. 81: 163-182.

Duncan, S. W., and D. W. Blinn. 1989. Importance of physical variables on the seasonal dynamics of epilithic algae in a highly shaded canyon stream. J. Phycol. 25: 455-461.

Dunstan, G. A., J. K. Volkman, S. M. Barrett, J.M. Leroi, and S. W. Jeffrey. 1993. Essential polyunsaturated fatty acids from 14 species of diatom (Bacillariophyceae). Phytochemistry 35: $155-161$. 
Dzialowski, A. R., M. Rzepecki, I. Kostrzewska-Szlakowska, K. Kalinowska, A. Palash, and J. T. Lennon. 2014. Are the abiotic and biotic characteristics of aquatic mesocosms representative of in situ conditions? J. Limnol. 73: 603-612.

Ehlers, G. A. C., and S. Turner. 2012. Biofilms in wastewater treatment systems, p. 99-110. In G. Lear and G. D. Lewis [eds.], Microbial biofilms: current research and applications. Caister Academic Press.

Eichenberger, E., and A. Schlatter. 1978. Effect of herbivorous insects on the production of benthic algal vegetation in outdoor channels. Int. Vereinigung für Theor. und Angew. Limnol. Verhandlungen 20: 1806-1810.

Ekin, I., M. Başhan, and R. Şeşen. 2011. Possible seasonal variation of the fatty acid composition from Melanopsis praemorsa (L., 1758) (Gastropoda: Prosobranchia), from southeast Anatolia, Turkey. Turkish J. Biol. 35: 203-213.

von Elert, E. 2002. Determination of limiting polyunsaturated fatty acids in Daphnia galeata using a new method to enrich food algae with single fatty acids. Limnol. Oceanogr. 47: 1764-1773.

von Elert, E. 2004. Food quality constraints in Daphnia: interspecific differences in the response to the absence of a long chain polyunsaturated fatty acid in the food source. Hydrobiologia 526: $187-196$.

Ellen, C. 2017. The effects of water temperature on fatty acid content in the diatom, Navicula pelliculosa. MSc Thesis, Ryerson University, Toronto, Ontario.

Elliott, J. A. 2012. Is the future blue-green? A review of the current model predictions of how climate change could affect pelagic freshwater cyanobacteria. Water Res. 46: 1364-1371.

Eloranta, P. V. 1982. Periphyton growth and diatom community structure in a cooling water pond. Hydrobiologia 96: 253-265.

Elser, J. J., M. E. S. Bracken, E. E. Cleland, and others. 2007. Global analysis of nitrogen and phosphorus limitation of primary producers in freshwater, marine and terrestrial ecosystems. Ecol. Lett. 10: 1135-1142.

Elser, J. J., E. R. Marzolf, and C. R. Goldman. 1990. Phosphorus and nitrogen limitation of phytoplankton growth in the freshwaters of North America: a review and critique of experimental enrichments. Can. J. Fish. Aquat. Sci. 47: 1468-1477.

Elwood, J. W., J. D. Newbold, A. F. Trimble, and R. W. Stark. 1981. The limiting role of phosphorus in a woodland stream ecosystem: effects of $\mathrm{P}$ enrichment on leaf decomposition and primary producers. Ecology 62: 146-158.

Emtiazi, F., T. Schwartz, S. M. Marten, P. Krolla-Sidenstein, and U. Obst. 2004. Investigation of natural biofilms formed during the production of drinking water from surface water embankment filtration. Water Res. 38: 1197-1206.

Eppley, R. W. 1972. Temperature and phytoplankton growth in the sea. Fish. Bull. Natl. Ocean. Atmos. Adm. 70: 1063-1085.

Ernst, R., C. S. Ejsing, and B. Antonny. 2016. Homeoviscous adaptation and the regulation of membrane lipids. J. Mol. Biol. 428: 4776-4791.

Espeland, E. M., S. N. Francoeur, and R. G. Wetzel. 2001. Influence of algal photosynthesis on biofilm bacterial production and associated glucosidase and xylosidase activities. Microb. Ecol. 42: 524-530.

Evans, D., and J. G. Stockner. 1972. Attached algae on artificial and natural substrates in Lake Winnipeg, Manitoba. J. Fish. Board Canada 29: 31-44. 
Fairchild, G. W., and A. C. Everett. 1988. Effects of nutrient (N, P, C) enrichment upon periphyton standing crop, species composition and primary production in an oligotrophic softwater lake. Freshw. Biol. 19: 57-70.

Fairchild, G. W., R. L. Lowe, and W. B. Richardson. 1985. Algal periphyton growth on nutrientdiffusing substrates: an in situ bioassay. Ecology 66: 465-472.

Fairchild, G. W., and J. W. Sherman. 1993. Algal periphyton response to acidity and nutrients in softwater lakes: lake comparison vs. nutrient enrichment approaches. J. North Am. Benthol. Soc. 12: $157-167$.

Fairchild, G. W., J. W. Sherman, and F. W. Acker. 1989. Effects of nutrient (N, P, C) enrichment, grazing and depth upon littoral periphyton of a softwater lake. Hydrobiologia 173: $69-83$.

Fanta, S. E., W. R. Hill, T. B. Smith, and B. J. Roberts. 2010. Applying the light: nutrient hypothesis to stream periphyton. Freshw. Biol. 55: 931-940.

Farag, A. M., D. Skaar, D. A. Nimick, E. MacConnell, and C. Hogstrand. 2003. Characterizing aquatic health using salmonid mortality, physiology, and biomass estimates in streams with elevated concentrations of arsenic, cadmium, copper, lead, and zinc in the Boulder River watershed, Montana. Trans. Am. Fish. Soc. 132: 450-467.

Farkas, T., G. Y. Nemecz, and I. Csengeri. 1984. Differential response of lipid metabolism and membrane physical state by an actively and passively overwintering planktonic crustacean. Lipids 19: 436-442.

Fauré-Fremiet, E. 1951. The tidal rhythm of the diatom Hantzschia amphioxys. Biol. Bull. 100: $173-177$.

Favre, L., A. Ortalo-Magné, C. Pichereaux, A. Gargaros, O. Burlet-Schiltz, V. Cotelle, and G. Culioli. 2018. Metabolome and proteome changes between biofilm and planktonic phenotypes of the marine bacterium Pseudoalteromonas lipolytica TC8. Biofouling 34: $132-148$.

Federer, C. A., and C. B. Tanner. 1966. Spectral distribution of light in the forest. Ecology 47: 555-560.

Feminella, J. W., and C. P. Hawkins. 1995. Interactions between stream herbivores and periphyton: a quantitative analysis of past experiments. J. North Am. Benthol. Soc. 14: 465509.

Feminella, J. W., M. E. Power, and V. H. Resh. 1989. Periphyton responses to invertebrate grazing and riparian canopy in three northern California coastal streams. Freshw. Biol. 22: 445-457.

Fereidouni, A. E., N. Fathi, and M. K. Khalesi. 2013. Enrichment of Daphnia magna with canola oil and its effects on the growth, survival and stress resistance of the Caspian kutum (Rutilus frisii kutum) larvae. Turkish J. Fish. Aquat. Sci. 13: 119-126.

Ferragut, C., A. F. Rodello, and C. E. de M. Bicudo. 2010. Seasonal variability of periphyton nutrient status and biomass on artificial and natural substrates in a tropical mesotrophic reservoir. Acta Limnol. Bras. 22: 397-409.

Feuchtmayr, H., D. McKee, I. Harvey, D. Atkinson, and B. Moss. 2007. Response of macroinvertebrates to warming, nutrient addition and predation in large-scale mesocosm tanks. Hydrobiologia 584: 425-432. 
Feuchtmayr, H., R. Moran, K. Hatton, L. Connor, T. Heyes, B. Moss, I. Harvey, and D. Atkinson. 2009. Global warming and eutrophication: effects on water chemistry and autotrophic communities in experimental hypertrophic shallow lake mesocosms. J. Appl. Ecol. 46: 713-723.

Feuchtmayr, H., B. Moss, I. Harvey, R. Moran, K. Hatton, L. Connor, and D. Atkinson. 2010. Differential effects of warming and nutrient loading on the timing and size of the spring zooplankton peak: an experimental approach with hypertrophic freshwater mesocosms. J. Plankton Res. 32: 1715-1725.

Fields, M. W., A. Hise, E. J. Lohman, and others. 2014. Sources and resources: importance of nutrients, resource allocation, and ecology in microalgal cultivation for lipid accumulation. Appl. Microbiol. Biotechnol. 98: 4805-4816.

Fietz, S., G. Kobanova, L. Izmest'eva, and A. Nicklisch. 2005. Regional, vertical and seasonal distribution of phytoplankton and photosynthetic pigments in Lake Baikal. J. Plankton Res. 27: 793-810.

Fischer, E. M., and R. Knutti. 2015. Anthropogenic contribution to global occurrence of heavyprecipitation and high-temperature extremes. Nat. Clim. Chang. 5: 560-564.

Flaim, G., U. Obertegger, A. Anesi, and G. Guella. 2014. Temperature-induced changes in lipid biomarkers and mycosporine-like amino acids in the psychrophilic dinoflagellate Peridinium aciculiferum. Freshw. Biol. 59: 985-997.

Flaim, G., U. Obertegger, and G. Guella. 2012. Changes in galactolipid composition of the cold freshwater dinoflagellate Borghiella dodgei in response to temperature, p. 285-293. In N. Salmaso, L. Naselli-Flores, L. Cerasino, G. Flaim, M. Tolotti, and J. Padisák [eds.], Phytoplankton responses to human impacts at different scales. Springer.

Flemming, H. C. 2008. Why microorganisms live in biofilms and the problem of biofouling, In Springer series on biofilms. Springer.

Flemming, H. C., and J. Wingender. 2010. The biofilm matrix. Nat. Rev. Microbiol. 8: 623.

Flemming, H. C., J. Wingender, U. Szewzyk, P. Steinberg, S. A. Rice, and S. Kjelleberg. 2016. Biofilms: an emergent form of bacterial life. Nat. Rev. Microbiol. 14: 563.

Fogg, G. E. 1969. Survival of algae under adverse conditions. Symposia of the society for experimental biology. 123-142.

Folch, J., M. Lees, and G. H. Sloane Stanley. 1957. A simple method for the isolation and purification of total lipids from animal tissues. J Biol Chem 226: 497-509.

Ford, R. C., and J. Barber. 1983. Incorporation of sterol into chloroplast thylakoid membranes and its effect on fluidity and function. Planta 158: 35-41.

France, R. L. 1995. Differentiation between littoral and pelagic food webs in lakes using stable carbon isotopes. Limnol. Oceanogr. 40: 1310-1313.

Friberg, N., J. Bergfur, J. Rasmussen, and L. Sandin. 2013. Changing Northern catchments: Is altered hydrology, temperature or both going to shape future stream communities and ecosystem processes? Hydrol. Process. 27: 734-740.

Fritsch, F. E. 1906. Problems in aquatic biology, with special reference to the study of algal periodicity. New Phytol. 5: 149-169.

Fritz, K. A., L. J. Kirschman, S. D. McCay, J. T. Trushenski, R. W. Warne, and M. R. Whiles. 2017. Subsidies of essential nutrients from aquatic environments correlate with immune function in terrestrial consumers. Freshw. Sci. 36: 893-900.

Fröls, S. 2013. Archaeal biofilms: widespread and complex. Biochem. Soc. Trans. 41: 393-398. 
Frost, P. C., and J. J. Elser. 2002. Effects of light and nutrients on the net accumulation and elemental composition of epilithon in boreal lakes. Freshw. Biol. 47: 173-183.

Früh, D., H. Norf, and M. Weitere. 2011. Response of biofilm-dwelling ciliate communities to enrichment with algae. Aquat. Microb. Ecol. 63: 299-309.

Fuschino, J. R., I. A. Guschina, G. Dobson, N. D. Yan, J. L. Harwood, and M. T. Arts. 2011. Rising water temperatures alter lipid dynamics and reduce n-3 essential fatty acid concentrations in Scenedesmus obliquus (Chlorophyta). J. Phycol. 47: 763-774.

Gallegos, C. L., and K. A. Moore. 2000. Factors contributing to water-column light attenuation, In SAV technical synthesis II.

Galloway, A. W. E., and M. Winder. 2015. Partitioning the relative importance of phylogeny and environmental conditions on phytoplankton fatty acids. PLoS One 10: e0130053.

Gao, X., O. A. Olapade, M. W. Kershner, and L. G. Leff. 2004. Algal-bacterial co-variation in streams: a cross-stream comparison. Arch. für Hydrobiol. 159: 253-261.

Garzke, J., T. Hansen, S. M. H. Ismar, and U. Sommer. 2016. Combined effects of ocean warming and acidification on copepod abundance, body size and fatty acid content. PLoS One 11: e0155952.

Gerten, D., and R. Adrian. 2001. Differences in the persistency of the North Atlantic Oscillation signal among lakes. Limnol. Oceanogr. 46: 448-455.

Ghioni, C., J. G. Bell, and J. R. Sargent. 1996. Polyunsaturated fatty acids in neutral lipids and phospholipids of some freshwater insects. Comp. Biochem. Physiol. Part B Biochem. Mol. Biol. 114: 161-170.

Gholami, M. 2010. Effects of n-3 HUFA enriched Daphnia magna on growth, survival, stress resistance and fatty acid composition of white fish fry (Rutilus frisii kutum). J. Fish. Aquat. Sci. 5: 49-55.

Gladyshev, M. I., M. T. Arts, and N. N. Sushchik. 2009. Preliminary estimates of the export of omega-3 highly unsaturated fatty acids (EPA+ DHA) from aquatic to terrestrial ecosystems, p. 179-210. In M. T. Arts, M. T. Brett, and M. Kainz [eds.], Lipids in aquatic ecosystems. Springer.

Gladyshev, M. I., A. Y. Kharitonov, O. N. Popova, N. N. Sushchik, O. N. Makhutova, and G. S. Kalachova. 2011a. Quantitative estimation of dragonfly role in transfer of essential polyunsaturated fatty acids from aquatic to terrestrial ecosystems. Dokl. Biochem. Biophys. 438: $141-143$.

Gladyshev, M. I., V. P. Semenchenko, O. P. Dubovskaya, and others. 2011b. Effect of temperature on contents of essential highly unsaturated fatty acids in freshwater zooplankton. Limnol. Manag. Inl. Waters 41: 339-347.

Gladyshev, M. I., N. N. Sushchik, O. V Anishchenko, O. N. Makhutova, V. I. Kolmakov, G. S. Kalachova, A. A. Kolmakova, and O. P. Dubovskaya. 2011c. Efficiency of transfer of essential polyunsaturated fatty acids versus organic carbon from producers to consumers in a eutrophic reservoir. Oecologia 165: 521-531.

Gladyshev, M. I., N. N. Sushchik, O. P. Dubovskaya, and others. 2015. Fatty acid composition of Cladocera and Copepoda from lakes of contrasting temperature. Freshw. Biol. 60: 373386.

Gladyshev, M. I., N. N. Sushchik, O. N. Makhutova, and others. 2013. Differences in fatty acid composition of cladocerans and copepods from cold and warm lakes. Dokl. Biochem. Biophys. 451: 183-186. 
Gladyshev, M. I., N. N. Sushchik, S. P. Shulepina, A. V Ageev, O. P. Dubovskaya, A. A. Kolmakova, and G. S. Kalachova. 2016. Secondary production of highly unsaturated fatty acids by zoobenthos across rivers contrasting in temperature. River Res. Appl. 32: 12521263.

Gladyshev, M. I., N. N. Sushchik, Y. A. Yurchenko, O. E. Belevich, and G. S. Kalachova. 2011d. Differences in the fatty acid compositions of blood-sucking mosquito larvae and imagoes and the water-to-land export of essential acids. Dokl. Biochem. Biophys. 441: 385388.

Glatz, J. F. C., and J. J. F. P. Luiken. 2015. Fatty acids in cell signaling: Historical perspective and future outlook. Prostag. Leukotr. Ess. 92: 57-62.

Godwin, C. M., and H. J. Carrick. 2008. Spatio-temporal variation of periphyton biomass and accumulation in a temperate spring-fed stream. Aquat. Ecol. 42: 583-595.

Goedkoop, W., M. Demandt, and G. Ahlgren. 2007. Interactions between food quantity and quality (long-chain polyunsaturated fatty acid concentrations) effects on growth and development of Chironomus riparius. Can. J. Fish. Aquat. Sci. 64: 425-436.

Goedkoop, W., and R. K. Johnson. 1996. Pelagic-benthic coupling: Profundal benthic community response to spring diatom deposition in mesotrophic Lake Erken. Limnol. Oceanogr. 41: 636-647.

Golterman, H. L. 1995. The role of the ironhydroxide-phosphate-sulphide system in the phosphate exchange between sediments and overlying water. Hydrobiologia 297: 43-54.

Goulden, C. E., and A. R. Place. 1990. Fatty acid synthesis and accumulation rates in daphniids. J. Exp. Zool. Part A Ecol. Genet. Physiol. 256: 168-178.

Goulden, C. E., and A. R. Place. 1993. Lipid accumulation and allocation in daphniid cladocera. Bull. Mar. Sci. 53: 106-114.

Graham, M. D., and R. D. Vinebrooke. 2009. Extreme weather events alter planktonic communities in boreal lakes. Limnol. Oceanogr. 54: 2481-2492.

Greig, H. S., P. Kratina, P. L. Thompson, W. J. Palen, J. S. Richardson, and J. B. Shurin. 2012. Warming, eutrophication, and predator loss amplify subsidies between aquatic and terrestrial ecosystems. Glob. Chang. Biol. 18: 504-514.

Griffith, M. B., B. H. Hill, A. T. Herlihy, and P. R. Kaufmann. 2002. Multivariate analysis of periphyton assemblages in relation to environmental gradients in Colorado Rocky Mountain streams. J. Phycol. 38: 83-95.

Grimaud, G. M., F. Mairet, A. Sciandra, and O. Bernard. 2017. Modeling the temperature effect on the specific growth rate of phytoplankton: a review. Rev. Environ. Sci. 16: 625-645.

Grimm, N. B., and S. G. Fisher. 1986. Nitrogen limitation in a Sonoran Desert stream. J. North Am. Benthol. Soc. 5: 2-15.

van der Grinten, E., A. P. H. M. Janssen, K. de Mutsert, C. Barranguet, and W. Admiraal. 2005. Temperature-and light-dependent performance of the cyanobacterium Leptolyngbya foveolarum and the diatom Nitzschia perminuta in mixed biofilms. Hydrobiologia 548: 267-278.

Gruendling, G. K. 1971. Ecology of the epipelic algal communities in Marion Lake, British Columbia. J. Phycol. 7: 239-249.

Guariento, R. D., L. S. Carneiro, A. Caliman, R. L. Bozelli, and F. A. Esteves. 2011. How light and nutrients affect the relationship between autotrophic and heterotrophic biomass in a tropical black water periphyton community. Aquat. Ecol. 45: 561-569. 
Guedes, A. C., H. M. Amaro, C. R. Barbosa, R. D. Pereira, and F. X. Malcata. 2011. Fatty acid composition of several wild microalgae and cyanobacteria, with a focus on eicosapentaenoic, docosahexaenoic and $\alpha$-linolenic acids for eventual dietary uses. Food Res. Int. 44: 2721-2729.

Gugger, M., C. Lyra, I. Suominen, I. Tsitko, J. F. Humbert, M. S. Salkinoja-Salonen, and K. Sivonen. 2002. Cellular fatty acids as chemotaxonomic markers of the genera Anabaena, Aphanizomenon, Microcystis, Nostoc and Planktothrix (cyanobacteria). Int. J. Syst. Evol. Microbiol. 52: 1007-1015.

Gulati, R., and W. Demott. 1997. The role of food quality for zooplankton: remarks on the stateof-the-art, perspectives and priorities. Freshw. Biol. 38: 753-768.

Guo, F., M. J. Kainz, F. Sheldon, and S. E. Bunn. 2015. Spatial variation in periphyton fatty acid composition in subtropical streams. Freshw. Biol. 60: 1411-1422.

Guo, F., M. J. Kainz, F. Sheldon, and S. E. Bunn. 2016a. Effects of light and nutrients on periphyton and the fatty acid composition and somatic growth of invertebrate grazers in subtropical streams. Oecologia 181: 449-462.

Guo, F., M. J. Kainz, F. Sheldon, and S. E. Bunn. 2016b. The importance of high-quality algal food sources in stream food webs - current status and future perspectives. Freshw. Biol. 61: 815-831.

Guo, F., M. J. Kainz, D. Valdez, F. Sheldon, and S. E. Bunn. 2016c. High-quality algae attached to leaf litter boost invertebrate shredder growth. Freshw. Sci. 35: 1213-1221.

Guschina, I. A., and J. L. Harwood. 2006. Lipids and lipid metabolism in eukaryotic algae. Prog. Lipid Res. 45: 160-186.

Haack, T. K., and G. A. McFeters. 1982a. Nutritional relationships among microorganisms in an epilithic biofilm community. Microb. Ecol. 8: 115-126.

Haack, T. K., and G. A. McFeters. 1982b. Microbial dynamics of an epilithic mat community in a high alpine stream. Appl. Environ. Microbiol. 43: 702-707.

Hagerthey, S. E., and W. C. Kerfoot. 1998. Groundwater flow influences the biomass and nutrient ratios of epibenthic algae in a north temperate seepage lake. Limnol. Oceanogr. 43: 1227-1242.

Hampton, S. E., E. V. A. Izmest, R. Lyubov, M. V Moore, S. L. Katz, B. Dennis, and E. A. Silow. 2008. Sixty years of environmental change in the world's largest freshwater lakeLake Baikal, Siberia. Glob. Chang. Biol. 14: 1947-1958.

Hanson, B. J., K. W. Cummins, A. S. Cargill, and R. R. Lowry. 1983. Dietary effects on lipid and fatty acid composition of Clistoronia magnifica (Trichoptera: Limnephilidae). Freshw. Invertebr. Biol. 2: 2-15.

Hanson, B. J., K. W. Cummins, A. S. Cargill, and R. R. Lowry. 1985. Lipid content, fatty acid composition, and the effect of diet on fats of aquatic insects. Comp. Biochem. Physiol. Part B Comp. Biochem. 80: 257-276.

Hansson, L. 1988. Effects of competitive interactions on the biomass development of planktonic and periphytic algae in lakes. Limnol. Oceanogr. 33: 121-128.

Hansson, L. 1989. Influence of a periphytic biolayer on phosphorus exchange between substrate and water. Arch. fuer Hydrobiol. 115: 21-26.

Hansson, L. 1990. Quantifying the impact of periphytic algae on nutrient availability for phytoplankton. Freshw. Biol. 24: 265-273.

Hansson, L. 1992. Factors regulating periphytic algal biomass. Limnol. Oceanogr. 37: 322-328. 
Happey-Wood, C. M. 1988. Vertical-migration patterns of flagellates in a community of freshwater benthic algae. Hydrobiologia 161: 99-123.

Harrington, G. W., D. H. Beach, J. E. Dunham, and G. G. Holz. 1970. The polyunsaturated fatty acids of marine dinoflagellates. J. Eukaryot. Microbiol. 17: 213-219.

Hartigan, J. A., and P. M. Hartigan. 1985. The dip test of unimodality. Ann. Stat. 13: 70-84.

Hartmann, D. L., A. M. G. K. Tank, M. Rusticucci, and others. 2013. Observations: atmosphere and surface. In T. F. Stocker, D. Qin, G. K. Plattner, M. Tignor, S. K. Allen, J. Boschung, A. Nauels, Y. Xia, V. Bex and P. M. Midgley [eds.], Climate change 2013: The physical science basis. Contribution of working group I to the fifth assessment report of the Intergovernmental Panel on Climate Change. Cambridge University Press.

Havens, K. E., L. A. Bull, G. L. Warren, T. L. Crisman, E. J. Phlips, and J. P. Smith. 1996. Food web structure in a subtropical lake ecosystem. Oikos 75: 20-32.

Havens, K. E., J. Hauxwell, A. C. Tyler, and others. 2001a. Complex interactions between autotrophs in shallow marine and freshwater ecosystems: implications for community responses to nutrient stress. Environ. Pollut. 113: 95-107.

Havens, K. E., A. D. Steinman, and S.J. Hwang. 2001b. Phosphorus uptake by plankton and periphyton in relation to irradiance and phosphate availability in a subtropical lake (Lake Okeechobee, Florida, USA). Arch. für Hydrobiol. 177-201.

Hazel, J. R. 1995. Thermal adaptation in biological membranes: is homeoviscous adaptation the explanation? Annu. Rev. Physiol. 57: 19-42.

Hazel, J. R., and E. E. Williams. 1990. The role of alterations in membrane lipid composition in enabling physiological adaptation of organisms to their physical environment. Prog. Lipid Res. 29: 167-227.

Hecky, R. E., and R. H. Hesslein. 1995. Contributions of benthic algae to lake food webs as revealed by stable isotope analysis. J. North Am. Benthol. Soc. 14: 631-653.

Hecky, R. E., and P. Kilham. 1988. Nutrient limitation of phytoplankton in freshwater and marine environments: a review of recent evidence on the effects of enrichment. Limnol. Oceanogr. 33: 796-822.

Hecky, R. E., H. J. Kling, and G. J. Brunskill. 1986. Seasonality of phytoplankton in relation to silicon cycling and interstitial water circulation in large, shallow lakes of central Canada. Hydrobiologia 138: 117-126.

Hentschel, E. 1916. Biologische Untersuchungen über den tierischen und pflanzlichen Bewuchs im Hamburger Hafen, Meissner in Komm.

Heydorn, A., A. T. Nielsen, M. Hentzer, C. Sternberg, M. Givskov, B. K. Ersbøll, and S. Molin. 2000. Quantification of biofilm structures by the novel computer program COMSTAT. Microbiology 146: 2395-2407.

Hickling, R., D. B. Roy, J. K. Hill, R. Fox, and C. D. Thomas. 2006. The distributions of a wide range of taxonomic groups are expanding polewards. Glob. Chang. Biol. 12: 450-455.

Hickman, M. 1974. Effects of the discharge of thermal effluent from a power station on Lake Wabamun, Alberta, Canada - The epipelic and epipsamic algal communities. Hydrobiologia 45: 199-215.

Hickman, M. 1978a. Ecological studies on the epipelic algal community in five prairie-parkland lakes in central Alberta. Can. J. Bot. 56: 991-1009.

Hickman, M. 1978b. Studies on the epipelic algal community-seasonal changes and standing crops at shallow littoral stations in four lakes. Arch. für Protistenkd. 120: 1-15. 
Hill, W. R. 1996. Effects of Light, p. 121-148. In R. J. Stevenson, M. L. Bothwell, and R. L. Lowe [eds.], Aquatic ecology. Academic Press.

Hill, W. R., and H. L. Boston. 1991. Community development alters photosynthesis-irradiance relations in stream periphyton. Limnol. Oceanogr. 36: 1375-1389.

Hill, W. R., and S. M. Dimick. 2002. Effects of riparian leaf dynamics on periphyton photosynthesis and light utilisation efficiency. Freshw. Biol. 47: 1245-1256.

Hill, W. R., and S. E. Fanta. 2008. Phosphorus and light colimit periphyton growth at subsaturating irradiances. Freshw. Biol. 53: 215-225.

Hill, W. R., S. E. Fanta, and B. J. Roberts. 2009. Quantifying phosphorus and light effects in stream algae. Limnol. Oceanogr. 54: 368-380.

Hill, W. R., and B. C. Harvey. 1990. Periphyton responses to higher trophic levels and light in a shaded stream. Can. J. Fish. Aquat. Sci. 47: 2307-2314.

Hill, W. R., and A. W. Knight. 1987. Experimental analysis of the grazing interaction between a mayfly and stream algae. Ecology 68: 1955-1965.

Hill, W. R., and A. W. Knight. 1988. Nutrient and Light Limitation of Algae in Two Northern California Streams. J. Phycol. 24: 125-132.

Hill, W. R., J. Rinchard, and S. Czesny. 2011. Light, nutrients and the fatty acid composition of stream periphyton. Freshw. Biol. 56: 1825-1836.

Hill, W. R., M. G. Ryon, and E. M. Schilling. 1995. Light limitation in a stream ecosystem: responses by primary producers and consumers. Ecology 76: 1297-1309.

Hillebrand, H. 2009. Meta-Analysis of Grazer Control of Periphyton Biomass Across Aquatic Ecosystems. J. Phycol. 45: 798-806.

Hillebrand, H., G. de Montpellier, and A. Liess. 2004. Effects of macrograzers and light on periphyton stoichiometry. Oikos 106: 93-104.

Hiltunen, M., U. Strandberg, S. J. Taipale, and P. Kankaala. 2015. Taxonomic identity and phytoplankton diet affect fatty acid composition of zooplankton in large lakes with differing dissolved organic carbon concentration. Limnol. Oceanogr. 60: 303-317.

Hines, J., A. Hammrich, D. Steiner, and M. O. Gessner. 2013. A field facility to simulate climate warming and increased nutrient supply in shallow aquatic ecosystems. Oecologia 173: 1169-1178.

Hixson, S. M., and M. T. Arts. 2016. Climate warming is predicted to reduce omega-3, longchain, polyunsaturated fatty acid production in phytoplankton. Glob. Chang. Biol. 22: 2744-2755.

Hixson, S. M., B. Sharma, M. J. Kainz, A. Wacker, and M. T. Arts. 2015. Production, distribution, and abundance of long-chain omega-3 polyunsaturated fatty acids: a fundamental dichotomy between freshwater and terrestrial ecosystems. Environ. Rev. 23: 414-424.

Hobson, K. A., and H. E. Welch. 1995. Cannibalism and trophic structure in a high Arctic lake: insights from stable-isotope analysis. Can. J. Fish. Aquat. Sci. 52: 1195-1201.

Hodoki, Y. 2005. Bacteria biofilm encourages algal immigration onto substrata in lotic systems. Hydrobiologia 539: 27-34.

Hoflmann, J. P. 1990. Dependence of photosynthesis and vitamin B12 uptake on cellular vitamin B12 concentration in the multicellular alga Cladophora glomerata (Chlorophyta). Limnol. Oceanogr. 35: 100-108.

Holton, R. W., H. H. Blecker, and T. S. Stevens. 1968. Fatty acids in blue-green algae: possible relation to phylogenetic position. Science 160: 545-547. 
Honeyfield, D. C., and K. O. Maloney. 2015. Seasonal patterns in stream periphyton fatty acids and community benthic algal composition in six high-quality headwater streams. Hydrobiologia 744: 35-47.

Hopkins, K., B. Moss, and A. B. Gill. 2011. Increased ambient temperature alters the parental care behaviour and reproductive success of the three-spined stickleback (Gasterosteus aculeatus). Environ. Biol. Fish. 90: 121-129.

Horner, R. R., and E. B. Welch. 1981. Stream periphyton development in relation to current velocity and nutrients. Can. J. Fish. Aquat. Sci. 38: 449-457.

Horner, R. R., E. B. Welch, M. R. Seeley, and J. M. Jacoby. 1990. Responses of periphyton to changes in current velocity, suspended sediment and phosphorus concentration. Freshw. Biol. 24: 215-232.

Horner, R. R., E. B. Welch, and R. B. Veenstra. 1983. Development of nuisance periphytic algae in laboratory streams in relation to enrichment and velocity, p. 121-134. In R. G. Wetzel [ed.], Periphyton of freshwater ecosystems. Springer.

Huggins, K., J. Frenette, and M. T. Arts. 2004. Nutritional quality of biofilms with respect to light regime in Lake Saint-Pierre (Québec, Canada). Freshw. Biol. 49: 945-959.

Hunter, R. D. 1980. Effects of grazing on the quantity and quality of freshwater aufwuchs. Hydrobiologia 69: 251-259.

Huntsman, A. G. 1948. Fertility and fertilization of streams. J. Fish. Board Canada 7: 248-253.

Hustedt, F. 1956. Kieselalgen (Diatomeen), Kosmos, Gesellschaft der Naturfreunde Franckh'sche Verlagshandlung.

Hwang, S. J., K. E. Havens, and A. D. Steinman. 1998. Phosphorus kinetics of planktonic and benthic assemblages in a shallow subtropical lake. Freshw. Biol. 40: 729-745.

Ibarguren, M., D. J. López, and P. V Escribá. 2014. The effect of natural and synthetic fatty acids on membrane structure, microdomain organization, cellular functions and human health. Biochim. Biophys. Acta. 1838: 1518-1528.

Iliev, I., G. Petkov, J. Lukavsky, S. Furnadzhieva, and R. Andreeva. 2011. Do cyanobacterial lipids contain fatty acids longer than 18 carbon atoms? Z. Naturforsch. C. 66: 267-276.

Indarti, E., M. I. A. Majid, R. Hashim, and A. Chong. 2005. Direct FAME synthesis for rapid total lipid analysis from fish oil and cod liver oil. J. Food Compos. Anal. 18: 161-170.

Jacoby, J. M. 1985. Grazing effects on periphyton by Theodoxus fluviatilis (Gastropoda) in a lowland stream. J. Freshw. Ecol. 3: 265-274.

Jäger, C. G., and D. Borchardt. 2018. Longitudinal patterns and response lengths of algae in riverine ecosystems: a model analysis emphasising benthic-pelagic interactions. J. Theor. Biol. 442: 66-78.

Jain, A., and N. B. Bhosle. 2009. Biochemical composition of the marine conditioning film: implications for bacterial adhesion. Biofouling 25: 13-19.

James, M. R., I. Hawes, M. Weatherhead, C. Stanger, and M. Gibbs. 2000. Carbon flow in the littoral food web of an oligotrophic lake. Hydrobiologia 441: 93-106.

James, M. R., M. Weatherhead, C. Stanger, and E. Graynoth. 1998. Macroinvertebrate distribution in the littoral zone of Lake Coleridge, South Island, New Zealand - effects of habitat stability, wind exposure, and macrophytes. New Zeal. J. Mar. Freshw. Res. 32: 287305.

Jasser, I., and I. Kostrzewska-Szlakowska. 2012. Fading out of the trophic cascade at the base of the microbial food web caused by changes in the grazing community in mesocosm experiments. Oceanol. Hydrobiol. Stud. 41: 1-11. 
Jiang, X., X. Jin, Y. Yao, L. Li, and F. Wu. 2008. Effects of biological activity, light, temperature and oxygen on phosphorus release processes at the sediment and water interface of Taihu Lake, China. Water Res. 42: 2251-2259.

Joehnk, K. D., J. Huisman, J. Sharples, B. E. N. Sommeijer, P. M. Visser, and J. M. Stroom. 2008. Summer heatwaves promote blooms of harmful cyanobacteria. Glob. Chang. Biol. 14: 495-512.

Jones, J. I., J. W. Eaton, and K. Hardwick. 2000. The influence of periphyton on boundary layer conditions: a pH microelectrode investigation. Aquat. Bot. 67: 191-206.

Jones, J. I., J. O. Young, J. W. Eaton, and B. Moss. 2002. The influence of nutrient loading, dissolved inorganic carbon and higher trophic levels on the interaction between submerged plants and periphyton. J. Ecol. 90: 12-24.

Jones, S. E., and M. A. Lock. 1993. Seasonal determinations of extracellular hydrolytic activities in heterotrophic and mixed heterotrophic/autotrophic biofilms from two contrasting rivers. Hydrobiologia 257: 1-16.

Juttner, F., and J. T. Wu. 2000. Evidence of allelochemical activity in subtropical cyanobacterial biofilms of Taiwan. Arch. für Hydrobiol. 505-517.

Kahlert, M., A. T. Hasselrot, H. Hillebrand, and K. Pettersson. 2002. Spatial and temporal variation in the biomass and nutrient status of epilithic algae in Lake Erken, Sweden. Freshw. Biol. 47: 1191-1215.

Kainz, M. J., M. T. Arts, and A. Mazumder. 2004. Essential fatty acids in the planktonic food web and their ecological role for higher trophic levels. Limnol. Oceanogr. 49: 1784-1793.

Kalachova, G. S., M. I. Gladyshev, N. N. Sushchik, O. P. Dubovskaya, S. P. Shulepina, and A. V Ageev. 2013. Production of polyunsaturated fatty acids by zoobenthos in rivers with contrasting temperatures. Dokl. Biochem. Biophys. 453: 308-311.

Kankaala, P., A. Ojala, T. Tulonen, J. Haapamäki, and L. Arvola. 2000. Response of littoral vegetation on climate warming in the boreal zone; an experimental simulation. Aquat. Ecol. 34: 433-444.

Karentz, D., and L. H. Lutze. 1990. Evaluation ofbiologically harmful ultraviolet radiation in Antarctica with a biological dosimeter designed for aquatic environments. Limnol. Oceanogr. 35: 549-561.

Kates, M., and B. E. Volcani. 1966. Lipid components of diatoms. Biochim. Biophys. Acta. 116: 264-278.

Kenyon, C. N., R. Rippka, and R. Y. Stanier. 1972. Fatty acid composition and physiological properties of some filamentous blue-green algae. Arch. Mikrobiol. 83: 216-236.

Kevern, N. R., and R. C. Ball. 1965. Primary productivity and energy relationships in artificial streams. Limnol. Oceanogr. 10: 74-87.

Khondker, M., and M. Dokulil. 1988. Seasonality, biomass and primary productivity of epipelic algae in a shallow lake (Neusiedlersee, Austria). Acta. Hydroch. Hydrob. 16: 499-515.

Kiffney, P. M., and W. H. Clements. 1993. Bioaccumulation of heavy metals by benthic invertebrates at the Arkansas River, Colorado. Environ. Toxicol. Chem. 12: 1507-1517.

Kiffney, P. M., J. S. Richardson, and J. P. Bull. 2003. Responses of periphyton and insects to experimental manipulation of riparian buffer width along forest streams. J. Appl. Ecol. 40: 1060-1076.

Kiffney, P. M., J. S. Richardson, and J. P. Bull. 2004. Establishing light as a causal mechanism structuring stream communities in response to experimental manipulation of riparian buffer width. J. North Am. Benthol. Soc. 23: 542-555. 
Kilham, S. S., D. A. Kreeger, S. G. Lynn, C. E. Goulden, and L. Herrera. 1998. COMBO: a defined freshwater culture medium for algae and zooplankton. Hydrobiologia 377: 147159.

Kim, Y., and S. H. Kim. 2009. Released exopolysaccharide (r-EPS) produced from probiotic bacteria reduce biofilm formation of enterohemorrhagic Escherichia coli O157: H7. Biochem. Biophys. Res. Commun. 379: 324-329.

Kirk, J. T. O. 1994. Light and photosynthesis in aquatic ecosystems, $2^{\text {nd }}$ ed. Cambridge University Press.

Klarer, D. M., and M. Hickman. 1975. The effect of thermal effluent upon the standing crop of an epiphytic algal community. Int. Rev. der gesamten Hydrobiol. und Hydrogr. 60: 17-62.

Kline Jr, T. C., J. J. Goering, O. A. Mathisen, P. H. Poe, P. L. Parker, and R. S. Scalan. 1993. Recycling of elements transported upstream by runs of Pacific salmon: II. $\delta^{15} \mathrm{~N}$ and $\delta^{13} \mathrm{C}$ evidence in the Kvichak River watershed, Bristol Bay, southwestern Alaska. Can. J. Fish. Aquat. Sci. 50: 2350-2365.

Kohler, T. J., T. N. Heatherly, R. W. El-Sabaawi, and others. 2012. Flow, nutrients, and light availability influence Neotropical epilithon biomass and stoichiometry. Freshw. Sci. 31: 1019-1034.

Koussoroplis, A. M., J. Nussbaumer, M. T. Arts, I. A. Guschina, and M. J. Kainz. 2014. Famine and feast in a common freshwater calanoid: Effects of diet and temperature on fatty acid dynamics of Eudiaptomus gracilis. Limnol. Oceanogr. 59: 947-958.

Kovacevic, V., A. J. Simpson, and M. J. Simpson. 2018. Investigation of Daphnia magna sublethal exposure to organophosphate esters in the presence of dissolved organic matter using ${ }^{1} \mathrm{H}$ NMR-based metabolomics. Metabolites 8: 34 .

Kreuzinger-Janik, B., F. Schroeder, N. Majdi, and W. Traunspurger. 2015. Depth-related effects on a meiofaunal community dwelling in the periphyton of a mesotrophic lake. PLoS One 10: e0137793.

Kühl, M., and B. B. Jorgensen. 1994. The light field of microbenthic communities: radiance distribution and microscale optics of sandy coastal sediments. Limnol. Oceanogr. 39: 13681398.

Kühl, M., and L. Polerecky. 2008. Functional and structural imaging of phototrophic microbial communities and symbioses. Aquat. Microb. Ecol. 53: 99-118.

Kundzewicz, Z. W., L. J. Mata, N. W. Arnell, and others. 2008. The implications of projected climate change for freshwater resources and their management. Hydrol. Sci. J. 53: 3-10.

Lall, S. P., and L. M. Lewis-McCrea. 2007. Role of nutrients in skeletal metabolism and pathology in fish - an overview. Aquaculture 267: 3-19.

Lalonde, S., and J. A. Downing. 1991. Epiphyton biomass is related to lake trophic status, depth, and macrophyte architecture. Can. J. Fish. Aquat. Sci. 48: 2285-2291.

Lamberti, G. A., J. W. Feminella, and V. H. Resh. 1987. Herbivory and intraspecific competition in a stream caddisfly population. Oecologia 73 : $75-81$.

Lamberti, G. A., and V. H. Resh. 1983. Stream periphyton and insect herbivores: an experimental study of grazing by a caddisfly population. Ecology 64: 1124-1135.

Lamberti, G. A., and V. H. Resh. 1985. Distribution of benthic algae and macroinvertebrates along a thermal stream gradient. Hydrobiologia 128: 13-21.

Lang, I., L. Hodac, T. Friedl, and I. Feussner. 2011. Fatty acid profiles and their distribution patterns in microalgae: a comprehensive analysis of more than 2000 strains from the SAG culture collection. BMC Plant Biol. 11: 124. 
Langdon, C. 1988. On the causes of interspecific differences in the growth-irradiance relationship for phytoplankton. II. A general review. J. Plankton Res. 10: 1291-1312.

Larned, S. T., and S. R. Santos. 2000. Light-and nutrient-limited periphyton in low order streams of Oahu, Hawaii. Hydrobiologia 432: 101-111.

Larras, F., A. S. Lambert, S. Pesce, F. Rimet, A. Bouchez, and B. Montuelle. 2013. The effect of temperature and a herbicide mixture on freshwater periphytic algae. Ecotoxicol. Environ. Saf. 98: 162-170.

Lau, D. C. P., K. M. Y. Leung, and D. Dudgeon. 2009. Are autochthonous foods more important than allochthonous resources to benthic consumers in tropical headwater streams? J. North Am. Benthol. Soc. 28: 426-439.

Laviale, M., J. Prygiel, Y. Lemoine, A. Courseaux, and A. Creach. 2009. Stream Periphyton Photoacclimation Response in Field Conditions: Effect of Community Development and Seasonal Changes. J. Phycol. 45: 1072-1082.

Lawrence, J. R., M. R. Chenier, R. Roy, D. Beaumier, N. Fortin, G. D. W. Swerhone, T. R. Neu, and C. W. Greer. 2004. Microscale and molecular assessment of impacts of nickel, nutrients, and oxygen level on structure and function of river biofilm communities. Appl. Environ. Microbiol. 70: 4326-4339.

Lawrence, J. R., D. R. Korber, and T. R. Neu. 2007. Analytical imaging and microscopy techniques, p. 40-68. In C. Hurst, R. Crawford, J. Garland, D. Lipson, A. Mills, and L. Stetzenbach [eds.], Manual of environmental microbiology, third edition. ASM Press.

Lawrence, J. R., B. Scharf, G. Packroff, and T. R. Neu. 2002. Microscale evaluation of the effects of grazing by invertebrates with contrasting feeding modes on river biofilm architecture and composition. Microb. Ecol. 44: 199-207.

Lee, R. F., J. C. Nevenzel, and G. A. Paffenhöfer. 1971. Importance of wax esters and other lipids in the marine food chain: phytoplankton and copepods. Mar. Biol. 9: 99-108.

Leflaive, J., S. Boulêtreau, E. Buffan-Dubau, and L. Ten-Hage. 2008. Temporal patterns in epilithic biofilm-relation with a putative allelopathic activity. Fundam. Appl. Limnol. für Hydrobiol. 173: 121-134.

Leflaive, J., and L. Ten-Hage. 2009. Allelopathic interactions in benthic biofilms: effects of abiotic conditions on production of and sensitivity to allelochemicals. J. North Am. Benthol. Soc. 28: 273-282.

Leguay, S., I. Lavoie, J. L. Levy, and C. Fortin. 2016. Using biofilms for monitoring metal contamination in lotic ecosystems: The protective effects of hardness and $\mathrm{pH}$ on metal bioaccumulation. Environ. Toxicol. Chem. 35: 1489-1501.

Lem, N. W., and P. K. Stumpf. 1984. In vitro fatty acid synthesis and complex lipid metabolism in the cyanobacterium Anabaena variabilis: I. Some characteristics of fatty acid synthesis. Plant Physiol. 74: 134-138.

Lennon, J. T., V. H. Smith, and A. R. Dzialowski. 2003. Invasibility of plankton food webs along a trophic state gradient. Oikos 103: 191-203.

Lenth, R. V. 2016. Least-squares means: the R package lsmeans. J. Stat. Softw. 69: 1-33.

Leu, E., J. Wiktor, J. E. Søreide, J. Berge, and S. Falk-Petersen. 2010. Increased irradiance reduces food quality of sea ice algae. Mar. Ecol. Prog. Ser. 411: 49-60.

Li-Beisson, Y., F. Beisson, and W. Riekhof. 2015. Metabolism of acyl-lipids in Chlamydomonas reinhardtii. Plant J. 82: 504-522. 
Li, W. K. W. 1980. Temperature adaptation in phytoplankton: cellular and photosynthetic characteristics, p. 259-279. In P. G. Falkowski [ed.], Primary productivity in the sea. Springer.

Libkind, D., M. T. Arts, and M. Van Broock. 2008. Fatty acid composition of cold-adapted carotenogenic basidiomycetous yeasts. Rev Argent Microbiol 40: 193-197.

Liboriussen, L., and E. Jeppesen. 2006. Structure, biomass, production and depth distribution of periphyton on artificial substratum in shallow lakes with contrasting nutrient concentrations. Freshw. Biol. 51: 95-109.

Liboriussen, L., and E. Jeppesen. 2009. Periphyton biomass, potential production and respiration in a shallow lake during winter and spring. Hydrobiologia 632: 201-210.

Liboriussen, L., F. Landkildehus, M. Meerhoff, and others. 2005. Global warming: Design of a flow-through shallow lake mesocosm climate experiment. Limnol. Oceanogr. Methods 3: $1-9$.

Liess, A., K. Lange, F. Schulz, J. J. Piggott, C. D. Matthaei, and C. R. Townsend. 2009. Light, nutrients and grazing interact to determine diatom species richness via changes to productivity, nutrient state and grazer activity. J. Ecol. 97: 326-336.

Lin, S., R. W. Litaker, and W. G. Sunda. 2016. Phosphorus physiological ecology and molecular mechanisms in marine phytoplankton. J. Phycol. 52: 10-36.

Liu, J., R. Martinez-Corral, A. Prindle, D. L. Dong-yeon, J. Larkin, M. Gabalda-Sagarra, J. Garcia-Ojalvo, and G. M. Süel. 2017. Coupling between distant biofilms and emergence of nutrient time-sharing. Science 356: 638-642.

Liu, J., A. Prindle, J. Humphries, and others. 2015. Metabolic co-dependence gives rise to collective oscillations within biofilms. Nature 523: 550.

Lohman, K., J. R. Jones, and C. Baysinger-Daniel. 1991. Experimental evidence for nitrogen limitation in a northern Ozark stream. J. North Am. Benthol. Soc. 10: 14-23.

Los, D. A., and K. S. Mironov. 2015. Modes of fatty acid desaturation in cyanobacteria: an update. Life 5: 554-567.

Los, D. A., and N. Murata. 2004. Membrane fluidity and its roles in the perception of environmental signals. Biochim. Biophys. Acta 1666: 142-157.

Losee, R. F., and R. G. Wetzel. 1983. Selective light attenuation by the periphyton complex, p. 89-96. In R. G. Wetzel [ed.], Periphyton of freshwater ecosystems. Springer.

Lowe, R. L., S. W. Golladay, and J. R. Webster. 1986. Periphyton response to nutrient manipulation in streams draining clearcut and forested watersheds. J. North Am. Benthol. Soc. 5: 221-229.

Lürling, M., F. Eshetu, E. J. Faassen, S. Kosten, and V. L. M. Huszar. 2013. Comparison of cyanobacterial and green algal growth rates at different temperatures. Freshw. Biol. 58: $552-559$.

Lyon, D. R., and S. E. Ziegler. 2009. Carbon cycling within epilithic biofilm communities across a nutrient gradient of headwater streams. Limnol. Oceanogr. 54: 439-449.

Maazouzi, C., G. Masson, M. S. Izquierdo, and J. C. Pihan. 2008. Midsummer heat wave effects on lacustrine plankton: variation of assemblage structure and fatty acid composition. J. Therm. Biol. 33: 287-296.

Mack, W. N., J. P. Mack, and A. O. Ackerson. 1975. Microbial film development in a trickling filter. Microb. Ecol. 2: 215-226.

MacLeod, J. C., and E. Pessah. 1973. Temperature effects on mercury accumulation, toxicity, and metabolic rate in rainbow trout (Salmo gairdneri). J. Fish. Board Canada 30: 485-492. 
Madsen, J. S., M. Burmølle, L. H. Hansen, and S. J. Sørensen. 2012. The interconnection between biofilm formation and horizontal gene transfer. FEMS Immunol. Med. Microbiol. 65: $183-195$.

Maechler, M. 2015. Diptest: Hartigan's dip test statistic for unimodality—corrected. R package version 0.75-7. https://CRAN.R-project.org/package=diptest

Mah, T. F., B. Pitts, B. Pellock, G. C. Walker, P. S. Stewart, and G. A. O’Toole. 2003. A genetic basis for Pseudomonas aeruginosa biofilm antibiotic resistance. Nature 426: 306-311.

Mahdy, A., S. Hilt, N. Filiz, and others. 2015. Effects of water temperature on summer periphyton biomass in shallow lakes: a pan-European mesocosm experiment. Aquat. Sci. 77: 499-510.

Majdi, N., W. Traunspurger, S. Boyer, and others. 2011. Response of biofilm-dwelling nematodes to habitat changes in the Garonne River, France: influence of hydrodynamics and microalgal availability. Hydrobiologia 673: 229-244.

Makhutova, O. N., E. V Borisova, S. P. Shulepina, A. A. Kolmakova, and N. N. Sushchik. 2017. Fatty acid composition and content in chironomid species at various life stages dominating in a saline Siberian lake. Contemp. Probl. Ecol. 10: 230-239.

Makhutova, O. N., N. N. Sushchik, M. I. Gladyshev, A. V Ageev, E. G. Pryanichnikova, and G. S. Kalachova. 2011. Is the fatty acid composition of freshwater zoobenthic invertebrates controlled by phylogenetic or trophic factors? Lipids 46: 709-721.

Marker, A. F. H. 1976. The benthic algae of some streams in Southern England: I. Biomass of the epilithon in some small streams. J. Ecol. 64: 343-358.

Marks, J. C., and R. L. Lowe. 1989. The independent and interactive effects of snail grazing and nutrient enrichment on structuring periphyton communities. Hydrobiologia 185: 9-17.

Marks, J. C., and R. L. Lowe. 1993. Interactive effects of nutrient availability and light levels on the periphyton composition of a large oligotrophic lake. Can. J. Fish. Aquat. Sci. 50: 12701278.

Martin-Creuzburg, D., E. von Elert, and K. H. Hoffmann. 2008. Nutritional constraints at the cyanobacteria-Daphnia magna interface: the role of sterols. Limnol. Oceanogr. 53: 456468.

Martin-Creuzburg, D., C. Kowarik, and D. Straile. 2017. Cross-ecosystem fluxes: Export of polyunsaturated fatty acids from aquatic to terrestrial ecosystems via emerging insects. Sci. Total Environ. 577: 174-182.

Martínez, A., A. Larrañaga, J. Pérez, E. Descals, and J. Pozo. 2014. Temperature affects leaf litter decomposition in low-order forest streams: field and microcosm approaches. FEMS Microbiol. Ecol. 87: 257-267.

Masclaux, H., A. Bec, M. J. Kainz, F. Perriere, C. Desvilettes, and G. Bourdier. 2012. Accumulation of polyunsaturated fatty acids by cladocerans: effects of taxonomy, temperature and food. Freshw. Biol. 57: 696-703.

Mason, C. F., and R. J. Bryant. 1975. Periphyton production and grazing by chironomids in Alderfen Broad, Norfolk. Freshw. Biol. 5: 271-277.

Matz, C., D. McDougald, A. M. Moreno, P. Y. Yung, F. H. Yildiz, and S. Kjelleberg. 2005. Biofilm formation and phenotypic variation enhance predation-driven persistence of Vibrio cholerae. Proc. Natl. Acad. Sci. U. S. A. 102: 16819-16824.

Mazumder, A., W. D. Taylor, D. J. McQueen, and D. R. S. Lean. 1989. Effects of nutrients and grazers on periphyton phosphorus in lake enclosures. Freshw. Biol. 22: 405-415. 
McCombie, A. M. 1959. Some relations between air temperatures and the surface water temperatures of lakes. Limnol. Oceanogr. 4: 252-258.

McCormick, P. V, R. B. E. Shuford III, J. G. Backus, and W. C. Kennedy. 1997. Spatial and seasonal patterns of periphyton biomass and productivity in the northern Everglades, Florida, USA. Hydrobiologia 362: 185-210.

McCree, K. J. 1981. Photosynthetically active radiation, p. 41-55. In O. L. Lange, P. S. Nobel, C. B. Osmond and H. Ziegler [eds.], Physiological plant ecology I. Springer.

McIntire, C. D. 1966. Some factors affecting respiration of periphyton communities in lotic environments. Ecology 47: 918-930.

McIntire, C. D., and H. K. Phinney. 1965. Laboratory studies of periphyton production and community metabolism in lotic environments. Ecol. Monogr. 35: 237-258.

McIntire, C. D., I. J. Tinsley, and R. R. Lowry. 1969. Fatty acids in lotic periphyton: another measure of community structure. J. Phycol. 5: 26-32.

McKee, D., D. Atkinson, S. E. Collings, and others. 2000. Heated aquatic microcosms for climate change experiments. Freshwater Forum. 14: 51-58.

McKee, D., D. Atkinson, S. E. Collings, and others. 2002a. Macro-zooplankter responses to simulated climate warming in experimental freshwater microcosms. Freshw. Biol. 47: 1557-1570.

McKee, D., D. Atkinson, S. E. Collings, and others. 2003. Response of freshwater microcosm communities to nutrients, fish, and elevated temperature during winter and summer. Limnol. Oceanogr. 48: 707-722.

McKee, D., K. Hatton, J. W. Eaton, D. Atkinson, A. Atherton, I. Harvey, and B. Moss. 2002 b. Effects of simulated climate warming on macrophytes in freshwater microcosm communities. Aquat. Bot. 74: 71-83.

Melbourne, B. A., and P. J. Daniel. 2003. A low-cost sensor for measuring spatiotemporal variation of light intensity on the streambed. J. North Am. Benthol. Soc. 22: 143-151.

Meulemans, J. T. 1987. A method for measuring selective light attenuation within a periphytic community. Arch. für Hydrobiol. Stuttgart 109: 139-145.

Mialet, B., N. Majdi, M. Tackx, F. Azémar, and E. Buffan-Dubau. 2013. Selective feeding of bdelloid rotifers in river biofilms. PLoS One 8: e75352.

Middelburg, J. J. 2014. Stable isotopes dissect aquatic food webs from the top to the bottom. Biogeosciences 11: 2357-2371.

Mills, K. H., S. M. Chalanchuk, P. J. Blanchfield, C. L. Podemski, and D. J. Allan. 2008. Enhanced growth and condition of lake trout in a small Ontario lake during cage aquaculture of rainbow trout. Can. Tech. Rep. Fish. Aquat. Sci 2778: 200-202.

Mishra, G. 2015. Polyunsaturated fatty acids from algae, p. 467-481. In D. Sahoo and J. Seckbach [eds.], The algae world. Springer.

Mitbavkar, S., and A. C. Anil. 2007. Species interactions within a fouling diatom community: roles of nutrients, initial inoculum and competitive strategies. Biofouling 23: 99-112.

Moccia, R. D., and D. J. Bevan. 2018. AQUASTATS: Ontario Aquacultural Production in 2017. University of Guelph.

Mock, T., and B. M. A. Kroon. 2002. Photosynthetic energy conversion under extreme conditions-II: the significance of lipids under light limited growth in Antarctic sea ice diatoms. Phytochemistry 61: 53-60. 
Molin, S., and T. Tolker-Nielsen. 2003. Gene transfer occurs with enhanced efficiency in biofilms and induces enhanced stabilisation of the biofilm structure. Curr. Opin. Biotechnol. 14: $255-261$.

Montagnes, D. J. S., S. A. Kimmance, and D. Wilson. 2002. Effects of global and local temperature changes on free living, aquatic protists. In C. L. Bolis, R. Keines, M. Elia [eds.], Conference proceedings of the 14th International Conference on Comparative Physiology, climate changes: effects on plants, animals, and humans.

Moody, E. K., A. T. Rugenski, J. L. Sabo, B. L. Turner, and J. J. Elser. 2017. Does the Growth Rate Hypothesis Apply across Temperatures? Variation in the Growth Rate and Body Phosphorus of Neotropical Benthic Grazers. Front. Environ. Sci. 5: 14.

Mooney, B. D., P. D. Nichols, M. F. de Salas, and G. M. Hallegraeff. 2007. Lipid, Fatty Acid, and Sterol Composition of Eight Species of Kareniaceae (Dinophyta): Chemotaxonomy and Putative Lipid Phycotoxins. J. Phycol. 43: 101-111.

Moore, J. W. 1977a. Seasonal succession of algae in a eutrophic stream in southern England. Hydrobiologia 53: 181-192.

Moore, J. W. 1977b. Seasonal succession of algae in rivers. 2. Examples from highland water, a small woodland stream. Arch. für Hydrobiol. 80: 160-171.

Moore, L. F. 1978. Attached algae at thermal generating stations - the effect of temperature on Cladophora. Int. Vereinigung für Theor. und Angew. Limnol. Verhandlungen 20: 17271733.

Mora-Gómez, J., A. Freixa, N. Perujo, and L. Barral-Fraga. 2016. Limits of the biofilm concept and types of aquatic biofilms, p. 3-28. In A. M. Romaní, H. Guasch, and M. D. Balaguer [eds.], Aquatic biofilms: Ecology, water quality and wastewater treatment. Caister Academic Press.

Moran, R., I. Harvey, B. Moss, H. Feuchtmayr, K. Hatton, T. Heyes, and D. Atkinson. 2010. Influence of simulated climate change and eutrophication on three-spined stickleback populations: a large scale mesocosm experiment. Freshw. Biol. 55: 315-325.

Moreno, V. J., J. E. A. Moreno, and R. R. Brenner. 1979. Fatty acid metabolism of the calanoid copepod Paracalanus parvus: 2. Palmitate, stearate, oleate and acetate. Lipids 14: 318-322.

Morin, A., and A. Cattaneo. 1992. Factors affecting sampling variability of freshwater periphyton and the power of periphyton studies. Can. J. Fish. Aquat. Sci. 49: 1695-1703.

Morin, A., W. Lamoureux, and J. Busnarda. 1999. Empirical models predicting primary productivity from chlorophyll a and water temperature for stream periphyton and lake and ocean phytoplankton. J. North Am. Benthol. Soc. 18: 299-307.

Morrill, J. C., R. C. Bales, and M. H. Conklin. 2005. Estimating stream temperature from air temperature: implications for future water quality. J. Environ. Eng. 131: 139-146.

Mortain-Bertrand, A., C. Descolas-Gros, and H. Jupin. 1988. Growth, photosynthesis and carbon metabolism in the temperate marine diatom Skeletonema costatum adapted to low temperature and low photon-flux density. Mar. Biol. 100: 135-141.

Mortensen, S. H., K. Y. Børsheim, J. Rainuzzo, and G. Knutsen. 1988. Fatty acid and elemental composition of the marine diatom Chaetoceros gracilis Schütt. Effects of silicate deprivation, temperature and light intensity. J. Exp. Mar. Bio. Ecol. 122: 173-185.

Mosisch, T. D., S. E. Bunn, and P. M. Davies. 2001. The relative importance of shading and nutrients on algal production in subtropical streams. Freshw. Biol. 46: 1269-1278. 
Moss, B., D. McKee, D. Atkinson, and others. 2003. How important is climate? Effects of warming, nutrient addition and fish on phytoplankton in shallow lake microcosms. J. Appl. Ecol. 40: 782-792.

Mõttus, M., M. Sulev, B. Frederic, R. Lopez-Lozano, and A. Reinart. 2011. Photosynthetically Active Radiation: Measurement and Modeling, p. 7902-7932. In C. Richter, D. Lincot, and C. A. Gueymard [eds.], Solar energy. Springer.

Mouget, J. L., A. Dakhama, M. C. Lavoie, and J. de la Noüe. 1995. Algal growth enhancement by bacteria: is consumption of photosynthetic oxygen involved? FEMS Microbiol. Ecol. 18: $35-43$.

Mozaffarian, D. 2009. Fish, mercury, selenium and cardiovascular risk: current evidence and unanswered questions. Int. J. Environ. Res. Public Health 6: 1894-1916.

Mueller-Navarra, D. 1995. Evidence that a highly unsaturated fatty acid limits Daphnia growth in nature. Arch. für Hydrobiol. 132: 297-307.

Mueller, L. N., J. F. C. de Brouwer, J. S. Almeida, L. J. Stal, and J. B. Xavier. 2006. Analysis of a marine phototrophic biofilm by confocal laser scanning microscopy using the new image quantification software PHLIP. BMC Ecol. 6: 1, doi: 10.1186/1472-6785-6-1.

Mulholland, P. J., E. R. Marzolf, S. P. Hendricks, R. V Wilkerson, and A. K. Baybayan. 1995. Longitudinal patterns of nutrient cycling and periphyton characteristics in streams: a test of upstream-downstream linkage. J. North Am. Benthol. Soc. 14: 357-370.

Mulholland, P. J., A. D. Steinman, A. V Palumbo, J. W. Elwood, and D. B. Kirschtel. 1991. Role of nutrient cycling and herbivory in regulating periphyton communities in laboratory streams. Ecology 72: 966-982.

Müller-Navarra, D. C., M. T. Brett, A. M. Liston, and C. R. Goldman. 2000. A highly unsaturated fatty acid predicts carbon transfer between primary producers and consumers. Nature 403: 74.

Munawar, M., and I. F. Munawar. 1986. The seasonality of phytoplankton in the North American Great Lakes, a comparative synthesis, p. 85-115. In M. Munawar and J. F. Talling [eds.], Seasonality of freshwater phytoplankton. Springer.

Murata, N., H. Wada, and Z. Gombos. 1992. Modes of fatty-acid desaturation in cyanobacteria. Plant Cell Physiol. 33: 933-941.

Murga, R., P. S. Stewart, and D. Daly. 1995. Quantitative analysis of biofilm thickness variability. Biotechnol. Bioeng. 45: 503-510.

Murray, R. E., K. E. Cooksey, and J. C. Priscu. 1986. Stimulation of bacterial DNA synthesis by algal exudates in attached algal-bacterial consortia. Appl. Environ. Microbiol. 52: 11771182.

Myklestad, S. M. 1995. Release of extracellular products by phytoplankton with special emphasis on polysaccharides. Sci. Total Environ. 165: 155-164.

Neely, R. K., and R. G. Wetzel. 1995. Simultaneous use of ${ }^{14} \mathrm{C}$ and ${ }^{3} \mathrm{H}$ to determine autotrophic production and bacterial protein production in periphyton. Microb. Ecol. 30: 227-237.

Netten, J. J. C., E. H. van Nes, M. Scheffer, and R. M. M. Roijackers. 2008. Use of open-top chambers to study the effect of climate change in aquatic ecosystems. Limnol. Oceanogr. Methods 6: 223-229.

Neu, T. R., and J. R. Lawrence. 2016. Laser microscopy for the study of biofilms: Issues and options, p. 29-45. In A. M. Romaní, H. Guasch, and M. D. Balaguer [eds.], Aquatic biofilms: Ecology, water quality and wastewater treatment. Caister Academic Press. 
Neu, T. R., G. D. W. Swerhone, U. Böckelmann, and J. R. Lawrence. 2005. Effect of CNP on composition and structure of lotic biofilms as detected with lectin-specific glycoconjugates. Aquat. Microb. Ecol. 38: 283-294.

Neumann, D. 1961. Ernährungsbiologie einer rhipidoglossen Kiemenschnecke. Hydrobiologia 17: $133-151$.

Nicolella, C., M. C. M. van Loosdrecht, and J. J. Heijnen. 2000. Wastewater treatment with particulate biofilm reactors. J. Biotechnol. 80: 1-33.

Nielsen, E. S., and E. G. Jørgensen. 1968. The adaptation of plankton algae. Physiol. Plant. 21: 401-413.

Nishida, I., and N. Murata. 1996. Chilling sensitivity in plants and cyanobacteria: the crucial contribution of membrane lipids. Annu. Rev. Plant Biol. 47: 541-568.

Noffke, N., D. Christian, D. Wacey, and R. M. Hazen. 2013. Microbially induced sedimentary structures recording an ancient ecosystem in the $c a .3 .48$ billion-year-old Dresser Formation, Pilbara, Western Australia. Astrobiology 13: 1103-1124.

Nozaki, K., H. Morino, H. Munehara, V. G. Sideleva, K. Nakai, M. Yamauchi, O. M. Kozhova, and M. Nakanishi. 2002. Composition, biomass, and photosynthetic activity of the benthic algal communities in a littoral zone of lake Baikal in summer. Limnology 3: 175-180.

O'Brien, P. J., and J. D. Wehr. 2009. Periphyton biomass and ecological stoichiometry in streams within an urban to rural land-use gradient, p. 89-105. In R. J. Stevenson and S. Sabater [eds.], Global change and river ecosystems-Implications for structure, function and ecosystem services. Springer.

O’Gorman, E. J., Ó. P. Ólafsson, B. O. L. Demars, and others. 2016. Temperature effects on fish production across a natural thermal gradient. Glob. Chang. Biol. 22: 3206-3220.

O'Gorman, E. J., D. E. Pichler, G. Adams, and others. 2012. Impacts of warming on the structure and functioning of aquatic communities: individual-to ecosystem-level responses. Adv. Ecol. Res. 47: 81-176.

Oehmen, A., P. C. Lemos, G. Carvalho, Z. Yuan, J. Keller, L. L. Blackall, and M. A. M. Reis. 2007. Advances in enhanced biological phosphorus removal: from micro to macro scale. Water Res. 41: 2271-2300.

Oemke, M. P., and T. M. Burton. 1986. Diatom colonization dynamics in a lotic system. Hydrobiologia 139: 153-166.

Oger, P. M., and A. Cario. 2013. Adaptation of the membrane in Archaea. Biophys. Chem. 183: 42-56.

Owen, J. M., J. W. Adron, C. Middleton, and C. B. Cowey. 1975. Elongation and desaturation of dietary fatty acids in turbot Scophthalmus maximus L., and rainbow trout, Salmo gairdnerii rich. Lipids 10: 528-531.

Paerl, H. W., and J. Huisman. 2008. Blooms like it hot. Science 320: 57-58.

Paerl, H. W., and J. Huisman. 2009. Climate change: a catalyst for global expansion of harmful cyanobacterial blooms. Environ. Microbiol. Rep. 1: 27-37.

Palmer, J., S. Flint, and J. Brooks. 2007. Bacterial cell attachment, the beginning of a biofilm. J. Ind. Microbiol. Biotechnol. 34: 577-588.

Pandey, P. K., M. S. Laxmi, and S. Kumar. 2014. In vitro evaluation of natural and synthetic substrate for biofilm formation and their effect on water qualities. Indian J. Anim. Res. 48: 585-592.

Park, R. A., J. S. Clough, and M. C. Wellman. 2008. AQUATOX: Modeling environmental fate and ecological effects in aquatic ecosystems. Ecol. Modell. 213: 1-15. 
Park, S., M. T. Brett, E. T. Oshel, and C. R. Goldman. 2003. Seston food quality and Daphnia production efficiencies in an oligo-mesotrophic subalpine lake. Aquat. Ecol. 37: 123-136.

Parker, P. L., C. van Baalen, and L. Maurer. 1967. Fatty acids in eleven species of blue-green algae: geochemical significance. Science 155: 707-708.

Parker, P. L., and R. F. Leo. 1965. Fatty acids in blue-green algal mat communities. Science 148: 373-374.

Parmesan, C., and G. Yohe. 2003. A globally coherent fingerprint of climate change impacts across natural systems. Nature 421: 37-42.

Parrish, C. C. 2009. Essential fatty acids in aquatic food webs, p. 309-326. In M. T. Arts, M. T. Brett, and M. Kainz [eds.], Lipids in aquatic ecosystems. Springer.

Pasquet, V., L. Ulmann, V. Mimouni, F. Guihéneuf, B. Jacquette, A. Morant-Manceau, and G. Tremblin. 2014. Fatty acids profile and temperature in the cultured marine diatom Odontella aurita. J. Appl. Phycol. 26: 2265-2271.

Pastorella, G., G. Gazzola, S. Guadarrama, and E. Marsili. 2012. Biofilms: applications in bioremediation, p. 73-98. In G. Lear and G. D. Lewis [eds.], Microbial biofilms: Current research and applications. Caister Academic Press.

Patil, V., T. Källqvist, E. Olsen, G. Vogt, and H. R. Gislerød. 2007. Fatty acid composition of 12 microalgae for possible use in aquaculture feed. Aquac. Int. 15: 1-9.

Patrick, D. A., N. Boudreau, Z. Bozic, and others. 2012. Effects of climate change on late-season growth and survival of native and non-native species of watermilfoil (Myriophyllum spp.): implications for invasive potential and ecosystem change. Aquat. Bot. 103: 83-88.

Patrick, R. 1971. The effects of increasing light and temperature on the structure of diatom communities. Limnol. Oceanogr. 16: 405-421.

Patrick, R., B. Crum, and J. Coles. 1969. Temperature and manganese as determining factors in the presence of diatom or blue-green algal floras in streams. Proc. Natl. Acad. Sci. 64: 472478.

Paul, B. J., H. C. Duthie, and W. D. Taylor. 1991. Nutrient cycling by biofilms in running waters of differing nutrient status. J. North Am. Benthol. Soc. 10: 31-41.

Paul, C., B. Matthiessen, and U. Sommer. 2015. Warming, but not enhanced $\mathrm{CO}_{2}$ concentration, quantitatively and qualitatively affects phytoplankton biomass. Mar. Ecol. Prog. Ser. 528: 39-51.

Paul, V. J. 2008. Global warming and cyanobacterial harmful algal blooms, p. 239-257. In H. K. Hudnell [ed.], Cyanobacterial harmful algal blooms: State of the science and research needs. Springer.

Peltomaa, E., M. D. Johnson, and S. J. Taipale. 2017. Marine cryptophytes are great sources of EPA and DHA. Mar. Drugs 16: 3.

Persson, J., and T. Vrede. 2006. Polyunsaturated fatty acids in zooplankton: variation due to taxonomy and trophic position. Freshw. Biol. 51: 887-900.

Peters, L., C. Faust, and W. Traunspurger. 2012. Changes in community composition, carbon and nitrogen stable isotope signatures and feeding strategy in epilithic aquatic nematodes along a depth gradient. Aquat. Ecol. 46: 371-384.

Petersen, J. E., J. C. Cornwell, and W. M. Kemp. 1999. Implicit scaling in the design of experimental aquatic ecosystems. Oikos 85: 3-18.

Peterson, B. J., L. Deegan, J. Helfrich, and others. 1993. Biological responses of a tundra river to fertilization. Ecology 74: 653-672. 
Peterson, B. J., J. E. Hobbie, A. E. Hershey, and others. 1985. Transformation of a tundra river from heterotrophy to autotrophy by addition of phosphorus. Science 229: 1383-1386.

Peterson, C. G., and N. B. Grimm. 1992. Temporal variation in enrichment effects during periphyton succession in a nitrogen-limited desert stream ecosystem. J. North Am. Benthol. Soc. 11: 20-36.

Phillips, G. L., D. Eminson, and B. Moss. 1978. A mechanism to account for macrophyte decline in progressively eutrophicated freshwaters. Aquat. Bot. 4: 103-126.

Phinney, H. K., and C. D. McIntire. 1965. Effect of temperature on metabolism of periphyton communities developed in laboratory streams. Limnol. Oceanogr. 10: 341-345.

Piepho, M., D. Martin-Creuzburg, and A. Wacker. 2012. Phytoplankton sterol contents vary with temperature, phosphorus and silicate supply: a study on three freshwater species. Eur. J. Phycol. 47: 138-145.

Ploug, H., C. Lassen, and B. B. Järgensen. 1993. Action spectra of microalgal photosynthesis and depth distribution of spectral scalar irradiance in a coastal marine sediment of Limfjorden, Denmark. FEMS Microbiol. Ecol. 11: 261-270.

Popova, O. N., A. Y. Haritonov, N. N. Sushchik, O. N. Makhutova, G. S. Kalachova, A. A. Kolmakova, and M. I. Gladyshev. 2017. Export of aquatic productivity, including highly unsaturated fatty acids, to terrestrial ecosystems via Odonata. Sci. Total Environ. 581: 4048.

Powles, S. B. 1984. Photoinhibition of photosynthesis induced by visible light. Annu. Rev. Plant Physiol. 35: 15-44.

Pringle, C. M. 1987. Effects of water and substratum nutrient supplies on lotic periphyton growth: an integrated bioassay. Can. J. Fish. Aquat. Sci. 44: 619-629.

Pringle, C. M., and J. A. Bowers. 1984. An in situ substratum fertilization technique: diatom colonization on nutrient-enriched, sand substrata. Can. J. Fish. Aquat. Sci. 41: 1247-1251.

Proia, L., A. M. Romaní, and S. Sabater. 2012. Nutrients and light effects on stream biofilms: a combined assessment with CLSM, structural and functional parameters. Hydrobiologia 695: 281-291.

Qin, P., C. M. Mayer, K. L. Schulz, X. Ji, and M. E. Ritchie. 2007. Ecological stoichiometry in benthic food webs: effect of light and nutrients on periphyton food quantity and quality in lakes. Limnol. Oceanogr. 52: 1728-1734.

Quinn, J. M., and C. W. Hickey. 1994. Hydraulic parameters and benthic invertebrate distributions in two gravel-bed New Zealand rivers. Freshw. Biol. 32: 489-500.

R Core Team. 2016. R: A language and environment for statistical computing. R Foundation for Statistical Computing, Vienna, Austria. URL https://www.R-project.org/.

Rader, R. B., and T. A. Belish. 1997. Effects of ambient and enhanced UV-B radiation on periphyton in a mountain stream. J. Freshw. Ecol. 12: 615-628.

Raeder, U., J. Ruzicka, and C. Goos. 2010. Characterization of the light attenuation by periphyton in lakes of different trophic state. Limnol. Manag. Inl. Waters 40: 40-46.

Raghupathi, P. K., W. Liu, K. Houf, K. Sabbe, M. Burmølle, and S. J. Sørensen. 2017. Synergistic interactions within a multispecies biofilm enhance individual species protection against grazing by a pelagic protozoan. Front. Microbiol. 8: 2649.

Rasconi, S., A. Gall, K. Winter, and M. J. Kainz. 2015. Increasing water temperature triggers dominance of small freshwater plankton. PLoS One 10: e0140449. 
Rasconi, S., K. Winter, and M. J. Kainz. 2017. Temperature increase and fluctuation induce phytoplankton biodiversity loss-Evidence from a multi-seasonal mesocosm experiment. Ecol. Evol. 7: 2936-2946.

Rasmussen, B. 2000. Filamentous microfossils in a 3,235-million-year-old volcanogenic massive sulphide deposit. Nature 405: 676-679.

Ravet, J. L., M. T. Brett, and G. B. Arhonditsis. 2010. The effects of seston lipids on zooplankton fatty acid composition in Lake Washington, Washington, USA. Ecology 91: 180-190.

Ravet, J. L., M. T. Brett, and D. C. Müller-Navarra. 2003. A test of the role of polyunsaturated fatty acids in phytoplankton food quality for Daphnia using liposome supplementation. Limnol. Oceanogr. 48: 1938-1947.

Ravet, J. L., J. Persson, and M. T. Brett. 2012. Threshold dietary polyunsaturated fatty acid concentrations for Daphnia pulex growth and reproduction. Inl. Waters 2: 199-209.

Reid, G. K. 1961. Ecology of inland waters and estuaries. Reinhold Publishing Corporation.

Renaud, S. M., L. V. Thinh, G. Lambrinidis, and D. L. Parry. 2002. Effect of temperature on growth, chemical composition and fatty acid composition of tropical Australian microalgae grown in batch cultures. Aquaculture 211: 195-214.

Rendueles, O., C. Beloin, P. Latour-Lambert, and J. M. Ghigo. 2014. A new biofilm-associated colicin with increased efficiency against biofilm bacteria. ISME J. 8: 1275-1288.

Rendueles, O., and J. M. Ghigo. 2015. Mechanisms of competition in biofilm communities. Microbiol. spectr 3: 1-18.

Rendueles, O., L. Travier, P. Latour-Lambert, T. Fontaine, J. Magnus, E. Denamur, and J. M. Ghigo. 2011. Screening of Escherichia coli species biodiversity reveals new biofilmassociated antiadhesion polysaccharides. MBio 2: e00043-11.

Reuter, J. E., and R. P. Axler. 1992. Physiological characteristics of inorganic nitrogen uptake by spatially separate algal communities in a nitrogen deficient lake. Freshw. Biol. 27: 227-236.

Reuter, J. E., S. L. Loeb, and C. R. Goldman. 1986. Inorganic nitrogen uptake by epilithic periphyton in a N-deficient lake. Limnol. Oceanogr. 31: 149-160.

Reynolds, C. S., and P. S. Davies. 2001. Sources and bioavailability of phosphorus fractions in freshwaters: a British perspective. Biol. Rev. 76: 27-64.

Rezanka, T., I. Dor, A. Prell, and V. M. Dembitsky. 2003. Fatty acid composition of six freshwater wild cyanobacterial species. Folia Microbiol. 48: 71-75.

Rhee, G., and I. J. Gotham. 1981. The effect of environmental factors on phytoplankton growth: Temperature and the interactions of temperature with nutrient limitation. Limnol. Oceanogr. 26: 635-648.

Richardson, K., J. Beardall, and J. A. Raven. 1983. Adaptation of unicellular algae to irradiance: an analysis of strategies. New Phytol. 93: 157-191.

Richardson, T. L., C. E. Gibson, and S. I. Heaney. 2000. Temperature, growth and seasonal succession of phytoplankton in Lake Baikal, Siberia. Freshw. Biol. 44: 431-440.

Richoux, N. B., L. Bergamino, S. Moyo, and T. Dalu. 2018. Spatial and temporal variability in the nutritional quality of basal resources along a temperate river/estuary continuum. Org. Geochem. 116: 1-12.

Rier, S. T., and R. J. Stevenson. 2001. Relation of environmental factors to density of epilithic lotic bacteria in 2 ecoregions. J. North Am. Benthol. Soc. 20: 520-532. 
Rier, S. T., and R. J. Stevenson. 2002. Effects of light, dissolved organic carbon, and inorganic nutrients [2pt] on the relationship between algae and heterotrophic bacteria in stream periphyton. Hydrobiologia 489: 179-184.

del Rio, C. M., and S. R. McWilliams. 2016. How essential fats affect bird performance and link aquatic ecosystems and terrestrial consumers. Proc. Natl. Acad. Sci. 113: 11988-11990.

Risse-Buhl, U., M. Karsubke, J. Schlief, C. Baschien, M. Weitere, and M. Mutz. 2012. Aquatic protists modulate the microbial activity associated with mineral surfaces and leaf litter. Aquat. Microb. Ecol. 66: 133-147.

Robarts, R. D., and T. Zohary. 1987. Temperature effects on photosynthetic capacity, respiration, and growth rates of bloom-forming cyanobacteria. New Zeal. J. Mar. Freshw. Res. 21: 391399.

Roberts, S., S. Sabater, and J. Beardall. 2004. Benthic microalgal colonization in streams of differing riparian cover and light availability. J. Phycol. 40: 1004-1012.

Roeder, D. R. 1977. Relationships between phytoplankton and periphyton communities in a central Iowa stream. Hydrobiologia 56: 145-151.

Romaní, A. M. 2010. Freshwater biofilms, p. 137-153. In S. Dürr and J. C. Thomason [eds.], Biofouling. Wiley-Blackwell.

Romaní, A. M., and S. Sabater. 1999. Effect of primary producers on the heterotrophic metabolism of a stream biofilm. Freshw. Biol. 41: 729-736.

Rosa, J., V. Ferreira, C. Canhoto, and M. A. S. Graça. 2013. Combined effects of water temperature and nutrients concentration on periphyton respiration-implications of global change. Int. Rev. Hydrobiol. 98: 14-23.

Rosemond, A. D. 1993. Interactions among irradiance, nutrients, and herbivores constrain a stream algal community. Oecologia 94: 585-594.

Rosemond, A. D. 1994. Multiple factors limit seasonal variation in periphyton in a forest stream. J. North Am. Benthol. Soc. 13: 333-344.

Rosemond, A. D., P. J. Mulholland, and S. H. Brawley. 2000. Seasonally shifting limitation of stream periphyton: response of algal populations and assemblage biomass and productivity to variation in light, nutrients, and herbivores. Can. J. Fish. Aquat. Sci. 57: 66-75.

Rosemond, A. D., P. J. Mulholland, and J. W. Elwood. 1993. Top-down and bottom-up control of stream periphyton: effects of nutrients and herbivores. Ecology 74: 1264-1280.

Rouf, A. J. M. A., S. M. Phang, and M. A. Ambak. 2010. Depth distribution and ecological preferences of periphytic algae in Kenyir Lake, the largest tropical reservoir of Malaysia. Chinese J. Oceanol. Limnol. 28: 856-867.

Round, F. E. 1961. Studies on bottom-living algae in some lakes of the English lake district. J. Ecol. 245-254.

Round, F. E. 1984. The ecology of algae. Cambridge University Press.

Round, F. E., and J. W. Eaton. 1966. Persistent, vertical-migration rhythms in benthic microflora: III. The rhythm of epipelic algae in a freshwater pond. J. Ecol. 54: 609-615.

Ruttner, F., D. G. Frey, and F. E. J. Fry. 1953. Fundamentals of limnology. University Of Toronto Press.

Saikia, S. K. 2011. Review on periphyton as mediator of nutrient transfer in aquatic ecosystems. Ecol. Balk. 3: 65-78. 
Salama, Y., M. Chennaoui, A. Sylla, M. Mountadar, M. Rihani, and O. Assobhei. 2016. Characterization, structure, and function of extracellular polymeric substances (EPS) of microbial biofilm in biological wastewater treatment systems: a review. Desalin. Water Treat. 57: 16220-16237.

Sallal, A. K., N. A. Nimer, and S. S. Radwan. 1990. Lipid and fatty acid composition of freshwater cyanobacteria. Microbiology 136: 2043-2048.

Sanches, L. F., R. D. Guariento, A. Caliman, R. L. Bozelli, and F. A. Esteves. 2011. Effects of nutrients and light on periphytic biomass and nutrient stoichiometry in a tropical blackwater aquatic ecosystem. Hydrobiologia 669: 35-44.

Sánchez, M. L., G. L. Pérez, I. Izaguirre, and H. Pizarro. 2013. Influence of underwater light climate on periphyton and phytoplankton communities in shallow lakes from the Pampa plain (Argentina) with contrasting steady states. J. Limnol. 72: 62-78.

Sand-Jensen, K. 1977. Effect of epiphytes on eelgrass photosynthesis. Aquat. Bot. 3: 55-63.

Sand-Jensen, K., and J. Borum. 1984. Epiphyte shading and its effect on photosynthesis and diel metabolism of Lobelia dortmanna L. during the spring bloom in a Danish lake. Aquat. Bot. 20: $109-119$.

Sand-Jensen, K., and J. Borum. 1991. Interactions among phytoplankton, periphyton, and macrophytes in temperate freshwaters and estuaries. Aquat. Bot. 41: 137-175.

Sand-Jensen, K., and N. P. Revsbech. 1987. Photosynthesis and light adaptation in epiphytemacrophyte associations measured by oxygen microelectrodes. Limnol. Oceanogr. 32: 452457.

Sand-Jensen, K., N. P. Revsbech, and B. B. Jörgensen. 1985. Microprofiles of oxygen in epiphyte communities on submerged macrophytes. Mar. Biol. 89: 55-62.

Sand-Jensen, K., and M. Søndergaard. 1981. Phytoplankton and epiphyte development and their shading effect on submerged macrophytes in lakes of different nutrient status. Int. Rev. Hydrobiol. 66: 529-552.

Sanpera-Calbet, I., I. Ylla, A. M. Romaní, S. Sabater, and I. Muñoz. 2017. Drought effects on resource quality in a Mediterranean stream: fatty acids and sterols as indicators. Limnetica 36: $29-43$.

Sargent, J. R., D. R. Tocher, and J. G. Bell. 2003. The Lipids, p. 181-257. In J. E. Halver and R. W. Hardy [eds.], Fish nutrition: third edition. Academic Press.

Schabhüttl, S., P. Hingsamer, G. Weigelhofer, T. Hein, A. Weigert, and M. Striebel. 2013. Temperature and species richness effects in phytoplankton communities. Oecologia 171: 527-536.

Schanz, F. 1985. Vertical light attenuation and phytoplankton development in Lake Zürich. Limnol. Oceanogr. 30: 299-310.

Scheffer, M., S. H. Hosper, M. L. Meijer, B. Moss, and E. Jeppesen. 1993. Alternative equilibria in shallow lakes. Trends Ecol. Evol. 8: 275-279.

Schiefer, G. E., and D. E. Caldwell. 1982. Synergistic interaction between Anabaena and Zoogloea spp. in carbon dioxide-limited continuous cultures. Appl. Environ. Microbiol. 44: 84-87.

Schindler, D. W., R. H. Hesslein, and M. A. Turner. 1987. Exchange of nutrients between sediments and water after 15 years of experimental eutrophication. Can. J. Fish. Aquat. Sci. 44: 26-33.

Schlesinger, D. A., and B. J. Shuter. 1981. Patterns of growth and cell composition of freshwater algae in light-limited continuous cultures. J. Phycol. 17: 250-256. 
Schnurr, P. J., and D. G. Allen. 2015. Factors affecting algae biofilm growth and lipid production: a review. Renew. Sustain. Energy Rev. 52: 418-429.

Schönfeld, P., and L. Wojtczak. 2016. Short-and medium-chain fatty acids in energy metabolism: the cellular perspective. J. Lipid Res. 57: 943-954.

Schroeder, F., D. Kathöfer, W. Traunspurger, and L. Peters. 2013. Grazing effects on nematodes and other components of the periphyton at three depths in Lake Erken. Freshw. Sci. 32: $1088-1100$.

Schulz, R., M. Bundschuh, R. Gergs, and others. 2015. Review on environmental alterations propagating from aquatic to terrestrial ecosystems. Sci. Total Environ. 538: 246-261.

Scott, J. T., J. A. Back, J. M. Taylor, and R. S. King. 2008. Does nutrient enrichment decouple algal-bacterial production in periphyton? J. North Am. Benthol. Soc. 27: 332-344.

Scott, J. T., and R. D. Doyle. 2006. Coupled photosynthesis and heterotrophic bacterial biomass production in a nutrient-limited wetland periphyton mat. Aquat. Microb. Ecol. 45: 69-77.

Scrimgeour, G. J., J. M. Culp, M. L. Bothwell, F. J. Wrona, and M. H. McKee. 1991. Mechanisms of algal patch depletion: importance of consumptive and non-consumptive losses in mayfly-diatom systems. Oecologia 85: 343-348.

Seaburg, K. G., and B. C. Parker. 1983. Seasonal differences in the temperature ranges of growth of Virginia algae. J. Phycol. 19: 380-386.

Seekell, D. A., J. F. Lapierre, and J. Karlsson. 2015. Trade-offs between light and nutrient availability across gradients of dissolved organic carbon concentration in Swedish lakes: implications for patterns in primary production. Can. J. Fish. Aquat. Sci. 72: 1663-1671.

Sekar, R., K. V. K. Nair, V. N. R. Rao, and V. P. Venugopalan. 2002. Nutrient dynamics and successional changes in a lentic freshwater biofilm. Freshw. Biol. 47: 1893-1907.

Sekar, R., V. P. Venugopalan, K. Nandakumar, K. V. K. Nair, and V. N. R. Rao. 2004. Early stages of biofilm succession in a lentic freshwater environment, p. 97-108. In P. O. Ang Jr. [ed.], Asian Pacific phycology in the $21^{\text {st }}$ century: prospects and challenges. Springer.

Seligo, A. 1905. Über den Ursprung der Fischnahrung. Mitt. d. Westpr. Fisch 17: 52-56.

Serôdio, J., J. Marques da Silva, and F. Catarino. 1997. Nondestructive tracing of migratory rhythms of intertidal benthic microalgae using in vivo chlorophyll a fluorescence. J. Phycol. 33: 542-553.

Shapiro, S. S., and M. B. Wilk. 1965. An analysis of variance test for normality (complete samples). Biometrika 52: 591-611.

Sharma, K., P. W. Inglett, K. R. Reddy, and A. V Ogram. 2005. Microscopic examination of photoautotrophic and phosphatase-producing organisms in phosphorus-limited Everglades periphyton mats. Limnol. Oceanogr. 50: 2057-2062.

Sheath, R. G., J. M. Burkholder, M. O. Morison, A. D. Steinman, and K. L. VanAlstyne. 1986. Effect of tree canopy removal by gypsy moth larvae on the macroalgae of a Rhode Island headwater stream. J. Phycol. 22: 567-570.

Shikata, T., and S. Shimeno. 1994. Metabolic response to dietary stearic acid, linoleic acid, and highly unsaturated fatty acid in carp. Fish. Sci. 60: 735-739.

Shimeno, S., H. Hosokawa, and M. Takeda. 1996. Metabolic response of juvenile yellowtail to dietary carbohydrate to lipid ratios. Fish. Sci. 62: 945-949.

Shimeno, S., D. Kheyyali, and T. Shikata. 1995. Metabolic response to dietary lipid to protein ratios in common carp. Fish. Sci. 61: 977-980. 
Shurin, J. B., J. L. Clasen, H. S. Greig, P. Kratina, and P. L. Thompson. 2012. Warming shifts top-down and bottom-up control of pond food web structure and function. Philos. Trans. R. Soc. B Biol. Sci. 367: 3008-3017.

Sievert, S. M., and C. Vetriani. 2012. Chemoautotrophy at deep-sea vents: past, present, and future. Oceanography 25: 218-233.

Simon, K. S., E. F. Benfield, and S. A. Macko. 2003. Food web structure and the role of epilithic biofilms in cave streams. Ecology 84: 2395-2406.

Sinensky, M. 1974. Homeoviscous adaptation-a homeostatic process that regulates the viscosity of membrane lipids in Escherichia coli. Proc. Natl. Acad. Sci. 71: 522-525.

Singh, R., D. Paul, and R. K. Jain. 2006. Biofilms: implications in bioremediation. Trends Microbiol. 14: 389-397.

Smyntek, P. M., M. A. Teece, K. L. Schulz, and A. J. Storch. 2008. Taxonomic differences in the essential fatty acid composition of groups of freshwater zooplankton relate to reproductive demands and generation time. Freshw. Biol. 53: 1768-1782.

Sommer, U. 1996. Nutrient competition experiments with periphyton from the Baltic Sea. Mar. Ecol. Prog. Ser. 140: 161-167.

Sommer, U., and K. Lengfellner. 2008. Climate change and the timing, magnitude, and composition of the phytoplankton spring bloom. Glob. Chang. Biol. 14: 1199-1208.

Song, B., and L. G. Leff. 2006. Influence of magnesium ions on biofilm formation by Pseudomonas fluorescens. Microbiol. Res. 161: 355-361.

Song, K., M. A. Xenopoulos, J. M. Buttle, J. Marsalek, N. D. Wagner, F. R. Pick, and P. C. Frost. 2013. Thermal stratification patterns in urban ponds and their relationships with vertical nutrient gradients. J. Environ. Manage. 127: 317-323.

Souffreau, C., P. Vanormelingen, B. van de Vijver, T. Isheva, E. Verleyen, K. Sabbe, and W. Vyverman. 2013. Molecular evidence for distinct Antarctic lineages in the cosmopolitan terrestrial diatoms Pinnularia borealis and Hantzschia amphioxys. Protist 164: 101-115.

Squires, L. E., S. R. Rushforth, and J. D. Brotherson. 1979. Algal response to a thermal effluent: study of a power station on the Provo River, Utah, USA. Hydrobiologia 63: 17-32.

Stalder, T., and E. Top. 2016. Plasmid transfer in biofilms: a perspective on limitations and opportunities. NPJ Biofilms Microbiomes 2: 16022.

Stanley-Samuelson, D. W., R. A. Jurenka, C. Cripps, G. J. Blomquist, and M. de Renobales. 1988. Fatty acids in insects: composition, metabolism, and biological significance. Arch. Insect Biochem. Physiol. 9: 1-33.

Stanley, D. W., and R. J. Daley. 1976. Environmental control of primary productivity in Alaskan tundra ponds. Ecology 57: 1025-1033.

Stanley, E. H., R. A. Short, J. W. Harrison, R. Hall, and R. C. Wiedenfeld. 1990. Variation in nutrient limitation of lotic and lentic algal communities in a Texas (USA) river. Hydrobiologia 206: 61-71.

Steinman, A. D. 1992. Does an increase in irradiance influence periphyton in a heavily-grazed woodland stream? Oecologia 91: 163-170.

Steinman, A. D. 1996. Effects of grazers on freshwater benthic algae, p. 341-373. In R. J. Stevenson, M. L. Bothwell, and R. L. Lowe [eds.], Aquatic ecology. Academic Press.

Steinman, A. D., and C. D. McIntire. 1987. Effects of irradiance on the community structure and biomass of algal assemblages in laboratory streams. Can. J. Fish. Aquat. Sci. 44: 16401648. 
Steinman, A. D., R. H. Meeker, A. J. Rodusky, W. P. Davis, and S. J. Hwang. 1997. Ecological properties of charophytes in a large subtropical lake. J. North Am. Benthol. Soc. 16: 781793.

Stelzer, R. S., and G. A. Lamberti. 2001. Effects of N: P ratio and total nutrient concentration on stream periphyton community structure, biomass, and elemental composition. Limnol. Oceanogr. 46: 356-367.

Sterner, R. W., J. J. Elser, E. J. Fee, S. J. Guildford, and T. H. Chrzanowski. 1997. The light: nutrient ratio in lakes: the balance of energy and materials affects ecosystem structure and process. Am. Nat. 150: 663-684.

Stevenson, R. J., R. Singer, D. A. Roberts, and C. W. Boylen. 1985. Patterns of epipelic algal abundance with depth, trophic status, and acidity in poorly buffered New Hampshire lakes. Can. J. Fish. Aquat. Sci. 42: 1501-1512.

Stevenson, R. J., and E. F. Stoermer. 1981. Quantitative differences between benthic algal communities along a depth gradient in Lake Michigan. J. Phycol. 17: 29-36.

Stewart, R. I. A., M. Dossena, D. A. Bohan, and others. 2013. Mesocosm experiments as a tool for ecological climate-change research. Adv. Ecol. Res. 48: 71-181.

Stockner, J. G., and K. R. S. Shortreed. 1978. Enhancement of autotrophic production by nutrient addition in a coastal rainforest stream on Vancouver Island. J. Fish. Board Canada 35: 2834.

Støttrup, J. G., and J. Jensen. 1990. Influence of algal diet on feeding and egg-production of the calanoid copepod Acartia tonsa Dana. J. Exp. Mar. Bio. Ecol. 141: 87-105.

Strandberg, U., S. J. Taipale, M. Hiltunen, A. W. E. Galloway, M. T. Brett, and P. Kankaala. 2015. Inferring phytoplankton community composition with a fatty acid mixing model. Ecosphere 6: 1-18.

Strecker, A. L., T. P. Cobb, and R. D. Vinebrooke. 2004. Effects of experimental greenhouse warming on phytoplankton and zooplankton communities in fishless alpine ponds. Limnol. Oceanogr. 49: 1182-1190.

Sumner, W. T., and S. G. Fisher. 1979. Periphyton production in Fort River, Massachusetts. Freshw. Biol. 9: 205-212.

Sundbäck, K., and B. Jönsson. 1988. Microphytobenthic productivity and biomass in sublittoral sediments of a stratified bay, southeastern Kattegat. J. Exp. Mar. Bio. Ecol. 122: 63-81.

Sushchik, N. N., M. I. Gladyshev, E. A. Ivanova, and E. S. Kravchuk. 2010. Seasonal distribution and fatty acid composition of littoral microalgae in the Yenisei River. J. Appl. Phycol. 22: 11-24.

Sushchik, N. N., M. I. Gladyshev, O. N. Makhutova, G. S. Kalachova, E. S. Kravchuk, and E. A. Ivanova. 2004. Associating particulate essential fatty acids of the $\omega 3$ family with phytoplankton species composition in a Siberian reservoir. Freshw. Biol. 49: 1206-1219.

Sushchik, N. N., M. I. Gladyshev, A. V Moskvichova, O. N. Makhutova, and G. S. Kalachova. 2003a. Comparison of fatty acid composition in major lipid classes of the dominant benthic invertebrates of the Yenisei River. Comp. Biochem. Physiol. Part B Biochem. Mol. Biol. 134: $111-122$.

Sushchik, N. N., G. S. Kalachova, N. O. Zhila, M. I. Gladyshev, and T. G. Volova. 2003b. A temperature dependence of the intra-and extracellular fatty-acid composition of green algae and cyanobacterium. Russ. J. plant Physiol. 50: 374-380. 
Sushchik, N. N., Y. A. Yurchenko, M. I. Gladyshev, O. E. Belevich, G. S. Kalachova, and A. A. Kolmakova. 2013. Comparison of fatty acid contents and composition in major lipid classes of larvae and adults of mosquitoes (Diptera: Culicidae) from a steppe region. Insect Sci. 20: 585-600.

Taipale, S. J., U. Strandberg, E. Peltomaa, A. W. E. Galloway, A. Ojala, and M. T. Brett. 2013. Fatty acid composition as biomarkers of freshwater microalgae: analysis of 37 strains of microalgae in 22 genera and in seven classes. Aquat. Microb. Ecol. 71: 165-178.

Tarkowska-Kukuryk, M., and T. Mieczan. 2012. Effect of substrate on periphyton communities and relationships among food web components in shallow hypertrophic lake. J. Limnol. 71: 279-290.

Taulbee, W. K., S. D. Cooper, and J. M. Melack. 2005. Effects of nutrient enrichment on algal biomass across a natural light gradient. Arch. für Hydrobiol. 164: 449-464.

Teitzel, G. M., and M. R. Parsek. 2003. Heavy metal resistance of biofilm and planktonic Pseudomonas aeruginosa. Appl. Environ. Microbiol. 69: 2313-2320.

Tekwani, N., N. Majdi, B. Mialet, E. Tornès, G. Urrea-Clos, E. Buffan-Dubau, S. Sabater, and M. Tackx. 2013. Contribution of epilithic diatoms to benthic-pelagic coupling in a temperate river. Aquat. Microb. Ecol. 69: 47-57.

Teoh, M. L., W. L. Chu, H. Marchant, and S. M. Phang. 2004. Influence of culture temperature on the growth, biochemical composition and fatty acid profiles of six Antarctic microalgae. J. Appl. Phycol. 16: 421-430.

Teoh, M. L., S. M. Phang, and W. L. Chu. 2013. Response of Antarctic, temperate, and tropical microalgae to temperature stress. J. Appl. Phycol. 25: 285-297.

Terlizzi, A., and M. Faimali. 2010. Fouling on artificial substrata, p. 170-184. In S. Dürr and J. C. Thomason [eds.], Biofouling. Wiley-Blackwell.

Thomas, D. N., and G. S. Dieckmann. 2002. Antarctic sea ice--a habitat for extremophiles. Science 295: 641-644.

Thomen, P., J. Robert, A. Monmeyran, A. F. Bitbol, C. Douarche, and N. Henry. 2017. Bacterial biofilm under flow: First a physical struggle to stay, then a matter of breathing. PLoS One 12: e0175197.

Thompson, P. A., M. Guo, and P. J. Harrison. 1992a. Effects of variation in temperature. I. On the biochemical composition of eight species of marine phytoplankton. J. Phycol. 28: 481488.

Thompson, P. A., M. Guo, and P. J. Harrison. 1993. The influence of irradiance on the biochemical composition of three phytoplankton species and their nutritional value for larvae of the Pacific oyster (Crassostrea gigas). Mar. Biol. 117: 259-268.

Thompson, P. A., M. Guo, P. J. Harrison, and J. N. C. Whyte. 1992b. Effects of variation in temperature. II. On the fatty acid composition of eight species of marine phytoplankton. J. Phycol. 28: 488-497.

Thompson, P. A., P. J. Harrison, and J. N. C. Whyte. 1990. Influence of irradiance on the fatty acid composition of phytoplankton. J. Phycol. 26: 278-288.

Thorp, J. H., and M. R. Diggins. 1982. Factors affecting depth distribution of dragonflies and other benthic insects in a thermally destabilized reservoir. Hydrobiologia 87: 33-44.

Timke, M., N. Q. Wang-Lieu, K. Altendorf, and A. Lipski. 2005. Fatty acid analysis and spoilage potential of biofilms from two breweries. J. Appl. Microbiol. 99: 1108-1122.

Tison, D. L., and A. J. Lingg. 1979. Dissolved organic matter utilization and oxygen uptake in algal-bacterial microcosms. Can. J. Microbiol. 25: 1315-1320. 
Tocher, D. R. 2003. Metabolism and functions of lipids and fatty acids in teleost fish. Rev. Fish. Sci. 11: 107-184.

Tocher, D. R. 2010. Fatty acid requirements in ontogeny of marine and freshwater fish. Aquac. Res. 41: 717-732.

Torres-Ruiz, M., J. D. Wehr, and A. A. Perrone. 2007. Trophic relations in a stream food web: importance of fatty acids for macroinvertebrate consumers. J. North Am. Benthol. Soc. 26: 509-522.

Torres-Ruiz, M., J. D. Wehr, and A. A. Perrone. 2010. Are net-spinning caddisflies what they eat? An investigation using controlled diets and fatty acids. J. North Am. Benthol. Soc. 29: 803-813.

Torstensson, A., M. Hedblom, J. Andersson, M. X. Andersson, and A. Wulff. 2013. Synergism between elevated $\mathrm{pCO}_{2}$ and temperature on the Antarctic sea ice diatom Nitzschia lecointei. Biogeosciences 10: 6391-6401.

Toseland, A., S. J. Daines, J. R. Clark, and others. 2013. The impact of temperature on marine phytoplankton resource allocation and metabolism. Nat. Clim. Chang. 3: 979-984.

Tuchman, N. C., and R. J. Stevenson. 1991. Effects of selective grazing by snails on benthic algal succession. J. North Am. Benthol. Soc. 10: 430-443.

Twining, C. W., J. T. Brenna, P. Lawrence, J. R. Shipley, T. N. Tollefson, and D. W. Winkler. 2016. Omega-3 long-chain polyunsaturated fatty acids support aerial insectivore performance more than food quantity. Proc. Natl. Acad. Sci. 113: 10920-10925.

Tyndall, J. 1861. The bakerian lecture.- on the absorption and radiation of heat by gases and vapours, and on the physical connexion of radiation, absorption, and conduction. Philos. Trans. R. Soc. London 151: 1-36.

Ullyott, P., and P. Holmes. 1936. Thermal stratification in lakes. Nature 138: 971.

Underwood, G. J. C., R. G. Perkins, M. C. Consalvey, A. R. M. Hanlon, K. Oxborough, N. R. Baker, and D. M. Paterson. 2005. Patterns in microphytobenthic primary productivity: Species-specific variation in migratory rhythms and photosynthetic efficiency in mixedspecies biofilms. Limnol. Oceanogr. 50: 755-767.

US EPA. 1996. Method 1669: Sampling ambient water for trace metals at EPA water quality criteria levels. US Environmental Protection Agency. Office of Water, Washington, DC.

Vadeboncoeur, Y., and D. M. Lodge. 2000. Periphyton production on wood and sediment: substratum-specific response to laboratory and whole-lake nutrient manipulations. J. North Am. Benthol. Soc. 19: 68-81.

Vadeboncoeur, Y., D. M. Lodge, and S. R. Carpenter. 2001. Whole-lake fertilization effects on distribution of primary production between benthic and pelagic habitats. Ecology 82: 10651077.

Vadeboncoeur, Y., and A. D. Steinman. 2002. Periphyton function in lake ecosystems. Sci. World J. 2: 1449-1468.

Verreth, J., J. Coppoolse, and H. Segner. 1994. The effect of low HUFA-and high HUFAenriched Artemia, fed at different feeding levels, on growth, survival, tissue fatty acids and liver histology of Clarias gariepinus larvae. Aquaculture 126: 137-150.

Videla, H. A., and L. K. Herrera. 2005. Microbiologically influenced corrosion: looking to the future. Int. Microbiol. 8: 169.

Vincent, W. F., R. W. Castenholz, M. T. Downes, and C. Howard-Williams. 1993. Antarctic cyanobacteria: light, nutrients, and photosynthesis in the microbial mat environment. J. Phycol. 29: 745-755. 
Vincent, W. F., and C. Howard-Williams. 1986. Antarctic stream ecosystems: physiological ecology of a blue-green algal epilithon. Freshw. Biol. 16: 219-233.

Vincent, W. F., and S. Roy. 1993. Solar ultraviolet-B radiation and aquatic primary production: damage, protection, and recovery. Environ. Rev. 1: 1-12.

Vinebrooke, R. D., and P. R. Leavitt. 1996. Effects of ultraviolet radiation on periphyton in an alpine lake. Limnol. Oceanogr. 41: 1035-1040.

Vinebrooke, R. D., and P. R. Leavitt. 1999. Differential responses of littoral communities to ultraviolet radiation in an alpine lake. Ecology 80: 223-237.

Vinson, D. K., and S. R. Rushforth. 1989. Diatom species composition along a thermal gradient in the Portneuf River, Idaho, USA. Hydrobiologia 185: 41-54.

Viso, A. C., and J. C. Marty. 1993. Fatty acids from 28 marine microalgae. Phytochemistry 34: $1521-1533$.

Volkman, J. K., S. W. Jeffrey, P. D. Nichols, G. I. Rogers, and C. D. Garland. 1989. Fatty acid and lipid composition of 10 species of microalgae used in mariculture. J. Exp. Mar. Bio. Ecol. 128: 219-240.

Vu, B., M. Chen, R. J. Crawford, and E. P. Ivanova. 2009. Bacterial extracellular polysaccharides involved in biofilm formation. Molecules 14: 2535-2554.

Wacker, A. 2005. Lipids in the food of a terrestrial snail. Invertebr. Reprod. Dev. 47: 205-212.

Wacker, A., and D. Martin-Creuzburg. 2007. Allocation of essential lipids in Daphnia magna during exposure to poor food quality. Funct. Ecol. 21: 738-747.

Wacker, A., M. Piepho, J. L. Harwood, I. A. Guschina, and M. T. Arts. 2016. Light-induced changes in fatty acid profiles of specific lipid classes in several freshwater phytoplankton species. Front. Plant Sci. 7: 264.

Wagner, N. D., A. J. Simpson, and M. J. Simpson. 2018. Sub-Lethal metabolic responses to contaminant mixture toxicity in Daphnia magna. Environ. Toxicol. Chem. 9999: 1-10, doi:10.1002/etc.4208

Wang, Y., D. S. Lin, L. Bolewicz, and W. E. Connor. 2006. The predominance of polyunsaturated fatty acids in the butterfly Morpho peleides before and after metamorphosis. J. Lipid Res. 47: 530-536.

Warren, D. R., W. S. Keeton, H. A. Bechtold, and E. J. Rosi-Marshall. 2013. Comparing streambed light availability and canopy cover in streams with old-growth versus earlymature riparian forests in western Oregon. Aquat. Sci. 75: 547-558.

Watanabe, T. 1982. Lipid nutrition in fish. Comp. Biochem. Physiol. Part B Comp. Biochem. 73: $3-15$.

Watermann, F., H. Hillebrand, G. Gerdes, W. E. Krumbein, and U. Sommer. 1999. Competition between benthic cyanobacteria and diatoms as influenced by different grain sizes and temperatures. Mar. Ecol. Prog. Ser. 187: 77-87.

Watson, S. B., E. McCauley, and J. A. Downing. 1997. Patterns in phytoplankton taxonomic composition across temperate lakes of differing nutrient status. Limnol. Oceanogr. 42: 487495.

Weatherhead, M., and M. R. James. 2001. Distribution of macroinvertebrates in relation to physical and biological variables in the littoral zone of nine New Zealand lakes. Hydrobiologia 462: 115-129.

Weaver, V. B., and R. Kolter. 2004. Burkholderia spp. alter Pseudomonas aeruginosa physiology through iron sequestration. J. Bacteriol. 186: 2376-2384. 
Weers, P., and R. Gulati. 1997. Effect of the addition of polyunsaturated fatty acids to the diet on the growth and fecundity of Daphnia galeata. Freshw. Biol. 38: 721-729.

Weers, P., K. Siewertsen, and R. Gulati. 1997. Is the fatty acid composition of Daphnia galeata determined by the fatty acid composition of the ingested diet? Freshw. Biol. 38: 731-738.

Wehr, J. D. 1981. Analysis of seasonal succession of attached algae in a mountain stream, the North Alouette River, British Columbia. Can. J. Bot. 59: 1465-1474.

Welch, E. B., J. M. Jacoby, R. R. Horner, and M. R. Seeley. 1988. Nuisance biomass levels of periphytic algae in streams. Hydrobiologia 157: 161-168.

Wellnitz, T. A., R. B. Rader, and J. V Ward. 1996. Importance of light and nutrients in structuring an algal community in a Rocky Mountain stream. J. Freshw. Ecol. 11: 399-413.

Westall, F., M. J. de Wit, J. Dann, S. van der Gaast, C. E. J. de Ronde, and D. Gerneke. 2001. Early Archean fossil bacteria and biofilms in hydrothermally-influenced sediments from the Barberton greenstone belt, South Africa. Precambrian Res. 106: 93-116.

Wetzel, R. G. 1964. A comparative study of the primary production of higher aquatic plants, periphyton, and phytoplankton in a large, shallow lake. Int. Rev. Hydrobiol. 49: 1-61.

Wetzel, R. G. 1983. Attached algal-substrata interactions: fact or myth, and when and how?, p. 207-215. In R. G. Wetzel [ed.], Periphyton of freshwater ecosystems. Springer.

Wetzel, R. G. 1993. Microcommunities and microgradients: linking nutrient regeneration, microbial mutualism, and high sustained aquatic primary production. Netherl. J. Aquat. Ecol. 27: 3-9.

Wetzel, R. G. 2001. Limnology: lake and river ecosystems, $3^{\text {rd }}$ ed. Academic Press.

Wilde, E. W. 1982. Responses of attached algal communities to termination of thermal pollution. Hydrobiologia 94: 135-138.

Wilde, E. W., and L. J. Tilly. 1981. Structural characteristics of algal communities in thermally altered artificial streams. Hydrobiologia 76: 57-63.

Wilhelm, L., K. Besemer, L. Fragner, H. Peter, W. Weckwerth, and T. J. Battin. 2015. Altitudinal patterns of diversity and functional traits of metabolically active microorganisms in stream biofilms. ISME J. 9: 2454.

Wilhelm, L., G. A. Singer, C. Fasching, T. J. Battin, and K. Besemer. 2013. Microbial biodiversity in glacier-fed streams. ISME J. 7: 1651.

Willer-Königsberg, A. 1923. Der Aufwuchs der Unterwasserpflanzen: Mit 4 Textfiguren. Int. Vereinigung für Theor. und Angew. Limnol. Verhandlungen 1: 37-57.

Wilson, S. K., D. R. Bellwood, J. H. Choat, and M. J. Furnas. 2003. Detritus in the epilithic algal matrix and its use by coral reef fishes. Oceanogr. Mar. Biol. 41: 279-310.

Winder, M., and D. E. Schindler. 2004. Climate change uncouples trophic interactions in an aquatic ecosystem. Ecology 85: 2100-2106.

Wingender, J., and H. C. Flemming. 2011. Biofilms in drinking water and their role as reservoir for pathogens. Int. J. Hyg. Environ. Health 214: 417-423.

Wingender, J., T. R. Neu, and H. C. Flemming. 1999. What are bacterial extracellular polymeric substances?, p. 1-19. In J. Wingender, T. R. Neu, and H. C. Flemming [eds.], Microbial extracellular polymeric substances. Springer.

Winterbourn, M. J. 1990. Interactions among nutrients, algae and invertebrates in a New Zealand mountain stream. Freshw. Biol. 23: 463-474.

Wissinger, S. A. 1988. Spatial distribution, life history and estimates of survivorship in a fourteen-species assemblage of larval dragonflies (Odonata: Anisoptera). Freshw. Biol. 20: 329-340. 
Wolfstein, K., and L. J. Stal. 2002. Production of extracellular polymeric substances (EPS) by benthic diatoms: effect of irradiance and temperature. Mar. Ecol. Prog. Ser. 236: 13-22.

Woodruff, S. L., W. A. House, M. E. Callow, and B. S. C. Leadbeater. 1999. The effects of biofilms on chemical processes in surficial sediments. Freshw. Biol. 41: 73-89.

Woodward, G. 2009. Biodiversity, ecosystem functioning and food webs in fresh waters: assembling the jigsaw puzzle. Freshw. Biol. 54: 2171-2187.

Woodward, G., D. M. Perkins, and L. E. Brown. 2010. Climate change and freshwater ecosystems: impacts across multiple levels of organization. Philos. Trans. R. Soc. B Biol. Sci. 365: 2093-2106.

Wotton, R. S. 2004. The utiquity and many roles of exopolymers (EPS) in aquatic systems. Sci. Mar. 68: 13-21.

Wright, D. R., and R. D. Hamilton. 1982. Release of methyl mercury from sediments: effects of mercury concentration, low temperature, and nutrient addition. Can. J. Fish. Aquat. Sci. 39: 1459-1466.

Wu, Y., S. Zhang, H. Zhao, and L. Yang. 2010. Environmentally benign periphyton bioreactors for controlling cyanobacterial growth. Bioresour. Technol. 101: 9681-9687.

Wuhrmann, K., and E. Eichenberger. 1975. Experiments on the effects of inorganic enrichment of rivers on periphyton primary production. Int. Vereinigung für Theor. und Angew. Limnol. Verhandlungen 19: 2028-2034.

Wyatt, K. H., and M. R. Turetsky. 2015. Algae alleviate carbon limitation of heterotrophic bacteria in a boreal peatland. J. Ecol. 103: 1165-1171.

Xu, H., H. W. Paerl, B. Qin, G. Zhu, and G. Gaoa. 2010. Nitrogen and phosphorus inputs control phytoplankton growth in eutrophic Lake Taihu, China. Limnol. Oceanogr. 55: 420-432.

Yamamoto, K., S. Haruta, S. Kato, M. Ishii, and Y. Igarashi. 2010. Determinative factors of competitive advantage between aerobic bacteria for niches at the air-liquid interface. Microbes Environ. 25: 317-320.

Yan, L., K. G. Boyd, D. R. Adams, and J. G. Burgess. 2003. Biofilm-specific cross-species induction of antimicrobial compounds in bacilli. Appl. Environ. Microbiol. 69: 3719-3727.

Yang, H., and R. J. Flower. 2012. Effects of light and substrate on the benthic diatoms in an oligotrophic lake: a comparison between natural and artificial substrates. J. Phycol. 48: 1166-1177.

Yang, J. R., and H. C. Duthie. 1995. Regression and weighted averaging models relating surficial sedimentary diatom assemblages to water depth in Lake Ontario. J. Great Lakes Res. 21: 84-94.

Yoder, J. A. 1979. Effect of temperature on light-limited growth and chemical composition of Skeletonema costatum (Bacillariophyceae). J. Phycol. 15: 362-370.

Yoshii, K. 1999. Stable isotope analyses of benthic organisms in Lake Baikal. Hydrobiologia 411: $145-159$.

Young, O. W. 1945. A limnological investigation of periphyton in Douglas Lake, Michigan. Trans. Am. Microsc. Soc. 64: 1-20.

Yvon-Durocher, G., J. I. Jones, M. Trimmer, G. Woodward, and J. M. Montoya. 2010. Warming alters the metabolic balance of ecosystems. Philos. Trans. R. Soc. London B Biol. Sci. 365: 2117-2126.

Yvon-Durocher, G., J. M. Montoya, M. Trimmer, and G. Woodward. 2011. Warming alters the size spectrum and shifts the distribution of biomass in freshwater ecosystems. Glob. Chang. Biol. 17: 1681-1694. 
Vander Zanden, M. J., and Y. Vadeboncoeur. 2002. Fishes as integrators of benthic and pelagic food webs in lakes. Ecology 83: 2152-2161.

Zárate, R., N. el Jaber-Vazdekis, N. Tejera, J. A. Pérez, and C. Rodríguez. 2017. Significance of long chain polyunsaturated fatty acids in human health. Clin. Transl. Med. 6: 25.

Zhao, Y., X. Xiong, C. Wu, Y. Xia, J. Li, and Y. Wu. 2018. Influence of light and temperature on the development and denitrification potential of periphytic biofilms. Sci. Total Environ. 613: $1430-1437$.

Zong-Ci, Z., L. Yong, and H. Jian-Bin. 2013. A review on evaluation methods of climate modeling. Adv. Clim. Chang. Res. 4: 137-144. 